UNIVERSITÉ LIBRE DE BRUXELLES

Faculté de Sciences

UNIVERSITÀ DEGLI STUDI DI TRIESTE

Dipartimento di Ingegneria e Architettura

\title{
Mathematical programming approaches to pricing problems
}

Alessia Violin
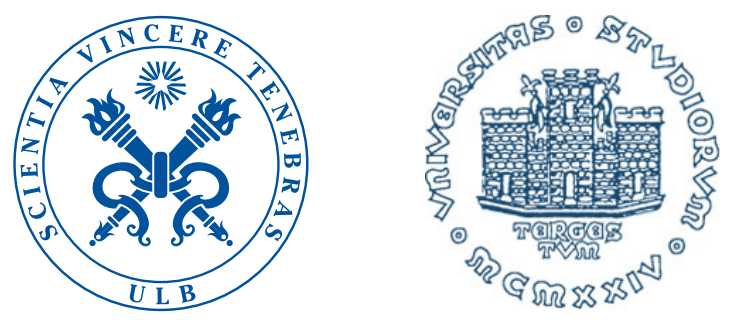

Thèse présentée en vue de l'obtention du grade académique de Docteur en Sciences

Tesi presentata per il conseguimento del titolo accademico di Dottore di Ricerca in Ingegneria dell'Informazione 
This thesis has been realised with the support from the Belgian national scientific funding agency "Fonds pour la Formation à la Recherche dans l'Industrie et dans l'Agriculture" (FRIA), of which the author was a research fellow between January 2011 and September 2014. This thesis has been composed by the author herself and contains original work of her own execution. Contributions of co-authors for some parts are acknowledged on page xxi

This thesis has been realised under the joint supervision of Prof. Martine Labbé (ULB) and Dr. Lorenzo Castelli (UniTs).

The members of the jury are:

- Dr. Lorenzo Castelli (Università degli Studi di Trieste, Italy)

- Prof. Bernard Fortz (Université Libre de Bruxelles, Belgium)

- Prof. Martine Labbé (Université Libre de Bruxelles, Belgium)

- Prof. Marco Lübbecke (RWTH Aachen University, Germany)

- Prof. Patrice Marcotte (Université de Montréal, Canada)

- Dr. Felice Andrea Pellegrino (Università degli Studi di Trieste, Italy)

- Prof. Jean-François Raskin (Université Libre de Bruxelles, Belgium)

- Prof. Thomas Stützle (Université Libre de Bruxelles, Belgium) 


\section{Abstract}

There are many real cases where a company needs to determine the price of its products so as to maximise its revenue or profit. To do so, the company must consider customers' reactions to these prices, as they may refuse to buy a given product or service if its price is too high. This is commonly known in literature as a pricing problem. This class of problems, which is typically bilevel, was first studied in the 1990s and is $\mathcal{N} \mathcal{P}$-hard, although polynomial algorithms do exist for some particular cases. Many questions are still open on this subject.

The aim of this thesis is to investigate mathematical properties of pricing problems, in order to find structural properties, formulations and solution methods that are as efficient as possible. In particular, we focus our attention on pricing problems over a network. In this framework, an authority owns a subset of arcs and imposes tolls on them, in an attempt to maximise his/her revenue, while users travel on the network, seeking for their minimum cost path.

First, we provide a detailed review of the state of the art on bilevel pricing problems. Then, we consider a particular case where the authority is using an unit toll scheme on his/her subset of arcs, imposing either the same toll on all of them, or a toll proportional to a given parameter particular to each arc (for instance a per kilometre toll). We show that if tolls are all equal then the complexity of the problem is polynomial, whereas in case of proportional tolls it is pseudo-polynomial. We then address a robust approach taking into account uncertainty on parameters. We solve some polynomial cases of the pricing problem where uncertainty is considered using an interval representation.

Finally, we focus on another particular case where toll arcs are connected such that they constitute a path, as occurs on highways. We develop a Dantzig-Wolfe reformulation and present a Branch-and-Cut-and-Price algorithm to solve it. Several improvements are proposed, both for the column generation algorithm used to solve the linear relaxation and for the branching part used to find integer solutions. Numerical results are also presented to highlight the efficiency of the proposed strategies. This problem is proved to be $\mathcal{A P X}$-hard and a theoretical comparison between our model and another one from the literature is carried out.

Keywords: Combinatorial optimisation, mixed-integer programming, bilevel programming, pricing, tolls, Dantzig-Wolfe, column generation, Branch-and-Price, SCIP 


\section{Résumé}

Un problème classique pour une compagnie est la tarification de ses produits à vendre sur le marché, de façon à maximiser les revenus. Dans ce contexte, il est important que la société prenne en compte le comportement de ses clients potentiels, puisque si le prix est trop élevé, ils peuvent décider de ne rien acheter. Ce problème est communément connu dans la littérature comme un problème de tarification ou "pricing". Une approche de programmation biniveau pour ce problème a été introduite dans les années 90, révélant sa difficulté. Cependant, certains cas particuliers peuvent être résolus par des algorithmes polynomiaux, et il y a encore de nombreuses questions ouvertes sur le sujet.

Cette thèse de doctorat porte sur les propriétés mathématiques des problèmes de tarification, fixant l'objectif de déterminer différentes formulations et méthodes de résolution les plus efficaces possibles, en se concentrant sur les problèmes appliqués aux réseaux de différents types. Dans les problèmes de tarification sur réseau, nous avons deux entités : une autorité qui possède un certain sous-ensemble d'arcs, et impose des péages, avec l'intention de maximiser les revenus provenant de celle-ci, et des utilisateurs qui choisissent leur chemin de moindre coût sur l'ensemble du réseau.

Dans la première partie de la thèse une analyse détaillée de l'état de l'art sur les problèmes de tarification biniveau est présentée, suivie, dans la deuxième partie, par une analyse de cas particuliers polynomiaux. En particulier, nous considérons le cas où l'autorité utilise un péage unitaire sur son sous-ensemble d'arcs, soit en choisissant le même péage sur chaque arc, soit en choisissant un péage proportionnel à un paramètre donné pour chaque arc (par exemple, un péage par kilomètre). Dans le premier cas de péages égaux, il est démontré que la complexité du problème est polynomiale, tandis que dans le second cas de péages proportionnels, elle est pseudo-polynomiale. Ensuite, nous présentons une première approche d'optimisation robuste pour les problèmes de tarification sur réseau, de manière à inclure de l'incertitude sur la valeur exacte des paramètres dans le modèle, qui est typique dans les problèmes réels. Cette incertitude est représentée en utilisant des intervalles pour les paramètres et nous proposons, pour certains cas, des algorithmes de résolution polynomiaux.

La troisième et dernière partie de la thèse concerne un cas difficile, le problème de tarification sur réseau dans lequel les arcs sont connectés de manière à constituer un chemin, comme c'est le cas pour les autoroutes. Initialement, nous prouvons que ce 
viii

problème est $\mathcal{A P} \mathcal{X}$-dur, renforçant le résultat connu jusqu'à maintenant. Ensuite, nous présentons des nouvelles formulations plus fortes, et en particulier, nous développons une reformulation de type Danztig-Wolfe, résolue par un algorithme de Branch-andCut-and-Price. Enfin, nous proposons différentes stratégies pour améliorer les performances de l'algorithme, pour ce qui concerne l'algorithme de génération de colonnes utilisé pour résoudre la relaxation linéaire, et pour ce qui concerne la résolution du problème avec variables binaires. Les résultats numériques complètent les résultats théoriques, en mettant en évidence l'efficacité des stratégies proposées.

Mots-clés: Optimisation combinatoire, programmation mixte entière, programmation biniveau, tarification, péages, Dantzig-Wolfe, génération de colonnes, Branch-and-Price, SCIP 


\section{Riassunto}

Un classico problema aziendale è la determinazione del prezzo dei prodotti da vendere sul mercato, in modo tale da massimizzare le entrate che ne deriveranno. In tale contesto è importante che l'azienda tenga in considerazione il comportamento dei propri potenziali clienti, in quanto questi ultimi potrebbero ritenere che il prezzo sia troppo alto e decidere dunque di non acquistare. Questo problema è comunemente noto in letteratura come problema di tariffazione o di "pricing". Tale problema è stato studiato negli anni novanta mediante un approccio bilivello, rivelandone l'alta complessità computazionale. Tuttavia alcuni casi particolari possono essere risolti mediante algoritmi polinomiali, e ci sono sono ancora molte domande aperte sull'argomento.

Questa tesi di dottorato si focalizza sulle proprietà matematiche dei problemi di tariffazione, ponendosi l'obiettivo di determinarne formulazioni e metodi risolutivi più efficienti possibili, concentrandosi sui problemi applicati a reti di vario tipo. Nei problemi di tariffazione su rete si hanno due entità: un'autorità che possiede un certo sottoinsieme di archi e vi impone dei pedaggi, con l'intento di massimizzare le entrate che ne derivano, e gli utenti che scelgono il proprio percorso a costo minimo sulla rete complessiva (a pedaggio e non).

Nella prima parte della tesi viene affrontata una dettagliata analisi dello stato dell'arte sui problemi di tariffazione bilivello, seguita, nella seconda parte, dall'analisi di particolari casi polinomiali del problema. In particolare si considera il caso in cui l'autorità utilizza uno schema di pedaggio unitario sul suo sottoinsieme di archi, imponendo o lo stesso pedaggio su ogni arco, o un pedaggio proporzionale a un dato parametro relativo ad ogni arco (ad esempio un pedaggio al chilometro). Nel primo caso di pedaggi uguali, si dimostra che la complessità del problema è polinomiale, mentre nel secondo caso di pedaggi proporzionali è pseudo-polinomiale. In seguito viene affrontato un approccio di ottimizzazione robusta per alcuni problemi di tariffazione su rete, in modo da includere nei modelli un'incertezza sul valore esatto dei parametri, tipica dei problemi reali. Tale incertezza viene rappresentata vincolando i parametri in degli intervalli e si propongono, per alcuni casi, algoritmi risolutivi polinomiali.

La terza e ultima parte della tesi riguarda un caso computazionalmente difficile, in cui gli archi tariffabili sono connessi in modo tale da costituire un cammino, come avviene per le autostrade. Inizialmente si dimostra che tale problema è $\mathcal{A} \mathcal{P} \mathcal{X}$-hard, rafforzando il risultato finora conosciuto. In seguito si considerano formulazioni più 
forti, e in particolare si sviluppa una riformulazione di Danztig-Wolfe, risolta tramite un algoritmo di Branch-and-Cut-and-Price. Infine si propongono diverse strategie per migliorare le performance dell'algoritmo, sia per quanto riguarda l'algoritmo di generazione di colonne utilizzato per risolvere il rilassamento lineare, sia per quanto riguarda la risoluzione del problema con variabili binarie. Risultati numerici complementano quelli teorici ed evidenziano l'efficacia delle strategie proposte.

Parole chiave: Ottimizzazione combinatoria, programmazione mista intera, programmazione bilivello, tariffazione, pedaggi, Dantzig-Wolfe, generazione di colonne, Branchand-Price, SCIP 


\section{Contents}

\begin{tabular}{lll}
\hline Abstract & v
\end{tabular}

Résumé vii

Riassunto ix

Contents $\quad$ xi

$\begin{array}{ll}\text { List of Tables } & \text { xv }\end{array}$

\begin{tabular}{ll}
\hline List of Figure & xvii
\end{tabular}

\begin{tabular}{|c|c|}
\hline List of Acronyms & xix \\
\hline
\end{tabular}

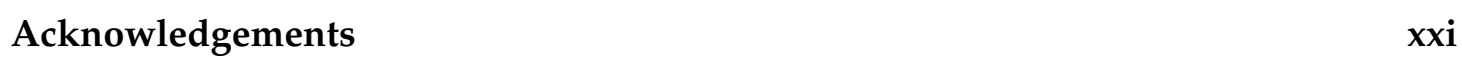

\begin{tabular}{ll}
\hline Introduction & 1
\end{tabular}

\begin{tabular}{lll}
\hline I STATE OF THE ART & 5
\end{tabular}

\begin{tabular}{|lll|l}
\hline & Bilevel Programming and Pricing Problems & 7
\end{tabular}

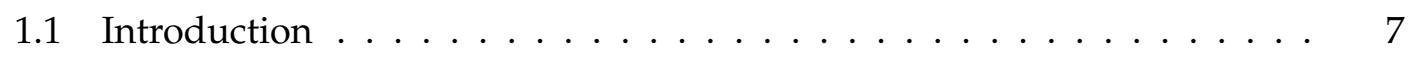

1.2 Bilevel Programming . . . . . . . . . . . . . . . . . . . . . . 7

1.3 The pricing problem $\ldots \ldots \ldots \ldots \ldots \ldots$

1.3 .1 The linear pricing problem . . . . . . . . . . . . . . . . . . . . . . . . . 14

1.4 The Network Pricing Problem . . . . . . . . . . . . . . . . . . . . . 15

1.4 .1 Arc pricing . . . . . . . . . . . . . . . . . . . . . 17

1.4 .2 The case of a single toll arc $\ldots \ldots \ldots \ldots 17$

1.4 .3 Complexity . . . . . . . . . . . . . . . . . . . 19

1.4 .4 One level MIP formulation . . . . . . . . . . . . . . 22

1.4 .5 Tight values for "big $\mathrm{M}$ and $\mathrm{N}^{\prime \prime} \ldots \ldots \ldots . \ldots . \ldots 23$

1.4 .6 The Shortest Path Graph Model . . . . . . . . . . . . . . . . . . . . . . . . . . . . . . . . 25

1.4 .7 Preprocessing . . . . . . . . . . . . . . . . 27 
$1.4 .8 \quad$ Numerical results . . . . . . . . . . . . . . . . . . . . . . . . . . . . 29

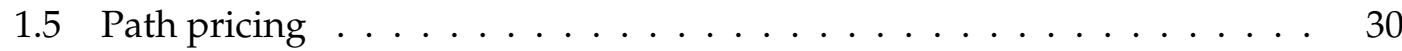

1.5 .1 Path pricing vs arc pricing . . . . . . . . . . . . . . . . . . . . . . . . . . . . . . . . . . . .

1.5 .2 Bilevel formulation . . . . . . . . . . . . . . . 32

1.5 .3 The case of a single commodity . . . . . . . . . . . . 33

1.5.4 The NPP with connected toll arcs or Highway Problem . . . . . . 33

1.5 .5 Approximability of the NPP with connected toll arcs . . . . . . 35

1.5 .6 One level non-linear formulation . . . . . . . . . . . . . . . 37

1.5 .7 One level MILP formulation. . . . . . . . . . . . . . . . . . . . . . . . . . . . . . . . . . . . . . .

1.5 .8 Valid inequalities . . . . . . . . . . . . . . . . . . . . 39

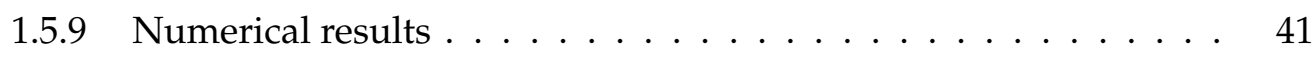

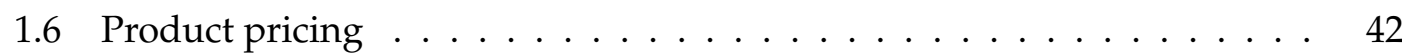

II SOME POLYNOMIAL PRICING PROBLEMS

2 Pricing Problems with Unit Toll 47

2.1 Introduction . . . . . . . . . . . . . . . . . . . . 47

2.2 Arc pricing and path pricing versions $\ldots \ldots \ldots \ldots$. . . . . . . . . . . . . . . .

$2.2 .1 \quad$ Arc pricing . . . . . . . . . . . . . . . . . 47

2.2 .2 Path pricing . . . . . . . . . . . . . . . . . . . . . . . . . . . . . . . . . 48

$2.2 .3 \quad$ Arc pricing vs path pricing . . . . . . . . . . . . . . . . . . . . . . 50

2.3 Properties and solution algorithm . . . . . . . . . . . . . . . . . . . . . 50

2.3 .1 Path dominance criterion . . . . . . . . . . . . . . . . 50

2.3 .2 Sequential solution procedure $\ldots \ldots \ldots \ldots \ldots$

2.3 .3 (Pseudo-)polynomial path formulation . . . . . . . . . . . . . . . . . . 54

2.4 Relation with the parametric shortest path problem . . . . . . . . . . . 55

2.4 .1 The parametric shortest path problem . . . . . . . . . . . . 55

2.4 .2 Relation between unit toll problems and the parametric shortest

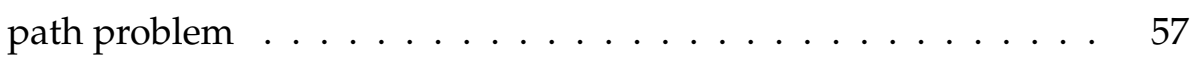

3 Robust Version of Some Polynomial Pricing Problems 59

3.1 Introduction . . . . . . . . . . . . . . . . . . . . . . 59

3.2 A brief description of robustness . . . . . . . . . . . . . . 60

3.3 The case of a single toll arc and multiple commodities . . . . . . . . . 62

3.3.1 Worst case maximum revenue with interval demand . . . . . . . 63

3.3.2 Worst case maximum revenue with interval M parameters . . . . 65

3.4 The case of a single commodity and multiple toll arcs . . . . . . . . . . 69

3.4 .1 Worst case maximum revenue with interval demand . . . . . . . 70

3.4 .2 Worst case maximum revenue with interval M parameters . . . . 71

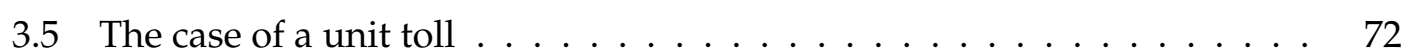

3.5 .1 Worst case maximum revenue with interval demand . . . . . . 73 
3.5.2 Worst case maximum revenue with interval M parameters . . . . 74

$\begin{array}{ll}\text { III AN NP-HARD PRICING PROBLEM } & 75\end{array}$

$4 \quad$ A Dantzig-Wolfe Reformulation for the NPP with Connected Toll Arcs $\quad 77$

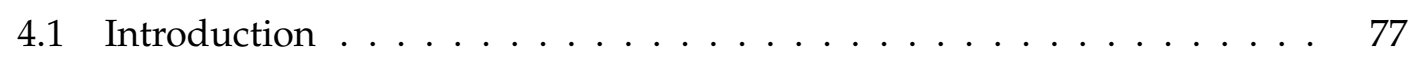

4.2 Dantzig-Wolfe reformulation of an Integer Problem . . . . . . . . . . . 78

$4.3 \quad$ A Dantzig-Wolfe reformulation for the NPP with connected toll arcs . . 80

$4.3 .1 \quad$ Master Problem . . . . . . . . . . . . . . . . . . 80

4.3 .2 Subproblem . . . . . . . . . . . . . . . . 83

4.3 .3 Dual bound for column generation . . . . . . . . . . . . . . . 85

$4.3 .4 \quad$ Description of instances for numerical experiments . . . . . . . 85

4.3 .5 Numerical results . . . . . . . . . . . . . . . . . . . . . 87

4.4 An extended formulation . . . . . . . . . . . . . . . . . . . . . . . . . . . 89

4.5 Comparison between the formulations . . . . . . . . . . . . . . . . 93

4.5 .1 Theoretical comparison . . . . . . . . . . . . . . . . 93

4.5 .2 Numerical results . . . . . . . . . . . . . . . . . . . . . 100

4.6 Initialisation of the (MP), infeasibility and Farkas pricing . . . . . . . . 102

4.6 .1 Initialisation . . . . . . . . . . . . . . . . . . 103

4.6 .2 Infeasibility and Farkas pricing . . . . . . . . . . . . . 107

4.7 Adding more columns at each iteration . . . . . . . . . . . . . . 108

4.8 Additional stopping criterion using dual bound . . . . . . . . . . . . . . 112

4.9 Stabilisation procedure for solving the (MP) with column generation . . 113

$4.9 .1 \quad$ Boxtep method with slack variables . . . . . . . . . . . . . 113

4.9 .2 Dual variable smoothing approach . . . . . . . . . . . . . . . . . . . . . . . . . . . . . . . . . . . .

4.9 .3 Numerical results . . . . . . . . . . . . . . . . . . . . . . . . . . . . . 119

4.10 Best configuration of the column generation algorithm for the linear relaxation of the NPP with connected toll arcs . . . . . . . . . . . . . . . 126

4.10 .1 List of parameters to define the column generation algorithm . . 126

4.10 .2 Automatic tuning of the parameters . . . . . . . . . . . . 127

4.10 .3 Numerical results . . . . . . . . . . . . . . . . . . . . . . . . . 132

4.11 Valid inequalities . . . . . . . . . . . . . . . . . . 136

4.11 .1 Modified master problem . . . . . . . . . . . . . . . . . . . . . . . . . . . . . . . . . . . . . . .

4.11 .2 Modified subproblem . . . . . . . . . . . . . . . . 137

4.11 .3 Modified dual bound for column generation . . . . . . . . . . . 138

4.11 .4 Numerical results . . . . . . . . . . . . . . . . . . . . . . . . 138

5 Branch-(and-Cut)-and-Price framework for the NPP with Connected Toll Arcs 145

5.1 Introduction . . . . . . . . . . . . . . . . . . . . . 145

5.2 The price setting subproblem . . . . . . . . . . . . . . . . . 145

5.3 The Inverse Problem for the NPP with connected toll arcs . . . . . . . . 150 
$5.4 \quad$ Heuristics for the NPP with connected toll arcs . . . . . . . . . . . . . . . 153

5.4 .1 Heuristic 1 . . . . . . . . . . . . . . . . . . . . . . . . . . 154

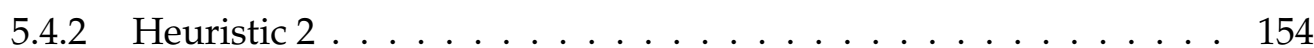

5.5 Branching rules . . . . . . . . . . . . . . . . . . . . . . . 155

5.5 .1 Enforcing branching decisions in the subproblem . . . . . . . 156

5.5 .2 Explicit vs implicit branching . . . . . . . . . . . . . . . . . 157

5.5 .3 Implicit branching on constraints . . . . . . . . . . . . . . . . . 159

5.6 Additional stopping criterion using dual bound for the integer problem 165

5.7 Integrating heuristic solutions in the Branch-and-Price . . . . . . . . . 166

5.8 Branch-and-Cut-and-Price framework . . . . . . . . . . . . . . . . . . 167

5.8 .1 List of parameters to define the HP-B\&C\&P algorithm . . . . . . 167

5.8 .2 Automatic tuning of the parameters . . . . . . . . . . . . . . 169

5.8 .3 Numerical results . . . . . . . . . . . . . . . . . . . . . . . . 172

\begin{tabular}{ll}
\hline Conclusion & 179
\end{tabular}

\begin{tabular}{ll}
\hline Bibliography & 183
\end{tabular} 


\section{List of Tables}

1.1 Stackelberg vs Nash example: payoff matrix . . . . . . . . . . . . . . 9

\begin{tabular}{|ll|l|l|l|}
\hline 1.2 & Feasible values for $M_{a}$ for the four networks in Figure & 1.8 & (dominant \\
\hline
\end{tabular} values in bold $] \ldots \ldots \ldots \ldots . \ldots \ldots \ldots$

1.3 Numerical results for arc pricing (from Dewez et al. 2008) $\ldots . . . . \quad 30$

$1.4 \quad$ Numerical results for path pricing (from Heilporn et al., 2011). . . . . . 42

$4.1 \quad$ Numerical results on the column generation algorithm for the linear

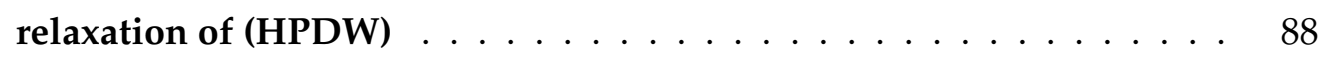

4.2 Numerical results on linear relaxation for (HPL), (HPDW) and (HPEF) formulations . . . . . . . . . . . . . . . . . . . . . . . 101

4.3 Performance ratio of (HPDW-LR) formulation with respect to (HPLLR) formulation . . . . . . . . . . . . . . . . . . . . . 102

4.4 Numerical results on different initialisations for the linear relaxation of (HPDW) $\ldots \ldots \ldots$. . . . . . . . . . . . . . . . . . 105

4.5 Numerical results on the number of columns added at each iteration for the linear relaxation of (HPDW) - Complete and partial graph in-

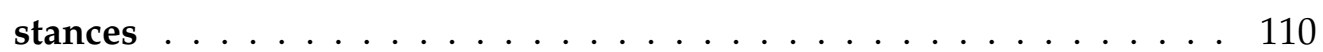

4.6 Numerical results on the number of columns added at each iteration for the linear relaxation of (HPDW) - Shioda et al. and A1 instances . . 111

4.7 Numerical results on different stabilisation strategies for (HPDW-LR) (best results in bold) - Complete graph instances . . . . . . . . . . . . 121

4.8 Numerical results on different stabilisation strategies for (HPDW-LR) (best results in bold) - Partial graph instances . . . . . . . . . . . 122

4.9 Numerical results on different stabilisation strategies for (HPDW-LR) (best results in bold) - Shioda et al. instances . . . . . . . . . . . . . 123

4.10 Numerical results on different stabilisation strategies for (HPDW-LR) (best results in bold) - A1 instances . . . . . . . . . . . . . . . . . 124

4.11 Best configurations of parameters for each instances type - irace results 128

4.12 Best configurations of parameters for all instances types - irace results 129

4.13 Numerical results on the best configuration of the column generation algorithm for the linear relaxation of (HPDW) - Complete and partial graph instances . . . . . . . . . . . . . . . . . . 132 
4.14 Numerical results on the best configuration of the column generation algorithm for the linear relaxation of (HPDW) - Shioda et al. and A1 instances . . . . . . . . . . . . . . . . . . . . . 133

4.15 Performance ratio of the basic and the best configurations of the column generation algorithm for (HPDW-LR) formulation with respect to (HPL-LR) formulation . . . . . . . . . . . . . . . . . . . . . . 135

4.16 Numerical results on adding SSPI to the column generation algorithm for the linear relaxation of (HPDW) - Complete and partial graph instances . . . . . . . . . . . . . . . . . . . . . . . . . . . 139

4.17 Numerical results on adding SSPI to the column generation algorithm for the linear relaxation of (HPDW) - Shioda et al. and A1 instances . . 140

4.18 Numerical results on a different separation strategy when adding SSPI to the column generation algorithm for the linear relaxation of (HPDW) 141

4.19 Numerical results on linear relaxation for (HPL) and (HPDW) formulations with SSPI - Complete and partial graph instances . . . . . . . . 143

4.20 Numerical results on linear relaxation for (HPL) and (HPDW) formulations with SSPI - Shioda et al. and A1 instances. . . . . . . . . . . . . 144

$5.1 \quad$ Numerical results on the impact of explicit original $x_{a}^{k}$ variables when solving the linear relaxation of (HPDW) $\ldots \ldots$. . . . . . . . . . . . . . . 159

5.2 Best configurations of parameters for each instances set - irace results 171

5.3 Numerical results on solving (HP) for complete graph instances, comparing (HPL) and (HPDW) with different configurations of HP-B\&C\&P 177

5.4 Numerical results on solving (HP) for A1 instances, comparing (HPL) and (HPDW) with different configurations of HP-B\&C\&P $\ldots . . . .178$ 


\section{List of Figures}

1.1 Graphical example of the objective functions of the (bi)linear pricing problem in a two-dimensional case (Labbé et al. [1998) . . . . . . . . . . 15

1.2 Example of a NPP (from Dewez, 2004) . . . . . . . . . . . . . 16

1.3 Example of a NPP with negative tolls (from Labbé et al., 1998] . . . . . 16

\begin{tabular}{|lll}
1.4 & Leader revenue (in bold) in the case of a single toll arc pricing problem 18
\end{tabular}

1.5 Strong $\mathcal{N} \mathcal{P}$-hard proof for the NPP: subnetwork for one clause $\left(l_{i, 1} \vee\right.$

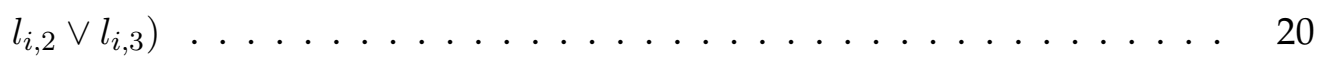

1.6 Strong $\mathcal{N} \mathcal{P}$-hard proof for the NPP: network for the formula $\left(x_{1} \vee x_{2} \vee\right.$ $\left.\overline{x_{3}}\right) \wedge\left(\overline{x_{2}} \vee x_{3} \vee \overline{x_{4}}\right) \wedge\left(\overline{x_{1}} \vee x_{3} \vee x_{4}\right)($ from Roch et al., 2005) . . . . . . 21

\begin{tabular}{|lll}
1.7 & Paths that occur in the calculation of valid values for "big $\mathbf{M}^{\prime \prime}$
\end{tabular}$\ldots$. . . . 24

1.8 Example of networks for the calculation of valid values on "big $\mathbf{M}^{\text {" }}$ ((b), (c) and (d) from Dewez, 2004) . . . . . . . . . . . . . . 25

1.9 Example of the structure of different possible paths in a network with

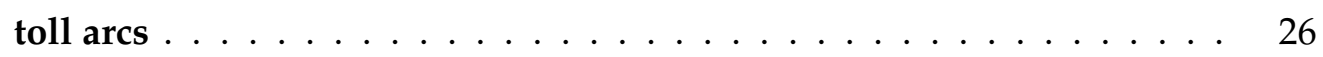

1.10 Example of Shortest Path Graph reduction (from Dewez, 2004)] . . . . 26

1.11 Example of arc reduction (from Bouhtou et al. 2007b) . . . . . . . . 28

1.12 Example of dominated paths reduction . . . . . . . . . . . 28

1.13 Network with an exponential number of undominated paths. . . . . . 29

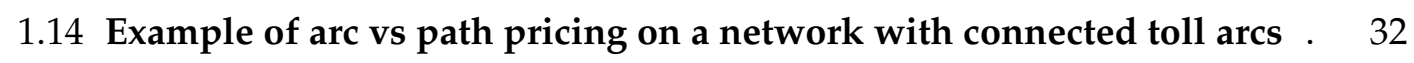

1.15 Complete toll NPP (from Heilporn et al. 2010b)] . . . . . . . . . . . 34

1.16 Subnetworks on which triangle and monotonicity inequalities apply (from Heilporn et al. [2010b] . . . . . . . . . . . . . 35

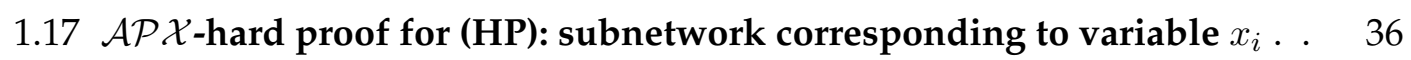

$1.18 \mathcal{A P} \mathcal{X}$-hard proof for (HP): subnetwork for clause-commodities $\left(x_{j} \vee\right.$

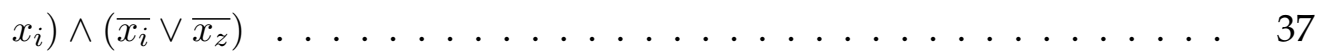

2.1 Path dominance criterion for PT-NPP . . . . . . . . . . . . . . 51

$\begin{array}{lll}2.2 & \text { Sequential solution procedure for PT-NPP - Functions on } T & \ldots\end{array} \quad 53$

$3.1 \quad$ Examples: representation of $M^{k}$ intervals on the toll axis . . . . . . 68

$3.2 \quad$ Partitions of the commodities set $\mathcal{K}$ for $T_{1}$ and $T_{2} \ldots \ldots \ldots \ldots$ 
4.1 Primal and dual bound evolution while solving the linear relaxation of (HPDW) with column generation . . . . . . . . . . . . . . . . 89

$4.2 \quad$ Representation of $T_{a}$ in the interval of two subsequent $m_{a}^{l}$ values . . . 89

4.3 Example of a network with a better bound from (HPDW-LR) with respect to (HPL-LR) . . . . . . . . . . . . . . . . . 94

4.4 Performance profile graphs on the gap for the linear relaxation for (HPL), (HPDW) and (HPEF) . . . . . . . . . . . . . . . . . . 103

4.5 Performance profile graphs on the solution time for the linear relaxation of (HPDW) and (HPEF) . . . . . . . . . . . . . . . . . 104

\begin{tabular}{|lll}
4.6 & Performance profile graphs on different initialisations for (HPDW-LR) 106
\end{tabular}

4.7 Primal and dual bounds evolution while solving (HPDW) with column generation - Different initialisations performance $\ldots \ldots$. . . . 107

4.8 Performance profile graphs on adding more columns at each iteration for (HPDW-LR) . . . . . . . . . . . . . . . . . . . . 109

4.9 Primal and dual bounds evolution while solving (HPDW) with column generation - Adding more columns per iteration performance . . 112

4.10 Performance profile graphs on different stabilisation strategies for (HPDW-

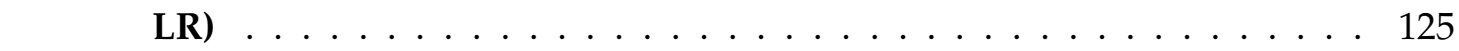

4.11 Primal and dual bounds evolution while solving (HPDW) with column generation - Different stabilisation strategies performance . . . . 125

4.12 Performance profile graphs on the best configurations of the column generation algorithm found by irace for each instance type .

4.13 Performance profile graphs on the best configurations of the column generation algorithm found by irace with the overall tuning (compared with the best configuration found in the separate tuning) . . . . 130

4.14 Performance profile graphs on the best column generation algorithm for (HPDW-LR)

4.15 Primal and dual bounds evolution while solving the linear relaxation of (HPDW) with the best configuration of the column generation . . . 135

4.16 Performance profile graphs on the gap for the linear relaxation for (HPL) and (HPDW) with SSPI . . . . . . . . . . . . . . 142

5.1 Flow network for (D-PSS) formulation . . . . . . . . . . . . . . . . . 149

5.2 Branching on original variable $x_{a}^{k} \ldots \ldots \ldots \ldots$. . . . . . . . . 161

5.3 Tree for standard branching on SOS variables . . . . . . . . . . . . . . 164

5.4 SOS branching rule $\ldots \ldots \ldots \ldots$. . . . . . . . . . . . . . . . . . . 164

5.5 Performance profile graphs on solving (HP) for complete graph instances, comparing (HPL) and (HPDW) with different configurations of HP-B\&C\&P.

5.6 Performance profile graphs on solving (HP) for A1 instances, comparing (HPL) and (HPDW) with different configurations of HP-B\&C\&P . 175 


\section{List of Acronyms}

(alphabetical order)

\begin{tabular}{|c|c|c|}
\hline BP & Bilevel Problem & Section 1.2 page 7 \\
\hline CT-NPP & Constant Toll Network Pricing Problem & Section 2.1. page 47 \\
\hline D-IP & Dual of Inverse Problem (of HP) & Section 5.3, page 150 \\
\hline D-PSS & Dual of Price Setting Subproblem & Section 5.2 , page \\
\hline DW & Dantzig-Wolfe & Section 4.2 page \\
\hline H1 & Heuristic 1 (for HP) & Section 5.4 .1 , page 154 \\
\hline H2 & Heuristic 2 (for HP) & Section 5.4 .2 page 154 \\
\hline HP & Highway Problem & Section 1.5 .4 , page 33 \\
\hline HP-B\&C\&P & HP Branch-and-Cut-and-Price (algorithm) & Section 5.8, page 167 \\
\hline HPDW & Highway Problem Dantzig Wolfe (reformulation) & Section 4.3 , page \\
\hline HPEF & Highway Problem Extended Formulation & Section 4.4 page \\
\hline HPL & Highway Problem Linear (formulation) & Section 1.5.7. pa \\
\hline HPNL & Highway Problem Non Linear (formulation) & Section 1.5 .6 , page 37 \\
\hline IP & Inverse Problem (of HP) & Section 5.3 , page 150 \\
\hline MP & Master Problem (for a general DW reformulation) & Section 4.2 , page \\
\hline MP & Master Problem (for the HPDW reformulation) & Section $4.3 .1 . \mathrm{pa}$ \\
\hline MP-EXP & MP Explicit (for the HPDW reformulation) & Section 5.5.2, page 157 \\
\hline NPP & Network Pricing Problem & Section 1.4 , page 15 \\
\hline PSPP & Parametric Shortest Path Problem & Section 2.4.1 pag \\
\hline PSS & Price Setting Subproblem & Section 5.2, page \\
\hline PT-NPP & Proportional Toll Network Pricing Problem & Section 2.1, page \\
\hline SOS & Special Ordered Set (branching rule) & Section 5.5.3, page \\
\hline SP & Sub Problem (for a general DW reformulation) & Section 4.2 , page \\
\hline SP & Sub Problem (for the HPDW reformulation) & Section 4.3.2, page 83 \\
\hline SPGM & Shortest Path Graph Model & Section 1.4.6, pag \\
\hline SSPI & Strengthened Shortest Path Inequalities & Section 1.5 .8 , page 39 \\
\hline
\end{tabular}




\section{Acknowledgements}

First of all I would like to thank both of my supervisors, Martine Labbé and Lorenzo Castelli. Without their support and advice I would not have been able to carry out my Ph.D. Their contributions to my advancement have been complementary.

Martine provided me with invaluable advice on the general direction of my research, as well as helping me to get to grips with the most mathematical aspects. By encouraging me to always read and study more, she pushed me to greatly improve my operational research "culture". Her patience in correcting each of the articles I wrote several times and in great detail, as well as this Ph.D manuscript, taught me the importance of being precise and rigorous in my writing. Finally I would like to thank her for pushing me to be more independent and always to first try to solve the problems I encounter on my path by myself, even if I admit it was sometimes a source of frustration; this has allowed me to feel more proud of the results that I have now achieved.

I have to thank Lorenzo first of all for introducing me to the world of academic research through my Master's thesis; if I had not enjoyed it so much I would not have continued with a Ph.D. During these last four years he has given me his constant support, always being available to discuss ideas, and providing a positive outlook when results did not go my way and when my progress seemed to be blocked. He also took the time to verify in great detail the algorithms I proposed in the second part of my thesis and provided useful comments on how to improve them, in addition to giving me advice on how to make this Ph.D manuscript clearer and easier to read.

I am also very grateful to Bernard Fortz, who co-authored the third part of my thesis. His help and advice in the development of the models and of the Branch-and-Price algorithm was fundamental, and more generally he was always available to discuss and clarify my doubts, even though he was not my direct supervisor.

I thank Leslie Pérez Cáceres from the IRIDIA research group who carried out the tuning of the column generation and Branch-and-Price algorithms presented in the third part of this thesis, and her supervisor Thomas Stüzle for the suggestions on the development of these algorithms. Leslie has been very patient in repeating the tuning many times and when we encountered various implementation-related issues. She always found time even when she was very busy, and I enjoyed collaborating with her.

I would like to thank all the other members of my Ph.D jury for their precious time reading my thesis and for their constructive comments: Prof. Marco Lübbecke (RWTH 
xxii

Aachen University), Prof. Patrice Marcotte (Université de Montréal), Dr. Felice Andrea Pellegrino (Università degli Studi di Trieste) and Prof. Jean-François Raskin (ULB).

A warm thank you goes to Tue Christensen who helped me to start using SCIP, and took the time to show me how it works and how to debug it. Without his help it would surely have taken me much longer to complete this work, and knowing that he was there to answer my questions was of great support at the beginning of my "implementation adventure". A sincere thanks also goes to all the SCIP team, who, with their mailing list, provide an invaluable help to SCIP users, and who promptly answered all of my questions, no matter how basic or challenging. In particular I would like to thank Gerald Gamrath for giving me the opportunity to visit their group in ZIB, Berlin, and who gave me some very useful suggestions to improve my implementation by fully using SCIP's capabilities.

I would like to thank all (former and actual) members of my research group, the "Graphes et Optimisation Mathématique (GOM)", for always being there to discuss science but also, and probably most importantly, for a coffee break or a small chat to recharge batteries. They provided with a very pleasant working environment, which I believe played an important part in my achievements, and some of them are now very good friends. I also thank all the computer science department lunch group, always coming up with hilarious topics for our lunch breaks, and for all the fun evenings we have had together.

A big thanks goes to all my very numerous ex-flatemates, who, from the start of my stay in Brussels, always made me feeling at home, as well as to Eurogirls, for the great team-spirit during the volleyball matches and outside the gym, and to all of the good friends who made me enjoy my time in Brussels so much, with great parties, dinners, trips, and any sort of activity. A special thanks goes to Ivana, our evening chats are always a great support, and to Paola, for the great "experienced" advice.

Un affettuoso ringraziamento va ad Alessia, amica di sempre, riesci sempre a farmi sentire come se non fossi mai partita, e a tutti gli amici di Trieste che si ricordano sempre di me nonostante la distanza.

Un sentito grazie va alla mia famiglia, a mamma e papà, per tutto quello che mi hanno insegnato e che $\mathrm{mi}$ ha portato a raggiungere questo importante traguardo, per essere sempre presenti senza imporre le proprie idee e per accettare le mie scelte.

And finally, the biggest thank you goes to Christopher for simply always being there, and for having kept up my motivation during good times and bad. 




\section{Introduction}

In many real cases, a company is confronted with a best price strategy problem: it wishes to determine the price of a set of products in order to maximise its revenue. The reaction of potential customers has to be taken into account: if prices are too high, they may decide not to buy the products, whereas if prices are too low, the company looses revenue. Pricing problems have been widely present in the economic literature for many decades. Furthermore, over the past two decades, this type of problem has attracted increasing attention from researchers in the operational research community, interested in using it to model a range of different applications, from product pricing to highway systems and telecommunication services. Many results have been obtained, but as the problem is very challenging there is still a whole range of interesting and open questions to be addressed, both from structural and algorithmic points of view.

The pricing problem can be seen as a two-level game between a company and its customers. First the company sets the prices for a set of goods or services, with the aim of maximising its revenue, and then customers react and decide if they are willing to pay these prices. This is a Stackelberg game framework in which the company is the leader and customers are the followers. Setting the price at a level that maximises the company's revenue requires considerable market knowledge and algorithmic analysis, in order to know customers' preferences and to be able to model their reactions.

In a mathematical programming approach the relationship between the choices of the company and the purchase decision of its customers can be represented as a Bilevel optimisation Problem (BP). This framework consists of a hierarchical structure with a first level optimisation problem, which contains certain constraints specifying that the solution must be at the same time optimal to another (second level) optimisation problem. The values of the decision variables of the first problem influence the optimal solution of the second problem and vice versa.

In particular, in a bilevel price setting problem the leader (first level) sets some taxes or prices for a set of activities, and the followers (second level) select activities from amongst taxed and untaxed ones with the aim of minimising their operating costs. Applying these sequential pricing problems to a network, we obtain the Network Pricing Problem (NPP), in which an authority owns a subset of arcs and imposes tolls on them, and users travel on the network. The authority is the leader that wants to maximise his/her revenue, and network users are the followers who want to minimise their costs, 
and so will always travel on their minimum total cost path, paying fixed costs (fuel, time, ...) plus tolls.

This thesis focuses on a mathematical programming approach for pricing problems, with the aim of identifying structural properties and solution methods that are as efficient as possible. These problems are intrinsically difficult, so attention focuses on underlining particular aspects and cases to study in depth, investigating their complexity, developing (if possible) linear mixed-integer bilevel and single level formulations, and ad hoc solution methods. Theoretical results are complemented with numerical results on different sets of instances, to analyse the efficiency of the proposed algorithms.

The thesis is divided into three main parts: first a review of the state of the art, second a description of results on "easy" problems and third an attempt to tackle a "difficult" problem.

The first part, presented in Chapter 1, consists of a detailed review of the state of the art of the subject. We present pricing problems modelled as bilevel programs, with special attention to network applications, providing different formulations, complexity results, properties and numerical results. This review was the starting point for the research presented in this thesis and allowed us to identify interesting aspects for investigation. Furthermore, when analysing a particular case of the NPP where toll arcs are connected, we were able to strengthen its complexity result and the $\mathcal{A P} \mathcal{X}$-hardness proof is presented in this first part of the manuscript.

The second part of the thesis, composed of Chapters 2 and 3 , presents results of easy (polynomial or pseudo-polynomial) versions of the NPP. In Chapter 2, we introduce two new particular cases of the NPP: in the first one the authority imposes the same toll on all his/her toll arcs, whilst in the second one the imposed toll is proportional to a given parameter associated with each arc (e.g. the arc length). In both cases the authority is restricted to a unique toll scheme, which makes the problem simpler. We prove that the first case is polynomial whilst the second one is pseudo-polynomial. We present different formulations for these problems and show that a solving procedure similar to the one proposed in the literature for the single toll arc case can be used. Furthermore, we show that there is a relation between them and the parametric shortest path problem. We compile results from the literature for the parametric shortest path problem and show that they can be used to solve both cases of the unit toll NPP. Chapter 3 considers some easy problems such as the single toll arc case, the single commodity case and the unit toll case of the NPP and introduces uncertainty on some of their parameters. A robust approach where unknown parameters are constrained to lie in known intervals is considered, and solutions are sought for the worst case scenario. We first provide a brief introduction to robust optimisation with interval representation for uncertainty. We then analyse each problem with uncertain demand and cost parameters, and show how solving algorithms can be modified to tackle each situation. In particular, we notice that if the demand or the toll free path cost are uncertain, the single commodity case solution is not modified, whilst the single toll arc case and the 
unit toll case solutions are modified, but their solving algorithm remains polynomial (pseudo-polynomial for the unit toll case with proportional toll).

The third part of the thesis, presented in Chapters 4 and 5, addresses an $\mathcal{N} \mathcal{P}$-hard case of the NPP, where toll arcs are connected such that they constitute a path. We develop a Dantzig-Wolfe reformulation for the problem and prove that its linear relaxation is at least as strong as the linear relaxation of the mixed-integer formulation presented in the literature. Further, we show that for many instances it is strictly better with a significant improvement. We also present an extended mixed-integer formulation having the same linear relaxation value as that of the Dantzig-Wolfe reformulation. A column generation algorithm is proposed to solve the linear relaxation of the Dantzig-Wolfe reformulation, and several strategies are adopted to improve the performance of the algorithm, such as different initialisation and stabilisation approaches amongst others. Further, branching strategies are considered to construct the tree in a Branch-and-Price framework. Valid inequalities from the literature are also analysed and reformulated in order to be included in our algorithm. Numerical results, obtained using the SCIP framework, assess the efficiency of each strategy and of our Branch-andCut-and-Price algorithm. Chapter 4 presents the reformulation and the linear relaxation results, whilst Chapter 5 focuses on the branching and the complete Branch-(and-Cut)and-Price algorithm. Moreover, also in Chapter 5, this approach is complemented by studying the problem of finding good integer solutions and proposing heuristic algorithms that can be integrated in the tree search. The best configurations of the column generation and the Branch-and-Cut-and-Price algorithms have been chosen using irace, a framework for the automatic tuning of parameters of optimisation algorithms.

Finally the last chapter summarises the main contributions of this thesis and presents remaining open questions, as well as some more general ideas for future work. 
Part I

State of the art 



\section{Chapter 1}

\section{Bilevel Programming and Pricing Problems}

\subsection{Introduction}

In this chapter, we introduce the problems that are object of study of this thesis, providing a detailed state of the art of the literature. We present how pricing problems can be modelled using a bilevel programming framework. We review the general problem as well as particular cases that have been studied, reporting different formulations, properties and complexity results.

This chapter is organised as follows. In Section 1.2, we briefly present the general Bilevel Programming framework, and then pricing problems are introduced in Section 1.3. as well as some mathematical models and properties for these schemes. As these subjects are not the central topic of this thesis research, we only provide a short introduction to them, necessary for understanding the remainder of the manuscript. Next, we focus on pricing problems based on networks (Section 1.4). We present in detail models, solution methods and various properties developed in the literature as well as some numerical results to confirm their efficiency. In Section 1.5, we describe a variant of the problem involving pricing on paths instead of arcs of the network. The $\mathcal{A P X}$ hardness proof that we developed for this path pricing version is also presented in this section. Finally, a further application to product pricing is described in Section 1.6

\subsection{Bilevel Programming}

Several real-world problems involve a hierarchical relationship between two decision levels, in areas like management (facility location, environmental regulation, credit allocation, energy policy, hazardous material regulation), economic planning (social and agricultural policies, electric power pricing, oil production), or engineering (optimal design, structures and shape). In real-world sequential models therefore, the choice of a decision-maker, i.e. the upper level, leads to some reaction within a particular market 
or social entity, which corresponds to the lower level of the problem.

In mathematical programming a Bilevel Programming (BP) problem is a hierarchical optimisation problem in which part of the constraints translates the fact that some of the variables constitute an optimal solution to a second optimisation problem. These problems were introduced by Bracken and McGill (1973) as mathematical programs with optimisation problems in the constraints, whereas the terms bilevel and multilevel were later introduced by Candler and Norton (1977).

In this setting the first objective function and its proper constraints, which are not related to the second optimisation problem, usually refer to the so-called leader or first level, whilst the second optimisation problem (objective function and constraints) refers to the follower or second level. This terminology reflects the sequentiality of the problem: the follower chooses his/her optimal solution once the leader's choice is known, and the leader will therefore optimise his/her choice taking into account that the follower always reacts optimally to it.

For given values of the first level decision variables, the second level problem may have multiple optimal solutions. In this case, different modelling approaches can be proposed depending on the follower's behaviour. A cooperative behaviour leads to an optimistic solution, such that when there are multiple solutions the leader assumes that the follower's choice is always the one most favourable to him/her. On the contrary, an aggressive behaviour leads to a pessimistic solution, where the leader protects himself against the follower's worst possible reaction (Colson et al., 2007). A more complete discussions of these issues can be found in Loridan and Morgan (1996) and Dempe (2002). In this thesis, we only consider cooperative behaviour.

Let $x$ and $y$ denote decision vectors, $f$ and $g$ objective functions, and $X$ and $Y$ the feasible solution sets of the leader and the follower respectively. The general BP problem can be formulated as:

$$
\begin{array}{cl}
\max _{x, y} & f(x, y), \\
\text { s.t. } & (x, y) \in X, \\
& y \in S(x), \\
\text { where } & S(x)=\arg \min _{y} g(x, y), \\
& \text { s.t. }(x, y) \in Y .
\end{array}
$$

In game theory, the BP problem has been introduced under the name of Stackelberg game (Stackelberg, 1952). In such a game there are two players, a leader $L$ and a follower $F$. The leader plays first and decides his/her best strategy, taking into account that the follower reacts in an optimal way to his/her choice. Player $F$ plays second, and so already knows $L$ 's choice of strategy when choosing his/her own.

We present an example in Table 1.1. with two players, called 1 and 2. They both have two strategies, denoted by A and B, and they want to maximise their own gain. 
Gains for all players and strategies, also often called payoffs, are reported in the table. If Player 1 is the leader and Player 2 is the follower, the Stackelberg solution consists of strategy B for both players, leading to a gain of 3 for Player 1 and of 1 for Player 2. On the contrary, if Player 2 is the leader and Player 1 the follower, the Stackelberg solution consists of strategy A for both players, leading to a gain of 2 for both of them.

\begin{tabular}{cc|cc} 
& & \multicolumn{2}{|c}{ Player 2 } \\
& & Strategy A & Strategy B \\
\hline \multirow{2}{*}{ Player 1 } & Strategy A & 2,2 & 4,1 \\
& Strategy B & 1,0 & 3,1
\end{tabular}

Table 1.1: Stackelberg vs Nash example: payoff matrix

Another well known solution concept in game theory is the Nash equilibrium (see e.g. Owen. 1968), which is appropriate for a simultaneous game (i.e. players play at the same time, without any hierarchical structure) or games that are repeated many times. Specifically, a Nash equilibrium consists in a solution in which no player has an interest in changing his/her choice of strategy, as it cannot lead to a better gain. For the game described by the payoff matrix in Table 1.1, the Nash equilibrium is unique and given by strategy A for both players, leading to a gain of 2 for both of them. It is therefore interesting to underline the conceptual difference between a Stackelberg solution and a Nash equilibrium: they correspond to different assumptions in the game rules, as the former is sequential with a precise hierarchical structure. They generally lead to different solutions. One can also point out that, under mild conditions, there is always a Stackelberg solution. On the contrary, a Nash equilibrium may not exist, as it could occur that, for all possible pairs of strategies, at least one player would have an interest in changing his/her choice.

From a computational point of view, bilevel problems are intrinsically difficult. Even the simple version of a BP problem where the objective functions and the constraints are linear has been shown to be $\mathcal{N} \mathcal{P}$-hard by Jeroslow (1985). Furthermore, Hansen et al. (1992) prove the strong $\mathcal{N} \mathcal{P}$-hardness of the problem. Vicente et al. (1994) strengthen these results and prove that merely checking strict local optimality and checking local optimality in linear BP problems are $\mathcal{N} \mathcal{P}$-hard problems.

Due to the difficulty of BP, solution methods and algorithms generally focus on particular cases where functions have convenient properties, such as linearity or convexity, in order to exploit their structure to develop efficient solution methods. References can be found in Vicente and Calamai (1994); Dempe (2002); Colson et al. (2005, 2007).

We now concentrate on the application of the BP paradigm for price setting problems, which can be seen as sequential games between a company and potential customers. 


\subsection{The pricing problem}

In a pricing problem the leader (first level) sets some taxes or prices for some activities, and the followers (second level) select activities from among taxed and untaxed ones to minimise operating costs. We assume that there are $n_{1}$ taxed and $n_{2}$ untaxed activities. By setting $T \in \mathbb{R}^{n_{1}}$ as the tax vector, $x \in \mathbb{R}^{n_{1}}$ and $y \in \mathbb{R}^{n_{2}}$ as the vectors associated with taxed and untaxed activities respectively, $f$ and $g$ as the objective functions of the leader and the follower respectively, and $\Pi \subset \mathbb{R}^{n_{1}+n_{2}}$ as the feasible solution set, the pricing problem can be formulated as:

$$
\begin{array}{ll}
\max _{T} & f(T, x, y), \\
\text { s.t. } & (x, y) \in \arg \min _{x, y} g(T, x, y), \\
& (x, y) \in \Pi .
\end{array}
$$

This pricing problem arises in several applications. Transportation planning is a typical domain in which examples of such hierarchical structures appear: the first level corresponds to the network operator seeking to improve the performance of the network, and the second level corresponds to network users making their travel choices. This framework fits many applications, such as for example the toll optimisation of highways (this has been carried out for France and Spain), of truck toll systems (in Germany), the pricing of express mail delivery, passenger transportation systems (train, airlines), and various other pricing schemes (hotel rooms, car rental, travel and tourism packages and telecommunications packages). Migdalas (1995) provides a review of bilevel programming problems that arise in transportation, for instance network design, signal setting and origin/destination matrix adjustment problems. These problems usually contain the so-called network equilibrium problem as the second level. Later, an overview of Stackelberg pricing problems applied to networks was carried out by Van Hoesel (2008).

Toll optimisation schemes for highways using bilevel programs have been studied by Labbé et al. (1998), Dewez et al. (2008) and Heilporn et al. (2010b, 2011), with a leader owning the highway and setting tolls on his/her arcs, and the followers travelling either on the highway or on national routes. We discuss this problem in more depth in Sections 1.4 and 1.5 .

A typical problem in revenue management is the product pricing problem, in which a company wants to determine optimal prices for a set of products to maximise the total revenue, given that customers choose maximising their utility (for instance the difference between the benefit they perceive by having the product and the cost of buying it). References on this problem can be found for instance in Shioda et al. (2011). Heilporn et al. (2010a) show that a parallel between this problem and the highway tolling problem described above can be established. More details on this relationship are presented in Section 1.6 
Applications of pricing models to telecommunication networks can be found in Bouhtou et al. (2007b a). In the first paper, the authors approach the pricing problem of a telecommunications operator owning part of a network in which clients want to route their flows at minimum cost, whereas in the second paper they consider a restriction of it, considering that each client utilises at most one of the operator's arcs (as if the operator would own bridges over a river and wanted to optimally price them). They prove that this restricted problem is $\mathcal{A P} \mathcal{X}$-hard. They also propose some preprocessing methodologies to reduce the size of the network on which the model is solved. We discuss these reduction methods in Sections 1.4.6 and 1.4.7.

Brotcorne et al. (2000) consider a bilevel model for the freight tariff-setting problem, where the follower is a shipping company willing to send a prescribed quantity of goods from origin nodes to customers at minimum cost, and the leader is a carrier seeking to maximise its revenue by setting optimal tariffs on the subset of arcs it controls. They assume that the leader is not a dominant player of the market, implying that the total demand is not influenced by the leader's prices. Competitors (i.e. other carriers) do not react in the short term to the leader's prices. Another domain in which this assumption holds is the trucking industry. For given freight rates set by the leader, the shipper's distribution problem is a transshipment problem, whose solution is an assignment of flow on some subtree of the graph. The authors first show how the bilevel model can be reformulated as a single level bilinear program. They then propose metaheuristics that explicitly take into account the structure of the network. In particular, they describe four primal-dual heuristics. The efficiency of these algorithms is calculated by comparing their solutions to optimal ones obtained from a mixed integer reformulation of the model, using a commercial solver. Extensions of these heuristic algorithms have been proposed by Brotcorne et al. (2001). They present and test an algorithmic scheme that can solve toll-setting problems of significant sizes to near optimality, within reasonable computing times.

Brotcorne et al. (2008) consider the problem of pricing in a network together with the design issue: this situation is realistic where the network design can be changed in the short term together with the pricing, for example, in telecommunications networks. In this case, the leader is a telecommunications operator, which wants to simultaneously determine the connections to be opened and the tariff to be applied on them. The followers are users sending flows along cheapest paths joining their respective origins and destinations. Brotcorne et al. (2008) propose a bilevel formulation for this problem and discuss its properties. In particular, they show how the capacity constraints (ensuring that positive flows are routed only on open links, and that they respect capacities) present at the lower level can be moved to the upper level without affecting the optimal solution. From an economic point of view, this behaviour can be interpreted as follows: capacity constraints imposed on the users can be enforced through a suitable and finite tariff schedule, and this can be achieved without affecting the leader's revenue. The authors therefore use this property to develop an efficient solution algorithm, as the 
lower-level problem reduces to a set of independent shortest path problems. Finally they provide some numerical results on both randomly generated and real data.

Toll setting policies are considered by Marcotte et al. (2009) in order to regulate the risk derived by hazardous materials transportation. Often a network design approach is used in the literature to tackle this problem, where there is an authority (e.g. the government), which can forbid the use of some arcs of the network to transport certain types of hazardous materials. Carriers then choose the routes in the resulting network. The objective of both the authority and carriers is to minimise a weighted sum of the risk caused by the hazardous materials transportation and the transportation cost, with different weights. Simpler version may be constructed considering a zero weight for the transportation cost in the authority objective function and/or for the risk factor in the carriers objective function. Bilevel formulations for this hazmat transport network design problem are proposed for instance by Kara and Verter (2004) and Amaldi et al. (2011). Marcotte et al. (2009) propose a new approach for this problem, using tolls to channel the shipments of hazardous materials transportation. In this setting the authority does not design the network forbidding the use of certain arcs, but imposes tolls on them and therefore affects the transportation cost and the route choices. The objective functions of both the authority and carriers remain the same, in a bilevel framework. Marcotte et al. (2009) show that the toll problem is not equivalent to the design problem, unless there is only one shipment to be done. They also point out that toll setting policies give more flexibility compared to network design ones, as they permit to differentiate between carriers. Different formulations for both toll setting and network design versions of the problem are presented, together with efficient solution methods. Computational experiments are carried out on a data set from Western Ontario, Canada.

In Castelli et al. (2013) a bilevel pricing model has been proposed to determine en route charges in the context of Air Traffic Management (ATM). En route charges are tolls applied by national ATM agencies to all flights passing over European countries, as specified by European Commission regulations. The problem is described as bilevel involving one national agency imposing charges on its airspace and seeking to gain the maximum possible revenue. Flights travel between a predetermined pair of airports and choose the cheapest route. In the European airspace, flights are not entirely free to choose their route, as they must pass through a certain number of specific points (latitude, longitude and altitude) prescribed by the national ATM agencies. In practice, a given flight between a pair of airports can typically choose between six to eight different flight paths. These paths may go through different countries, and may involve different distances within each country. The en route charges are calculated proportionally to the distance flown in each country and to the type of aircraft. Each European national ATM agency chooses one toll unit value to charge all air traffic travelling through its airspace. Castelli et al. (2013) propose a sequential solution procedure and test it on some real air traffic data. They show that a national ATM agency could realistically use this approach 
to determine the best en route charge to apply in its airspace. This approach could be extended to other cases of proportional tolls (for instance a per kilometre toll on a road network). We study it into details in Chapter 2.

Cardinal et al. (2011) consider a pricing problem where the follower is looking for the minimum spanning tree on a graph, and the leader owns a subset of arcs and prices them, maximising his/her revenue. This framework applies in telecommunications problems for example, where a company owns and sells several point-to-point connections between locations, and a customer wants to buy a network connecting his/her locations in the form of a spanning tree. The market is composed of the company and its competitors, which own the other connections. Cardinal et al. (2011) prove that this problem is $\mathcal{A P} \mathcal{X}$-hard even if there are only two different cost values on the arcs owned by the competitors. They give an approximation algorithm, provide an integer linear formulation of the problem and study the relation between them.

Finally, this pricing paradigm appears to be an adequate framework for the Principal/Agent Problem (see Van Ackere, 1993). In this problem, widely studied in economics, there is a principal who wants to delegate some task to an agent against reward. The principal optimises his/her utility (e.g. by minimising the reward offered to the agent) whilst ensuring that the agent accepts the task and performs it in a satisfactory way. In the classical example of agency theory, the principal is interpreted as being the owner of a company and the agent its manager, who should be motivated to act in the interest of the principal even when the principal cannot observe the action. In the basic model, the outcome of the task performed by the agent depends on two elements: the level of effort exerted by the agent, which is a disutility for the agent and cannot be observed by the principal, and a random factor, which the agent learns after selecting his/her level of effort and which the principal does not observe. If the agent refuses the task, he/she obtains a certain utility level, which must be smaller than the expected utility level in case of acceptance. For more details on how these elements can be computed and on variations of this basic scheme, we refer the interested reader to Van Ackere (1993). She considers the model with different risk scenarios and different levels of knowledge for the players involved, and also describes some applications of this model in accounting (for budgetary control systems and variable cost allocation), in industrial organisation (for capital structure, disciplining of the product, labour and capital markets and the role of supervision), in marketing (for the relationship between a sales manager and a salesman) and in finance (for the relationship between the shareholders and the manager of a company). Van Ackere (1993) also presents some management science problems in which this framework arises, such as centralised versus decentralised production planning, scheduling of rare resources and selection of batch size. Aside from all the details inherent to each individual real situation to be considered, the Principal/Agent problem could be modelled as a BP pricing problem, where the principal is the leader who wants to determine the minimum reward to offer to one or more agents (followers), such that tasks are guaranteed to be carried out with good 
results.

\subsubsection{The linear pricing problem}

We now focus on pricing problems in which the objective functions are bilinear: both levels objective functions are linear once the other level choices are fixed. All constraints are linear. The second level objective function has coefficient vectors $c_{1} \in \mathbb{R}^{n_{1}}$ and $c_{2} \in \mathbb{R}^{n_{2}}$, which represent the fixed costs of taxed and untaxed activities respectively. Matrices $A_{1} \in \mathbb{R}^{m \times n_{1}}$ and $A_{2} \in \mathbb{R}^{m \times n_{2}}$, and vector $b \in \mathbb{R}^{m}$, represent the coefficients of the $m$ constraints of the follower's feasible solution set. The (bi)linear pricing problem can be modelled as follows:

$$
\begin{array}{rl}
\max _{T} & T x, \\
\text { s.t. }(x, y) \in \arg \min _{x, y} & \left(c_{1}+T\right) x+c_{2} y, \\
\text { s.t. } & A_{1} x+A_{2} y=b, \\
& x, y \geq 0 .
\end{array}
$$

To guarantee the existence of a bounded solution, we assume that the follower's feasible solution set $\Pi_{1}=\left\{(x, y): A_{1} x+A_{2} y=b, x, y \geq 0\right\}$ is non-empty and bounded, and that the follower set of feasible solutions using only untaxed activities $\Pi_{2}=\left\{y: A_{2} y=b, y \geq 0\right\}$ is non-empty. In fact, if $\Pi_{1}$ is non-empty and bounded, the second level problem will always have a finite solution. The non-emptiness of $\Pi_{2}$ guarantees the existence of a tax-free solution for the follower, which is necessary to prevent the leader from imposing an infinite tax on his/her activities, leading to an infinite revenue.

To illustrate the concepts introduced so far, we consider the particular case where the second level has only two decision variables, meaning that the followers can choose between a taxed activity and a free one, and the leader has one tax value $T$ to determine. The formulation is the same as described above, with decision variables $T, x, y \in \mathbb{R}$, parameters $c_{1}, c_{2} \in \mathbb{R}$ and vectors $A_{1}, A_{2}, b \in \mathbb{R}^{m}$. In such a case a graphical representation of the problem can be provided (see Figure 1.1) and the optimal solution can be found using a relatively straightforward procedure.

In Figure 1.1(a) the second level objective function is represented, with the set of feasible solutions $\Pi$. Each vertex of $\Pi$ represents a potential optimal solution for the follower. From linear programming theory, one can easily conclude that a vertex of $\Pi$ is optimal if the opposite of the objective function coefficient vector $\left(-\left(c_{1}+T\right),-c_{2}\right)$ belongs to the cone generated by the coefficient vectors of the active constraints at that vertex. This allows one to determine, for each vertex, the values of $T$ for which it is optimal. For instance, vertex $\left(x^{0}, y^{0}\right)$ is optimal for $T \in\left[0, T_{0}\right],\left(x^{1}, y^{1}\right)$ is optimal for $T \in\left[T_{0}, T_{1}\right]$, and so on. The first level objective function $T x$ is depicted in terms of $T$ in Figure 1.1(b), One can observe that this function is discontinuous and piecewise linear 
with slopes $x^{0}, x^{1}$, etc. The optimal solution in this simple example is given by $T_{1}$ and $\left(x^{1}, y^{1}\right)$. For further details on this case, we refer the interested reader to Labbe et al. (1998).

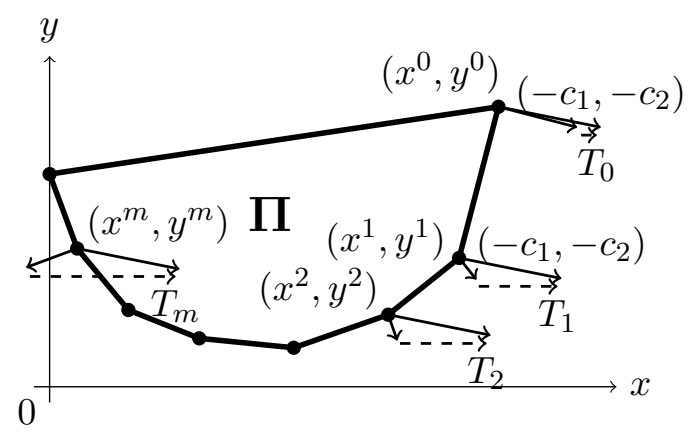

(a) Second level - Feasible solutions

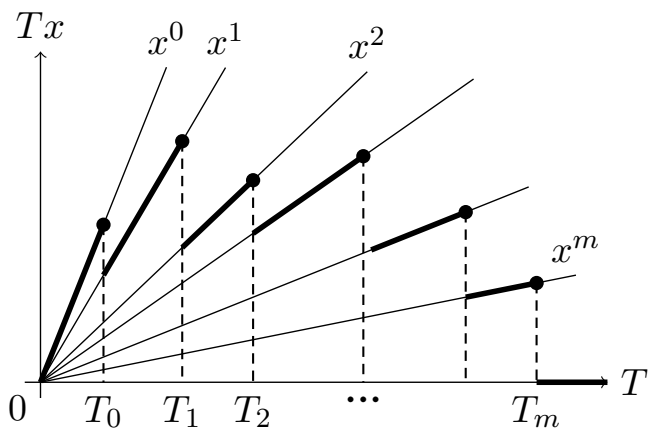

(b) First level - Objective function (in bold)

Figure 1.1: Graphical example of the objective functions of the (bi)linear pricing problem in a two-dimensional case (Labbé et al., 1998)

\subsection{The Network Pricing Problem}

The Network Pricing Problem (NPP) is a pricing problem on a network, with an authority which owns a subset of arcs and imposes tolls on them, and users who travel on the network. The authority is the leader who wants to maximise his/her revenue, and network users are the followers who want to minimise their costs, and so will always travel on their minimum cost path.

The transportation network is defined as a set of nodes linked by a set of (directed) arcs. We define $\mathcal{N}$ as the set of nodes $i, \mathcal{A}_{1}$ as the set of toll arcs (i.e. arcs owned by the leader on which he/she can impose tolls) and $\mathcal{A}_{2}$ as the set of other or toll free arcs. We denote as $a$ the generic arc. The set $\mathcal{K}$ represents the commodities $k$, which are groups of network users travelling from an origin to a destination. We assume that for each commodity there exists a toll free path between its origin and destination, i.e. a path which does not pass through any of the arcs owned by the authority, as otherwise the authority could impose an infinite toll on his/her arcs to obtain infinite revenues.

As an example, we consider the network depicted in Figure 1.2 in which a single commodity wants to travel from A to E. The authority owns arcs B-C and D-E (dashed arcs), and fixed costs on arcs are reported on the graph. The toll free path is A-C-E with cost 22 , so this is an upper bound for the total travel cost the commodity is willing to pay. A corresponding lower bound is given by the cost of the shortest path on the network if the authority imposes zero tolls. This path is A-B-C-D-E with a cost of 6 . Hence, an upper bound for the authority's revenue can be calculated as the cost of the shortest toll free path minus the cost of the shortest path if all tolls are set to zero (in this example: $22-6=16$ ). The toll free path cost can be computed as the cost of the 
shortest path on the network in which all tolls are set to infinity. By denoting by $\gamma^{k}(\mathbf{T})$ the cost of the shortest path for commodity $k$ and toll vector $\mathbf{T}$, this upper bound on the authority's revenue can be written as $U B^{k}=\gamma^{k}(\infty)-\gamma^{k}(0)$. This bound is not always reached, as can been seen in this example: the toll on arc B-C must be at most 5 , and on arc D-E at most 10 . So the authority's revenue will never exceed 15 . In fact, these values provide an optimal solution.

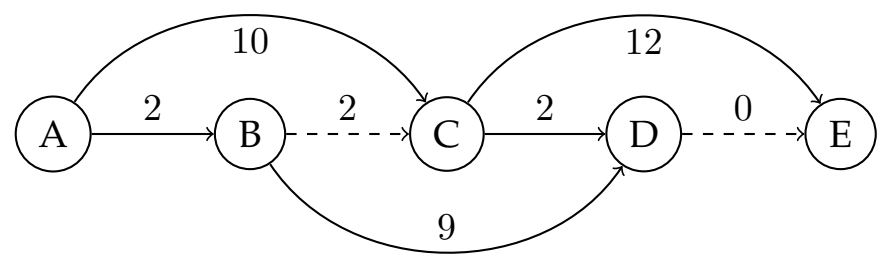

Figure 1.2: Example of a NPP (from Dewez, 2004)

If tolls are allowed to be negative, the model can deal with subsidies. Even if this situation is not intuitive, the following example shows that an optimal solution may require negative tolls on certain arcs. In Figure 1.3 we report a network in which a single user wants to travel from A to D. The leader owns arcs A-B, B-C and C-D which have zero fixed cost, and then there are toll free $\operatorname{arcs} A-C, A-D$ and B-D. As previously described an upper bound on leader's revenue is 6 . With some simple calculations, an optimal solution is $T_{A-B}=T_{C-D}=4$ and $T_{B-C}=-2$, for a total revenue of 6 (as it is equal to the upper bound, the optimality is guaranteed).

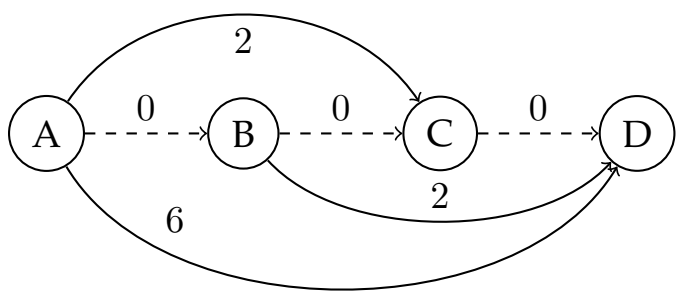

Figure 1.3: Example of a NPP with negative tolls (from Labbé et al. 1998)

In this thesis we suppose tolls to be always non-negative, but most of the results can be generalised to arbitrary tolls.

There are also some cases where the follower has multiple optimal solutions for a given toll vector. Here we consider that in such cases the commodities take the choice which is most profitable for the leader. This assumption is not restrictive, because the leader could modify some tolls of his/her most profitable solution by a small value, making that solution the only optimal one for the follower. 


\subsubsection{Arc pricing}

In this section, we introduce the definition of "arc pricing" in contrast to the "path pricing" that will be introduced in Section 1.5. meaning that the leader imposes a toll on each of his/her arcs, and these values can be different.

We first introduce some notation. For each commodity $k \in \mathcal{K}$, let $\eta^{k}$ be its demand and $o^{k}$ and $d^{k}$ be its origin and destination respectively. Moreover, we define $c_{a}$ as the travel cost on arc $a \in \mathcal{A}_{1} \cup \mathcal{A}_{2}$. The leader wants to set a toll $T_{a}$ on each toll arc $a \in \mathcal{A}_{1}$, such that his/her total revenue is maximum, and followers will seek their minimum cost path on the network, fixing flow variables $x_{a}^{k}$ on toll arcs, and $y_{a}^{k}$ on toll free arcs (these variables are equal to 1 if commodity $k$ uses arc $a, 0$ otherwise). Later in the description, when needed for the sake of clarity, we will also use the notation $(i, j)$ to indicate an arc $a$ of the network, where $i$ is the tail node and $j$ the head node of the arc. The NPP for the arc pricing can therefore be modelled as follows:

$$
\begin{aligned}
\max _{T \geq 0} & \sum_{a \in \mathcal{A}_{1}} T_{a} \sum_{k \in \mathcal{K}} \eta^{k} x_{a}^{k}, \\
\text { s.t. }(x, y) \in \arg \min _{x, y} & \sum_{k \in \mathcal{K}}\left(\sum_{a \in \mathcal{A}_{1}}\left(c_{a}+T_{a}\right) x_{a}^{k}+\sum_{a \in \mathcal{A}_{2}} c_{a} y_{a}^{k}\right), \\
\text { s.t. } & \sum_{a \in i^{+}}\left(x_{a}^{k}+y_{a}^{k}\right)-\sum_{a \in i^{-}}\left(x_{a}^{k}+y_{a}^{k}\right)=b_{i}^{k} \\
& x_{a}^{k}, y_{a}^{k} \geq 0
\end{aligned} \quad \forall k \in \mathcal{K}, \forall i \in \mathcal{N} \text {, }
$$

where $i^{-}$and $i^{+}$denote the sets of arcs with $i$ as head or tail respectively, and $b_{i}^{k}$ is equal to -1 if $i$ is the origin node of commodity $k, 1$ if it is the destination node, and 0 otherwise. As the second level is a shortest path problem (Equations $1.4 \mathrm{~b}, 1.4 \mathrm{c}$ ) and $(1.4 \mathrm{~d})$ ), whose linear programming formulation has the total unimodularity property, there is no need for integrality constraints on the decision variables. This bilevel NPP for a multicommodity network was first introduced by Labbé et al. (1998).

\subsubsection{The case of a single toll arc}

The case of a NPP where the authority owns only one arc $a$ is relatively straightforward, and can be solved in polynomial time (Labbé et al., 1998). We define $T$ as the toll value the leader can impose on the single toll arc, and $\gamma^{k}(T)$ as the cost of the shortest path for the commodity $k$ for a given value of $T$. We set the upper bound of the toll that can be imposed by the leader for commodity $k$ as $M^{k}=\gamma^{k}(\infty)-\gamma^{k}(0)$. We then sort $M^{k}$ quantities for all commodities in decreasing order. We assume that the order is 
$M^{1} \geq M^{2} \geq \ldots \geq M^{|\mathcal{K}|}$, where $|\mathcal{K}|$ is the number of commodities. For any toll value $T$ which is not equal to one of the values in this $M^{k}$ sequence, we can increase the toll by some $\epsilon>0$ and achieve a higher revenue. Thus, the optimal value of $T$ is equal to one of the $M^{k}$ values. Moreover, for a toll value $M^{i}(i \in\{1 \ldots|\mathcal{K}|\})$ only commodities $k \leq i$ (for which $M^{k} \geq M^{i}$ ) will choose the toll arc. The leader revenue function is:

$$
\mathcal{R}\left(M^{i}\right)=M^{i} \sum_{k \leq i} \eta^{k}
$$

The leader will choose the toll value that maximises his/her revenue, so the optimal solution will be:

$$
T^{\star}=M^{i^{\star}}, \text { such as } i^{\star}=\arg \max _{i \in\{1 \ldots|\mathcal{K}|\}} \mathcal{R}\left(M^{i}\right) .
$$

The leader revenue function is shown in Figure 1.4. It is a piecewise linear function, with discontinuities at $M^{i}$ values. In each interval the function is described by a straight line whose slope is given by the cumulative demand of commodities, which will choose the toll arc for that $M^{i}$ value, equal to $\sum_{k \leq i} \eta^{k}$.

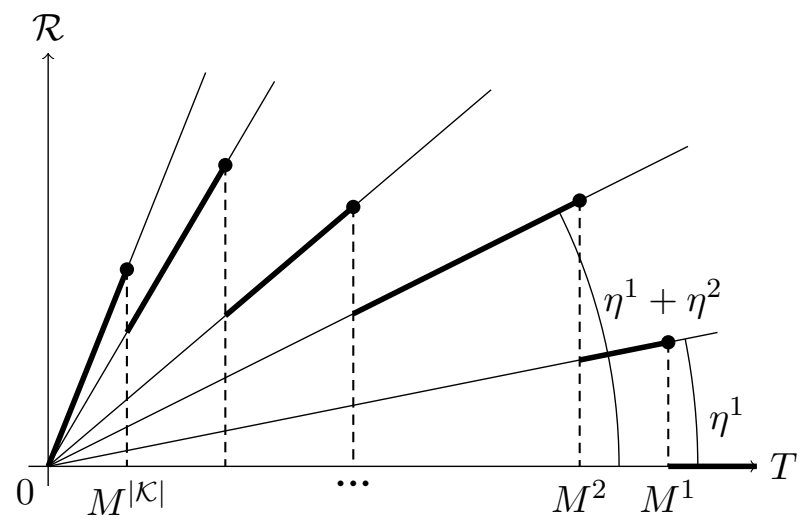

Figure 1.4: Leader revenue (in bold) in the case of a single toll arc pricing problem

We therefore know that for a fixed $T \in \mathbb{R}$, commodity $k$ will use the toll arc (i.e. $\left.x^{k}=1\right)$ only if $M^{k} \geq T$ and that the optimal toll value lies in $\left\{M^{k}: k \in \mathcal{K}\right\} \cup\{0\}$. We are easily able to calculate the leader's revenue for these toll values and take the one maximising it.

In Dewez (2004), a simple formulation was proposed for this single toll arc case. After ordering commodities according to decreasing $M^{k}$ values, define a cumulative demand as $S^{k}=\sum_{i=1}^{k} \eta^{i}$, for all $k \in \mathcal{K}$. We define a binary variable $\omega^{k}$ for each $k \in \mathcal{K}$, which is equal to 1 if the chosen toll value is $M^{k}, 0$ otherwise. The single toll arc problem can be formulated as follows: 


$$
\begin{array}{ll}
\max _{\omega} & \sum_{k \in \mathcal{K}} S^{k} M^{k} \omega^{k}, \\
\text { s.t. } & \sum_{k \in \mathcal{K}} \omega^{k}=1, \\
& \omega^{k} \in\{0,1\} \quad \forall k \in \mathcal{K} .
\end{array}
$$

It is straightforward to see that binary conditions on variables (constraints $(1.7 \mathrm{c})$ ) can be relaxed, leading to a polynomial formulation.

\subsubsection{Complexity}

In Labbé et al. (1998), the authors prove that the general problem is $\mathcal{N} \mathcal{P}$-hard, while some particular instances are polynomially solvable, e.g. the single toll arc case described above. Roch et al. (2005) strengthen this result, proving the following proposition.

Proposition 1.4.1 (NPP is strongly $\mathcal{N} \mathcal{P}$-hard). The NPP is strongly $\mathcal{N} \mathcal{P}$-hard, even for one single commodity and/or when negative tolls are allowed (Roch et al., 2005).

Proof. First of all let us define the decision problem: given an instance of the NPP and a constant $\mathcal{R}$, does a toll vector $T$ exist such that $T x \geq \mathcal{R}$, and such that $(x, y)$ is an optimal flow for the second level in reaction to $T$ ? Given a fixed toll vector $T$, optimal flow variables can easily be determined with a shortest path algorithm. We can check in polynomial time if the toll vector satisfies the above condition. The NPP problem is therefore in $\mathcal{N} \mathcal{P}$.

Roch et al. (2005) use a reduction from 3-SAT (see for example Garey and Johnson, 1979) to the NPP. Consider a 3-SAT formula $F=\bigwedge_{i=1}^{m}\left(l_{i, 1} \vee l_{i, 2} \vee l_{i, 3}\right)$, with $m$ clauses and three literals per clause, $l_{i, j}$ for $j=1,2,3$. Each literal corresponds to a variable $x_{1}, \ldots, x_{n}$ or to its negation. Each clause of a 3-SAT formula is represented as a subnetwork, where there are three toll arcs representing the three literals of the clause and one toll free arc of cost 1 (see Figure 1.5). Subnetworks are then connected by two arcs, one toll arc and one toll free arc of cost 2 (see the example of Figure 1.6). All toll arcs have zero fixed cost. There is one single user who wants to travel from node $s$ to node $t$.

The idea is that if the optimal path goes through a toll arc, then the corresponding literal is true. If $F$ is satisfiable, the optimal path has to go through exactly one toll arc per subnetwork, meaning that one literal per clause is true. Moreover the assignment of variables has to be consistent, such that the optimal path does not include a variable and its negation. To prevent this from occurring, Roch et al. (2005) add an interclause toll free arc with fixed cost of 1 between the toll arcs of each pair of literals corresponding to a variable and its negation. These arcs guarantee that inconsistent paths are suboptimal. In Figure 1.6 we show the network constructed with these rules for the formula $F=$ $\left(x_{1} \vee x_{2} \vee \overline{x_{3}}\right) \wedge\left(\overline{x_{2}} \vee x_{3} \vee \overline{x_{4}}\right) \wedge\left(\overline{x_{1}} \vee x_{3} \vee x_{4}\right)$. 


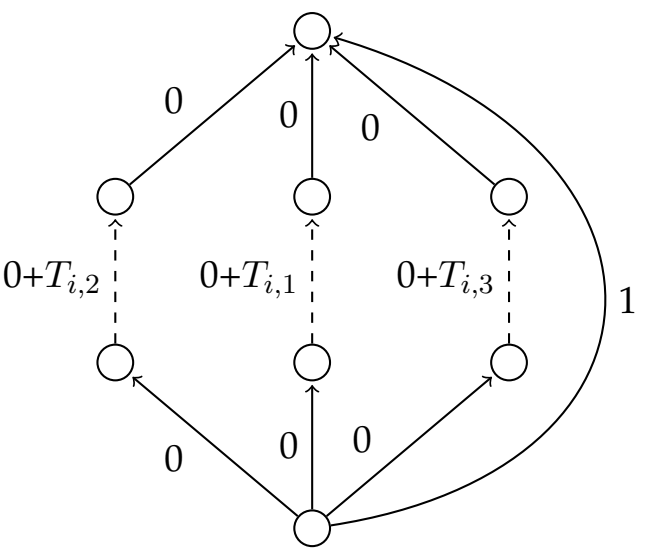

Figure 1.5: Strong $\mathcal{N} \mathcal{P}$-hard proof for the NPP: subnetwork for one clause $\left(l_{i, 1} \vee l_{i, 2} \vee l_{i, 3}\right)$

The length of the shortest toll free path in such a network is equal to $m+2(m-1)=$ $3 m-2$, and the length of the shortest path with zero tolls is equal to 0 . The leader's revenue is therefore bounded from above by $3 m-2$. Roch et al. (2005) prove that $F$ is satisfiable if and only if the optimal solution value of the NPP is equal to this bound.

Let us initially assume that the NPP has an optimal solution value of $3 m-2$. To reach this bound, the optimal path has to go through one toll arc per subnetwork, and tolls have to be set to 1 on the corresponding literals, to $C+1$ on other literals (where $C$ is the sum of all fixed arc costs of the network, large enough that the commodity does not take these arcs) and to 2 on toll arcs linking subnetworks. The optimal path cannot include a variable and its negation because, if this were the case, the interclause toll free arc would impose an upper bound of 1 on tolls of arcs linking subnetworks, which is in contradiction with the optimal value of 2 for tolls on these arcs. Therefore, the optimal path corresponds to a consistent assignment and $F$ is satisfiable.

Consider now that $F$ is satisfiable. This means that at least one literal per clause is true. It is then possible to take into account the path going through these literals. Moreover, as the assignment is consistent, there are no interclause toll free arcs limiting the revenue. Thus, the upper bound of $3 m-2$ is reached on this path.

Finally, there are at most $10 m+2(m-1)+(3 m)^{2}$ arcs in the network constructed with these rules, such that every 3-SAT instance is reducible to an NPP instance in polynomial time.

For the case of a single commodity, Roch et al. (2005) also provide a polynomial approximation algorithm, with an approximation factor of $\alpha=\frac{1}{2} \log _{2}\left|\mathcal{A}_{1}\right|+1$, where $\left|\mathcal{A}_{1}\right|$ denotes the number of toll arcs in the network. This means that such an algorithm is guaranteed to compute a feasible solution with an objective value of at least $O P T / \alpha$, where $O P T$ is the optimal leader's revenue. 


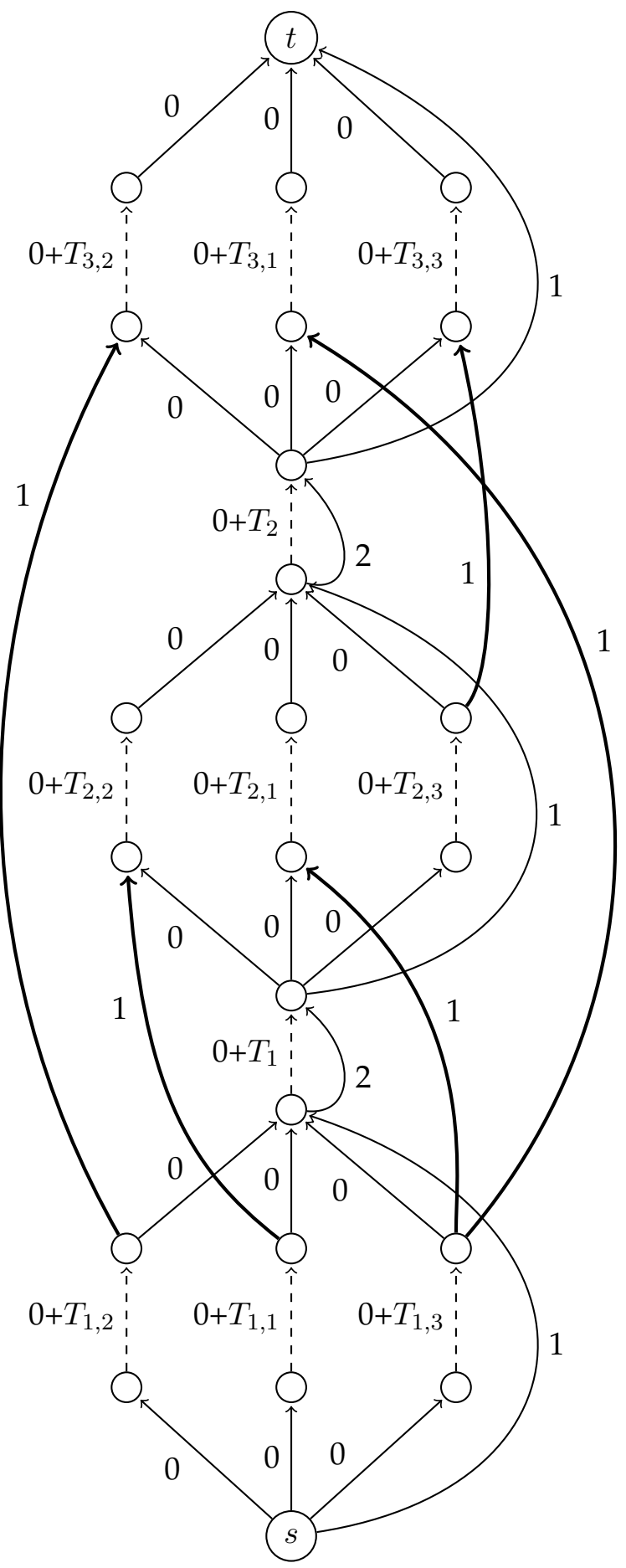

Figure 1.6: Strong $\mathcal{N} \mathcal{P}$-hard proof for the NPP: network for the formula $\left(x_{1} \vee x_{2} \vee \overline{x_{3}}\right) \wedge\left(\overline{x_{2}} \vee x_{3} \vee \overline{x_{4}}\right) \wedge\left(\overline{x_{1}} \vee x_{3} \vee x_{4}\right)$ (from Roch et al., 2005) 
Recently, Joret (2011) shows that the single commodity NPP is $\mathcal{A P} \mathcal{X}$-hard, with a reduction from 3-SAT-5 (i.e. 3-SAT where each variable appears in exactly 5 clauses) and a network structure similar to the one developed by Roch et al. (2005) for the $\mathcal{N} \mathcal{P}$ hardness proof.

\subsubsection{One level MIP formulation}

Labbé et al. (1998) show how the lower level optimisation problem (Equations $1.4 \mathrm{~b}$ to $(1.4 \mathrm{~d})$ ) can be replaced by its primal and dual constraints (Equations $1.8 \mathrm{~b}),(1.8 \mathrm{c}$ ) and $(1.8 \mathrm{~d})$ ), and its optimality conditions (Equation (1.8e)), stating that the primal and dual objective functions of each commodity must be equal. This yields the following singlelevel optimisation problem $\left(\lambda_{i}, \forall i \in \mathcal{N}\right.$, are the dual variables associated to constraints $(1.4 \mathrm{c})$ :

$$
\begin{array}{rlr}
\max _{T, x, y, \lambda} & \sum_{a \in \mathcal{A}_{1}} T_{a} \sum_{k \in \mathcal{K}} \eta^{k} x_{a}^{k}, \\
\text { s.t. } & \sum_{a \in i^{+}}\left(x_{a}^{k}+y_{a}^{k}\right)-\sum_{a \in i^{-}}\left(x_{a}^{k}+y_{a}^{k}\right)=b_{i}^{k} & \forall k \in \mathcal{K}, \forall i \in \mathcal{N}, \\
& \lambda_{i}^{k}-\lambda_{j}^{k} \leq c_{a}+T_{a} & \forall k \in \mathcal{K}, \forall a \in \mathcal{A}_{1}, \forall i, j \in \mathcal{N}, \\
& \lambda_{i}^{k}-\lambda_{j}^{k} \leq c_{a} & \forall k \in \mathcal{K}, \forall a \in \mathcal{A}_{2}, \forall i, j \in \mathcal{N}, \\
& \sum_{a \in \mathcal{A}_{1}}\left(c_{a}+T_{a}\right) x_{a}^{k}+\sum_{a \in \mathcal{A}_{2}} c_{a} y_{a}^{k}=\lambda_{o_{k}}^{k}-\lambda_{d_{k}}^{k} & \forall k \in \mathcal{K}, \\
& x_{a}^{k}, y_{a}^{k} \geq 0 \\
& T_{a} \geq 0 & \forall k \in \mathcal{K}, \forall a \in \mathcal{A}_{1} \cup \mathcal{A}_{2}, \\
\forall a \in \mathcal{A}_{1} .
\end{array}
$$

This problem is non-linear, as the objective function (1.8a) and constraints (1.8e) contain the bilinear term $T_{a} x_{a}^{k}$. A linearisation can be done by introducing the set of decision variables $T_{a}^{k}=T_{a} x_{a}^{k}$, which represent the effective price paid by commodity $k$ on arc $a$ (equal to $T_{a}$ if the commodity is using the arc, or 0 otherwise). The necessity of linking new variables with the old ones induces the addition of the new constraints $(1.9 \mathrm{f}),(1.9 \mathrm{~g})$ and $1.9 \mathrm{~h}$, where $M_{a}^{k}$ and $N_{a}$ are so-called "big M" constants. In particular, constraints (1.9f) impose that $T_{a}^{k}=0$ if the arc $a$ is not used by commodity $k$, and constraints $1.9 \mathrm{~g}$, and $1.9 \mathrm{~h}$ set $T_{a}^{k}=T_{a}$ if the arc is used, $\forall k \in \mathcal{K}, \forall a \in \mathcal{A}_{1}$. The linearised model is a Mixed Integer Problem (MIP), whose formulation is as follows: 


$$
\begin{aligned}
\max _{T, x, y, \lambda} & \sum_{a \in \mathcal{A}_{1}} \sum_{k \in \mathcal{K}} \eta^{k} T_{a}^{k}, \\
\text { s.t. } & \sum_{a \in i^{+}}\left(x_{a}^{k}+y_{a}^{k}\right)-\sum_{a \in i^{-}}\left(x_{a}^{k}+y_{a}^{k}\right)=b_{i}^{k} \\
& \lambda_{i}^{k}-\lambda_{j}^{k} \leq c_{a}+T_{a} \\
& \lambda_{i}^{k}-\lambda_{j}^{k} \leq c_{a} \\
& \sum_{a \in \mathcal{A}_{1}}\left(c_{a} x_{a}^{k}+T_{a}^{k}\right)+\sum_{a \in \mathcal{A}_{2}} c_{a} y_{a}^{k}=\lambda_{o_{k}}^{k}-\lambda_{d_{k}}^{k} \\
& T_{a}^{k} \leq M_{a}^{k} x_{a}^{k} \\
& T_{a}-T_{a}^{k} \leq N_{a}\left(1-x_{a}^{k}\right) \\
& T_{a}^{k} \leq T_{a} \\
& x_{a}^{k} \in\{0,1\} \\
& y_{a}^{k} \geq 0 \\
& T_{a}^{k} \geq 0 \\
& T_{a} \geq 0
\end{aligned}
$$$$
\forall k \in \mathcal{K}, \forall i \in \mathcal{N}
$$$$
\forall k \in \mathcal{K}, \forall a \in \mathcal{A}_{1}, \forall i, j \in \mathcal{N},
$$$$
\forall k \in \mathcal{K}, \forall a \in \mathcal{A}_{2}, \forall i, j \in \mathcal{N} \text {, }
$$

$\forall k \in \mathcal{K}$

$\forall k \in \mathcal{K}, \forall a \in \mathcal{A}_{1}$,

$\forall k \in \mathcal{K}, \forall a \in \mathcal{A}_{1}$,

$\forall k \in \mathcal{K}, \forall a \in \mathcal{A}_{1}$,

$\forall k \in \mathcal{K}, \forall a \in \mathcal{A}_{1}$,

$\forall k \in \mathcal{K}, \forall a \in \mathcal{A}_{2}$,

$\forall k \in \mathcal{K}, \forall a \in \mathcal{A}_{1}$,

$$
\forall a \in \mathcal{A}_{1} \text {. }
$$

Note that variables $x_{a}^{k}$ must be binary for this reformulation to be valid.

Dewez et al. (2008) present several families of valid inequalities in order to reinforce the linear relaxation.

\subsubsection{Tight values for "big $M$ and $N$ "}

In order to make the linear relaxation of the previous problem as tight as possible, $M_{a}^{k}$ and $N_{a}$ should be set to the smallest values that ensure a valid formulation. As we will see later from the numerical results, a good choice of these constants can significantly improve the performance of solution methods. Dewez et al. (2008) show how to calculate valid values for them. In practice, the constant $M_{a}^{k}$ represents an upper bound on the toll value that commodity $k$ is willing to pay to use arc $a$. The constant $N_{a}$ represents the maximum value of $T_{a}$ that can be imposed on toll arc $a$ so that at least one commodity uses that arc.

Let us extend the meaning of the shortest path cost function by introducing origin and destination indices: $\gamma_{i, j}^{k}(T)$ represents the cost of the shortest path for commodity $k$ from node $i$ to node $j$, with tolls set to $T$. In particular, $\gamma_{i, j}^{k}(\infty)$ represents the cost of the shortest toll free path from $i$ to $j$, and $\gamma_{i, j}^{k}(0)$ the cost of the shortest path if tolls are all set to zero. Consider now one toll arc $a$, with fixed cost $c_{a}$, and let us denote its tail and head nodes by $t(a)$ and $h(a)$ respectively. Recall also that $o^{k}$ and $d^{k}$ are the origin and destination nodes of commodity $k$. One feasible value for $M_{a}^{k}$ can be calculated as the cost of the shortest toll free path from $t(a)$ to $h(a)$ minus the fixed cost $c_{a}$. Other feasible values can be similarly obtained using the paths going from the origin node $o^{k}$ to the 
head node $h(a)$, the paths going from the tail node $t(a)$ to the destination node $d^{k}$ and finally the paths going from the origin node $o^{k}$ to the destination node $d^{k}$. Obviously the best value for $M_{a}^{k}$ will be the smallest one among the four. In Figure 1.7, we show all the paths that occur in the calculation of the four bounds for one toll arc (the dashed arc) and one commodity. Wavy arcs represent shortest paths on the original network.

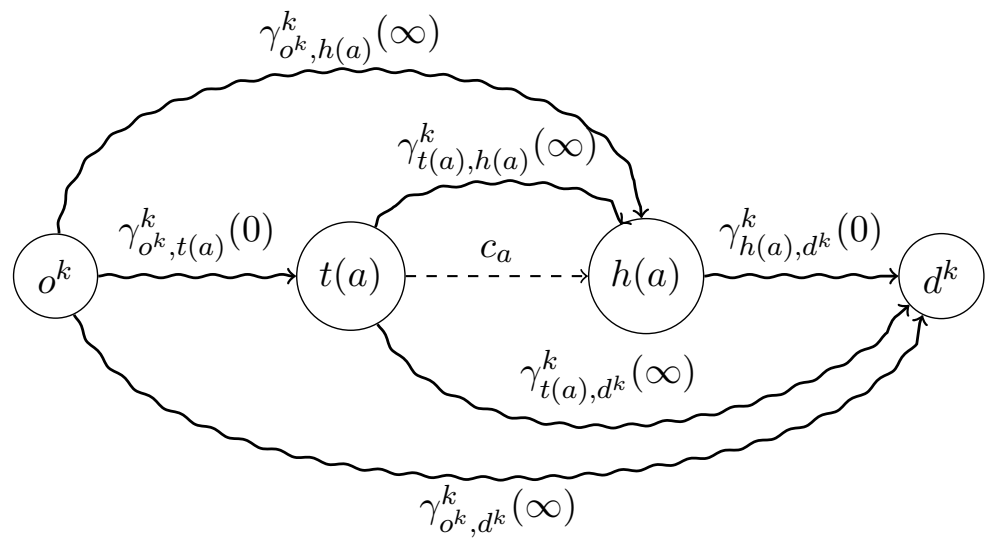

Figure 1.7: Paths that occur in the calculation of valid values for "big M"

The following expression, which translates the reasoning just explained, gives a valid value for $M_{a}^{k}$ :

$$
\begin{aligned}
& \max \left\{0, \min \left\{\gamma_{t(a), h(a)}^{k}(\infty)-c_{a},\right.\right. \\
& \gamma_{o^{k}, h(a)}^{k}(\infty)-\gamma_{o^{k}, t(a)}^{k}(0)-c_{a}, \\
& \gamma_{t(a), d^{k}}^{k}(\infty)-\gamma_{h(a), d^{k}}^{k}(0)-c_{a}, \\
& \left.\left.\gamma_{o^{k}, d^{k}}^{k}(\infty)-\gamma_{o^{k}, t(a)}^{k}(0)-\gamma_{h(a), d^{k}}^{k}(0)-c_{a}\right\}\right\} \text {. }
\end{aligned}
$$

Note that none of the feasible values dominates any other, as shown by the example with the four networks in Figure 1.8. In each network there is one commodity travelling from node 1 to node 5 , toll arcs are dashed and fixed costs are reported in the figure.

Table 1.2 reports all these feasible values of $M_{a}$ for the four networks in Figure 1.8 . The dominant, i.e. smallest, value for each network is represented in bold in the table, and we can see that the dominant bound is different for each of them. Since we assume the existence of a toll free path from the origin to the destination for each commodity, at least the fourth bound $M 4$ is always finite.

Furthermore, for each toll arc $a$, it is easy to see that a valid value for $N_{a}$ is the largest $M_{a}^{k}$ for all commodities, i.e. $N_{a}=\max _{k \in \mathcal{K}} M_{a}^{k}$ (see Dewez et al. 2008).

Computational experiments (see Section 1.4.8) show that using these sharp values for $M_{a}^{k}$ and $N_{a}$ allows us to halve the size of the gap between the values of the optimal integer solution and of the optimal solution of the linear relaxation. Furthermore, some instances were not solvable with arbitrarily large values for $M_{a}^{k}$ and $N_{a}$, whilst solutions were found using the sharp values presented above. 


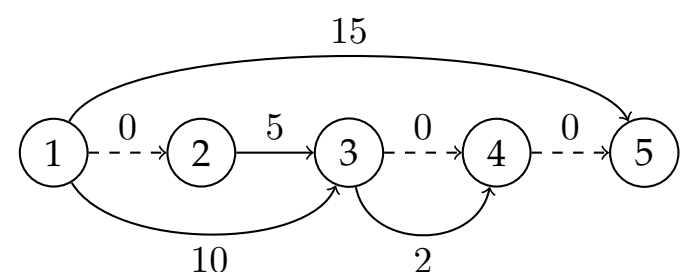

(a) Example of a network in which the first bound is the tightest

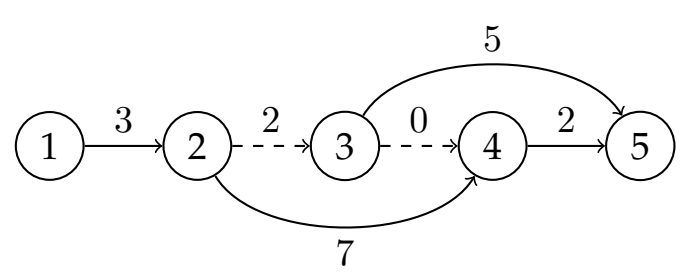

(c) Example of a network in which the third bound is the tightest

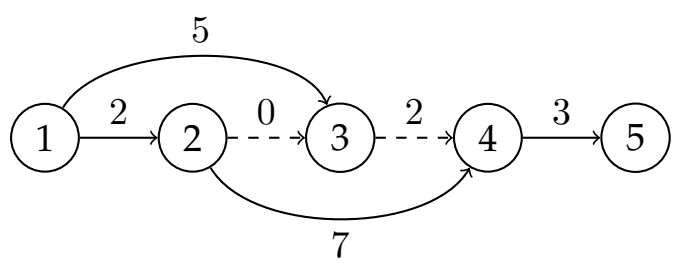

(b) Example of a network in which the second bound is the tightest

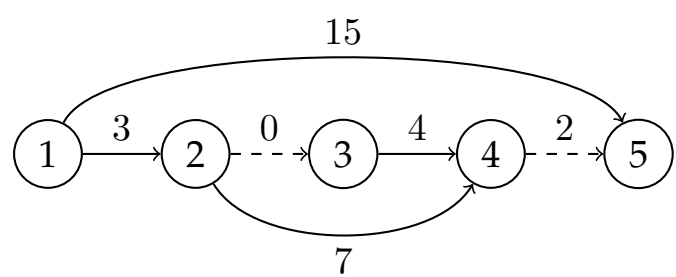

(d) Example of a network in which the fourth bound is the tightest

Figure 1.8: Example of networks for the calculation of valid values on "big M" ((b),(c) and (d) from Dewez, 2004)

\begin{tabular}{l|c|c|c|c|} 
& $\begin{array}{c}\text { Network (a) } \\
\text { toll } \operatorname{arc}(3,4)\end{array}$ & $\begin{array}{c}\text { Network (b) } \\
\text { toll } \operatorname{arc}(2,3)\end{array}$ & $\begin{array}{c}\text { Network (c) } \\
\text { toll arc (3,4) }\end{array}$ & $\begin{array}{c}\text { Network (d) } \\
\text { toll } \operatorname{arc~}(2,3)\end{array}$ \\
\hline$M 1=\gamma_{t(a), h(a)}^{k}(\infty)-c_{a}$ & $\mathbf{2}$ & $\infty$ & $\infty$ & $\infty$ \\
\hline$M 2=\gamma_{o^{k}, h(a)}^{k}(\infty)-\gamma_{o^{k, t(a)}}^{k}(0)-c_{a}$ & 7 & $\mathbf{3}$ & 5 & $\infty$ \\
\hline$M 3=\gamma_{t(a), d^{k}}^{k}(\infty)-\gamma_{h(a), d^{k}}^{k}(0)-c_{a}$ & $\infty$ & 5 & $\mathbf{3}$ & $\infty$ \\
\hline$M 4=\gamma_{o^{k}, d^{k}}^{k}(\infty)-\gamma_{o^{k}, t(a)}^{k}(0)-c_{a}-\gamma_{h_{(a), d^{k}}^{k}(0)}$ & 10 & 5 & 5 & $\mathbf{6}$ \\
\hline
\end{tabular}

Table 1.2: Feasible values for $M_{a}$ for the four networks in Figure 1.8 (dominant values in bold)

\subsubsection{The Shortest Path Graph Model}

The Shortest Path Graph Model (SPGM), proposed by Bouhtou et al. (2007b), is a reformulation of the NPP that reduces the practical size of the original network. In theory, this reformulation does not necessarily yield to a smaller network, but Bouhtou et al. (2007b) conduct numerical experiments showing improvements to the resolution of their models. From a computational time point of view, the SPGM generation can be rather time consuming, especially for large networks, but algorithms can subsequently solve the instances more quickly than would otherwise be the case. Details of numerical experiments for both realistic and random networks are reported in Bouhtou et al. (2007b).

The SPGM exploits the following two observations:

(i) the (shortest) path selected by each commodity constitutes an alternating sequence of toll arcs and subpaths containing only toll free arcs - see Figure 1.9. 
(ii) each subpath linking the head $h\left(a_{i}\right)$ and the tail $t\left(a_{i+1}\right)$ of two consecutive toll $\operatorname{arcs} a_{i}$ and $a_{i+1}$ of a path selected by a commodity $k$ has a minimum toll free cost given by $\gamma_{h\left(a_{i}\right), t\left(a_{i+1}\right)}^{k}(\infty)$.

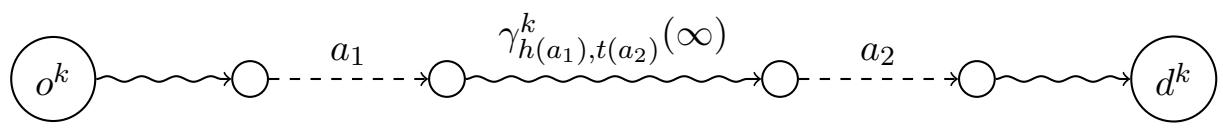

Figure 1.9: Example of the structure of different possible paths in a network with toll arcs

By following this reasoning we can construct a SPGM for each commodity. Let $\mathcal{G}=\left(\mathcal{N}, \mathcal{A}_{1} \cup \mathcal{A}_{2}\right)$ be the original graph, we then define the new SPGM graph as $\mathcal{G}^{k}=$ $\left(\mathcal{N}^{k}, \mathcal{A}_{1}^{k} \cup \mathcal{A}_{2}^{k}\right), \forall k \in \mathcal{K}$. The set of toll arcs $\mathcal{A}_{1}^{k}$ is equal to $\mathcal{A}_{1}$. Then the set of toll free $\operatorname{arcs} \mathcal{A}_{2}^{k}$ is constructed as follows for each commodity $k$. First, $\mathcal{A}_{2}^{k}$ contains a toll free arc between the origin node $o^{k}$ and the destination node $d^{k}$. Then, for two toll arcs $a_{1}=\left(i_{1}, j_{1}\right)$ and $a_{2}=\left(i_{2}, j_{2}\right)$, a toll free arc from $j_{1}$ to $i_{2}$ is added to $\mathcal{A}_{2}^{k}$ if there is a toll free path in $\mathcal{G}$ between them. Finally, a toll free arc from the origin node to the tail of a toll arc is also added to $\mathcal{A}_{2}^{k}$ if there is a toll free path in $\mathcal{G}$ between them, and similarly, a toll free arc from the head of a toll arc to the destination node is added to $\mathcal{A}_{2}^{k}$ if there is a toll free path in $\mathcal{G}$ between them. The cost of a toll free arc $a=(i, j)$ is given by $\gamma_{i, j}^{k}(\infty)$, which is calculated in the original graph. The NPP in the SPGM constructed with these rules is equivalent to the original NPP in the sense that the optimal solution and its value are equal. We can observe that toll free arcs between toll arcs can be computed once for all commodities. Only toll free arcs linking origin and destination nodes to toll arcs need to be calculated separately for each commodity. An example of the SPGM graph for one commodity is depicted in Figure 1.10, where there is one commodity travelling from $o$ to $d$.

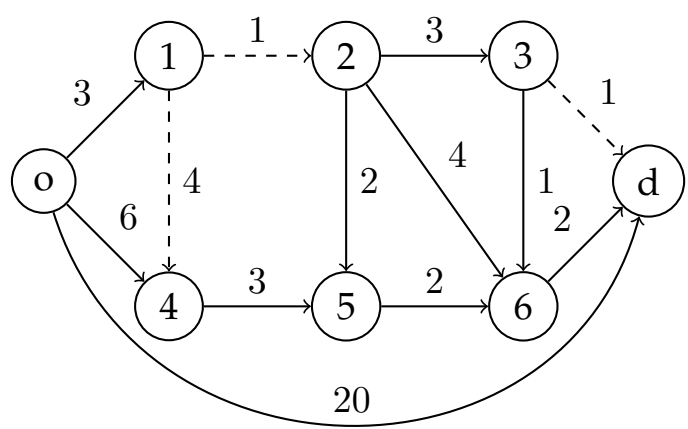

(a) Original graph

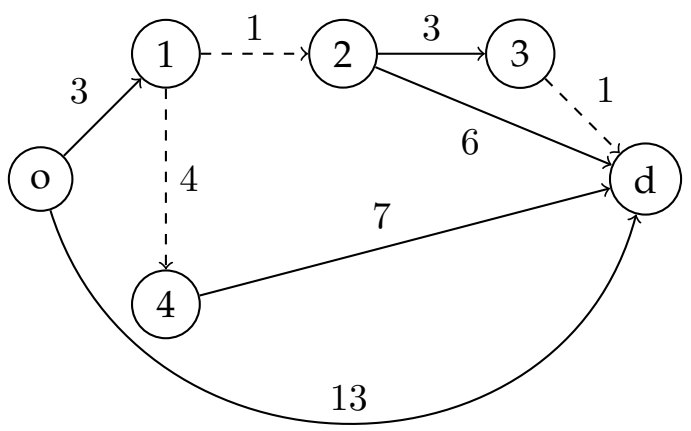

(b) SPGM graph

Figure 1.10: Example of Shortest Path Graph reduction (from Dewez, 2004) 


\subsubsection{Preprocessing}

The SPGM allows us to work on a reduced network for each commodity. It is possible to further reduce the SPGM graph by removing arcs that will not be taken by the commodity regardless of the values of the tolls. Bouhtou et al. (2007b) propose several graph reductions, which are valid for both the SPGM and the original graph. They can therefore also be used as a general preprocessing to reduce the size of the original network. We will only provide definitions and examples of reduction, for the proofs the interested reader is referred to Bouhtou et al. (2007b).

Consider the SPGM with the reduced graph $\mathcal{G}^{k}=\left(\mathcal{N}^{k}, \mathcal{A}_{1}^{k} \cup \mathcal{A}_{2}^{k}\right)$ for one commodity $k \in \mathcal{K}$. For simplicity, in the remainder of this section we will omit the commodity index, as we will always consider networks with only one commodity. Note that $\gamma_{i, j}(\infty)$ is an upper bound and $\gamma_{i, j}(0)$ a lower bound on the cost for the path from $i$ to $j$.

There are six cases in which one or more arcs can be cancelled from a network:

1. if $\gamma_{j, t}(0)=\gamma_{j, t}(\infty)$, then if an optimal path from $s$ to $t$ passes through node $j$, it will use arc $(j, t)$; hence all other arcs leaving $j$ can be removed;

2. if $\gamma_{s, i}(0)=\gamma_{s, i}(\infty)$, then, similarly, any optimal path from $s$ to $t$ using node $i$ will use $\operatorname{arc}(s, i)$ and all other arcs entering $i$ can be removed;

consider now two toll arcs, $\left(i_{1}, j_{1}\right)$ and $\left(i_{2}, j_{2}\right)$ :

3. if $\gamma_{j_{1}, t}(\infty) \leq \gamma_{j_{1}, i_{2}}(\infty)+c_{i_{2}, j_{2}}+\gamma_{j_{2}, t}(0)$, then we can delete arc $\left(j_{1}, i_{2}\right)$;

4. if $\gamma_{s, i_{1}}(\infty) \leq \gamma_{s, i_{2}}(0)+c_{i_{2}, j_{2}}+\gamma_{j_{2}, i_{1}}(\infty)$, then we can delete arc $\left(j_{2}, i_{1}\right)$;

5. if $\gamma_{s, t}(\infty) \leq \gamma_{s, i_{1}}(0)+c_{i_{1}, j_{1}}+\gamma_{j_{1}, t}(0)$, then we can delete toll arc $\left(i_{1}, j_{1}\right)$;

6. if $\gamma_{s, t}(\infty) \leq \gamma_{s, i_{1}}(0)+c_{i_{1}, j_{1}}+\gamma_{j_{1}, i_{2}}(\infty)+c_{i_{2}, j_{2}}+\gamma_{j_{2}, t}(0)$, then we can delete arc $\left(j_{1}, i_{2}\right)$.

In Figure 1.11, we report an example of these reductions, in which, for simplicity, toll arcs have zero fixed cost. For instance, we can remove the toll free arc $(4,1)$ using the fourth reduction: take the two toll arcs $(1,2)$ and $(3,4)$, we have $\gamma_{o, 1}(\infty)=2 \leq$ $4+1=\gamma_{4,1}(\infty)+\gamma_{o, 3}(0)$. Similarly, we can remove arcs $(2,3)$ and $(4,5)$. Moreover, we can remove the toll arc $(5,6)$ using the fifth reduction: $\gamma_{o, d}(\infty)=11 \leq 10+2=$ $\gamma_{o, 5}(0)+\gamma_{6, d}(0)$.

Bouhtou et al. (2007b) also propose methods to eliminate paths which would never be selected whatever the values of the tolls, exploiting the following path dominance criterion. Consider two paths $p$ and $q$ in the SPGM, where $\mathcal{A}_{p}$ and $\mathcal{A}_{q}$ are the set of toll arcs belonging to $p$ and $q$ respectively, and $c_{p}$ and $c_{q}$ are the sum of fixed cost of toll and toll free arcs belonging to $p$ and $q$ respectively. If $\mathcal{A}_{p} \subseteq \mathcal{A}_{q}$ and $c_{p} \leq c_{q}$, then path $q$ is not cheaper than path $p$ for all toll values. In such a case we say that path $q$ is dominated by path $p$. An arc that belongs only to dominated paths can be removed 


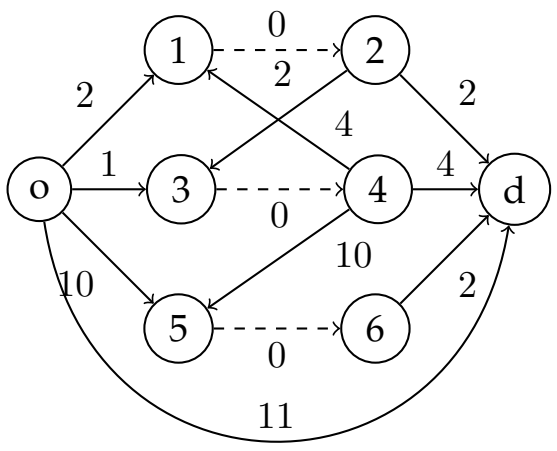

(a) Original graph

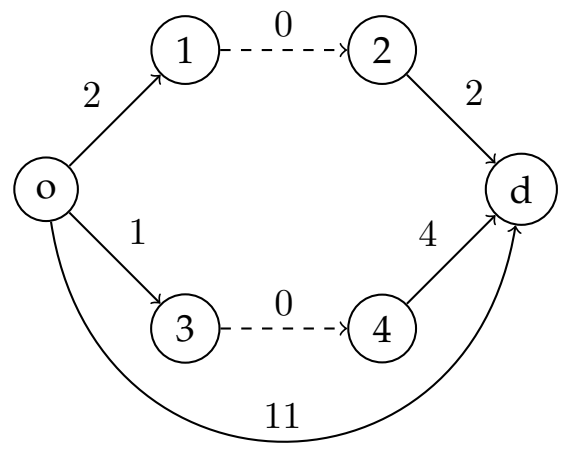

(b) Graph after the arc reduction

Figure 1.11: Example of arc reduction (from Bouhtou et al. 2007b)

from the network without loss of solutions. This is valid also for the toll free path, which can be supposed having an empty set of toll arcs: all toll paths with fixed cost greater than the toll free path cost are dominated. In Figure 1.12(a) path o-1-2-3-4-d is dominated by path o-1-2-d, and so arc 2- 3 can be eliminated from the graph (Figure 1.12(b)).

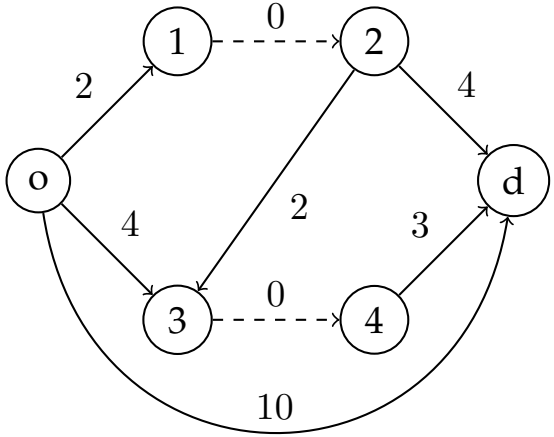

(a) Original graph

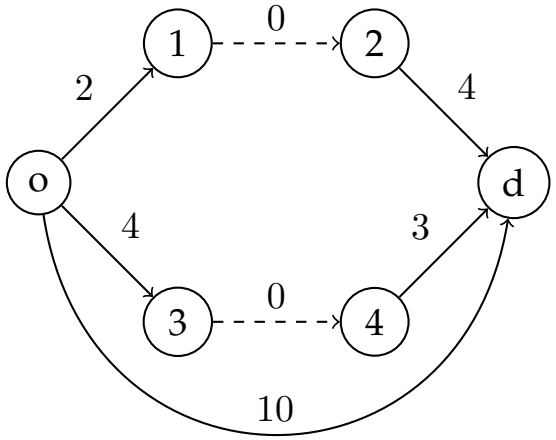

(b) Graph with no dominated paths

Figure 1.12: Example of dominated paths reduction

The number of non-dominated paths for each commodity in a network is bounded by the number of possible subsets of the toll arcs set $\mathcal{A}_{2}^{k}$, i.e. by $2^{\left|\mathcal{A}_{2}^{k}\right|}$, where $\left|\mathcal{A}_{2}^{k}\right|$ is the number of toll arcs in the network for the commodity. In fact if two paths have the same set of toll arcs, then the non-dominated path is the one with the smallest fixed cost, so for each subset of toll arcs there is at most one non-dominated path.

Bouhtou et al. (2007b) show that it exists a network in which this bound is reached. Take a single commodity network with $\left|\mathcal{A}_{2}\right|$ toll arcs, denoted by $a_{1}, \ldots, a_{\left|\mathcal{A}_{2}\right|}$, and a commodity travelling from $o$ to $d$. Toll free arcs are considered as follows. There is a toll free arc from the origin to the tail of each toll arc $a_{i}$ with cost of $3^{i}$, and a toll free arc 
from the head of each toll arc $a_{i}$ to the destination with cost of $3^{\left|\mathcal{A}_{2}\right|+1-i}$. Furthermore, there is a toll free arc between the head of a toll arc $a_{i}$ and the tail of another toll arc $a_{j}$, for each $i<j$, with cost of $3^{j-i}$. Toll arcs have zero fixed costs. Finally, the toll free path from $o$ to $d$ has a cost of $3^{\left|\mathcal{A}_{2}\right|+1}$. In Figure 1.13 we report an example of such a network with three toll arcs. In such a network consider three toll $\operatorname{arcs} a_{i}, a_{j}$ and $a_{k}$ such that $1 \leq i<j<k \leq\left|\mathcal{A}_{2}\right|$. Call $p$ the direct path using toll free arcs from head of $a_{i}$ to tail of $a_{k}$, it has a fixed cost cost $c_{p}=3^{k-i}$ and an empty set of toll arcs, $\mathcal{A}_{p}=\emptyset$. Call $q$ the path going from the head of $a_{i}$ to the tail of $a_{j}$ through toll free arcs, then through toll arc $a_{j}$ and finally from the head of $a_{j}$ to the tail of $a_{k}$ through toll free arcs again. This path has a fixed cost $c_{q}=3^{j-i}+3^{k-j}$ and a set of toll arcs $\mathcal{A}_{q}=\left\{a_{j}\right\}$. We have $\mathcal{A}_{p} \subset \mathcal{A}_{q}$ but $c_{p}>c_{q}$, so no one of the paths dominates the other. This is valid for any pair of paths $p$ and $q$ where $\mathcal{A}_{p} \subset \mathcal{A}_{q}$. We can see that, for each subset of toll arcs, there is a path using them which is not dominated.

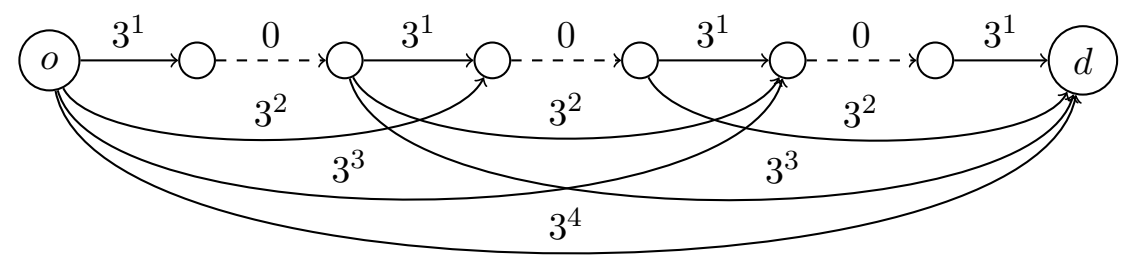

Figure 1.13: Network with an exponential number of undominated paths

A similar result was already showed by Hansen (1980), where the author proved that we can construct a network with $n$ nodes and with $2^{\frac{n-1}{2}}$ non-dominated paths.

Whilst there is no theoretical guarantee of reducing the size of the network with the reduction methods they propose, Bouhtou et al. (2007b) show the effectiveness of their approach and describe a two-phase branch-and-bound algorithm using the SPGM and their preprocessing methods.

\subsubsection{Numerical results}

In Table 1.3 we report numerical results taken from Dewez et al. (2008): the arc pricing problem has been tested on random grid networks involving 60 nodes and 208 arcs, designed to promote interactions between commodities. Fixed costs on arcs are randomly generated in the interval $[2,20]$, for 20, 30 and 40 commodities and 5\%,10\%,15\%,20\% of toll arcs on the total number of arcs in the network. Experiments have been run using CPLEX 8.1 with default values on a Pentium III (500 MHZ). The arc pricing has been tested on the original network (noted as $\mathrm{G}$ in the table) and on the reduced network with SPGM and reductions previously described in Sections 1.4.6 and 1.4.7 (noted as SPGM in the table). The "big M and N" have been first set to arbitrarily high values (BM in the table), and then to the sharp values described in Section 1.4.5 (SM in the table). Moreover some families of valid inequalities (see Dewez et al., 2008) have been 
added to strengthen the formulation ( $+\mathrm{C}$ in the table).

The gap represents the difference in percent between the optimal integer solution value $Z_{\text {opt }}$ and the linear relaxation optimal solution value $Z_{l p}$ : gap $=\left(Z_{l p}-Z_{\text {opt }}\right) / Z_{\text {opt }}$. The "cpu" row gives the cpu times in seconds to solve the problem and the "nodes" row represents the number of nodes in the branch-and-bound tree.

\begin{tabular}{ll|ccc|ccc|ccc} 
& & \multicolumn{3}{|c|}{20 commodities } & \multicolumn{3}{c|}{30 commodities } & \multicolumn{3}{c}{40 commodities } \\
& & $10 \%$ & $15 \%$ & $20 \%$ & $10 \%$ & $15 \%$ & $20 \%$ & $5 \%$ & $10 \%$ & $15 \%$ \\
\hline Arc(G) & gap & $22.8 \%$ & $16.5 \%$ & $16.8 \%$ & $26.2 \%$ & $20.2 \%$ & $22.4 \%$ & $23 \%$ & $24.5 \%$ & $23.6 \%$ \\
BM & cpu & 23 & 349 & - & 99 & 4182 & - & 23 & 158 & - \\
& nodes & 140 & 5943 & - & 447 & 47522 & - & 48 & 1053 & - \\
\hline Arc(G) & gap & $10.2 \%$ & $9.5 \%$ & $10.4 \%$ & $12 \%$ & $11 \%$ & $13.1 \%$ & $7.6 \%$ & $11.1 \%$ & $13.2 \%$ \\
SM & cpu & 13 & 34 & 221 & 59 & 180 & 720 & 13 & 43 & 1341 \\
& nodes & 33 & 161 & 2119 & 173 & 839 & 4391 & 10 & 61 & 8235 \\
\hline Arc(G) & gap & $10.1 \%$ & $9.3 \%$ & $10 \%$ & $11.9 \%$ & $10.7 \%$ & $12.8 \%$ & $7.6 \%$ & $11 \%$ & $13 \%$ \\
SM + C & cpu & 11 & 39 & 133 & 38 & 171 & 788 & 9 & 40 & 1491 \\
& nodes & 26 & 135 & 687 & 77 & 511 & 3179 & 12 & 53 & 6257 \\
\hline Arc(SPGM) & gap & $22.8 \%$ & $16.5 \%$ & $16.8 \%$ & $26.2 \%$ & $20.2 \%$ & $22.4 \%$ & $23 \%$ & $24.5 \%$ & $23.6 \%$ \\
BM & cpu & $<1$ & 12 & 621 & 2 & 160 & - & $<1$ & 3 & - \\
& nodes & 60 & 1264 & 20193 & 314 & 6550 & - & 27 & 305 & - \\
\hline Arc(SPGM) & gap & $10.2 \%$ & $9.5 \%$ & $10.3 \%$ & $12 \%$ & $11 \%$ & $12.9 \%$ & $7.6 \%$ & $11.1 \%$ & $13.2 \%$ \\
SM & cpu & $<1$ & 4 & 66 & 1 & 18 & 226 & $<1$ & 1 & 73 \\
& nodes & 19 & 199 & 1818 & 75 & 535 & 2493 & 7 & 39 & 2590 \\
\hline Arc(SPGM) & gap & $10.2 \%$ & $9.4 \%$ & $10.2 \%$ & $12 \%$ & $10.7 \%$ & $12.8 \%$ & $7.6 \%$ & $11 \%$ & $13.1 \%$ \\
SM + C & cpu & $<1$ & 6 & 101 & 2 & 26 & 231 & $<1$ & 2 & 156 \\
& nodes & 21 & 110 & 1613 & 58 & 405 & 2563 & 7 & 55 & 3621
\end{tabular}

Table 1.3: Numerical results for arc pricing (from Dewez et al., 2008)

The gaps are similar for the formulation on the original network and the SPGM network, supporting the idea that the reduction of the network does not change the combinatorial complexity very much. However, the SPGM reduces the number of nodes and cpu times. On the contrary, using the sharp values for "big $\mathrm{M}$ and N" reduces the gap by a factor of two, and allows the formulation to find the optimal solution for complex instances. Finally cuts have little impact on the gap and on the time, but allow the reduction of the number of nodes in the branch-and-bound tree, when solving the original formulation.

\subsection{Path pricing}

In contrast to the previous problem, where tolls are set on each arc and the commodity is paying for each toll arc used in its shortest path, we will now consider a problem of path pricing, meaning that tolls are associated to paths. A toll path is defined by the subset of toll arcs contained in it, and we associate a toll directly to the path, i.e. to the 
subset of toll arcs. This path pricing version of the NPP is different from the arc version introduced in Section 1.4 .

In fact consider an authority owning a set $\mathcal{A}_{1}$ of toll arcs on a network $\left(\mathcal{N}, \mathcal{A}_{1} \cup \mathcal{A}_{2}\right)$. Solving the arc NPP provides an optimal toll for each arc of $\mathcal{A}_{1}$. However, the authority may instead decide to set tolls on paths containing toll arcs. Since the authority controls only the toll arcs, setting a price for a path is in fact equivalent to setting a price for using the subset of toll arcs of that path, and all paths containing the same subset of toll arcs should have the same toll. Hence, when several paths contain the same subset of toll arcs, only the path with the smallest fixed cost will be considered for pricing. Further, the authority may decide to price only some subset of arcs, e.g. those constituting a simple path in the network (i.e. visiting each node at most once). This special path NPP may yield an optimal revenue higher than its arc version, as we will see with examples in the following section.

\subsubsection{Path pricing vs arc pricing}

First of all, consider the network reported in Figure 1.2 of Section 1.4 for the arc pricing we saw that the leader's optimal revenue is 15 . Consider now the problem of path pricing: there are four toll paths, plus one toll free path of cost 22 (path A-C-E). The first toll path is A-B-C-D-E, with a fixed cost of 6 . The second one is A-C-D-E, with a fixed cost of 12. The third one is A-B-D-E, with a fixed cost of 11. Finally, the fourth one is A-B-C-E, with a fixed cost of 16 . One can see that the second toll path should not be considered as, whatever toll the leader chooses, this path will always be more expensive than the third toll path (the paths have the same set of toll arcs and the third one has a smaller fixed cost). This is an example of path domination briefly introduced above. In this simple example the leader may choose a toll of 16 for the first toll path (the one with the smallest fixed cost), and a large toll on all other paths, such that the shortest path for the commodity will be the first toll path. For this network, the leader's optimal revenue for the path pricing model is thus larger than the one for the arc pricing model.

Consider now the network in Figure 1.14, where there are three commodities, each of them with unit demand and travelling from $o_{1}$ to $d_{1}$, from $o_{2}$ to $d_{2}$ and from $o_{3}$ to $d_{3}$ respectively. Toll arcs 1-2 and 2-3 are connected as in the case of a highway (more details are reported in Section 1.5.4. Using arc pricing gives an optimal solution value of 10 for the leader's revenue, with a toll of 3 on arc 1-2 and of 2 on arc 2-3. On the other hand, using path pricing yields a leader's revenue of 11 , with a toll of 3 on path $o_{1}-1-2-d_{1}$, a toll of 2 on path $o_{3}-2-3-d_{3}$ and a toll of 6 on path $o_{2}-1-2-3-d_{2}$. Note that the toll on this last path is larger than the sum of the toll on arcs composing it. Moreover, the leader's revenue for the path pricing problem is again larger than for the arc pricing one.

We observe that the path pricing problem always yields a leader's revenue greater 
or equal to the arc pricing problem, regardless of the network we consider.

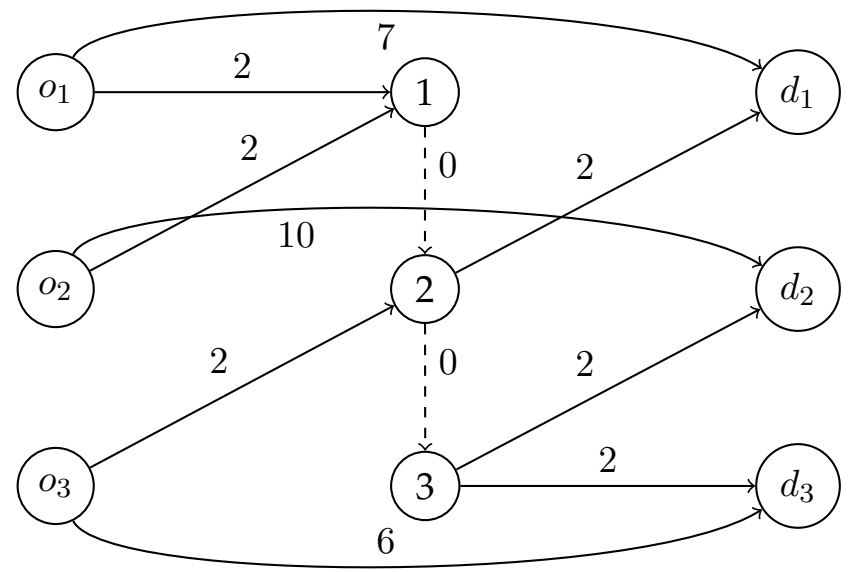

Figure 1.14: Example of arc vs path pricing on a network with connected toll arcs

\subsubsection{Bilevel formulation}

Let us introduce some notation for the path pricing version of NPP. In this case $\mathcal{A}$ represents the set of toll paths whose number is polynomial or fixed. For a general network with $n$ nodes, the maximum number of paths is exponential. For this reason we consider the path pricing problem only for particular situations or networks where the number of toll paths is polynomial or fixed, as we will see in the highway case presented in Section 1.5.4. For each commodity each toll path has a fixed $\operatorname{cost}$ of $c_{a}^{k}$, which is the sum of the fixed costs of the arcs belonging to the path. For each commodity the shortest toll free path is then considered, with cost $c_{o d}^{k}$ and associated binary decision variable $y_{o d}^{k}$. Using this notation, this path NPP can be described as follows:

$$
\begin{aligned}
& \max _{T \geq 0} \sum_{k \in \mathcal{K}} \eta^{k} \sum_{a \in \mathcal{A}} T_{a} x_{a}^{k} \\
& \text { s.t. }(x, y) \in \arg \min _{x, y} \sum_{k \in \mathcal{K}}\left(\sum_{a \in \mathcal{A}}\left(c_{a}^{k}+T_{a}\right) x_{a}^{k}+c_{o d}^{k} y_{o d}^{k}\right) \text {, } \\
& \text { s.t. } \sum_{a \in \mathcal{A}} x_{a}^{k}+y_{o d}^{k}=1 \quad \forall k \in \mathcal{K} \text {, } \\
& x_{a}^{k}, y_{o d}^{k} \in\{0,1\} \quad \forall k \in \mathcal{K}, \forall a \in \mathcal{A},
\end{aligned}
$$

where constraints $(1.11 c)$ allow one path choice for each commodity. In this formulation variables need to be binary (it can be easily seen that this condition could be relaxed for $y$ variables).

The multicommodity path NPP has been proved to be strongly $\mathcal{N} \mathcal{P}$-hard by Heilporn et al. (2010b), whether toll arcs are single or bidirectional, based on a reduction 
from the problem 3-SAT (the proof is similar to the one described in Section 1.4.3 for the arc NPP). The path NPP with only one toll path is equivalent to the single toll arc NPP introduced in Section 1.4.2, and so is polynomial.

\subsubsection{The case of a single commodity}

Contrary to the arc version, the path NPP with only one commodity is polynomial (Dewez, 2004). This problem can be formulated as follows:

$$
\begin{array}{lll}
\max _{x, T} & \eta \sum_{a \in \mathcal{A}} T_{a}, & \\
\text { s.t. } & \sum_{a \in \mathcal{A}} x_{a}=1, & \\
& T_{a} \leq M_{a} x_{a} & \forall a \in \mathcal{A}, \\
& x_{a} \geq 0 & \forall a \in \mathcal{A},
\end{array}
$$

where $M_{a}=\max \left\{0, c_{o d}-c_{a}\right\}$, for all $a \in \mathcal{A}$.

Indeed, the toll path yielding the largest revenue for the leader, i.e. with the largest value of "big M" $\left(\max _{a \in \mathcal{A}} M_{a}\right)$, can be found in polynomial time, searching for the shortest path on the network without any toll. The toll on this path $a$ is set to $M_{a}$ whereas tolls on the other paths are set to sufficiently large values, such that the commodity is not interested in taking them. Notice that this is still valid even if the number of paths to price is not polynomial.

Heilporn et al. (2010b) provide a complete description of the convex hull of solutions in this case. We report more details in Section 1.5.8.

\subsubsection{The NPP with connected toll arcs or Highway Problem}

One widely studied system with a polynomial number of paths is the highway system: it is characterized by a network whose connected toll arcs constitute a single path (as in the network in Figure 1.14). This variant of the NPP has been considered in Heilporn et al. (2010b, 2011). If we make the assumption that users who have left the highway do not re-enter it, paths considered for toll can be uniquely determined by their entry and exit nodes. In consequence, for $n$ nodes in the highway, the number of total paths will be $n^{2}$. Because of the completeness of the toll subgraph, this problem is also called the clique pricing problem.

Figure 1.15 depicts the so-called Complete Toll NPP, introduced in Heilporn et al. (2010b): toll free arcs are inserted between origin and destination nodes, as well as from the origin and destination nodes to the highway, representing shortest toll free paths between the corresponding nodes. Each pair of entry and exit nodes of the highway is linked by a toll subpath. Each toll subpath can be represented by a single artificial toll 
arc (dotted arcs in Figure 1.15). In this a case additivity conditions are not considered, meaning that the toll of a path might not be equal to the sum of tolls on subpaths composing it. For each of these artificial toll arcs, representing toll subpaths $a$, and each commodity $k$ with origin $o^{k}$ and destination $d^{k}$, the fixed cost is denoted as $c_{a}^{k}$ and is calculated as the sum of the cost of the shortest toll free path from $o^{k}$ to the tail of the toll arc $t(a)$, the fixed cost of the toll subpath and the cost of the shortest toll free path from the head of the toll $\operatorname{arc} h(a)$ to $d^{k}$.

Set of entry and exit nodes

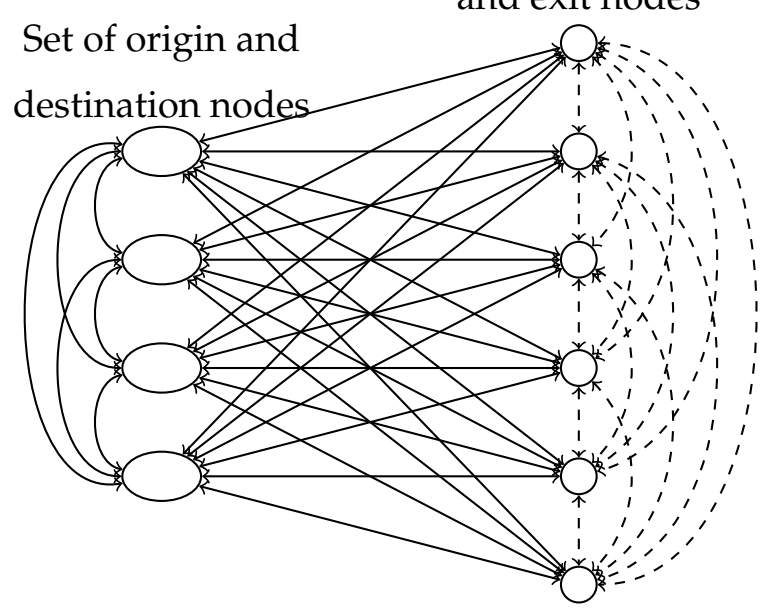

Figure 1.15: Complete toll NPP (from Heilporn et al., 2010b)

Computational experiments revealed that triangle inequalities and monotonicity constraints on the toll variables may not be satisfied by the optimal solution if they are not explicitly included. The guarantee of these conditions is important for real applications, as triangle inequalities prevent a commodity being able to pay less using two (subsequent) highway arcs instead of the direct one from the same origin and destination, and monotonicity constraints imply that the toll of a path cannot be smaller than the toll of any of its subpaths. Mathematically, triangle inequalities are expressed as follows:

$$
T_{a} \leq T_{b}+T_{c} \quad \forall a, b, c \in \mathcal{A}: t(a)=t(b), h(b)=t(c), h(c)=h(a),
$$

whereas monotonicity inequalities are represented by the following conditions:

$$
\begin{aligned}
T_{a} \geq T_{b} & \forall a, b \in \mathcal{A}: t(a)=t(b)<h(a)=h(b)+1 \text { or } t(a)=t(b)-1<h(a)=h(b) \\
\text { or } & t(a)=t(b)>h(a)=h(b)-1 \text { or } t(p)=t(b)+1>h(a)=h(b) .
\end{aligned}
$$

Subnetworks on which these inequalities apply are illustrated in Figure 1.16 .

The highway problem without triangle inequalities and monotonicity constraints can be modelled as the path pricing introduced above (Equations $1.11 \mathrm{a})$ to $(1.11 \mathrm{~d})$ ). 

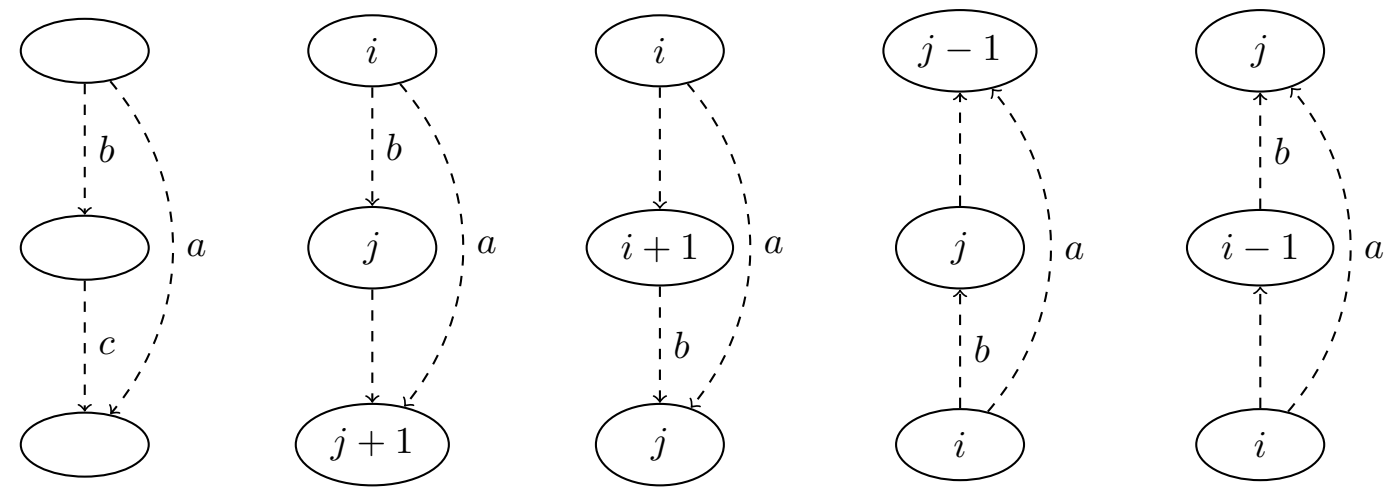

Figure 1.16: Subnetworks on which triangle and monotonicity inequalities apply (from Heilporn et al., 2010b)

Both versions, with and without these constraints, have been proved to be strongly $\mathcal{N} \mathcal{P}$-hard (Heilporn et al., 2010b). We will see from the numerical results in Section 1.5.9 that these constraints make the problem more difficult.

In the following sections we present results for the highway or clique pricing problem which can be extended to the version with triangle inequalities and monotonicity constraints. For this last version further results can be found in Heilporn et al. (2010b, 2011). Then, in the third part of this thesis (Chapters 4 and 5) we provide new formulations and further results on this problem.

\subsubsection{Approximability of the NPP with connected toll arcs}

In this section we address the complexity of the NPP with connected toll arcs or Highway Problem and strengthen previous results proving that it is $\mathcal{A P} \mathcal{X}$-hard. This result excludes the existence of a polynomial time approximation scheme, unless $\mathcal{P}=\mathcal{N} \mathcal{P}$.

Proposition 1.5.1 $(\mathcal{A P} \mathcal{X}$-hardness of $(\mathrm{HP}))$. The Highway Problem $(H P)$ is $\mathcal{A P} \mathcal{X}$-hard, even when each commodity can access the highway from at most two entries and the fixed cost on toll free arcs is restricted to be 0,1 or 2 .

Proof. We consider a reduction from MAX-2-SAT-3 (see for example Garey and Johnson, 1979) to the (HP), similarly as done by Bouhtou et al. (2007a) to prove the $\mathcal{A P X}$ hardness of the problem of pricing bridges over a river.

Consider a 2-SAT-3 formula $F=\bigwedge_{i=1}^{K}\left(l_{i, 1} \vee l_{i, 2}\right)$, with $k$ clauses and two literals per clause, $l_{i, j}$ for $j=1,2$. Each literal corresponds to a variable $x_{1}, \ldots, x_{n}$ or to its negation. Each variable occurs in at most 3 clauses. The question of a MAX-2-SAT-3 problem is to find a truth assignment that satisfies the maximum number of clauses.

As done by Heilporn et al. (2010b) in the $\mathcal{N} \mathcal{P}$-hardness proof for $(\mathrm{HP})$, each variable $x_{i}, i \in\{1, \ldots, n\}$ is represented as a subnetwork, where there are two connected toll arcs $a_{i}$ and $\overline{a_{i}}$, representing the variable $x_{i}$ and its negation $\overline{x_{i}}$ respectively. There are then three commodities, with origin and destination $\left(o^{i 1}, d^{i 1}\right),\left(o^{i 2}, d^{i 2}\right)$ and $\left(o^{i 3}, d^{i 3}\right)$, respectively, and unit demand. The first commodity can pass only through the toll arc 
$a_{i}$ or a toll free path of cost 2. Similarly, the third commodity can pass only through the toll arc $\overline{a_{i}}$ or a toll free path of cost 2 . The second commodity can pass through either one of the two toll arcs or both of them, or a toll free path of cost 1 . This subnetwork is represented in Figure 1.17. We can see that this subnetwork gives a maximal revenue of 4 , with a toll of 2 on one toll arc and of 1 on the other toll arc, and routing the first commodity through toll arc $a_{i}$, the third commodity through toll arc $\overline{a_{i}}$ and the second commodity through the toll arc with toll of 1 .

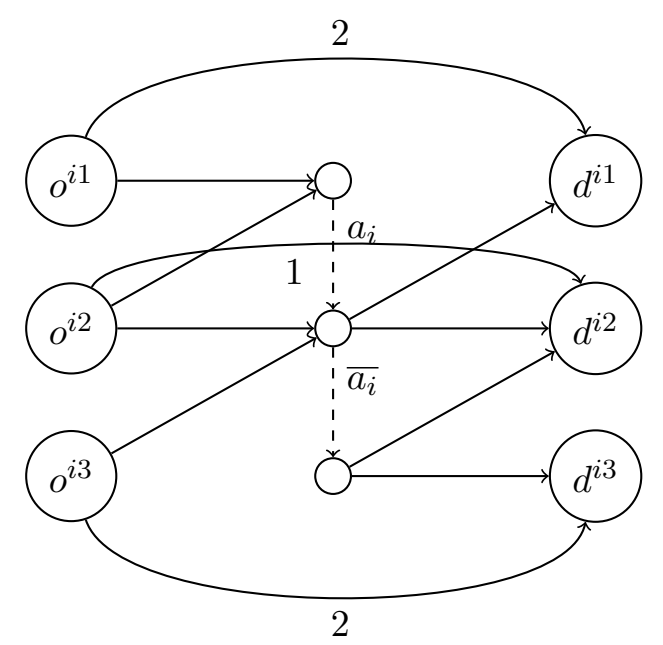

Figure 1.17: $\mathcal{A P} \mathcal{X}$-hard proof for (HP): subnetwork corresponding to variable $x_{i}$

Subnetworks are then linked together, such that the highway corresponds to the connected path of toll arcs (see Figure 1.18).

Furthermore, for each clause $k$, we add a clause-commodity with origin $o^{k}$, destination $d^{k}$ and unit demand. These nodes are connected to the highway such that the commodity can pass through the toll arcs corresponding to the variables (positive or negative) that appear into the clause. Each clause-commodity has a toll free path of cost 1 . Each clause-commodity is linked to exactly two entry nodes of the highway (2SAT) and at most three clause-commodities are linked to each variable (or pair of toll arcs) (SAT-3). Figure 1.18 represents nodes and arcs for two clause-commodities.

When costs on arcs are not explicitly indicated, they are zero. All toll arcs have zero fixed cost. Arcs not represented in the network do not exist, i.e. they have a very high fixed cost.

The so-defined (HP) instance has $3 n+k$ commodities, $2 n$ toll arcs and $11 n+5 k$ fixed cost arcs, hence the reduction is polynomial.

We claim that the 2-SAT-3 formula has a maximum number of true clauses equal to $r$ if and only if the corresponding (HP) instance has an optimal solution of $4 n+r$ as revenue.

First, consider a truth assignment of $x_{i}$ variables that satisfies $r$ clauses of the MAX2-SAT-3. We fix the toll on arc $a_{i}$ (resp. $\overline{a_{i}}$ ) to 1 and the toll on arc $\overline{a_{i}}$ (resp. $a_{i}$ ) to 2 , if $x_{i}$ is true (resp. if $x_{i}$ is false). We observe that each subnetwork gives a maximal revenue 


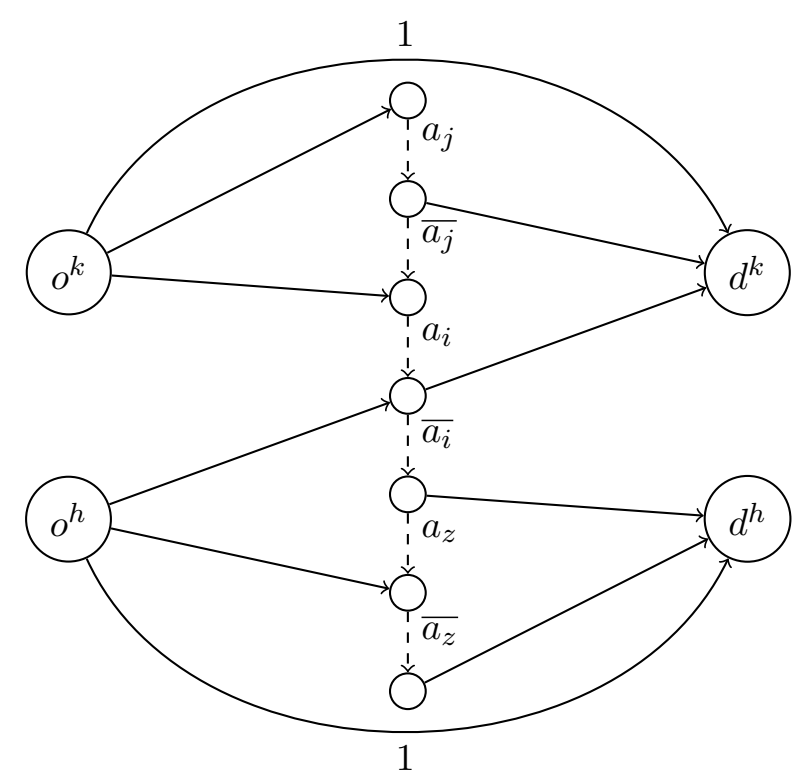

Figure 1.18: $\mathcal{A P} \mathcal{X}$-hard proof for (HP): subnetwork for clause-commodities $\left(x_{j} \vee x_{i}\right) \wedge\left(\overline{x_{i}} \vee \overline{x_{z}}\right)$

of 4 and each satisfied clause-commodity gives an additional revenue of 1 (by routing it through the toll arc with toll of one). Therefore, the optimal revenue for the (HP) is $4 n+r$.

Second, consider an optimal solution for the (HP). Indeed, it makes sense to consider only toll values of 1 or 2 . Now, consider the two toll $\operatorname{arcs} a_{i}$ and $\overline{a_{i}}$ associated to a variable and its negation: in the optimal solution, one has a toll of 1 and the other one of 2 . If both tolls are equal to 2 , we can decrease one toll to 1 without lowering the revenue (we loose one from either the first or third commodity associated to this variable, and we gain one from the second commodity). Then, if both tolls are equal to 1 we note that there is one toll arc (either $a_{i}$ or $\overline{a_{i}}$ ) through which at most one clause-commodity is routed (as each variable occurs in at most three clauses). When can raise the toll associated to this toll arc to 2 and compensate the loss in revenue from the clause-commodity by the corresponding commodity in the subnetwork (first or third one). We can conclude that the optimal solution of the (HP) has, for each variable, a toll arc with toll 2 and a toll arc with toll 1 . Moreover this solution defines a valid truth assignment, taking as truth the variables corresponding to toll arcs with toll of 1 (i.e. $x_{i}$ is true if toll on $a_{i}$ is 1 , false if is 2). The optimal revenue of the (HP) is at most $4 n+r$, where $r$ denotes the number of satisfied clause-commodities.

\subsubsection{One level non-linear formulation}

The path pricing problem (Equations 1.11a to 1.11d) can be reformulated as a single level optimisation problem. First, the follower's objective function can be separated 
for each commodity, and the lower level optimisation problem can be replaced by constraints stating explicitly that the used path is the shortest one (Equations $1.15 \mathrm{~b}$ ) and (1.15c). Moreover, variables $y_{o d}^{k}$, associated to toll free paths, can be eliminated using constraints 1.11c. We introduce a new set of variables $p_{a}^{k}=x_{a}^{k} T_{a}$, having only one non linear set of constraints in the model (1.15e). The non-linear (HP) can be written as follows (Heilporn et al., 2010b):

(HPNL)

$$
\begin{array}{llr}
\max _{T, x, p} & \sum_{a \in \mathcal{A}} \sum_{k \in \mathcal{K}} \eta^{k} p_{a}^{k}, & \\
\text { s.t. } & \sum_{a \in \mathcal{A}}\left[\left(c_{a}^{k}-c_{o d}^{k}\right) x_{a}^{k}+p_{a}^{k}\right]-T_{b} \leq c_{b}^{k}-c_{o d}^{k} & \forall k \in \mathcal{K}, \forall b \in \mathcal{A}, \\
& \sum_{a \in \mathcal{A}}\left[\left(c_{a}^{k}-c_{o d}^{k}\right) x_{a}^{k}+p_{a}^{k}\right] \leq 0 & \forall k \in \mathcal{K}, \\
& \sum_{a \in \mathcal{A}} x_{a}^{k} \leq 1 & \forall k \in \mathcal{K}, \\
& p_{a}^{k}=T_{a} x_{a}^{k} & \forall k \in \mathcal{K}, \forall a \in \mathcal{A}, \\
& x_{a}^{k} \in\{0,1\} & \forall k \in \mathcal{K}, \forall a \in \mathcal{A}, \\
& T_{a} \geq 0 & \forall a \in \mathcal{A} .
\end{array}
$$

We can notice that, if all parameters are integer, variables $T_{a}$ are integer as well, leading to a full integer formulation. A more detailed discussion on this will be provided in Chapter 5, Section 5.3

\subsubsection{One level MILP formulation}

This (HPNL) model can be linearised eliminating the non linear set of constraints (1.15e), introducing the following set of constraints, $\forall k \in \mathcal{K}$ and $\forall a \in \mathcal{A}$ :

$$
\begin{aligned}
& p_{a}^{k} \leq M_{a}^{k} x_{a}^{k} \\
& T_{a}-p_{a}^{k} \leq N_{a}\left(1-x_{a}^{k}\right) \\
& p_{a}^{k} \leq T_{a}
\end{aligned}
$$

The mixed integer linear model is therefore (Heilporn et al., 2010b): 
(HPL)

$$
\begin{array}{llr}
\max _{T, x, p} & \sum_{a \in \mathcal{A}} \sum_{k \in \mathcal{K}} \eta^{k} p_{a}^{k}, & \\
\text { s.t. } & \sum_{a \in \mathcal{A}}\left[\left(c_{a}^{k}-c_{o d}^{k}\right) x_{a}^{k}+p_{a}^{k}\right]-T_{b} \leq c_{b}^{k}-c_{o d}^{k} & \forall k \in \mathcal{K}, \forall b \in \mathcal{A}, \\
& \sum_{a \in \mathcal{A}}\left[\left(c_{a}^{k}-c_{o d}^{k}\right) x_{a}^{k}+p_{a}^{k}\right] \leq 0 & \forall k \in \mathcal{K}, \\
& \sum_{a \in \mathcal{A}} x_{a}^{k} \leq 1 & \forall k \in \mathcal{K}, \\
& p_{a}^{k} \leq M_{a}^{k} x_{a}^{k} & \\
& T_{a}-p_{a}^{k} \leq N_{a}\left(1-x_{a}^{k}\right) & \forall k \in \mathcal{K}, \forall a \in \mathcal{A}, \\
& p_{a}^{k} \leq T_{a} & \forall k \in \mathcal{K}, \forall a \in \mathcal{A}, \forall a \in \mathcal{A}, \\
& p_{a}^{k} \geq 0 & \forall k \in \mathcal{K}, \forall a \in \mathcal{A}, \\
& x_{a}^{k} \in\{0,1\} & \forall k \in \mathcal{K}, \forall a \in \mathcal{A} .
\end{array}
$$

Similarly to arc pricing, constants $M_{a}^{k}$ represent upper bounds on $p_{a}^{k}$ variables, i.e. the highest value that commodity $k$ is willing to pay for path $a$. Therefore, a valid value is $M_{a}^{k}=\max \left\{0, c_{o d}^{k}-c_{a}^{k}\right\}, \forall k \in \mathcal{K}$ and $\forall a \in \mathcal{A}$. Constants $N_{a}$ represent upper bounds on the cost of a path for all commodities, and a valid value is $N_{a}=\max _{k \in \mathcal{K}: a \in \mathcal{A}} M_{a}^{k}$, $\forall a \in \mathcal{A}$.

\subsubsection{Valid inequalities}

We now describe a family of valid inequalities that amounts to a strengthening of constraints $1.17 \mathrm{~b}$ ) and has been introduced by Heilporn et al. (2010b). They are called "Strengthened Shortest Path Inequalities" (SSPI) and, $\forall k \in \mathcal{K}, \forall b \in \mathcal{A}$, they can be written as:

$$
\sum_{a \in \mathcal{A}}\left(c_{a}^{k} x_{a}^{k}+p_{a}^{k}\right)+c_{o d}^{k}\left(1-\sum_{a \in \mathcal{A}} x_{a}^{k}\right) \leq c_{b}^{k}+T_{b}+\sum_{a \in \mathcal{A} \backslash(\mathcal{S} \cup\{b\})}\left(p_{a}^{k}+\left(c_{a}^{k}-c_{b}^{k}\right) x_{a}^{k}\right)
$$

where $\mathcal{S}$ is any subset of $\mathcal{A}$ (possibly the empty set).

To prove the validity we need to consider four possible cases:

1. If $x_{a}^{k}=0, \forall a \in \mathcal{A}$, then $p_{a}^{k}=0, \forall a \in \mathcal{A}$ by $1.17 \mathrm{e}$ and the inequality becomes $c_{o d}^{k} \leq T_{b}+c_{b}^{k}$, which is valid by $1.17 \mathrm{~b}$.

2. If $x_{b}^{k}=1$, then $x_{a}^{k}=0=p_{a}^{k}, \forall a \in \mathcal{A} \backslash\{b\}$ by $1.17 \mathrm{~d}$ ) and 1.17b. The inequality becomes $p_{b}^{k} \leq T_{b}$, which is valid by $1.17 \mathrm{~g}$. 
3. If $x_{a}^{k}=1$ for $a \in \mathcal{A} \backslash(\mathcal{S} \cup\{b\})$, the inequality becomes $0 \leq T_{b}$, which is valid by $1.17 \mathrm{~g}$ ) and $1.17 \mathrm{~h}$.

4. If $x_{a}^{k}=1$ for $a \in \mathcal{S}$, then the inequality becomes $T_{a}^{k}+c_{a}^{k} \leq T_{b}+c_{b}^{k}$. As $x_{a}^{k}=1, p_{a}^{k}=$ $T_{a}$ and the path corresponding to $a$ must be shorter than the path corresponding to $b$, i.e. the inequality is valid.

For any choice of the set $\mathcal{S}$ to be valid, there is an exponential number of inequalities (1.18), but Heilporn et al. (2010b) propose an efficient separation procedure with a complexity of $O(|\mathcal{A}| \log |\mathcal{A}|)$.

We saw in Section 1.5 .3 that the single commodity case is polynomially solvable (Dewez, 2004). Heilporn et al. (2010b) conduct a study of the polyhedral structure for the single commodity case, showing that the formulation reported in Section 1.5 .7 with the Strengthened Shortest Path Inequalities (1.18) completely describes the convex hull of solutions.

In the case of several commodities, Heilporn et al. (2011) exploit interactions between pairs of commodities to propose a further strengthening of the shortest path inequalities, which can be expressed as follows, $\forall k_{1} \in \mathcal{K}, \forall k_{2} \in \mathcal{K}, \forall b \in \mathcal{A}$ and for any subset $\mathcal{S} \subseteq \mathcal{A}$ :

$$
\sum_{a \in \mathcal{A}}\left(c_{a}^{k_{1}} x_{a}^{k_{1}}+p_{a}^{k_{1}}\right)+c_{o d}^{k_{1}}\left(1-\sum_{a \in \mathcal{A}} x_{a}^{k_{1}}\right) \leq c_{b}^{k_{1}}+T_{b}+\sum_{a \in \mathcal{A} \backslash(\mathcal{S} \cup\{b\})}\left(p_{a}^{k_{2}}+\left(c_{a}^{k_{1}}-c_{b}^{k_{1}}\right) x_{a}^{k_{2}}\right)
$$

Furthermore, the following similar inequalities strengthen constraints $(1.17 \mathrm{c}), \forall k_{1} \in$ $\mathcal{K}, \forall k_{2} \in \mathcal{K}$ and for any subset $\mathcal{S} \subseteq \mathcal{A}$ :

$$
\sum_{a \in \mathcal{A}}\left(c_{a}^{k_{1}} x_{a}^{k_{1}}+p_{a}^{k_{1}}\right)+c_{o d}^{k_{1}}\left(1-\sum_{a \in \mathcal{A}} x_{a}^{k_{1}}\right) \leq c_{o d}^{k_{1}}+\sum_{a \in \mathcal{A} \backslash \mathcal{S}}\left(p_{a}^{k_{2}}+\left(c_{a}^{k_{1}}-c_{o d}^{k_{1}}\right) x_{a}^{k_{2}}\right)
$$

These families define facets of the convex hull of feasible solutions for the twocommodity case.

The number of subsets $\mathcal{S}$ is exponential, and hence the number of constraints (1.19) and (1.20). Heilporn et al. (2011) propose a separation procedure to determine the most violated constraint which has a complexity of $O(|\mathcal{K}||\mathcal{A}| \log |\mathcal{A}|)$. We now describe this procedure as it will be used later in Chapter 4

First of all they observe that, when constructing a constraint (1.19) for a fixed pair of commodities $k_{1}$ and $k_{2}$ and a toll path $b$, the right-hand side will be tighter if we choose $a \in \mathcal{A} \backslash(\mathcal{S} \cup\{b\})$ such that $\widetilde{p}_{a}^{k_{2}}+\left(c_{a}^{k_{1}}-c_{b}^{k_{1}}\right) \widetilde{x}_{a}^{k_{2}}<0$, where $\widetilde{p}_{a}^{k_{2}}$ and $\widetilde{x}_{a}^{k_{2}}$ are the current fractional values of corresponding variables. Therefore, if scalars $\left(\widetilde{p}_{a}^{k_{2}}+c_{a}^{k_{1}} \widetilde{x}_{a}^{k_{2}}\right) / \widetilde{x}_{a}^{k_{2}}$ and $c_{b}^{k 1}$ are both sorted in increasing order, it is possible to easily update the optimal set $\mathcal{S}$ when checking each candidate toll path $a$ in the ordered list. The complexity of 
separation procedure is dominated by the sorting operation $(O(|\mathcal{A}| \log |\mathcal{A}|))$, which is done once at the beginning for each commodity $k_{1}$. A similar separation procedure is used for constraints 1.20 , choosing $a \in \mathcal{A} \backslash \mathcal{S}$ such that $\widetilde{p}_{a}^{k_{2}}+\left(c_{a}^{k_{1}}-c_{o d}^{k_{1}}\right) \widetilde{x}_{a}^{k_{2}}<0$.

\subsubsection{Numerical results}

In Table 1.4 we report numerical results taken from Heilporn et al. (2011). The path pricing has been tested on scenarios built around the network topology of the Canadian Highway 10 (autoroute des Cantons de l'Est, Québec). The instances have then been randomly generated: commodities are represented by pairs of cities chosen in a set of 5 to 8 ones ( $v$ cities therefore means $v(v-1)$ commodities), and toll paths by pairs of entry/exit nodes of the highway containing between 10 to 15 nodes (again $n$ nodes means $n(n-1)$ toll paths). Five scenarios have been constructed for each size, with the demand for each city pairs randomly set between 10 and 100. Fixed costs on all arcs are also randomly generated, with the intention to provide realistic situations (for instance roads beside the highway are considered slower, so their fixed costs are multiplied by a factor of 1.5). For each commodity, toll paths with a higher fixed cost than the toll free path are clearly irrelevant, as they will never be the shortest path for any value of the tolls. They can therefore be removed reducing the size of the network. For each class of instances, in the "feasible paths" row of Table 1.4 we report the number of paths per commodity after this reduction, in the form (Min, Max, Average). The problem has been solved for the path formulation presented in Section 1.5.7 (HP in the table), and also with the Strengthened Shortest Path Inequalities (1.18) (added at the root node and whenever they were violated) described in Section 1.5.8 (+ SSPI in the table). Next, triangle inequalities and monotonicity constraints have been added to the formulation (+TMI in the table), with and without the SSPI.

The gap represents the difference in percent between the optimal integer solution value $Z_{o p t}$ and the linear relaxation optimal value $Z_{l p}$ : gap $=\left(Z_{l p}-Z_{o p t}\right) / Z_{\text {opt }}$. The "cpu" row gives the cpu times in seconds and the "nodes" row represents the number of nodes in the branch-and-bound tree. Table 1.4 reports average values for these elements. The computational time upper bound has been set to 5 hours (18000 seconds).

The models have been implemented with Mosel language of Xpress-MP Optimizer version 18, run with default values on a Pentium $4.3 \mathrm{GHz}$ processor with $2 \mathrm{~Gb}$ of RAM and Linux Kernel.

Results show that the shortest path inequalities have a big impact on the gap and on the number of nodes, supporting the intuition of their efficiency as they are facet defining for the single commodity case. For all but the smallest instances, we observe also a sharp drop in the cpu time. The addition of triangle inequalities and monotonicity constraints to the formulation increases the complexity of the problem, and also for this version of the problem the shortest path inequalities prove to be very efficient. 


\begin{tabular}{ll|ccc|cc} 
& & \multicolumn{3}{|c|}{5 cities } & \multicolumn{2}{c}{8 cities } \\
& & 10 nodes & 12 nodes & 15 nodes & 10 nodes & 12 nodes \\
\hline \multirow{2}{*}{ Feasible paths } & $(1,64,22.6)$ & $(1,69,29.7)$ & $(1,140,41.9)$ & $(1,63,17.6)$ & $(1,92,28.3)$ \\
& & & & & & \\
\hline (HP) & gap & $11.85 \%$ & $16.82 \%$ & $13.10 \%$ & $15.98 \%$ & $19.19 \%$ \\
& cpu & 20 & 188 & 405 & 3520 & 5272 \\
& nodes & 1893 & 4067 & 9797 & 159015 & 147793 \\
\hline (HP) & gap & $1.52 \%$ & $1.84 \%$ & $1.86 \%$ & $3.56 \%$ & $1.59 \%$ \\
+ SSPI & cpu & 21 & 52 & 241 & 3038 & 1313 \\
& nodes & 388 & 103 & 719 & 9722 & 3974 \\
\hline (HP) & gap & $18.10 \%$ & $20.13 \%$ & $19.52 \%$ & $30.09 \%$ & $32.04 \%$ \\
+ TMI & cpu & 2 & 18 & 5 & 262 & 947 \\
& nodes & 184 & 1131 & 407 & 27991 & 263875 \\
\hline (HP) & gap & $6.53 \%$ & $3.98 \%$ & $4.55 \%$ & $12.64 \%$ & $9.96 \%$ \\
+ TMI & cpu & 4 & 10 & 8 & 682 & 1099 \\
+ SSPI & nodes & 69 & 127 & 101 & 9205 & 23473
\end{tabular}

Table 1.4: Numerical results for path pricing (from Heilporn et al., 2011).

\subsection{Product pricing}

As introduced in Section 1.3 , the product pricing problem considers a company producing and pricing a set of products (singularly or in bundles) and aiming to maximise its revenue, and customers aiming to maximise their total utility or minimise their costs when buying. This problem, which has been widely studied in economics, includes different versions regarding the objective function, constraints and variables used to model different situations.

We focus on the version in which the customer choice behaviour is based on reservation prices, i.e. the maximum price each customer is willing to pay for each product (see for instance Shioda et al. 2011). A mixed integer formulation for the product pricing problem has been introduced by Heilporn et al. (2010a). We define $\mathcal{K}$ as the set of customers $k$ and $\mathcal{J}$ as the set of products $j$ sold by the company. Product $o d$ is added to this set and represents the substitutable product sold by competitors, as a "toll free" alternative. The demand of customer $k$ is then expressed as $\eta^{k}$, and the reservation price of customer $k$ for product $j$ as $r_{j}^{k}$. Decision variables are $x_{j}^{k}$, equal to 1 if customer $k$ is buying product $j$ and 0 otherwise. The price set by the company on product $j$ is represented by $\pi_{j}$. The product pricing problem can therefore be modelled as follows: 


$$
\begin{array}{llr}
\max _{\pi, x} & \sum_{k \in \mathcal{K}} \sum_{j \in \mathcal{J}} \eta^{k} \pi_{j} x_{j}^{k}, & \\
\text { s.t. } & \sum_{i \in \mathcal{J} \cup\{o d\}}\left(r_{i}^{k}-\pi_{i}\right) x_{i}^{k} \geq r_{j}^{k}-\pi_{j} & \forall k \in \mathcal{K}, \forall j \in \mathcal{J}, \\
& \sum_{j \in \mathcal{J} \cup\{o d\}} x_{j}^{k}=1 & \forall k \in \mathcal{K}, \\
& \pi_{o d}=0, & \\
& \pi_{j} \geq 0 & \forall j \in \mathcal{J}, \\
& x_{j}^{k} \in\{0,1\} & \forall k \in \mathcal{K}, \forall j \in \mathcal{J} \cup\{\text { od }\} .
\end{array}
$$

In this formulation the company is maximising the revenue given by the sold products (Equation (1.21a)). Constraints $1.21 \mathrm{~b}$ ) guarantee that each commodity is buying the product with the greatest difference between the reservation price and the price set by the company. Constraints (1.21c) impose that each commodity is buying one product, and constraint (1.21d) sets a null price for the "toll free" alternative. Finally constraints (1.21e and $1.21 \mathrm{f})$ define that variables for product prices are non negative and that variables for customers' choices of buying are binary.

A parallel between the pricing of products and of paths of a network has been considered by Heilporn et al. (2010a). If we consider the pricing of substitutable products and of paths of a network (such as the highway problem introduced in Section 1.5.4), we can establish a clear parallel: both problems involve revenue maximisation for the company and utility maximisation for the customers or cost minimisation for travellers. More specifically, when modelling these problems, customers correspond to commodities, products to toll paths, reservation prices (the highest price a buyer is willing to pay for a product) to toll windows (the difference between the toll free path cost and the fixed cost for a toll path, as the space left for tolling), prices to tolls, and product assignment variables to path flow variables, respectively for product and network pricing. The assignment of a commodity to a toll free path corresponds to no purchase, which means that the customer is not buying the product (or buying it from a competitor). Constraints $1.21 \mathrm{~b}$ ) ensure that each customer is buying the product with the largest utility, as constraints $1.17 \mathrm{~b})$ guarantee the use of the shortest path for each commodity in the path pricing problem.

The product pricing model (Equations $1.21 \mathrm{a}$ ) to $1.21 \mathrm{f}$ ) is analogous to the path pricing model presented in Section 1.5.6 and can be linearised as described in Section 1.5.7. Valid inequalities introduced in Section 1.5.8 also apply.

Moreover, the triangle and monotonicity constraints introduced for the network problem (see Section 1.5.4) also make sense for product pricing, when products represent different quantities of the same goods. For quantities $X, Y, Z$ such that $X=Y+Z$, market consistency requires that the triangle inequality on prices holds $\left(\pi_{X} \leq \pi_{Y}+\pi_{Z}\right)$, 
and similarly, if $X \leq Y$, one would expect that $\pi_{X} \leq \pi_{Y}$ (monotonicity).

Heilporn et al. (2011) test this formulation on a set of product pricing instances proposed by Shioda et al. (2011). Numerical results show that this model clearly outperforms the formulation proposed by Shioda et al. (2011), with or even without the valid inequalities.

In Chapter 5, Section 5.2, we provide more insights on this product pricing problem. 


\section{Part II}

Some polynomial pricing problems 



\section{Chapter 2}

\section{Pricing Problems with Unit Toll}

\subsection{Introduction}

In this chapter we introduce two particular cases of the Network Pricing Problem. We consider the NPP framework introduced in Section 1.4 , but we restrict the authority owning the toll arcs to use one of the two following tolling schemes:

Constant Tolling: $T_{a}=T, \forall a \in \mathcal{A}_{1}$; this problem is called Constant Toll Network Pricing Problem (CT-NPP);

Proportional Tolling: $T_{a}=\mu_{a} T, \forall a \in \mathcal{A}_{1}$, where $\mu_{a} \in \mathbb{R}$ is a given parameter associated to each toll arc; this problem is called Proportional Toll Network Pricing Problem (PT-NPP).

It is straightforward to see that the CT-NPP is a particular case of the PT-NPP where $\mu_{a}=1, \forall a \in \mathcal{A}_{1}$. Parameters $\mu_{a}$ in PT-NPP could represent the arc length, such that the authority is imposing a per kilometre toll on his/her arcs.

This chapter is organised as follows. First we consider the arc pricing and the path pricing versions, showing that they are equivalent for these problems. We present different formulations, properties and solution algorithms, exploiting the fact that the authority has only one decision variable $T$ to fix. Finally we show that there exists a relation between these unit toll problems and the parametric shortest path problem, making a link between their computational complexity.

\subsection{Arc pricing and path pricing versions}

\subsubsection{Arc pricing}

Using the notation introduced in Section 1.4 the arc pricing version of these problems can be formulated as a bilevel programming problem as follows: 


$$
\begin{aligned}
& \text { (CT-NPP - Arc) } \\
& \max _{T \geq 0} T \sum_{a \in \mathcal{A}_{1}} \sum_{k \in \mathcal{K}} \eta^{k} x_{a}^{k} \\
& \text { s.t. }(x, y) \in \arg \min _{x, y} \sum_{k \in \mathcal{K}}\left(\sum_{a \in \mathcal{A}_{1}}\left(c_{a}+T\right) x_{a}^{k}+\sum_{a \in \mathcal{A}_{2}} c_{a} y_{a}^{k}\right) \text {, } \\
& \text { s.t. } \sum_{a \in i^{+}}\left(x_{a}^{k}+y_{a}^{k}\right)-\sum_{a \in i^{-}}\left(x_{a}^{k}+y_{a}^{k}\right)=b_{i}^{k} \quad \forall k \in \mathcal{K}, \forall i \in \mathcal{N}, \\
& x_{a}^{k}, y_{a}^{k} \geq 0 \\
& \forall k \in \mathcal{K}, \forall a \in \mathcal{A}_{1} \cup \mathcal{A}_{2}, \\
& \text { s.t. }(x, y) \in \arg \min _{x, y} \sum_{k \in \mathcal{K}}\left(\sum_{a \in \mathcal{A}_{1}}\left(c_{a}+\mu_{a} T\right) x_{a}^{k}+\sum_{a \in \mathcal{A}_{2}} c_{a} y_{a}^{k}\right) \text {, } \\
& \text { s.t. } \sum_{a \in i^{+}}\left(x_{a}^{k}+y_{a}^{k}\right)-\sum_{a \in i^{-}}\left(x_{a}^{k}+y_{a}^{k}\right)=b_{i}^{k} \quad \forall k \in \mathcal{K}, \forall i \in \mathcal{N} \text {, } \\
& \max _{T \geq 0} T \sum_{a \in \mathcal{A}_{1}} \mu_{a} \sum_{k \in \mathcal{K}} \eta^{k} x_{a}^{k} \\
& \text { (PT-NPP - Arc) } \\
& x^{k}, y^{k} \geq 0 \\
& x_{a}^{k}, y_{a}^{k} \geq 0 \\
& \forall k \in \mathcal{K}, \forall a \in \mathcal{A}_{1} \cup \mathcal{A}_{2},
\end{aligned}
$$

where $i^{-}$and $i^{+}$denote the sets of arcs with node $i$ as head or tail respectively, and $b_{i}^{k}$ is equal to -1 if $i$ is the origin node of commodity $k, 1$ if it is the destination node, or 0 otherwise. The second level is a shortest path problem for each commodity, whilst at the first level the authority is maximising the revenue from the tolls. A single level MIP formulation can be obtained as detailed in Section 1.4.4 for the general arc version of NPP.

\subsubsection{Path pricing}

In the path pricing version of the NPP tolls are associated directly to paths and not to arcs (for more details we refer to Section 1.5).

Consider the path pricing version on a network with toll and toll free arcs where the authority is imposing tolls on paths. Let us remind that in this case $\mathcal{A}^{k}$ represents the set of toll paths $a$ that the commodity $k$ can use on the network, with $\mathcal{A}^{k} \subseteq \mathcal{A}$, the complete set of toll paths on the network. For each commodity and toll path we 
consider a fixed $\operatorname{cost} c_{a}^{k}$, which is equal to the sum of fixed costs of toll and toll free arcs belonging to it. For the PT-NPP a parameter $\mu_{a}$ is associated to each toll path, equal to the sum of the parameters of its toll arcs. For the CT-NPP we introduce a parameter $\nu_{a}$ for each toll path, which is equal to the number of toll arcs belonging to the path. The path CT-NPP is therefore a particular path PT-NPP with $\mu_{a}=\nu_{a}$ for each toll path $a \in \mathcal{A}$. Finally for each commodity we consider the shortest toll free path of cost $c_{o d}^{k}$ and associate a binary variable $y_{o d}^{k}$ to it.

The path pricing version of CT-NPP and PT-NPP can therefore be described as follows:

$$
\begin{aligned}
& \text { (CT-NPP - Path) } \\
& \max _{T \geq 0} T \sum_{k \in \mathcal{K}} \eta^{k} \sum_{a \in \mathcal{A}^{k}} \nu_{a} x_{a}^{k}, \\
& \text { s.t. }(x, y) \in \arg \min _{x, y} \sum_{k \in \mathcal{K}}\left(\sum_{a \in \mathcal{A}^{k}}\left(c_{a}^{k}+\nu_{a} T\right) x_{a}^{k}+c_{o d}^{k} y_{o d}^{k}\right) \text {, }
\end{aligned}
$$

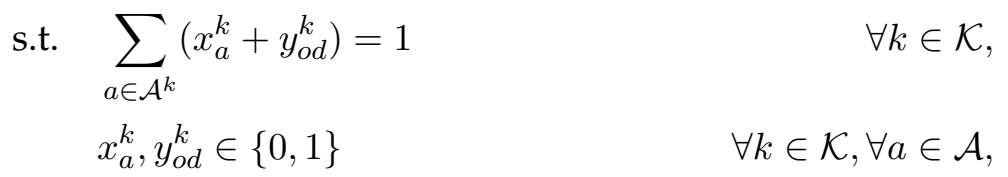

$$
\begin{aligned}
& \max _{T \geq 0} T \sum_{k \in \mathcal{K}} \eta^{k} \sum_{a \in \mathcal{A}^{k}} \mu_{a} x_{a}^{k}, \\
& \text { s.t. }(x, y) \in \arg \min _{x, y} \sum_{k \in \mathcal{K}}\left(\sum_{a \in \mathcal{A}^{k}}\left(c_{a}^{k}+\mu_{a} T\right) x_{a}^{k}+c_{o d}^{k} y_{o d}^{k}\right) \text {, } \\
& \text { s.t. } \quad \sum_{a \in \mathcal{A}^{k}}\left(x_{a}^{k}+y_{o d}^{k}\right)=1 \\
& x_{a}^{k}, y_{o d}^{k} \in\{0,1\} \\
& \forall k \in \mathcal{K},
\end{aligned}
$$

In the second level problem each commodity is looking for its shortest path on the network choosing between toll paths and its toll free path, whilst in the first level the authority is maximising his/her revenues. We can obtain a single level non-linear formulation and a MILP formulation as explained for the general path NPP in Sections 1.5.6 and 1.5 .7 respectively. Note that in these single level formulations the order of magnitude of the number of variables and constraints is the number of commodities multiplied by the number of toll paths. 


\subsubsection{Arc pricing vs path pricing}

As detailed in Section 1.5.1, the general arc and path NPP are different problems and the optimal toll on a path do not necessarily coincide with the sum of optimal tolls of the arcs belonging to the path. We saw that the path NPP may yield an optimal revenue higher than its corresponding arc version.

However, for both the CT-NPP and the PT-NPP, arc and path pricing problems introduced in Sections 2.2.1 and 2.2.2 are equivalent, meaning that they provide the same optimal solution. Denote $T_{C T-a r c}^{*}$ and $T_{P T-a r c}^{*}$ as the optimal solution of the arc pricing versions (Equations (2.1a) to (2.1d) and Equations (2.2a) to (2.2d) for CT-NPP and PT-NPP respectively) and $T_{C T-p a t h}^{*}$ and $T_{P T-p a t h}^{*}$ as the optimal solution of the path pricing versions (Equations (2.3a) to (2.3d) and Equations (2.4a) to (2.4d) respectively.). The following proposition is valid.

Proposition 2.2.1 (Arc pricing is equivalent to path pricing for CT-NPP and PT-NPP). $T_{C T-a r c}^{*}=T_{C T-p a t h}^{*}$ and $T_{P T-a r c}^{*}=T_{P T-p a t h}^{*}$ and the optimal objective function values also coincide.

Proof. This result intuitively follows from the unit toll problem structure: the authority can fix a unique toll on all toll arcs and toll paths. In both arc and path versions commodities are paying tolls on how many toll arcs (in the CT-NPP) or how many units of toll arcs (in the PT-NPP) they are using, such that the toll on a path must be equal to the sum of tolls of arcs belonging to it.

\subsection{Properties and solution algorithm}

\subsubsection{Path dominance criterion}

In Section 1.4 we introduced a concept of path dominance: if two or more paths contain the same subset of toll arcs, only the path with the smallest fixed cost will be considered for pricing by a commodity (it will be cheaper regardless of the toll imposed on the subset of toll arcs). This concept can be extended for the CT-NPP and PT-NPP, using the unit toll imposed by the authority.

Consider first the CT-NPP: for a commodity $k$ each toll path $a$ has a total cost of $c_{a}^{k}+\nu_{a} T$. A toll path $b$ is dominated by a toll path $a$ if $c_{b}^{k} \geq c_{a}^{k}$ and $\nu_{b} \geq \nu_{a}$ with at least one strict inequality, which means that the fixed costs and the number of toll arcs belonging to $b$ are greater than (or equal to) the fixed costs and the number of toll arcs belonging to $a$.

Similarly for the PT-NPP the total cost of a toll path $a$ for commodity $k$ is equal to $c_{a}^{k}+\mu_{a} T$. A toll path $b$ is dominated by a toll path $a$ if $c_{b}^{k} \geq c_{a}^{k}$ and $\mu_{b} \geq \mu_{a}$ with at least one strict inequality. In Figure 2.1 we represent the cost function for three toll paths $\left(a_{1}\right.$, $a_{2}$ and $\left.a_{3}\right)$ for a commodity for PT-NPP: path $a_{1}$ dominates all the others, as its cost is 
the lowest for all values of $T$. In this situation toll paths $a_{2}$ and $a_{3}$ do not need to be considered while solving the problem, as they will never appear in an optimal solution.

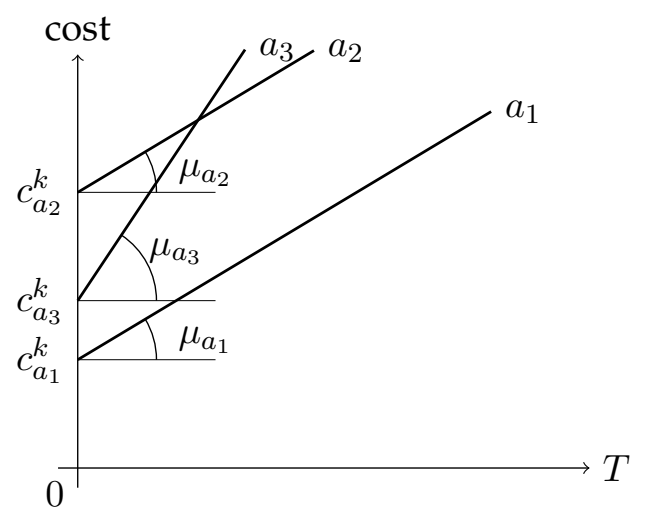

Figure 2.1: Path dominance criterion for PT-NPP

In Section 1.4 .7 we saw that in a network with $\left|\mathcal{A}_{2}\right|$ toll arcs the number of nondominated toll paths for each commodity is bounded by the number of subsets of toll arcs, which is $2^{\left|\mathcal{A}_{2}\right|}$. We also saw with an example that, for the general NPP, this bound can be reached. We can prove a better result for the CT-NPP.

Proposition 2.3.1 (Number of non-dominated paths for CT-NPP). For the CT-NPP, for each commodity $k$ the number of different pairs $\left(c_{a}^{k}, \nu_{a}\right)$ for which there exists a non-dominated toll path $a$ is polynomial and bounded by the number of toll arcs $\left|\mathcal{A}_{2}\right|$ and by $|\mathcal{N}|-1$, where $|\mathcal{N}|$ is number of nodes of the network.

Proof. In the network there are $2^{\left|\mathcal{A}_{2}\right|}$ subsets of toll arcs. We partition them according to their number of toll arcs: we have paths with one toll arc, paths with two toll arcs, and so on, until (possibly) paths with $\min \left\{\left|\mathcal{A}_{2}\right|,(|\mathcal{N}|-1)\right\}$ toll arcs (each path has at most $|\mathcal{N}|-1$ arcs). For each partition set of paths with a certain number of toll arcs $\Upsilon$ and each commodity $k$, we consider the smallest fixed cost $\Gamma$ such that it exists a non-dominated path associated to it with $\left(c_{a}^{k}, \nu_{a}\right)=(\Gamma, \Upsilon)$. We have therefore at most $\min \left\{\left|\mathcal{A}_{2}\right|,(|\mathcal{N}|-1)\right\}$ different pairs $\left(c_{a}^{k}, \nu_{a}\right)$ for which there exists a non-dominated toll path $a$ for each commodity $k$, which is polynomial.

Consider now a PT-NPP where toll arc parameters $\mu_{a}$ are non negative, integer and bounded: $0 \leq \mu_{a} \leq U$ and $\mu_{a} \in \mathbb{N}$, for each $a \in \mathcal{A}_{2}$. This condition is reasonable for practical applications. In such a case we can transform an instance of the PT-NPP to an equivalent instance of the CT-NPP: we replace each $a \in \mathcal{A}_{2}$ by a path of $\operatorname{arcs} b$, with as many arcs $b$ as $\mu_{a}$, each with an unit parameter. The resulting CT-NPP instance has a number of nodes bounded by $U(|\mathcal{N}|-1)$ and a number of toll arcs bounded by $U\left|\mathcal{A}_{2}\right|$, such that this transformation is pseudo-polynomial in the size of the original instance of PT-NPP. For each commodity $k$ the number of pairs $\left(c_{a}^{k}, \mu_{a}\right)$ for which there 
exists a non-dominated toll path $a$ of the PT-NPP instance is therefore bounded by $U \cdot \min \left\{\left|\mathcal{A}_{2}\right|,(|\mathcal{N}|-1)\right\}$ from Proposition 2.3.1.

For the sake of a simpler writing we define a "non-dominated pair" as a pair $\left(c_{a}^{k}, \nu_{a}\right)$ (for the CT-NPP, or replacing $\nu_{a}$ with $\mu_{a}$ for the PT-NPP) for which there exists a nondominated toll path $a$ for commodity $k$.

\subsubsection{Sequential solution procedure}

The CT-NPP and PT-NPP can be solved through a procedure similar to the one described for the case of a single toll arc for the NPP (see Section 1.4.2). These problems have the particularity that the authority is choosing one toll value to impose on his/her arcs. The cost of a toll path therefore depends linearly on this toll value.

Denote the cost for each commodity $k$ and path $a$ as $\operatorname{COST}_{a}^{k}(T)=c_{a}^{k}+\nu_{a} T$ for CT-NPP and $\operatorname{COST} T_{a}^{k}(T)=c_{a}^{k}+\mu_{a} T$ for PT-NPP. Parameters $c_{a}^{k}$ are fixed costs, whilst parameters $\nu_{a}$ and $\mu_{a}$ are variable costs (they are multiplied by the toll). Assume that, for each commodity $k$, we have the set of non-dominated pairs.

Let us consider only the PT-NPP in the remaining of the section, the same results hold for the CT-NPP replacing $\mu_{a}$ with $\nu_{a}$ for each path $a$. As each commodity seeks the shortest path, he/she will take the path of minimum cost depending on the value of $T$. In Figure 2.2(a) we report the cost function of a commodity which can choose from between three toll paths $\left(a_{1}, a_{2}, a_{3}\right)$ and a toll free path (dashed line). Hence, the commodity will choose path $a_{1}$ if the authority imposes a toll in the interval $\left[0, B_{a_{1}}^{k}\right]$, path $a_{2}$ for tolls in $\left[B_{a_{1}}^{k}, B_{a_{2}}^{k}\right]$, path $a_{3}$ for tolls in $\left[B_{a_{2}}^{k}, B_{a_{3}}^{k}\right]$ and the toll free path for all tolls greater than $B_{a_{3}}^{k}$. Values $B_{a}^{k}$ represent "breakpoints" toll values for the commodity's path choice. The cost function of Figure 2.2(a) is a continuous piecewise linear concave function, bounded at the upper limit by the toll free path cost $c_{o d}^{k}$.

The authority is interested only in these breakpoints as possible choices of the toll value, as for any other toll value he/she can increase it (and gain more) without changing the commodity path choice. This reasoning can be repeated for each commodity, finding the total set of breakpoints to be considered by the authority. For each breakpoint (toll value) we calculate the shortest (toll) path for each commodity (defining the values of assignment variables $x_{a}^{k}$ ), and the revenue of the authority is obtained with Equation 2.4a). The authority will choose the toll value corresponding to the breakpoint in which his/her revenue is maximal. The authority's revenue function is reported in Figure 2.2(b) for one commodity and in Figure 2.2(c) for multiple commodities. These functions are non-continuous and linear in each interval of consecutive breakpoint values.

To find the breakpoint values for each commodity $k$ we first order non-dominated pairs in ascending order of fixed costs $c_{a}^{k}$ and in decreasing order of parameters $\mu_{a}$, such that $c_{a_{i}}^{k} \leq c_{a_{i+1}}^{k}$ and $\mu_{a_{i}} \geq \mu_{a_{i+1}}$, for $i=1,2, \ldots,\left|\mathcal{A}^{k}\right|$, where $\left|\mathcal{A}^{k}\right|$ is the number of nondominated pairs for commodity $k$. We set $c_{\left|\mathcal{A}^{k}\right|+1}^{k}=c_{o d}^{k}$ and $\mu_{\left|\mathcal{A}^{k}\right|+1}=0$, corresponding 


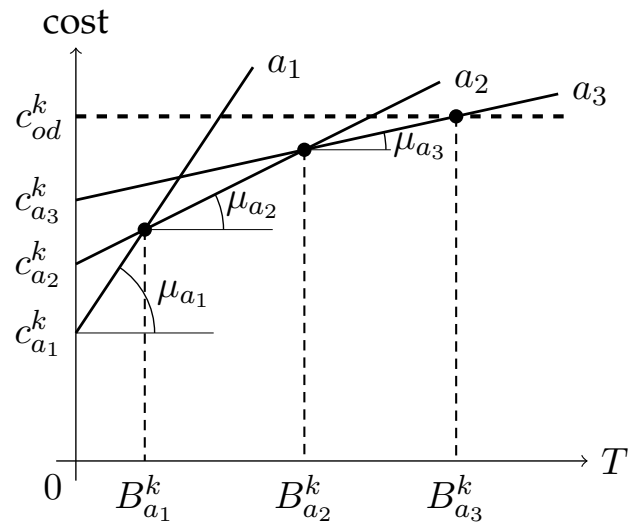

(a) Cost for one commodity

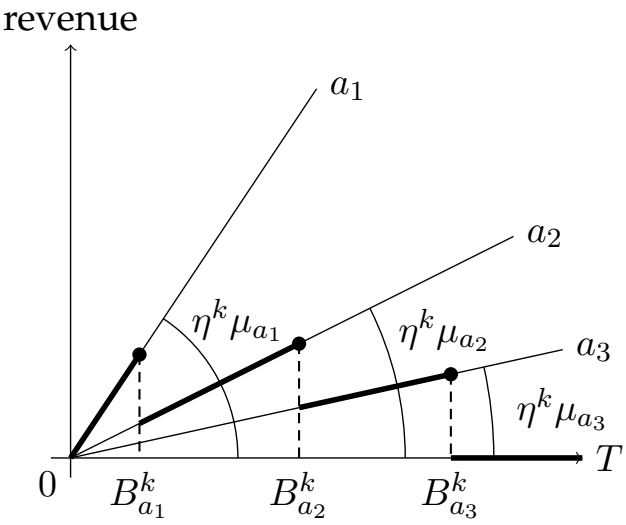

(b) Authority's revenue for one commodity

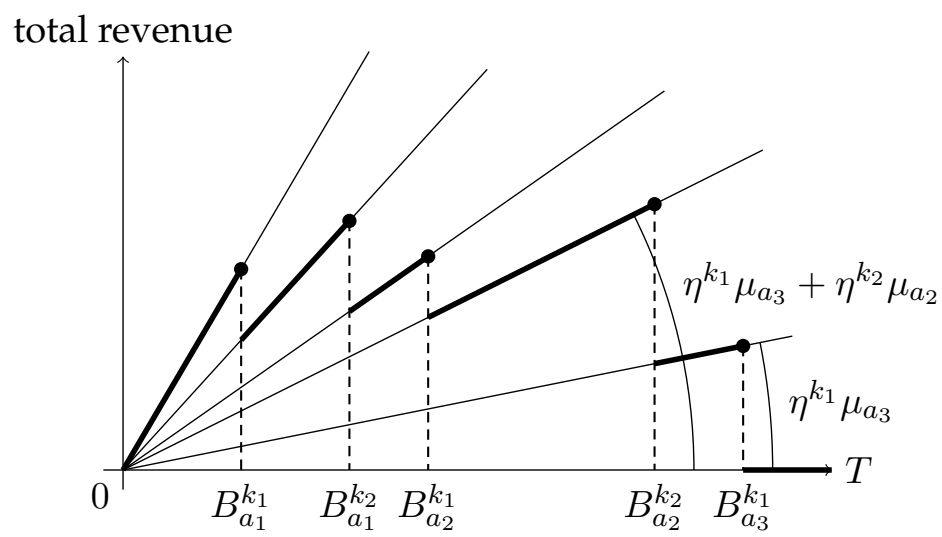

(c) Total authority's revenue

Figure 2.2: Sequential solution procedure for PT-NPP - Functions on $T$

to the toll free path. This ordering is valid due to the path dominance criteria: if $c_{a_{i}}^{k} \leq$ $c_{a_{i+1}}^{k}$ and $\mu_{a_{i}} \leq \mu_{a_{i+1}}$ with at least one strict inequality, then path $a_{i+1}$ is dominated by path $a_{i}$ and will have been eliminated. We obtain breakpoint values with the following equation:

$$
B_{a_{i}}^{k}=\frac{c_{a_{i+1}}^{k}-c_{a_{i}}^{k}}{\mu_{a_{i}}^{k}-\mu_{a_{i+1}}^{k}} \quad \forall k \in \mathcal{K}, i=1,2, \ldots,\left|\mathcal{A}^{k}\right| .
$$

The total number of breakpoints is bounded by $|\mathcal{K}|\left|\mathcal{A}^{k}\right|$. For the CT-NPP this bound is equal to $|\mathcal{K}| \min \left\{\left|\mathcal{A}_{2}\right|,(|\mathcal{N}|-1)\right\}$, whilst for the limited PT-NPP (see Section 2.3.1) it is equal to $U|\mathcal{K}| \min \left\{\left|\mathcal{A}_{2}\right|,(|\mathcal{N}|-1)\right\}$.

We associate to each breakpoint value $B_{a}^{k}$, obtained for commodity $k$ and toll path $a$, a gain $S_{a}^{k}$, which is the amount of toll units the authority will get when choosing this breakpoint as toll value (the revenue for the authority will be $S_{a}^{k} B_{a}^{k}$ ):

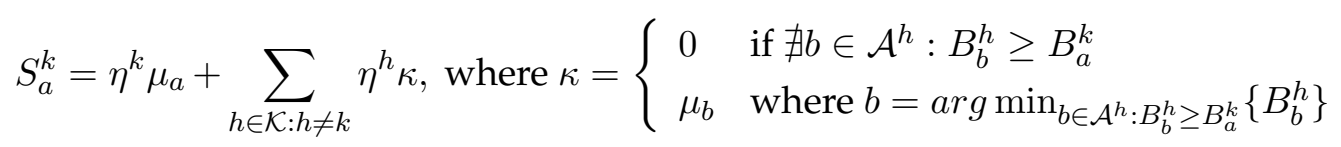


The optimal solution will be $T^{*}=B_{a}^{k}$ where $(a, k)=\arg \max _{k \in \mathcal{K}, a \in \mathcal{A}^{k}}\left\{B_{a}^{k} S_{a}^{k}\right\}$. The following algorithm formalises the solution procedure.

Algorithm 2.3.1 (Sequential solution procedure for CT-NPP and PT-NPP).

Step 1: for each commodity $k \in \mathcal{K}$ :

(a) obtain the set of all non-dominated pairs $\mathcal{A}^{k}$;

(b) order non-dominates pairs in ascending order of fixed costs $c_{a}^{k}$ and in decreasing order of parameters $\mu_{a}$;

(c) identify the breakpoint toll values, as the values of $T$ for which the commodity has convenience in changing its path choice, using Equation (2.5).

Step 2: consider the total set of breakpoints for all commodities and calculate the corresponding gain for the authority with Equation 2.6.

Step 3: take the toll value for which the revenue (equal to the toll multiplied by the gain) is maximised.

The steps of this algorithm are polynomial except for Step 1(a). We saw that the number of non-dominated pairs is polynomial for the CT-NPP, but it is pseudo-polynomial for the PT-NPP. Furthermore, we need an algorithm to find such pairs. We will address these issues in Section 2.4 using the relation between our unit toll problems and the parametric shortest path problem.

This solution procedure has been used in Castelli et al. (2013) where a PT-NPP is solved with a fixed number of toll paths for each commodity, resulting from the particular air traffic management application addressed.

\subsection{3 (Pseudo-)polynomial path formulation}

Using the breakpoints values described in previous section, we can introduce a formulation for the path versions of our unit toll problems, which is polynomial for the CT-NPP and pseudo-polynomial for the limited PT-NPP. This formulation is similar to the one proposed by Dewez (2004) for the single toll arc NPP that we presented in Section 1.4.2. It is also equivalent to the path formulations introduced in Section 2.2.2.

We know that the optimal toll value coincides with one of the breakpoint values. Consider the set of breakpoints $B_{a}^{k}, k \in \mathcal{K}$ and $a \in \mathcal{A}^{k}$, and associate to each of them a binary variable $\omega_{a}^{k}$, which is equal to 1 if the chosen toll value is the breakpoint value $B_{a}^{k}, 0$ otherwise.

The path version of CT-NPP and PT-NPP can be formulated as follows: 


$$
\begin{array}{ll}
\max _{\omega} & \sum_{k \in \mathcal{K}} \sum_{a \in \mathcal{A}^{k}} S_{a}^{k} B_{a}^{k} \omega_{a}^{k}, \\
\text { s.t. } & \sum_{k \in \mathcal{K}} \sum_{a \in \mathcal{A}^{k}} \omega_{a}^{k}=1, \\
& \omega_{a}^{k} \in\{0,1\}
\end{array}
$$

where $S_{a}^{k}$ is calculated with Equation (2.6). It is straightforward to see that binary conditions on variables (constraints (2.7c) can be relaxed, leading to a polynomial formulation for the CT-NPP and a pseudo-polynomial formulation for the PT-NPP, as there are as many variables as non-dominated pairs.

\subsection{Relation with the parametric shortest path problem}

In this section we show that the second level problem solved by each commodity when the toll imposed by the leader varies in the CT-NPP and the PT-NPP is a parametric shortest path problem, such that known algorithms for this problem can be used. We first introduce it with some results from the literature and then consider the relation with our unit toll problems.

\subsubsection{The parametric shortest path problem}

Consider a graph $\mathcal{G}=(\mathcal{N}, \mathcal{A})$, where $\mathcal{N}$ is the set of nodes and $\mathcal{A}$ is the set of arcs. Each arc $a \in \mathcal{A}$ has a cost $c_{a} \in \mathbb{R}$. Define $s \in \mathcal{N}$ as a particular node called source. The single-source shortest path problem is to determine the path of smallest cost from the source to every other node of the graph. This problem has been widely studied in the literature (see for instance Chapter 4 of Ahuja et al., 1993), and is polynomial if we assume that cycles of negative cost do not exist in the graph. It is well known that these shortest paths from a source $s$ represent a tree routed in $s$, called shortest path tree.

Consider now a parametric version of such problem, where the cost of an arc $a \in \mathcal{A}$ has the following form:

$$
\operatorname{COST}_{a}=c_{a}+\mu_{a} T \quad \forall a \in \mathcal{A},
$$

where $c_{a} \in \mathbb{R}$ and $\mu_{a} \in \mathbb{R}$ are the fixed cost and the variable cost associated to the arc $a$ respectively, and $T \in \mathbb{R}$ is a parameter we can choose. Let $\mathcal{A}_{2} \subseteq \mathcal{A}$ be a designed subset of arcs such that $\mu_{a}>0$ if $a \in \mathcal{A}_{2}$ and $\mu_{a}=0$ otherwise.

The Parametric Shortest Path Problem (PSPP) is to determine the path of smallest cost from the source to every other node of the graph, for each value of $T$, and it can be formulated as follows: 


$$
\begin{aligned}
& \text { (PSPP) } \\
& \min _{x} \sum_{a \in \mathcal{A}}\left(c_{a}+\mu_{a} T\right) x_{a}, \\
& \text { s.t. } \sum_{a \in i^{-}} x_{a}-\sum_{a \in i^{+}} x_{a}=b_{i} \quad \forall i \in \mathcal{N} \text {, } \\
& x_{a} \geq 0 \quad \forall a \in \mathcal{A},
\end{aligned}
$$

where $i^{-}$and $i^{+}$denote the sets of arcs with node $i$ as head or tail respectively, and $b_{i}$ is equal to $|\mathcal{N}|-1$ if $i=s$, and to -1 for all other nodes. The solution of this problem provides a tree of shortest paths for each value of $T$.

It is evident that the shortest path tree and the cost of its paths vary depending on the value of $T$. If we consider decreasing values of $T$ from $+\infty$ to $-\infty$ there will be a sequence of shortest path trees. In particular there will be some specific values of $T$ where the shortest path tree changes its structure, which can be seen as breakpoints. We would therefore like to know how many breakpoints exist for a certain graph and have an efficient algorithm to find them.

Karp and Orlin (1981) considered a particular case of this PSPP where $\mu_{a}=1$, $\forall a \in \mathcal{A}_{2}$. They show that for this case the shortest path from $s$ to any other node changes at most $|\mathcal{N}|$ times and that the number of breakpoints is bounded by $|\mathcal{N}|^{2}$. They also provide two algorithms to find the breakpoints, with respective running time of $\mathcal{O}\left(|\mathcal{N}|^{3}\right)$ and $\mathcal{O}(|\mathcal{N}||\mathcal{A}| \log |\mathcal{N}|)$. The first algorithm calculates the optimal solutions simultaneously for all values of $T$, which is not typical in parametric programming algorithms. On the other hand the second algorithm calculates the solution (i.e. the shortest path tree) for one value of $T$ at a time, as $T$ decreases from $+\infty$ to $T^{*}$, where $T^{*}$ is the minimum value of $T$ for which there are no cycles of negative cost in the resulting graph. The algorithm provides as solution a sequence of real numbers $+\infty=T_{0}, T_{1}, \ldots, T_{m}=T^{*}$ (i.e. the breakpoints) and a sequence of trees $Y_{0}, Y_{1}, \ldots, Y_{m}$ such that the tree $Y_{i}$ is the tree of shortest paths for $T \in\left[T_{i}, T_{i+1}\right]$. They assume that there exists a path from $s$ to $v$, for each $v \in \mathcal{N}$, and that there is no cycle of negative cost composed solely of arcs in $\mathcal{A} \backslash \mathcal{A}_{2}$.

Later Young et al. (1991) improved Karp and Orlin's result providing an algorithm running in $\mathcal{O}\left(|\mathcal{N}||\mathcal{A}|+|\mathcal{N}|^{2} \log |\mathcal{N}|\right)$, which is a modification of Karp and Orlin's second algorithm with Fibonacci heap data structures.

Erickson (2010) considers the parametric shortest path as the resulting problem on the dual of a planar graph when solving the maximum flow or minimum cut problems. He considers $\mu_{a}=\{-1,1\}$ for each $a \in \mathcal{A}_{2}$ and shows that the number of breakpoints is still $O\left(|\mathcal{N}|^{2}\right)$ and the algorithms proposed by Karp and Orlin (1981) and Young et al. (1991) can be adapted with trivial modifications. This is also valid when parameters $\mu_{a}$ have only a constant number of different values. 
Consider now the general case where parameters $\mu_{a}$ can be different for each arc $a$ of $\mathcal{A}_{2}$, but constrain them to be non negative, integer and bounded: $0 \leq \mu_{a} \leq U$ and $\mu_{a} \in \mathbb{N}$, for each $a \in \mathcal{A}_{2}$. In such a case we can transform any instance replacing each arc of $\mathcal{A}_{2}$ with a path of arcs with a unit parameter, composed by as many arcs as the $\mu_{a}$ value. The resulting instance is of the type considered by Karp and Orlin (1981) but with a number of arcs of $O\left(U\left|\mathcal{A}_{2}\right|\right)$. Therefore the shortest path from $s$ to any other node changes at most $U|\mathcal{N}|$ times, and the number of breakpoints is $O\left(U|\mathcal{N}|^{2}\right)$, which is pseudo-polynomial in the size of the initial instance.

The general case of the PSPP where $\mu_{a} \in \mathcal{R}$ is approached by Gusfield (1980), where the author proves that the upper bound on the number of breakpoints is $|\mathcal{N}|^{\log |\mathcal{N}|}$. Later Carstensen (1983) and Mulmuley and Shah (2000) shows that there exists a network in which there are $2^{\Omega \log ^{2}|\mathcal{N}|}$ breakpoints. Notice that $|\mathcal{N}|^{\log |\mathcal{N}|}=2^{\log ^{2}|\mathcal{N}|}$.

\subsubsection{Relation between unit toll problems and the parametric shortest path problem}

In the CT-NPP (Equations (2.1a) to (2.1d) ) and the PT-NPP (Equations 2.2a to 2.2d) each commodity is searching for the shortest path between its origin and destination, for each toll value $T \in \mathbb{R}$ the authority is imposing on his/her toll arcs. Therefore it is straightforward to see that the second level problem of these problems is a parametric shortest path problem for each commodity, where the set $\mathcal{A}_{2} \in \mathcal{A}$ corresponds to the set of toll arcs and the set of toll free arcs is $\mathcal{A}_{1}=\mathcal{A} \backslash \mathcal{A}_{2}$. We can use PSPP results and algorithms to find the breakpoint values for the tolls and the optimal solution for the authority will be one of these values. Using PSPP results and algorithms we overcome the problem of finding all pairs of fixed/variable costs for which there exists a nondominated toll path that was left unsolved by the algorithm presented in Section 2.3.2.

Let us make a remark: the PSPP provides as a solution the shortest path between a chosen source node and all other nodes, whilst in our CT-NPP and PT-NPP we consider a commodity as a pair of origin/destination nodes and we are interested in the shortest path between these two nodes. Let us denote as $O(\mathcal{K})($ resp. $D(\mathcal{K}))$ the set of origin (resp. destination) nodes of all commodities. Therefore solving the CT-NPP and PT$\mathrm{NPP}$ with $|\mathcal{K}|$ commodities correspond to solve $\min \{|O(\mathcal{K})|,|D(\mathcal{K})|\}$ PSPPs.

The second level of the CT-NPP is the case considered by Karp and Orlin (1981) with $\mu_{a}=1$ for all $a \in \mathcal{A}_{2}$, such that the following proposition holds.

Proposition 2.4.1 (CT-NPP is polynomial). A multi commodity instance of CT-NPP can be solved in $\mathcal{O}(\min \{|O(\mathcal{K})|,|D(\mathcal{K})|\}|\mathcal{N}||\mathcal{A}| \log |\mathcal{N}|)$, which is polynomial.

Proof. We use Karp and Orlin's second algorithm introduced above to solve a PSPP for each commodity (second level problem) and find the set of breakpoints. We therefore need to solve $\min \{|O(\mathcal{K})|,|D(\mathcal{K})|\}$ problems, each of $\mathcal{O}(|\mathcal{N}||\mathcal{A}| \log |\mathcal{N}|)$. Call $B$ the total set of breakpoints for all commodities. There are at most $\min \{|O(\mathcal{K})|,|D(\mathcal{K})|\}|\mathcal{N}|^{2}$ 
breakpoints. The algorithm provides the corresponding shortest path tree for each toll value (breakpoint or not), such that we know the path used by each commodity and the consequent authority's revenue for each breakpoint of $B$. The optimal toll corresponds to the breakpoint maximising the revenue.

If as introduced in Section 2.3.1 we limit the parameter associated to toll arcs to be non negative, integer and bounded, the second level of the PT-NPP is a PSPP with $0 \leq \mu_{a} \leq U$ and $\mu_{a} \in \mathbb{N}$, for each $a \in \mathcal{A}_{2}$. Karp and Orlin's second algorithm can be used to solve the problem, and the total number of breakpoints will be bounded by $U \cdot \min \{|O(\mathcal{K})|,|D(\mathcal{K})|\}|\mathcal{N}|^{2}$, which is pseudo-polynomial in the size of the instance. 


\section{Chapter 3}

\section{Robust Version of Some Polynomial Pricing Problems}

\subsection{Introduction}

In all the other chapters of this thesis we suppose that the parameters of our problems, i.e. the values that define an instance such as the arc/path costs and the commodities demand, are precisely known and equal to some nominal values. However, in real applications, parameters may take values different from the nominal ones, such that the optimal solution found with the nominal values is no longer optimal or even feasible. Therefore we would like to design a solution approach that addresses parameters uncertainty, being "robust" from this point of view.

Robustness for optimisation problems has been widely studied in the literature, both for linear and combinatorial problems. To the best of our knowledge, until now there is no study considering a robust approach for the Network Pricing Problem, which is the object of interest of this thesis. In this chapter we would like to propose a first attempt to deal with robustness for the NPP, starting from some "easy" cases.

We introduce uncertainty on the parameters of some (pseudo)-polynomial NPPs, such as the single toll arc case (see Section 1.4.2), the single commodity case for the path version (see Section 1.5.3 and the unit toll case (see Chapter 2). Uncertainty is expressed by intervals to which unknown parameters are supposed to belong, and solutions are sought for the worst case scenario. We analyse if the robust version of each problem is more difficult than its deterministic counterpart and we propose algorithms to find optimal robust solutions.

This chapter is organised as follows. First of all we present a brief description of robust optimisation. This description does not want to be exhaustive, we synthetically present what is needed to understand the second part of the chapter and we refer the reader to the literature for further details. In the second part of the chapter we address the different pricing problems, define their robust counterparts, and present theoretical results together with solution algorithms. 


\subsection{A brief description of robustness}

A robust solution to an optimisation problem is a solution which is still feasible and "nearly" optimal if its parameters vary in a certain range. The problem solution must therefore be robust against a certain variation of its parameters value, and we would like to have an algorithm to find it which is as efficient as possible.

First of all we distinguish between the two most common representations of uncertainty: probabilistic or non probabilistic. In the probabilistic representation, instead of knowing the nominal value of the parameters, a probabilistic distribution is known for them. This approach, usually called stochastic, has been introduced already by Dantzig (1955) and has been widely studied in stochastic optimisation. References on this topic can be found for instance in the book by Birge and Louveaux (2011).

The non probabilistic representation of uncertainty often considers a set of possible realisations for the parameters (set-based representation of uncertainty) and searches for a solution which is feasible for any realisation of the parameters in the given sets. This approach is usually called robust. The robust approach has been introduced in the early 70s, when Soyster (1973) proposed a linear optimisation model that finds a feasible solution for all parameter values belonging to a convex set. These solutions however are often too conservative, and later effort has been made to address the overconservatism issue, in both linear and discrete programming. A comprehensive review on robust optimisation can be found in Bertsimas et al. (2011), where authors survey both theoretical and applied results, highlighting the modelling power and broad applicability of robust optimisation. More details can be found in the book by Ben-Tal et al. (2009).

Kouvelis and $\mathrm{Yu}$ (1997) study robust discrete optimisation problems and propose a framework which seeks for a solution that optimises the worst case performance under a set of scenarios for the parameters. However, under this approach, the robust counterpart of many polynomially solvable discrete problems becomes $\mathcal{N} \mathcal{P}$-hard. Another approach is the "minimax-regret" which seeks for a solution that minimises the worst case loss that may occur in the objective value. Also in this robust framework many of the polynomially solvable discrete problems become $\mathcal{N} \mathcal{P}$-hard (see for instance Averbakh, 2001).

Bertsimas and Sim (2003, 2004) propose an approach to address parameters uncertainty for mixed-integer optimisation problems that allows to control the degree of conservatism of the solution and that is computationally tractable. The degree of conservatism is expressed in terms of the probabilistic bound of constraint violation. The trade-off between feasibility, optimality and robustness is called the "price of robustness" (Bertsimas and Sim, 2004). We now present this approach in details, since our NPP robustness analysis is based on it.

Bertsimas and Sim (2003, 2004) propose a robust integer programming problem of moderately larger size (with respect to the determinist version) when parameters of 
both the objective function and the constraints of an integer programming problem are subject to uncertainty. They consider a mixed-integer deterministic problem of the following form:

$$
\begin{array}{ll}
\max _{x} & c^{T} x, \\
\text { s.t. } & A x \leq b, \\
& x_{j} \in \mathbb{Z} \quad j=1, \ldots, k,
\end{array}
$$

where $c \in \mathbb{Z}^{n}, A \in \mathbb{Z}^{m \times n}$ and $b \in \mathbb{Z}^{m}$, and the first $k$ variables $x$ are integer.

Parameters uncertainty is defined with intervals, as follows:

for the matrix $A$ : each entry $a_{i j}, i=1, \ldots, m$ and $j=1, \ldots, n$, is an independent, symmetric and bounded random variable with unknown distribution that takes values in $\left[a_{i j}-\hat{a}_{i j}, a_{i j}+\hat{a}_{i j}\right]$, where $a_{i j}$ is the mean value and $\hat{a}_{i j}$ is the range;

for the cost vector $c$ : each entry $c_{j}, j=1, \ldots, n$, takes values in $\left[c_{j}-d_{j}, c_{j}\right]$, where $d_{j} \geq 0$ represents the deviation from the nominal cost coefficient $c_{j}$.

Note that it is possible that $\hat{a}_{i j}=0$ or $d_{j}=0$. Intervals for the objective functions parameters are not centred in the nominal value (as we have for the constraints coefficients) because they consider the worst case scenario, which is represented by decreasing objective function coefficients.

The price of robustness is introduced through an integer upper bound value $\Gamma_{i}$, for $i=1, \ldots, m$, that takes value in the integer set $\left\{0,1,2, \ldots,\left|J_{i}\right|\right\}$. Sets $J_{i}=\left\{j: \hat{a}_{i j}>0\right\}$ allow us to count the number of parameters $a_{i j}$ with a non null range of variation, for each constraint $i$. The upper bound values $\Gamma_{i}$ enable us to control the trade-off between robustness and the level of conservatism of the solution, as we construct a solution which is robust to the change of up to $\Gamma_{i}$ parameters for each constraint $i$. If at most $\Gamma_{i}$ constraint parameters change, then we deterministically know that the robust solution will be feasible. Hence, $\Gamma_{i}$ is called the protection level of constraint $i$. Furthermore, Bertsimas and Sim (2003) show that even if more than $\Gamma_{i}$ parameters change, then the robust solution will be feasible with very high probability. We refer the interested reader to the paper for a detailed description of the probability bound.

Similarly another integer upper bound $\Gamma_{0}$ controls the trade-off between robustness and optimality: we search for a solution which optimises against all scenarios in which at most $\Gamma_{0}$ coefficients of the objective function vary in a way to maximally influence the objective value. This upper bound $\Gamma_{0}$ takes values in the integer set $\left\{0,1,2, \ldots,\left|J_{0}\right|\right\}$, where the set $J_{0}=\left\{j: d_{j}>0\right\}$ allows us to count the number of positive variations in the objective function parameters. In general a higher value of $\Gamma_{0}$ increases the level of robustness at the expenses of a worst objective function value. 
The robust counterpart of the problem defined by Equations (3.1a) to (3.1c) is:

$$
\begin{aligned}
& \max _{x} \quad c^{T} x-\max _{\left\{S_{0}: S_{0} \subseteq J_{0},\left|S_{0}\right| \leq \Gamma_{0}\right\}} \sum_{j \in S_{0}} d_{j}\left|x_{j}\right| \\
& \text { s.t. } \quad \sum_{j} a_{i j} x_{j}+\max _{\left\{S_{i}: S_{i} \subseteq J_{i},\left|S_{i}\right| \leq \Gamma_{i}\right\}} \sum_{j \in S_{i}} \hat{a}_{i j}\left|x_{j}\right| \leq b, \quad \forall i \\
& x_{j} \in \mathbb{Z} \quad j=1, \ldots, k .
\end{aligned}
$$

Bertsimas and Sim (2003) show that the robust counterpart defined by Equations (3.2a) to 3.2c) (which has $n$ variables and $m$ constraints) can be reformulated as a mixed-integer optimisation problem with $2 n+m+l$ variables and constraints, where $l=\sum_{i=0}^{m}\left|J_{i}\right|$ is the number of uncertain parameters.

If only the parameters $c$ of the objective function of a fully binary optimisation problem (i.e. $x_{j} \in\{0,1\}$, for all $j=1, \ldots, n$ ) are subject to uncertainty, Bertsimas and Sim (2003) solve the robust counterpart by solving at most $n+1$ instances of the nominal version of the problem. Therefore, the robust counterpart of a polynomially solvable binary optimisation problem remains polynomially solvable. This is valid for instance for the shortest path, minimum spanning tree, matching, etc., problems.

We now apply this robust approach with interval parameters and price of robustness to take into account for uncertainty in some (pseudo)-polynomial network pricing problems.

\subsection{The case of a single toll arc and multiple commodities}

Consider the pricing problem over a network in which the authority owns only one toll arc and multiple commodities are travelling and choosing whether using it or not. As there is only one toll arc there is no difference between the arc and path versions of the pricing problem. In Section 1.4.2 we saw that this problem is polynomial and that there is a straightforward solution procedure for it. This problem has also a formulation with binary variables such that we could use the result from Bertsimas and Sim (2003) to polynomially solve the robust counterpart for uncertainty in the objective function. Instead we will exploit some properties of the problem to develop better ad hoc solution methods.

Recall that the optimal toll $T \in\left\{M^{k}: k \in \mathcal{K}\right\} \cup\{0\}$, where $M^{k}=\max \left\{0, c_{o d}^{k}-c^{k}\right\}$, $\forall k \in \mathcal{K}$. Furthermore, once the toll $T$ is fixed, the choice of each commodity to use the toll arc or not can be easily obtained: $x^{k}=1$ if $M^{k} \geq T, 0$ otherwise, for each $k \in \mathcal{K}$. Let us define, for each $T \in \mathbb{R}$, the set of commodities using the toll arc if the toll is set to $T$ :

$$
\mathcal{K}(T)=\left\{k \in \mathcal{K}: M^{k} \geq T\right\} .
$$


The leader's revenue for a given toll value $T$ can be calculated as follows:

$$
\mathcal{R}(T)=T \sum_{k \in \mathcal{K}(T)} \eta^{k}
$$

We now consider different robust versions of this problem. Let us first make a consideration: we are solving worst case scenarios, which are represented by a decrease in the leader's revenue. Therefore we consider that either the demand or $M$ values of commodities might decrease, as both cases would result in a decrease of the revenue (see Equation (3.4)). Note that a decrease for $M$ values might be caused by a decrease of toll free path costs $c_{o d}^{k}$ and/or an increase of the toll arc fixed costs $c^{k}$.

Remark that with our approach we will be able to distinguish between uncertainty for demand and $M$ parameters of the problem, whilst if we used the approach proposed by Bertsimas and Sim (2003) for binary problems we would be obliged to consider uncertainty for the total objective function parameters, equal to the product of the cumulative demand and $M^{k}$ values (see Section 1.4.2).

\subsubsection{Worst case maximum revenue with interval demand}

Assume that the exact demand $\eta^{k}$ for each commodity $k$ is unknown, but an interval $I^{k}$ is known for it, such that $\eta^{k} \in I^{k}$. In particular the intervals have the following form:

$$
I^{k}=\left[\bar{\eta}^{k}-\Delta^{k}, \bar{\eta}^{k}\right] \quad \forall k \in \mathcal{K},
$$

where $\bar{\eta}^{k}$ is the nominal value and $\Delta^{k} \geq 0$ is the maximal possible reduction from the nominal value. We want to maximise the leader's revenue $\mathcal{R}$ in the worst case scenario, where the leader maximises the revenue over the toll value $T$, considering the minimum demand for commodities in the intervals $I^{k}$. Commodities' choice is defined by Equation (3.3). Finally, we consider that at most $\Gamma$ demand values can take a value lower than its nominal one, i.e. $\left|\left\{k: \eta^{k}<\bar{\eta}^{k}\right\}\right| \leq \Gamma$, with $\Gamma \in\{0,1, \ldots,|\mathcal{K}|\}$. The robust counterpart of the problem can be defined as follows:

$$
\max _{T} \min _{\left\{\eta^{k} \in I^{k}:\left\{k: \eta^{k}<\bar{\eta}^{k}\right\} \mid \leq \Gamma\right\}} \mathcal{R}(T, \eta) .
$$

Note that if $\Gamma=0$ we have the nominal version of the problem. The problem defined by (3.6) is polynomial, and we can prove the following proposition.

Proposition 3.3.1. The optimal toll value for the worst case maximum revenue NPP with a single toll arc and interval demand values, defined in Equation (3.6), belongs to $\left\{M^{k}: k \in\right.$ $\mathcal{K}\} \cup\{0\}$ and the optimal solution can be found in polynomial time.

Proof. First of all, consider a fixed toll value $T$. We notice that the set $\mathcal{K}(T)$ of commodities using the toll arc is independent from the demand level, as described above by Equation (3.3). The worst case leader's revenue is therefore obtained by choosing the $\Gamma$ 
biggest reductions of the demand for commodities using the toll arc for this toll value. For these commodities we set the demand equal to the corresponding interval lower bound. Let us define this set of commodities as $\Gamma(T)=\left\{k \in \mathcal{K}(T): \eta^{k}=\bar{\eta}^{k}-\Delta^{k}\right\}$. Second, one can easily see that, as for the nominal version of the problem, the optimal toll takes values in $\left\{M^{k}: k \in \mathcal{K}\right\} \cup\{0\}$. Then, the optimal solution can be found with the following polynomial algorithm.

Algorithm 3.3.1 (Robust algorithm for the single toll arc case and interval demand).

Step 1: order commodities $\Delta^{k}$ values, for $k \in \mathcal{K}$, in non increasing order, such that $a_{1} \geq a_{2} \geq \ldots \geq a_{|\mathcal{K}|}$ and $r_{1}(k)=l$ if $\Delta^{k}=a_{l}$.

Step 2: order commodities $M^{k}$ values, for $k \in \mathcal{K}$, in non decreasing order, such that $b_{1} \leq b_{2} \leq \ldots \leq b_{|\mathcal{K}|}$ and $r_{2}(k)=l$ if $M^{k}=b_{l}$.

\section{Step 3:}

initialisation $\left(T=b_{1}\right)$ :

1. $\mathcal{K}\left(b_{1}\right)=\mathcal{K}$;

2. $\Gamma\left(b_{1}\right)=\left\{k: r_{1}(k) \leq \Gamma\right\}$; set $\eta^{k}=\bar{\eta}^{k}-\Delta^{k}$ for $k \in \Gamma\left(b_{1}\right)$, and $\eta^{k}=\bar{\eta}^{k}$ for $k \notin \Gamma\left(b_{1}\right)$;

3. calculate the leader's revenue as $\mathcal{R}\left(b_{1}\right)=b_{1} \sum_{k \in \mathcal{K}} \eta^{k}$;

4. set $T^{*}=b_{1}$ and $\mathcal{R}^{*}=\mathcal{R}\left(b_{1}\right)$;

iteration $i\left(T=b_{i}\right)$, for $i=2, \ldots,|\mathcal{K}|$ :

1. $\mathcal{K}\left(b_{i}\right)=\mathcal{K}\left(b_{i-1}\right) \backslash\left\{k: r_{2}(k)=i-1\right\} ;$

2. if $\left\{k: r_{2}(k)=i-1\right\} \subseteq \Gamma\left(b_{i-1}\right)$ :

$$
\begin{gathered}
\Gamma\left(b_{i}\right)=\left(\Gamma\left(b_{i-1}\right) \backslash\{k\}\right) \cup\{h\} \\
\mathcal{R}\left(b_{i}\right)=b_{i}\left[\frac{\mathcal{R}\left(b_{i-1}\right)}{b_{i-1}}-\left(\bar{\eta}^{k}-\Delta^{k}\right)-\Delta^{h}\right]
\end{gathered}
$$

where $k: r_{2}(k)=i-1$ and $h=\arg \max \left\{\Delta^{p}: p \in \mathcal{K}\left(b_{i}\right)\right.$ and $\Delta^{p} \leq$ $\Delta^{q}$ where $\left.\Delta^{q}=\min \left\{\Delta^{s}: s \in \Gamma\left(b_{i-1}\right)\right\}\right\}$;

else:

$$
\begin{gathered}
\Gamma\left(b_{i}\right)=\Gamma\left(b_{i-1}\right) \\
\mathcal{R}\left(b_{i}\right)=b_{i}\left[\frac{\mathcal{R}\left(b_{i-1}\right)}{b_{i-1}}-\bar{\eta}^{k}\right],
\end{gathered}
$$

where $k: r_{2}(k)=i-1$;

3. if $b_{i}=b_{i-1}$ then $\mathcal{R}\left(b_{i}\right)=\min \left\{\mathcal{R}\left(b_{i}\right), \mathcal{R}\left(b_{i-1}\right)\right\}$;

4. $\mathcal{R}^{*}=\max \left\{\mathcal{R}^{*}, \mathcal{R}\left(b_{i}\right)\right\}$ and $T^{*}=\arg \max \left\{\mathcal{R}^{*}, \mathcal{R}\left(b_{i}\right)\right\}$. 
Note that a simple preprocessing is needed: all commodities with $M^{k} \leq 0$ should be removed from the set $\mathcal{K}$, if not the initialisation at Step 3 is not correct.

The first two steps of the algorithm can be executed in $\mathcal{O}(|\mathcal{K}| \log |\mathcal{K}|)$ and the third step in $\mathcal{O}(\Gamma|\mathcal{K}|)$, such that the whole algorithm runs in $\mathcal{O}(\max \{|\mathcal{K}| \log |\mathcal{K}|, \Gamma|\mathcal{K}|\})$.

We can easily notice that, if $\Gamma=|\mathcal{K}|$, the solution can be found solving the nominal version of the problem where $\eta^{k}=\bar{\eta}^{k}-\Delta^{k}$, for each $k \in \mathcal{K}$.

\subsubsection{Worst case maximum revenue with interval $M$ parameters}

Assume now that the demand is fixed and known, but values of the $M^{k}$ parameters lie in an interval $J^{k}$, similarly to the previous case:

$$
M^{k} \in J^{k}=\left[\bar{M}^{k}-\Omega^{k}, \bar{M}^{k}\right] \quad \forall k \in \mathcal{K},
$$

where $\bar{M}^{k}$ is the nominal value and $\Omega^{k} \geq 0$ is the maximal possible reduction from the nominal value. Note that as $M^{k}=\max \left\{0, c_{o d}^{k}-c^{k}\right\}$, the uncertainty on these values can be caused by the toll free path costs $c_{o d}^{k}$ and/or by the toll arc fixed $\operatorname{costs} c^{k}$.

Again similarly to the previous case, we want to maximise the leader's revenue $\mathcal{R}$ in the worst case scenario, where the leader maximises its revenue over the toll value $T$, considering the minimum $M^{k}$ value for commodities in the intervals $J^{k}$. Commodities' choices depend on the toll value and on their $M^{k}$ value, as described by Equation (3.3). We also consider that at most $\Gamma$ values of $M^{k}$ parameters can take a value lower than its nominal one, i.e. $\left|\left\{k: M^{k}<\bar{M}^{k}\right\}\right| \leq \Gamma$, with $\Gamma \in\{0,1, \ldots,|\mathcal{K}|\}(\Gamma=0$ represents the nominal version of the problem). The robust counterpart of the problem can be defined as follows:

$$
\max _{T} \min _{\left\{M^{k} \in J^{k}:\left|\left\{k: M^{k}<\bar{M}^{k}\right\}\right| \leq \Gamma\right\}} \mathcal{R}(T, M) .
$$

The problem defined by (3.8) is polynomial, and we can prove the following proposition.

Proposition 3.3.2. The optimal toll value for the worst case maximum revenue NPP with a single toll arc and interval $M$ parameters, defined by Equation 3.8 , belongs to $\left\{\bar{M}^{k}: k \in\right.$ $\mathcal{K}\} \cup\left\{\left(\bar{M}^{k}-\Omega^{k}\right): k \in \mathcal{K}\right\} \cup\{0\}$ and the optimal solution can be found in polynomial time.

Proof. First of all, consider a fixed toll value $T$. We know that a commodity $k$ is using the toll arc if $M^{k} \geq T$, but in this case the exact value of $M^{k}$ is unknown. Let us define the set of commodities that may use or not the toll arc depending on the value that $M^{k}$ takes in its corresponding interval:

$$
\Phi(T)=\left\{k \in \mathcal{K}: \bar{M}^{k} \geq T \text { and }\left(\bar{M}^{k}-\Omega^{k}\right)<T\right\}, \quad \forall T \in \mathbb{R} .
$$

This set represents commodities that will not use the toll arc if their $M^{k}$ value is equal to the lower bound of the corresponding $J^{k}$ interval. For the other commodities, i.e. 
commodities in $\mathcal{K} \backslash \Phi(T)$, the choice is fixed independently of the value that $M^{k}$ is taking in $J^{k}$. Therefore, the worst case leader's revenue is obtained by choosing the $\Gamma$ commodities in $\Phi(T)$ with the highest demand values and setting their $M^{k}$ value equal to the lower bound of the corresponding interval $J^{k}$. Let us define this set of commodities as $\Gamma(T)=\left\{k \in \Phi(T): M^{k}=\bar{M}^{k}-\Omega^{k}\right\}$. We have that $|\Gamma(T)| \leq \Gamma$.

Hence, optimal toll values are in the set of nominal values $\left\{\bar{M}^{k}: k \in \mathcal{K}\right\} \cup\{0\}$, as for the nominal version, and also in the set of interval lower bounds $\left\{\left(\bar{M}^{k}-\Omega^{k}\right): k \in \mathcal{K}\right\}$. Let us denote the set of toll candidates as $\left\{B^{h}: \exists k\right.$ s.t. $B^{h}=\bar{M}^{k}$ or $B^{h}=\left(\bar{M}^{k}-\Omega^{k}\right), k \in$ $\mathcal{K}\}$. The optimal solution can be found with the following polynomial algorithm.

Algorithm 3.3.2 (Robust algorithm for the single toll arc case and interval $M^{k}$ values).

Step 1: order commodities demand values, for $k \in \mathcal{K}$, in non increasing order, such that $a_{1} \geq a_{2} \geq \ldots \geq a_{|\mathcal{K}|}$ and $r_{1}(k)=l$ if $\eta^{k}=a_{l}$.

Step 2: order commodities $B^{h}$ values in non decreasing order, such that $b_{1} \leq b_{2} \leq \ldots \leq$ $b_{2|\mathcal{K}|}$ and $r_{2}(h)=l$ if $B^{h}=b_{l}$.

\section{Step 3:}

initialisation $\left(T=b_{1}\right)$ :

1. $\Phi\left(b_{1}\right)=\emptyset$;

2. $\Gamma\left(b_{1}\right)=\emptyset$;

3. $\mathcal{K}\left(b_{1}\right)=\mathcal{K}$;

4. calculate the leader's revenue as $\mathcal{R}\left(b_{1}\right)=b_{1} \sum_{k \in \mathcal{K}} \eta^{k}$;

5. set $T^{*}=b_{1}$ and $\mathcal{R}^{*}=\mathcal{R}\left(b_{1}\right)$;

iteration $i\left(T=b_{i}\right)$, for $i=2, \ldots, 2|\mathcal{K}|$ :

1. to calculate $\Phi\left(b_{i}\right)$ we need to distinguish if $b_{i-1}$ represents an interval lower bound (LB) or upper bound (UB) of its corresponding $J^{k}$ interval:

(a) if $b_{i-1}$ is a UB then:

$$
\Phi\left(b_{i}\right)=\Phi\left(b_{i-1}\right) \backslash\left\{k: \bar{M}^{k}=b_{i-1}\right\},
$$

(b) if $b_{i-1}$ is a LB then:

$$
\Phi\left(b_{i}\right)=\Phi\left(b_{i-1}\right) \cup\left\{h: \bar{M}^{h}-\Omega^{h}=b_{i-1}\right\},
$$

2. and calculate $\Gamma\left(b_{i}\right), \mathcal{K}\left(b_{i}\right)$ and $\mathcal{R}\left(b_{i}\right)$ accordingly:

(a) if $\{k\}=\Phi\left(b_{i-1}\right) \backslash \Phi\left(b_{i}\right)$ :

- if $k \in \Gamma\left(b_{i-1}\right)$ then:

$$
\Gamma\left(b_{i}\right)=\left(\Gamma\left(b_{i-1}\right) \backslash\{k\}\right) \cup\{t\},
$$




$$
\begin{gathered}
\mathcal{K}\left(b_{i}\right)=\mathcal{K}\left(b_{i-1}\right) \backslash\{t\}, \\
\mathcal{R}\left(b_{i}\right)=b_{i}\left(\frac{\mathcal{R}\left(b_{i-1}\right)}{b_{i-1}}-\eta^{t}\right)
\end{gathered}
$$

where $t=\arg \max \left\{\eta^{p}: p \in \Phi\left(b_{i}\right)\right.$ and $\eta^{p} \leq \eta^{q}$, where $\eta^{q}=$ $\left.\min \left\{\eta^{s}: s \in \Gamma\left(b_{i-1}\right)\right\}\right\}$;

- else:

$$
\begin{gathered}
\mathcal{K}\left(b_{i}\right)=\mathcal{K}\left(b_{i-1}\right) \backslash\{k\}, \\
\mathcal{R}\left(b_{i}\right)=b_{i}\left(\frac{\mathcal{R}\left(b_{i-1}\right)}{b_{i-1}}-\eta^{k}\right) ;
\end{gathered}
$$

(b) if $\{h\}=\Phi\left(b_{i}\right) \backslash \Phi\left(b_{i-1}\right)$ :

- if $\left|\Gamma\left(b_{i-1}\right)\right|<\Gamma$ then:

$$
\begin{gathered}
\Gamma\left(b_{i}\right)=\Gamma\left(b_{i-1}\right) \cup\{h\}, \\
\mathcal{K}\left(b_{i}\right)=\mathcal{K}\left(b_{i-1}\right) \backslash\{h\}, \\
\mathcal{R}\left(b_{i}\right)=b_{i}\left(\frac{\mathcal{R}\left(b_{i-1}\right)}{b_{i-1}}-\eta^{h}\right) ;
\end{gathered}
$$

- else:

- if $\eta^{h} \geq \eta^{l}$, where $l=\arg \min \left\{\eta^{p}: p \in \Gamma\left(b_{i-1}\right)\right\}$, then:

$$
\begin{gathered}
\Gamma\left(b_{i}\right)=\left(\Gamma\left(b_{i-1}\right) \cup\{h\}\right) \backslash\{l\}, \\
\mathcal{K}\left(b_{i}\right)=\left(\mathcal{K}\left(b_{i-1}\right) \backslash\{h\}\right) \cup\{l\}, \\
\mathcal{R}\left(b_{i}\right)=b_{i}\left(\frac{\mathcal{R}\left(b_{i-1}\right)}{b_{i-1}}-\eta^{h}+\eta^{l}\right) ;
\end{gathered}
$$

3. if $b_{i}=b_{i-1}$ then $\mathcal{R}\left(b_{i}\right)=\min \left\{\mathcal{R}\left(b_{i}\right), \mathcal{R}\left(b_{i-1}\right)\right\}$;

4. $\mathcal{R}^{*}=\max \left\{\mathcal{R}^{*}, \mathcal{R}\left(b_{i}\right)\right\}$ and $T^{*}=\arg \max \left\{\mathcal{R}^{*}, \mathcal{R}\left(b_{i}\right)\right\}$.

The first two steps of the algorithm can be executed in $\mathcal{O}(|\mathcal{K}| \log |\mathcal{K}|)$ and the third step in $\mathcal{O}(\Gamma|\mathcal{K}|)$, such that the whole algorithm runs in $\mathcal{O}(\max \{|\mathcal{K}| \log |\mathcal{K}|, \Gamma|\mathcal{K}|\})$.

Consider now how many toll values should be evaluated at Step 3 of the algorithm as candidates for the optimal solution, if we need to consider all of them or some can be excluded a priori via some preprocessing. We remark that in the worst case $2|\mathcal{K}|$ toll values must be evaluated in Step 3 of the solution algorithm. In fact we can find an example where the optimal toll $T^{*} \in\left\{\bar{M}^{k}: k \in \mathcal{K}\right\}$, and another example where $T^{*} \in\left\{\left(\bar{M}^{k}-\Omega^{k}\right): k \in \mathcal{K}\right\}$. Therefore we cannot exclude one of these sets a priori.

For both examples consider a network with one toll arc and two commodities, $k_{1}$ and $k_{2}$. We consider the following intervals for $M^{k}$ values: $M^{k_{1}} \in[5-2,5]$ and $M^{k_{2}} \in$ $[4-3,4]$, as represented in Figure 3.1. We allow only one $M^{k}$ value to be at its interval lower bound, i.e. $\Gamma=1$. 


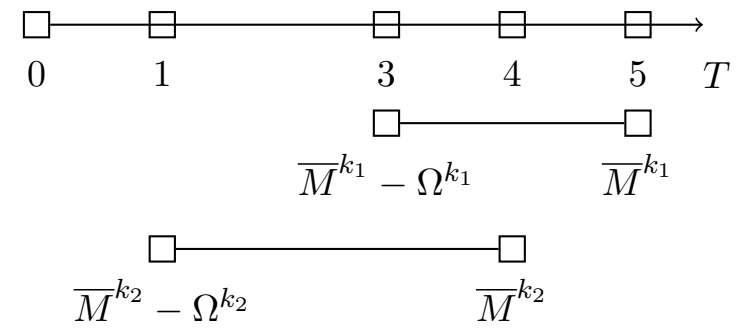

Figure 3.1: Examples: representation of $M^{k}$ intervals on the toll axis

Consider first an example in which the demand is equal to 7 and 8 for $k_{1}$ and $k_{2}$, respectively. If $T=4, \Phi(4)=\left\{k_{1}, k_{2}\right\}$, we then consider the commodity in $\Phi(4)$ with the biggest demand, which is $k_{2}$, and we take $M^{k_{2}}=1$. Therefore $\mathcal{K}(4)=\left\{k_{1}\right\}$, and we have a revenue of $\mathcal{R}(4)=4 \eta^{k_{1}}=28$. If $T=3, \Phi(3)=\left\{k_{2}\right\}$, and we take again $M^{k_{2}}=1$. We have $\mathcal{K}(3)=\left\{k_{1}\right\}$, with a revenue of $\mathcal{R}(3)=3 \eta^{k_{1}}=21$. We similarly obtain the worst case revenue for $T=1$, which is $\mathcal{R}(1)=15$, and for $T=5$, which is $\mathcal{R}(5)=0$. The optimal solution for this first example is in $T=4$, which is in $\left\{\bar{M}^{k}: k \in \mathcal{K}\right\}$.

Consider now a second example on the same network, where the demand is equal to 8 and 5 for $k_{1}$ and $k_{2}$ respectively. If $T=4$ we have again $\Phi(4)=\left\{k_{1}, k_{2}\right\}$, but now the commodity in $\Phi(4)$ with biggest demand is $k_{1}$, so we take $M^{k_{1}}=3$. The assignment set is $\mathcal{K}(4)=\left\{k_{2}\right\}$, for a revenue of $\mathcal{R}(4)=4 \eta^{k_{2}}=20$. If $T=3$ then $\Phi(3)=\left\{k_{2}\right\}$ such that we take $M^{k_{2}}=1$. We have $\mathcal{K}(3)=\left\{k_{1}\right\}$, with a revenue of $\mathcal{R}(3)=3 \eta^{k_{1}}=24$. For $T=1$ we obtain $\mathcal{R}(1)=13$, and for $T=5$ we obtain $\mathcal{R}(5)=0$. The optimal solution for this second example is in $T=3$, which is in $\left\{\left(\bar{M}^{k}-\Omega^{k}\right): k \in \mathcal{K}\right\}$.

Proposition 3.3.3 (Toll dominance). Consider two "consecutive" toll candidates $T_{1}$ and $T_{2}$, i.e. $T_{1}<T_{2}, T_{1}, T_{2} \in\left\{\bar{M}^{k}: k \in \mathcal{K}\right\} \cup\left\{\left(\bar{M}^{k}-\Omega^{k}\right): k \in \mathcal{K}\right\}$, and $] T_{1}, T_{2}\left[\cap\left(\left\{\bar{M}^{k}: k \in\right.\right.\right.$ $\left.\mathcal{K}\} \cup\left\{\left(\bar{M}^{k}-\Omega^{k}\right): k \in \mathcal{K}\right\}\right)=\emptyset$. If $\Phi\left(T_{2}\right) \subseteq \Phi\left(T_{1}\right)$, then $T_{2}$ dominates $T_{1}$ for $\Gamma \geq\left|\Phi\left(T_{1}\right)\right|$, in the sense that the leader's revenue obtained for $T_{2}$ with the solution algorithm 3.3 .2 is not smaller than the same value for $T_{1}$.

Proof. Let us denote $\mathcal{R}\left(T_{1}\right)$ and $\mathcal{R}\left(T_{2}\right)$ as the leader's revenue obtained with the solution algorithm 3.3.2, for $T_{1}$ and $T_{2}$ respectively. From Equation (3.4), we have:

$$
\mathcal{R}\left(T_{1}\right)=T_{1} \sum_{k \in \mathcal{K}\left(T_{1}\right)} \eta^{k} \quad \text { and } \quad \mathcal{R}\left(T_{2}\right)=T_{2} \sum_{k \in \mathcal{K}\left(T_{2}\right)} \eta^{k}
$$

We want to prove that $\mathcal{R}\left(T_{1}\right) \leq \mathcal{R}\left(T_{2}\right)$. As $T_{1}<T_{2}$, if we prove that $\mathcal{K}\left(T_{1}\right) \subseteq \mathcal{K}\left(T_{2}\right)$, the proposition is verified.

As $\Phi\left(T_{2}\right) \subseteq \Phi\left(T_{1}\right)$, we have $\left|\Phi\left(T_{2}\right)\right| \leq\left|\Phi\left(T_{1}\right)\right|$. Therefore $\Gamma \geq\left|\Phi\left(T_{1}\right)\right| \geq\left|\Phi\left(T_{2}\right)\right|$ and we can easily see that, for each $k \in \mathcal{K}$, we have:

$$
\text { if } k \in \Phi\left(T_{1}\right) \text { then } k \notin \mathcal{K}\left(T_{1}\right) \text {, }
$$


and similarly:

$$
\text { if } k \in \Phi\left(T_{2}\right) \text { then } k \notin \mathcal{K}\left(T_{2}\right),
$$

where sets $\mathcal{K}(T)$, for $T_{1}$ and $T_{2}$, are determined at Step 3 of the solution algorithm. This means that $\Phi\left(T_{1}\right) \cap \mathcal{K}\left(T_{1}\right)=\emptyset$ and $\Phi\left(T_{2}\right) \cap \mathcal{K}\left(T_{2}\right)=\emptyset$.

Define now, for a fixed toll value, the set of commodities choosing the toll free path, after (at most) $\Gamma$ values of $M^{k}$ are set to their interval lower bound as from Step 3 of the solution algorithm:

$$
\mathcal{K}_{o d}(T)=\mathcal{K} \backslash \mathcal{K}(T)=\left\{k \in \mathcal{K}: \bar{M}^{k}<T\right\} \cup\{k \in \mathcal{K}: k \in \Phi(T) \text { and } k \notin \mathcal{K}(T)\} .
$$

For $T_{1}$ we saw that $\Phi\left(T_{1}\right) \cap \mathcal{K}\left(T_{1}\right)=\emptyset$, such that $\mathcal{K}_{\text {od }}\left(T_{1}\right)=\left\{k \in \mathcal{K}: \bar{M}^{k}<T_{1}\right\} \cup \Phi\left(T_{1}\right)$. Similarly $\mathcal{K}_{\text {od }}\left(T_{2}\right)=\left\{k \in \mathcal{K}: \bar{M}^{k}<T_{2}\right\} \cup \Phi\left(T_{2}\right)$.

From the construction of these sets we have that, for $T \in\left\{T_{1}, T_{2}\right\}, \mathcal{K}=\mathcal{K}_{\text {od }}(T) \cup$ $\mathcal{K}(T)$ and $\mathcal{K}_{\text {od }}(T) \cap \mathcal{K}(T)=\emptyset$, such that sets $\mathcal{K}_{\text {od }}(T)$ and $\mathcal{K}(T)$ represent a partition of $\mathcal{K}$. We therefore have two partitions as represented in Figure 3.2.
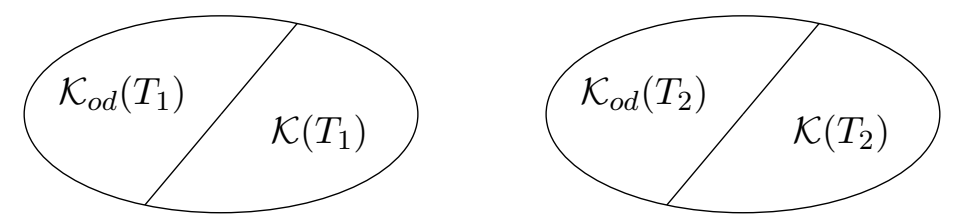

Figure 3.2: Partitions of the commodities set $\mathcal{K}$ for $T_{1}$ and $T_{2}$

From the hypothesis we know that $\nexists k: \bar{M}^{k}<T_{2}$ and $\bar{M}^{k}-\Omega^{k}>T_{1}$, which means $\nexists k: k \in \mathcal{K}_{\text {od }}\left(T_{2}\right)$ and $k \in \mathcal{K}\left(T_{1}\right)$. We therefore have $\mathcal{K}_{\text {od }}\left(T_{2}\right) \cap \mathcal{K}\left(T_{1}\right)=\emptyset$.

Consider now a commodity $k \in \mathcal{K}\left(T_{1}\right)$ : this commodity is either in $\mathcal{K}_{o d}\left(T_{2}\right)$ or in $\mathcal{K}\left(T_{2}\right)$, but sets $\mathcal{K}\left(T_{1}\right)$ and $\mathcal{K}_{\text {od }}\left(T_{2}\right)$ are disjoint, so we can conclude that $k \in \mathcal{K}\left(T_{2}\right)$. This proves that $\mathcal{K}\left(T_{1}\right) \subseteq \mathcal{K}\left(T_{2}\right)$.

Therefore, if we have two values $T_{1}$ and $T_{2}$ satisfying Proposition 3.3 .3 hypothesis, we can eliminate $T_{1}$ from the list of candidates of Step 3 of the solution algorithm without deteriorating the optimal solution value.

Finally we can remark that in some cases the biggest toll candidate value $N=$ $\max _{k} \bar{M}^{k}$ gives a null leader's revenue. This is the case if $\Gamma \geq \kappa$, where $\kappa$ is the number of commodities $k$ for which $\bar{M}^{k}=N$. In fact, with the assumption that $\Omega^{k}>0$ for all $k$ such that $\bar{M}^{k}=N$, we have that $|\Phi(N)|=\kappa$ and $\mathcal{K}(N)=\emptyset$.

\subsection{The case of a single commodity and multiple toll arcs}

Consider the path pricing problem with only one commodity that we described in Section 1.5.3. We saw that it is polynomial, contrary to its arc version. As done in the 
previous section, we exploit the properties of the problem to develop ad hoc solution methods for its robust counterparts.

In this problem we have the authority imposing a toll $T_{a}$ on toll paths $a \in \mathcal{A}$ of the network, and one commodity deciding if taking one of these paths (with a fixed cost of $c_{a}$ ) or the toll free path with a cost of $c_{o d}$. For each toll path we fix $M_{a}=\max \left\{0, c_{o d}-c_{a}\right\}$. In Section 1.5.3 we showed that the best toll choices for the authority are, for each toll path $a \in \mathcal{A}$ :

$$
T_{a}= \begin{cases}M_{a} & \text { if } a=\arg \max _{a} M_{a} \\ \infty & \text { otherwise }\end{cases}
$$

implying the following commodity's choices:

$$
x_{a}= \begin{cases}1 & \text { if } a=\arg \max _{a} M_{a} \\ 0 & \text { otherwise }\end{cases}
$$

The optimal solution value is $\mathcal{R}=\eta \max _{a} M_{a}$, where $\eta$ is the commodity's demand.

We now consider different robust versions of the problem, again considering the cases in which the optimal solution value may deteriorate: a decrease in the commodity's demand and a decrease in the $M_{a}$ values.

\subsubsection{Worst case maximum revenue with interval demand}

Assume that the demand for the commodity is unknown but restricted to belong to an interval:

$$
\eta \in I=[\bar{\eta}-\Delta, \bar{\eta}],
$$

where $\bar{\eta}$ is the nominal value and $\Delta \geq 0$ is the maximal possible reduction from the nominal value. As for the problems described in the previous section, we want to maximise the leader's revenue in the worst case scenario, which is given by solving the following problem:

$$
\max _{T} \min _{\eta \in I} \mathcal{R}(T, \eta)
$$

Note that in this problem it does not really make sense to consider a $\Gamma$ upper bound for the number of uncertain values that are allowed to be lower than their nominal value. In fact there is only one uncertain parameter, and either we allow it to take any value in the interval (and we have the robust counterpart) or not (and we have the nominal version).

Proposition 3.4.1. The optimal solution of the worst case maximum revenue single commodity path NPP with interval demand defined by Equation (3.17) is the same as for the nominal version of the problem, and the optimal solution value is $(\bar{\eta}-\Delta) \max _{a} M_{a}$. 
Proof. Since the optimal solution of the nominal problem does not depend on the demand level (see Equations (3.14) and (3.15)), the optimal solution of the robust problem can be obtained solving the nominal version with $\eta=\bar{\eta}-\Delta$ (which corresponds to the worst case scenario for the demand), obtaining an optimal solution value of $(\bar{\eta}-\Delta) \max _{a} M_{a}$.

\subsubsection{Worst case maximum revenue with interval $M$ parameters}

Assume now that the demand is fixed and known, but values of $M_{a}$ parameters lie in an interval $J_{a}$ :

$$
M_{a} \in J_{a}=\left[\bar{M}_{a}-\Omega_{a}, \bar{M}_{a}\right] \quad \forall a \in \mathcal{A},
$$

where $\bar{M}_{a}$ is the nominal value and $\Omega_{a} \geq 0$ is the maximal possible reduction from the nominal value. Note that $M_{a}=\max \left\{0, c_{o d}-c_{a}\right\}$, such that the uncertainty on these values can be caused by the toll free path $\cos t c_{o d}$ and/or the fixed cost of toll paths $c_{a}$. As there is only one toll free path cost we need to distinguish two cases: if the only uncertain value is the toll free path cost, the problem is simpler than if there is uncertainty on toll paths fixed cost values.

Consider first that all the fixed cost of toll paths $c_{a}$ are fixed and known, but the toll free path cost belongs to an interval $\left[\bar{c}_{o d}-\Omega, \bar{c}_{o d}\right]$. In this case we have that the maximal possible reduction of $M_{a}$ parameters from their nominal value is $\Omega_{a}=\Omega$, for all $a \in \mathcal{A}$. The worst case scenario of this problem is defined by the following problem:

$$
\max _{T} \min _{\left\{M_{a} \in J_{a}=\left[\bar{M}_{a}-\Omega, \bar{M}_{a}\right]\right\}} \mathcal{R}(T, M) .
$$

As for the interval demand problem described in the previous section, also for this problem it does not make sense to consider a $\Gamma$ upper bound value.

Proposition 3.4.2. The optimal solution of the worst case maximum revenue single commodity path NPP with interval toll free path cost defined by Equation (3.19) is the same as for the nominal version of the problem, and the optimal solution value is $\eta\left(\max _{a} \bar{M}_{a}-\Omega\right)$.

Proof. In this case we obtain the worst case scenario by considering that all $M_{a}$ values are equal to their interval lower bound, having $M_{a}=\bar{M}_{a}-\Omega$, for all $a \in \mathcal{A}$. Therefore the optimal solution can be obtained solving the deterministic version of the problem with $M_{a}=\bar{M}_{a}-\Omega$, obtaining an optimal solution value of $\eta \max _{a}\left(\bar{M}_{a}-\Omega\right)=$ $\eta\left(\max _{a} \bar{M}_{a}-\Omega\right)$, as the value $\Omega$ does not depend on the toll path $a$. Notice that the optimal solution is the same as the one of the nominal version of the problem, as $\arg \max _{a}\left(\bar{M}_{a}-\Omega\right)=\arg \max _{a} \bar{M}_{a}$.

Consider now that (also) the fixed costs of toll paths $c_{a}$ are unknown, such that intervals $J_{a}$ have a different width for each $a \in \mathcal{A}$, as described by Equation (3.18). In this case we consider that at most $\Gamma$ values of $M_{a}$ parameters can take a value lower 
than its nominal one, i.e. $\left|\left\{a: M_{a}<\bar{M}_{a}\right\}\right| \leq \Gamma$, with $\Gamma \in\{0,1, \ldots,|\mathcal{A}|\}$. If we want to maximise the leader's revenue in the worst case scenario we obtain the following robust problem:

$$
\max _{T} \min _{\left\{M_{a} \in J_{a}:\left|\left\{k: M_{a}<\bar{M}_{a}\right\}\right| \leq \Gamma\right\}} \mathcal{R}(T, M) .
$$

Proposition 3.4.3. The worst case maximum revenue path NPP with a single commodity and interval $M$ parameters defined by Equation (3.20) can be solved in polynomial time.

Proof. The optimal solution can be found with the following polynomial algorithm.

Algorithm 3.4.1 (Robust algorithm for the single commodity case and interval $M_{a}$ ).

Step 1: order toll paths $a \in \mathcal{A}$ corresponding to decreasing nominal $\bar{M}_{a}$ values, such that $\bar{M}_{a_{1}} \geq \bar{M}_{a_{2}} \geq \ldots \geq \bar{M}_{|\mathcal{A}|}$.

Step 2: for each $a \in \mathcal{A}$ set the $M_{a}$ values as following:

- $M_{a}=\max \left\{0, \bar{M}_{a}-\Omega_{a}\right\}$ for the $\Gamma$ first toll paths;

- $M_{a}=\bar{M}_{a}$ for the others.

Step 3: determine the optimal solution using Equations 3.14) and 3.15.

The first step of the algorithm can be executed in $\mathcal{O}(|\mathcal{A}| \log |\mathcal{A}|)$ and the second and the third step in $\mathcal{O}(|\mathcal{A}|)$, such that the algorithm runs in $\mathcal{O}(|\mathcal{A}| \log |\mathcal{A}|)$.

\subsection{The case of a unit toll}

In Chapter 2 we introduced two variants of the unit toll problem (with multiple commodities and multiple toll arcs): the constant version CT-NPP where all tolls are equal for all toll arcs, $T_{a}=T, \forall a \in \mathcal{A}_{1}$, and the proportional version PT-NPP where tolls are proportional to a given parameter for each arc, $T_{a}=\mu_{a} T, \mu_{a} \in \mathbb{N}, \forall a \in \mathcal{A}_{1}$. We showed that the CT-NPP is polynomial, whilst the PT-NPP is pseudo-polynomial.

These problems are very strongly connected with the single toll arc pricing problem and their deterministic versions can be solved with a similar algorithm. We now extend some results presented in Section 3.3 for the robust version of the single toll arc case to the robust version of the unit toll case. We will only consider the PT-NPP as the same results hold for the CT-NPP replacing $\mu_{a}$ with $\nu_{a}$ (see Section 2.2.2 for the parameters' definition).

Recall that the optimal toll value for PT-NPP belongs to the discrete set $\left\{B_{a}^{k}: a \in\right.$ $\mathcal{A}, k \in \mathcal{K}\} \cup\{0\}$, where $B_{a}^{k}$ are the breakpoints' values defined in Section 2.3.2, Equation (2.5). Similarly as for the single toll arc case, for a fixed toll value, we can determine the set of commodities using a toll arc as follows:

$$
\mathcal{K}(T)=\left\{k \in \mathcal{K}: \exists a \in \mathcal{A} \text { such that } B_{a}^{k} \geq T\right\}
$$


The leader's revenue for a given toll value $T$ can be calculated as follows:

$$
\mathcal{R}(T)=T \sum_{k \in \mathcal{K}(T)} \eta^{k} \mu_{a}
$$

where $a=\arg \min _{a \in \mathcal{A}: B_{a}^{k} \geq T} B_{a}^{k}$.

We now consider the robust version of this problem, when parameters uncertainty may result in a deterioration of the leader's revenue. As for the single toll arc case, the leader's revenue is influenced by the demand and the breakpoints values.

\subsubsection{Worst case maximum revenue with interval demand}

As for the other cases, assume that the demand $\eta^{k}$ for each commodity $k$ is unknown and constrained to lie in an interval $I^{k}$, as follows:

$$
\eta^{k} \in I^{k}=\left[\bar{\eta}^{k}-\Delta^{k}, \bar{\eta}^{k}\right] \quad \forall k \in \mathcal{K},
$$

where $\bar{\eta}^{k}$ is the nominal value and $\Delta^{k} \geq 0$ is the maximal possible reduction from the nominal value. We want to maximise the leader's revenue $\mathcal{R}$ in the worst case scenario, where the leader maximises the revenue over the toll value $T$, considering the minimum demand for commodities in the intervals $I^{k}$ and knowing that the set of commodities paying tolls can be determined with Equation (3.21). Finally, we consider that at most $\Gamma$ demand values can take a value lower than its nominal one, i.e. $\mid\{k$ : $\left.\eta^{k}<\bar{\eta}^{k}\right\} \mid \leq \Gamma$, with $\Gamma \in\{0,1, \ldots,|\mathcal{K}|\}$. The robust counterpart of the problem can be defined as follows:

$$
\max _{T} \min _{\left\{\eta^{k} \in I^{k}:\left|\left\{k: \eta^{k}<\bar{\eta}^{k}\right\}\right| \leq \Gamma\right\}} \mathcal{R}(T, \eta) .
$$

Note that if $\Gamma=0$ we have the nominal version of the problem. The problem defined by (3.24) is pseudo-polynomial, and we can prove the following proposition.

Proposition 3.5.1. The optimal toll value for the worst case maximum revenue PT-NPP with interval demand values, defined in Equation (3.24), belongs to $\left\{B_{a}^{k}: a \in \mathcal{A}, k \in \mathcal{K}\right\} \cup\{0\}$ and the optimal solution can be found in pseudo-polynomial time.

Proof. The proof is similar to the proof of Proposition 3.3.1 as the set of commodities $\mathcal{K}(T)$ is independent from the demand level. Also the solution algorithm is similar, replacing the set of toll candidates with the set $\left\{B_{a}^{k}: a \in \mathcal{A}, k \in \mathcal{K}\right\} \cup\{0\}$. Finding this set values is pseudo-polynomial (see Section 2.3.1) such that the whole algorithm runs in pseudo-polynomial time. Due to the complex notation and the fact that the algorithm is an extension of Algorithm 3.3.1. we do not go into more details.

Note that for the CT-NPP finding the set of candidates for the toll is polynomially solvable such that its robust counterpart with interval demand is polynomially solvable, as for the single toll arc case. 


\subsubsection{Worst case maximum revenue with interval $M$ parameters}

Assume now that the demand is fixed and known, but values of $M_{a}^{k}$ parameters lie in an interval $J_{a}^{k}$ :

$$
M_{a}^{k} \in J_{a}^{k}=\left[\bar{M}_{a}^{k}-\Omega_{a}^{k}, \bar{M}_{a}^{k}\right] \quad \forall a \in \mathcal{A}, \forall k \in \mathcal{K},
$$

where $\bar{M}_{a}^{k}$ is the nominal value and $\Omega_{a}^{k} \geq 0$ is the maximal possible reduction from the nominal value. As for the previous case, we want to maximise the leader's revenue $\mathcal{R}$ in the worst case scenario, consider that at most $\Gamma M$ values can take a value lower than its nominal one, i.e. $\left|\left\{(a, k): M_{a}^{k}<\bar{M}_{a}^{k}\right\}\right| \leq \Gamma$, with $\Gamma \in\{0,1, \ldots,|\mathcal{A}||\mathcal{K}|\}$. The robust counterpart of the problem can be defined as follows:

$$
\max _{T} \min _{\left\{M_{a}^{k} \in J_{a}^{k}:\left|\left\{(a, k): M_{a}^{k}<\bar{M}_{a}^{k}\right\}\right| \leq \Gamma\right\}} \mathcal{R}(T, M) .
$$

Note that if $\Gamma=0$ we have the nominal version of the problem.

For the nominal version of the problem we saw that toll candidates are represented by breakpoints' values, which are calculated considering two toll paths, as follows (for more details see Section 2.3.2):

$$
B_{a_{i}}^{k}=\frac{c_{a_{i+1}}^{k}-c_{a_{i}}^{k}}{\mu_{a_{i}}^{k}-\mu_{a_{i+1}}^{k}}=\frac{M_{a_{i}}^{k}-M_{a_{i+1}}^{k}}{\mu_{a_{i}}^{k}-\mu_{a_{i+1}}^{k}} \quad \forall k \in \mathcal{K}, i=1,2, \ldots,\left|\mathcal{A}^{k}\right| .
$$

If we consider that parameters $M_{a}^{k}$ are uncertain and constrained to lie in intervals, the intervals in which breakpoints belong might be affected by a combination of two intervals. Therefore in the robust approach we need to consider 4 possible values for each breakpoint, corresponding to the lower and upper bound of the intervals of the two $M$ values defining it. We can then use a similar algorithm as the one provided in Section 3.3.2 for the single toll arc case. Again, for PT-NPP the algorithm complexity will be dominated by finding all non-dominated toll paths to construct the breakpoints' set, which is pseudo-polynomial, but it is polynomial for CT-NPP. Given the complex notation and the fact that the solution algorithm is a modification of Algorithm 3.3.2, as for the interval demand case we do not go into further details. 


\section{Part III}

\section{An NP-hard pricing problem}





\section{Chapter 4}

\section{A Dantzig-Wolfe Reformulation for the NPP with Connected Toll Arcs}

\subsection{Introduction}

In this third part of the thesis we consider the Network Pricing Problem with connected toll arcs or Highway Problem introduced in Section 1.5.4. As this problem proved to be difficult, we tried to develop new stronger formulations for it, and efficient algorithms to solve them. In this chapter we present a Dantzig-Wolfe reformulation and an extended mixed-integer linear formulation, providing theoretical and numerical results to assess their strength and performance.

This chapter is organised as follows. At the beginning of the chapter we present the Dantzig-Wolfe reformulation. First of all we briefly introduce the general methodology. We then apply it to the NPP with connected toll arcs: we detail the master problem and the arising subproblem used to solve it. Even though the subproblem is non-linear, a simple solution procedure is proposed for it and integrated in a column generation algorithm. Secondly we exploit the structure of the problem revealed by the Dantzig-Wolfe reformulation to develop an extended compact linear formulation with a polynomial but very large number of variables and constraints. We then theoretically compare these new formulations with a mixed-integer linear formulation proposed in the literature. In the second half of the chapter further improvements for the column generation algorithm are discussed, as initialisation, stabilisation procedure and early stopping criteria for the pricing problem. Farkas pricing is introduced to solve possible infeasibility of the master problem. Numerical results are presented in each section, showing the performance of the different proposed strategies. In Section 4.10 we combine all the proposed schemes and tune the column generation algorithm to provide its best configuration. Furthermore, a class of valid inequalities from the literature has also been analysed and reformulated to be included in the algorithm.

This chapter focuses on the linear relaxation of the problem, whilst in Chapter 5 we introduce heuristics ideas to find good integer solutions. These elements are then 
combined introducing different branching strategies and presenting a full Branch-(andCut)-and-Price framework to solve the integer problem.

\subsection{Dantzig-Wolfe reformulation of an Integer Problem}

In this section we provide a brief explanation of the Dantzig-Wolfe reformulation from the literature. It is a methodology that was proposed by Dantzig and Wolfe (1960) that allows us to reformulate a problem in the variables space, such that a large number of variables arises and a column generation approach is therefore applied to solve it.

Consider an Integer Problem (IP) in the form:

$$
(\mathrm{IP})=\max \left\{\sum_{k=1}^{K} c^{k} x^{k}: \sum_{k=1}^{K} A^{k} x^{k}=b, x^{k} \in \mathcal{X}^{k} \text { for } k=1, \ldots, K\right\},
$$

where $\mathcal{X}^{k}=\left\{x^{k} \in \mathbb{Z}_{+}^{n_{k}}: D^{k} x^{k} \leq d^{k}\right\}$ for $k=1, \ldots, K$.

Each set $\mathcal{X}^{k}, k=1, \ldots, K$, contains a large but finite set of points $x^{k, j}$, where $j=$ $1, \ldots, J_{k}$, such that it can be represented as the set of convex combinations of its points with integer coefficients, as follows:

$$
\mathcal{X}^{k}=\left\{x^{k} \in \mathbb{Z}_{+}^{n_{k}}: x^{k}=\sum_{j=1}^{J_{k}} \lambda^{k, j} x^{k, j}, \sum_{j=1}^{J_{k}} \lambda^{k, j}=1, \lambda^{k, j} \in\{0,1\} \text { for } j=1, \ldots, J_{k}\right\} .
$$

Note that sets $\mathcal{X}^{k}$ are discrete (we are solving a full integer problem) and we therefore choose exactly one point in the convex combination (variables $\lambda$ are binary). We reformulate our integer problem using this convex combination of the points of sets $\mathcal{X}^{k}$. The (IP) can be written as follows:

$$
\begin{array}{ll}
\max & \sum_{k=1}^{K} \sum_{j=1}^{J_{k}} c^{k} x^{k, j} \lambda^{k, j}, \\
& \sum_{k=1}^{K} \sum_{j=1}^{J_{k}} A^{k} x^{k, j} \lambda^{k, j}=b, \\
& \sum_{j=1}^{J_{k}} \lambda^{k, j}=1 \\
\lambda^{k, j} \in\{0,1\} & \forall k \in\{1, \ldots, K\}, \\
& \forall j \in\left\{1, \ldots, J_{k}\right\}, \forall k \in\{1, \ldots, K\} .
\end{array}
$$

This problem is usually called Master Problem (MP), and decision variables are the $\lambda^{k, j}$. Constraints $4.3 \mathrm{c}$ ) are known as convexity constraints.

To solve the integer problem (MP) we first solve its linear relaxation, and then we can for instance perform an intelligent enumeration of possible integer solution within 
a Branch-and-Bound framework. The linear relaxation of (MP) is as follows:

(MP-LR)

$$
\begin{array}{ll}
\max & \sum_{k=1}^{K} \sum_{j=1}^{J_{k}} c^{k} x^{k, j} \lambda^{k, j}, \\
& \sum_{k=1}^{K} \sum_{j=1}^{J_{k}} A^{k} x^{k, j} \lambda^{k, j}=b, \quad \forall k \in\{1, \ldots, K\}, \\
& \sum_{j=1}^{J_{k}} \lambda^{k, j}=1 \\
\lambda^{k, j} \geq 0 & \forall j \in\left\{1, \ldots, J_{k}\right\}, \forall k \in\{1, \ldots, K\},
\end{array}
$$

where there is a column (i.e. variable) $\lambda^{k, j}$ for each point of $\mathcal{X}^{k}$, for all $k \in\{1, \ldots, K\}$. We associate dual variables as follows:

- variables $\pi_{i}$ for $i=1, \ldots, m$ (where $m$ is the number of rows of matrix $A$ ) for constraints $4.4 \mathrm{~b})$;

- variables $\mu_{k}$ for $k=1, \ldots, K$ for constraints $4.4 \mathrm{c}$.

To solve (MP-LR), instead of including all columns/variables and use the standard step of the simplex algorithm to price them and choose which one is entering the basis, we transform this step into a sequence of optimisation problems looking for the best column to add (column generation algorithm).

We start by selecting a subset of columns and we solve the so-called restricted linear relaxation of (MP), containing only these columns. We therefore define a subproblem to verify optimality with respect to the original problem (with the full set of columns) or to find more columns to include, and we iterate.

The subproblem consists in checking whether the optimal dual vector $\left(\pi^{*}, \mu^{*}\right)$ of the linear relaxation of the restricted (MP) is dual feasible for the linear relaxation of the unrestricted or complete (MP). To do so we verify if the reduced price of each column (i.e. each $k \in K$ and each $x \in \mathcal{X}^{k}$ ) is non positive. We can formulate this condition as an optimisation problem, which determines the column maximising the reduced price. This problem, called Subproblem (SP) or pricing problem (as it prices columns with respect to their reduced price) is:

$$
(\mathrm{SP})=\max \left\{\left(c^{k}-\pi^{*} A^{k}\right) x-\mu_{k}^{*}: x \in \mathcal{X}^{k}, k=1, \ldots, K\right\} .
$$

If the optimal solution of (SP) is zero or negative, we have the proof that the optimal solution of the linear relaxation of the current restricted (MP) is optimal also for the linear relaxation of the unrestricted (MP), as there are no columns with positive reduced price that might improve the solution. If (SP) has a positive optimal solution, there is at least one column corresponding to this optimal solution who has a positive reduced 
price. We introduce this column with its coefficients into the linear relaxation of restricted (MP) and we iterate. Note that we may also add more than one column (with positive reduced price) at each iteration. The (SP) includes constraints that have been eliminated from the (MP), such as constraints defining sets $\mathcal{X}^{k}$ in our case.

When using a Branch-and-Bound scheme to solve an integer problem this procedure is repeated to solve the linear problem associated to each node of the tree, leading to a methodology called Branch-and-Price.

Let us note $Z_{M P}^{*}$ as the optimal value of the linear relaxation of the unrestricted (MP) (Equations (4.4a to (4.4d) and $R P_{k}$ as the optimal value of the $(\mathrm{SP})_{k}$, for each $k=1, \ldots, K$, corresponding to the optimal solution of the linear relaxation of the current restricted (MP). From $(\mathrm{SP})_{k}$ we know that $R P_{k} \geq\left(c^{k}-\pi^{*} A^{k}\right) x-\mu_{k}^{*}$ for all $x \in \mathcal{X}^{k}$, and therefore $\left(c^{k}-\pi^{*} A^{k}\right) x-\mu_{k}^{*}-R P_{k} \leq 0$ for all $x \in \mathcal{X}^{k}$. If we denote $R P=\left(R P_{1}, \ldots, R P_{K}\right)$, we have that $\left(\pi^{*}, \mu^{*}+R P\right)$ is dual feasible for the linear relaxation of $(\mathrm{MP})$, such that:

$$
Z_{M P}^{*} \leq \pi^{*} b+\sum_{k=1}^{K} \mu_{k}^{*}+\sum_{k=1}^{K} R P_{k} .
$$

The right-hand side value of Equation (4.6) represents an upper bound on the optimal value of the linear relaxation of the unrestricted (MP) and can be calculated at each iteration of the column generation algorithm.

For more details we refer to the book by Wolsey (1998, chap. 11). A didactic introduction to the use of column generation technique in linear and integer programming together with examples has been provided by Desrosiers and Lübbecke (2005). For another tutorial on column generation methodology we refer to Feillet (2010), where the author provides a didactic explanation with an example of a Vehicle Routing Problem with Time Windows (VRPTW). More advanced issues that arise when using this technique, such as different branching strategies, initialisation alternatives or stabilisation procedures, have been discussed for instance in Barnhart et al. (1998), Vanderbeck (2000) and Lübbecke and Desrosiers (2005). We present some of them in details applied to the NPP in the second half of this chapter and in Chapter 5 (for branching strategies).

\subsection{A Dantzig-Wolfe reformulation for the NPP with connected toll arcs}

\subsubsection{Master Problem}

We consider the NPP with connected toll arcs and we propose a Dantzig-Wolfe reformulation (HPDW) starting from the single level non linear model (HPNL) presented in Section 1.5.6. The formulation is as follows: 
(HPNL)

$$
\begin{array}{llr}
\max _{T, x, p} & \sum_{a \in \mathcal{A}} \sum_{k \in \mathcal{K}} \eta^{k} p_{a}^{k}, \\
\text { s.t. } & \sum_{a \in \mathcal{A}}\left[\left(c_{a}^{k}-c_{o d}^{k}\right) x_{a}^{k}+p_{a}^{k}\right]-T_{b} \leq c_{b}^{k}-c_{o d}^{k} & \forall k \in \mathcal{K}, \forall b \in \mathcal{A}, \\
& \sum_{a \in \mathcal{A}}\left[\left(c_{a}^{k}-c_{o d}^{k}\right) x_{a}^{k}+p_{a}^{k}\right] \leq 0 & \forall k \in \mathcal{K}, \\
& \sum_{a \in \mathcal{A}} x_{a}^{k} \leq 1 & \forall k \in \mathcal{K}, \\
& p_{a}^{k}=T_{a} x_{a}^{k} & \forall k \in \mathcal{K}, \forall a \in \mathcal{A}, \\
& x_{a}^{k} \in\{0,1\} & \forall k \in \mathcal{K}, \forall a \in \mathcal{A}, \\
& T_{a} \geq 0 & \forall a \in \mathcal{A} .
\end{array}
$$

The (MP) we choose is composed of the objective function (4.7a) and constraints (4.7b), (4.7d) and (4.7f). Our sets $\mathcal{X}_{a}$, defining the feasible region of the subproblem, are represented by points satisfying constraints $4.7 \mathrm{e}),(4.7 \mathrm{f})$ and $(4.7 \mathrm{~g})$, which are separable for each path $a \in \mathcal{A}$. Moreover we add to the subproblem the following set of constraints:

$$
p_{a}^{k} \leq M_{a}^{k} x_{a}^{k} \quad \forall k \in \mathcal{K}, \forall a \in \mathcal{A} .
$$

It is straightforward to verify that these constraints are valid, as they derive from the linearisation of constraints (4.7e). Moreover, if we aggregate them summing over all paths $a \in \mathcal{A}$, we obtain constraints (4.7c), such that by including constraints 4.8 ) in the subproblem we verify constraints $4.7 \mathrm{c}$ ) and we can omit them in the (MP).

We represent a feasible solution of the subproblem by a point $\left(x_{a}^{k}, T_{a}, p_{a}^{k}\right)^{j}$, for $j \in \mathcal{J}$ where $\mathcal{J}$ is the space of feasible points for each $a, k$, and we associate to it a variable $\lambda_{a}^{j} \geq 0$. Note that sets $\mathcal{X}_{a}$ are not discrete as in the general example introduced in the previous section, as we have binary variables $x_{a}^{k}$ together with continuous variables $T_{a}$ and $p_{a}^{k}$. Let us denote $\left(x_{a}^{k, j}, T_{a}^{j}, p_{a}^{k, j}\right)$ as the $j^{t h}$ point of a set $\mathcal{X}_{a}$. For each $a \in \mathcal{A}$ we have:

$$
\left(\begin{array}{c}
x_{a}^{k} \\
T_{a} \\
p_{a}^{k}
\end{array}\right)=\sum_{j \in \mathcal{J}} \lambda_{a}^{j}\left(\begin{array}{c}
x_{a}^{k, j} \\
T_{a}^{j} \\
p_{a}^{k, j}
\end{array}\right)=\lambda_{a}^{1}\left(\begin{array}{c}
x_{a}^{k, 1} \\
T_{a}^{1} \\
p_{a}^{k, 1}
\end{array}\right)+\lambda_{a}^{2}\left(\begin{array}{c}
x_{a}^{k, 2} \\
T_{a}^{2} \\
p_{a}^{k, 2}
\end{array}\right)+\ldots+\lambda_{a}^{|\mathcal{J}|}\left(\begin{array}{c}
x_{a}^{k,|\mathcal{J}|} \\
T_{a}^{|\mathcal{J}|} \\
p_{a}^{k,|\mathcal{J}|}
\end{array}\right)
$$

We also impose $\sum_{j \in \mathcal{J}} \lambda_{a}^{j}=1$ for each $a \in \mathcal{A}$ (convexity constraint). Note that as sets $\mathcal{X}_{a}$ are not discrete $\lambda_{a}^{j}$ variables do not need to be binary. However, the integrality of the original variables $x_{a}^{k}$ is guaranteed by keeping constraints $4.7 \mathrm{f}$ ) also in the (MP).

We therefore express the (MP) using the convex combination of the feasible points of sets $\mathcal{X}_{a}$ : 
(MP)

$$
\begin{array}{llr}
\max _{\lambda} & \sum_{a \in \mathcal{A}} \sum_{k \in \mathcal{K}} \sum_{j \in \mathcal{J}} \lambda_{a}^{j} \eta^{k} p_{a}^{k, j}, & \\
\text { s.t. } & \sum_{a \in \mathcal{A}} \sum_{j \in \mathcal{J}}\left[\left(c_{a}^{k}-c_{o d}^{k}\right) \lambda_{a}^{j} x_{a}^{k, j}+\lambda_{a}^{j} p_{a}^{k, j}\right]-\sum_{j \in \mathcal{J}} \lambda_{b}^{j} T_{b}^{j} \leq c_{b}^{k}-c_{o d}^{k} & \forall k \in \mathcal{K}, \forall b \in \mathcal{A}, \\
& \sum_{a \in \mathcal{A}} \sum_{j \in \mathcal{J}} \lambda_{a}^{j} x_{a}^{k, j} \leq 1 & \forall k \in \mathcal{K}, \\
& \sum_{j \in \mathcal{J}} \lambda_{a}^{j}=1 & \forall a \in \mathcal{A}, \\
& \sum_{j \in \mathcal{J}} \lambda_{a}^{j} x_{a}^{k, j} \in\{0,1\} & \forall a \in \mathcal{A}, \forall k \in \mathcal{K}, \\
& \lambda_{a}^{j} \geq 0 & \forall j \in \mathcal{J}, \forall a \in \mathcal{A},
\end{array}
$$

where variables are $\lambda_{a}^{j}$, such that the problem is linear but with a huge set of variables.

As we want to solve the (MP) within a Branch-and-Bound framework, first of all we solve the linear relaxation of (MP), eliminating constraints $4.10 \mathrm{e}$. In the remaining of the chapter we discuss how to solve the linear relaxation of (MP) (for simplicity where there is no ambiguity we call it (MP)), whilst in Chapter 5 integer solutions are discussed.

Since the number of variables $\lambda_{a}^{j}$ is exponential, we use column generation to solve (MP), solving it on a subset of variables and using a pricing subproblem (SP) to improve the solution. We call (HPDW) the model obtained from (HPNL) with the Dantzig-Wolfe reformulation, and solve it with the following column generation algorithm.

Algorithm 4.3.1 (Column generation algorithm to solve the linear relaxation of (MP)).

Step 1: Initialise the linear relaxation of the restricted (MP) with a set of columns (see Section 4.6.

Step 2: Solve the linear relaxation of the restricted (MP).

Step 3: Extract dual variables values from the linear relaxation of the current restricted (MP) and calculate the maximum reduced price for each path $a$ (solve $\left(\mathrm{SP}_{a}\right)$ as described below).

Step 4: If for all paths $a$ the optimal reduced price is zero or negative, then STOP (the solution found for the linear relaxation of the restricted (MP) is optimal for (MP)). If not, for all paths $a$ for which the maximum reduced price is positive, add the corresponding optimal column (i.e. the optimal solution of $\left.(\mathrm{SP})_{a}\right)$ to the restricted (MP) and go back to Step 2 . 


\subsubsection{Subproblem}

As described in the general methodology (see Section 4.2), a pricing Subproblem (SP) is used to verify whether the (MP) on a subset of variables is optimal, i.e. if all possible variables have a non positive reduced price, and if not to search for new variables/ columns to add to improve the (MP) solution. Solutions of (SP) verify the constraints of the original problem (HPNL) that were not included in (MP): $4.7 \mathrm{c}$, , $4.7 \mathrm{e}$ ) and $4.7 \mathrm{~g}$ ), plus constraints (4.7f) that we include in both (MP) and (SP).

As we introduced above, constraints $4.7 \mathrm{c}$ ) are satisfied by including in (SP) the following set of constraints:

$$
p_{a}^{k}-M_{a}^{k} x_{a}^{k} \leq 0 \quad \forall k \in \mathcal{K}, \forall a \in \mathcal{A},
$$

where $M_{a}^{k}=\max \left\{0, c_{o d}^{k}-c_{a}^{k}\right\}$ as described in Section 1.5.7. These constraints, combined with constraints $4.7 \mathrm{e}$, reduce the domain of variables $T_{a}$ and $p_{a}^{k}$ : if $x_{a}^{k}=0$ these constraints impose $p_{a}^{k}=0$, whereas if $x_{a}^{k}=1$ they become $T_{a} \leq M_{a}^{k}$, which is a valid upper bound on the toll if commodity $k$ uses path $a$ (see Section 1.5.7). Furthermore, they guarantee that in the subproblem there are no solutions with $x_{a}^{k}=1$ if the corresponding $T_{a}>0$ and $M_{a}^{k}=0$.

We define the dual variables associated to constraints of (MP) as follows:

- variables $\delta_{b}^{k} \geq 0$ are associated to constraints $4.10 \mathrm{~b}$, one for each $b \in \mathcal{A}$ and $k \in \mathcal{K}$;

- variables $\gamma^{k} \geq 0$ are associated to constraints 4.10c), one for each $k \in \mathcal{K}$;

- variables $\mu_{a} \gtreqless 0$ are associated to constraints $4.10 \mathrm{~d}$ ), one for each $a \in \mathcal{A}$.

Proposition 4.3.1 ((SP) is easy). The Subproblem (SP) used to solve (MP) (Equations 4.10a) to $(4.10 f)$ ) is separable for each path $a \in \mathcal{A}$ and each $(S P)_{a}$ achieves its optimal value in $\left\{M_{a}^{k}\right.$ : $k \in \mathcal{K}\} \cup\{0\}$, so only $|\mathcal{K}|+1$ values of $T_{a}$ must be evaluated for solving $\left(S P_{a}\right)$.

Proof. The reduced price of a variable $\lambda_{a}^{j}$ of the (MP) can be expressed as follows:

$$
R P_{a}=\sum_{k \in \mathcal{K}} \eta^{k} p_{a}^{k}-\left\{\sum_{k \in \mathcal{K}} \sum_{b \in \mathcal{A}} \delta_{b}^{k}\left[\left(c_{a}^{k}-c_{o d}^{k}\right) x_{a}^{k}+p_{a}^{k}\right]-\sum_{k \in \mathcal{K}} \delta_{a}^{k} T_{a}+\sum_{k \in \mathcal{K}} x_{a}^{k} \gamma^{k}+\mu_{a}\right\} .
$$

The reduced price $R P_{a}$ is defined for each path $a$ and we noticed that (SP) constraints are separable for each path $a$, meaning that we can solve a smaller subproblem for each path $a \in \mathcal{A}$, which can be formulated as follows: 


$$
\begin{array}{rlr}
\max _{T, x, p} & \sum_{k \in \mathcal{K}} \eta^{k} p_{a}^{k}-\left[\sum_{k \in \mathcal{K}} \sum_{b \in \mathcal{A}} \delta_{b}^{k}\left(p_{a}^{k}-M_{a}^{k} x_{a}^{k}\right)-\sum_{k \in \mathcal{K}} \delta_{a}^{k} T_{a}+\sum_{k \in \mathcal{K}} x_{a}^{k} \gamma^{k}+\mu_{a}\right] \\
\text { s.t } & p_{a}^{k}-M_{a}^{k} x_{a}^{k} \leq 0 & \forall k \in \mathcal{K} \\
& p_{a}^{k}=T_{a} x_{a}^{k} & \forall k \in \mathcal{K} \\
& x_{a}^{k} \in\{0,1\} & \forall k \in \mathcal{K} \\
& T_{a} \geq 0
\end{array}
$$

This problem is non-linear due to (4.13c), but it is easy to solve, as we are solving a problem for a single path. We notice that if the value of $x_{a}^{k}$ is fixed for all $k \in \mathcal{K}$, then the objective function becomes linear in $T_{a}$, and, as for the single toll path case (see Section 1.4.2, the commodities path choice may change only at toll values $T_{a} \in\left\{M_{a}^{k}\right.$ : $k \in \mathcal{K}\} \cup\{0\}$. Any other toll value can be increased without changing the commodities path choice and therefore increasing the objective function value. We then consider only these breakpoint values for $T_{a}$ and we deduce the assignment values of commodities to toll paths $x_{a}^{k}$ with the following procedure.

We can rewrite the reduced price, indicating explicitly the coefficient of $x_{a}^{k}$ variables (remember that $p_{a}^{k}=T_{a} x_{a}^{k}$ ):

$$
R P_{a}=\sum_{k \in \mathcal{K}} x_{a}^{k}\left[T_{a}\left(\eta^{k}-\sum_{b \in \mathcal{A}} \delta_{b}^{k}\right)+\sum_{b \in \mathcal{A}} M_{a}^{k} \delta_{b}^{k}-\gamma^{k}\right]+\sum_{k \in \mathcal{K}} \delta_{a}^{k} T_{a}-\mu_{a}
$$

Denote as $\pi_{a}^{k}$ the coefficient associated to $x_{a}^{k}$ in the reduced price, $\forall a \in \mathcal{A}$ and $\forall k \in \mathcal{K}$ :

$$
\pi_{a}^{k}=T_{a}\left(\eta^{k}-\sum_{b \in \mathcal{A}} \delta_{b}^{k}\right)+\sum_{b \in \mathcal{A}} M_{a}^{k} \delta_{b}^{k}-\gamma^{k}
$$

For each $T_{a} \in\left\{M_{a}^{k}: k \in \mathcal{K}\right\} \cup\{0\}$, we calculate the value of $\pi_{a}^{k}$ using Equation (4.15) and the assignment of commodities to paths that maximises the reduced price is as follows:

$$
x_{a}^{k}= \begin{cases}1 & \text { if } M_{a}^{k} \geq T_{a} \text { and } \pi_{a}^{k}>0 \\ 0 & \text { otherwise }\end{cases}
$$

and then we calculate the corresponding reduced price: 


$$
R P_{a}\left(T_{a}\right)=\sum_{k \in \mathcal{K}} x_{a}^{k} \pi_{a}^{k}+\sum_{k \in \mathcal{K}} \delta_{a}^{k} T_{a}-\mu_{a}
$$

We choose the optimal value $T_{a}^{*}=\arg \max _{T_{a}}\left\{R P_{a}\left(T_{a}\right): T_{a} \in\left\{M_{a}^{k}: k \in \mathcal{K}\right\} \cup\{0\}\right\}$ and the corresponding assignment values.

Each (SP) $)_{a}$ is solved evaluating $|\mathcal{K}|+1$ values, such that to solve (SP) we need to evaluate $O(|\mathcal{A}||\mathcal{K}|)$ values.

For each path $a$ the number of total columns is bounded by all the possible values for the toll, which is $O(|\mathcal{K}|)$, multiplied by all the possible assignment matrices of the commodities, which is $2^{|\mathcal{K}|}$. The total number of columns is therefore $O\left(|\mathcal{A}||\mathcal{K}| 2^{|\mathcal{K}|}\right)$.

\subsubsection{Dual bound for column generation}

As introduced in Section 4.2, Equation (4.6), at each iteration of the column generation algorithm we can calculate a dual upper bound for the optimal value of the linear relaxation of (MP) as follows:

$$
U B=\sum_{k \in \mathcal{K}} \gamma^{k}+\sum_{a \in \mathcal{A}} \mu_{a}-\sum_{b \in \mathcal{A}} \sum_{k \in \mathcal{K}} M_{b}^{k} \delta_{b}^{k}+\sum_{a \in \mathcal{A}} \mathrm{RP}_{a}
$$

where $\mathrm{RP}_{a}$ is the reduced price associated to path $a$, calculated with equation 4.14.

\subsubsection{Description of instances for numerical experiments}

Numerical experiments presented in Chapters 4 and 5 of this thesis have been run on SCIP 3.0.1 framework (Achterberg, 2009), on a server with 16 Intel XEON CPUs at 2.27 $\mathrm{GHz}$ and with $16.00 \mathrm{~Gb}$ of Ram running Linux, using Cplex 12.5 as the solver for linear programming. Some different types of instances have been considered for numerical tests, which are described in the following part of the section. Reported results are on average of 10 instances for each type and size.

\section{Complete and partial graph instances}

We consider 20, 56 and 90 commodities and toll paths. These instances do not correspond to any underlying network, in the sense that we generate directly costs and demand data for each toll path and commodity.

Fixed costs on toll paths are randomly set between 1 and 20, and demand for commodities is randomly selected between 1 and 10 . Complete network means that toll free path costs are randomly generated between 20 and 30 (and so are always bigger than fixed costs of toll paths), such that all commodities could use all toll paths, whilst for a partial network we randomly set toll free path costs between 10 and 30, meaning that some toll paths will never be interesting for some commodities. 
We notice from numerical tests that these instances, especially the partial graphs, are quite difficult to solve (they have a large gap at the root node).

\section{Shioda et al. instances}

These instances have been introduced in Shioda et al. (2011) to test product pricing problems, and later used by Heilporn et al. (2011) to compare the NPP formulation to solve product pricing problems to the product pricing formulation (see Section 1.6).

Shioda et al. (2011) address randomly generated instances with 40,60 or 80 customers (commodities) and 20, 40 or 60 products (toll paths). They randomly generate the demand value for each customer between 500 and 799, and the reservation price for each product and each customer (which is for the NPP the $M_{a}^{k}$ value, equal to $\left(c_{o d}^{k}-c_{a}^{k}\right)$ ) between 512 and 1023. The toll path network is complete in the sense that all commodities might use each toll path, having a positive $M$ value.

We notice from numerical tests that these instances, despite having a similar size as the previous ones, are easier to solve (they have a smaller gap at the root node).

\section{A1 instances}

In order to test our algorithms on more realistic instances with an underlying network, we generated a set of instances on the Italian "A1" highway, which connects Milano to Napoli going from north to south of the country. To construct the toll paths network we considered the principal entry and exit nodes of the highway, which are represented by the following fourteen cities: Milano, Lodi, Piacenza, Parma, Reggio Emilia, Modena, Bologna, Firenze, Arezzo, Orvieto, Roma, Frosinone, Caserta and Napoli. The real length of the highway's arcs is publicly available on-line (see A1data). We considered highway's subnetworks with 7,11 and 14 subsequent entry and exit nodes, which correspond to 21, 55 and 91 toll paths respectively, as $n$ nodes provide $n(n-1) / 2$ pairs of entry/exit nodes (as all parameters are symmetrical we consider only the highway network in one direction).

Commodities are chosen between pairs of cities in a set composed by the cities on the highway nodes plus eight additional cities distributed in the surroundings of the highway (Brescia, Verona, Genova, Livorno, Perugia, L'Aquila, Latina and Salerno), for a total of 22 cities. We construct instances with 7,11 and 14 cities randomly chosen in the set, corresponding to 21,55 and 91 commodities respectively (a commodity is a pair of origin/destination cities). The demand for each commodity has been calculated with a simple gravitational model (see Rosenberg):

$$
\operatorname{demand}(\operatorname{city} 1-\operatorname{city} 2)=\frac{\text { population }(\operatorname{city} 1) * \operatorname{population}(\operatorname{city} 2)}{\operatorname{distance}^{2}(\operatorname{city} 1-\operatorname{city} 2)}
$$

where the population numbers have been obtained from publicly available on-line data and the distance between cities from GoogleMaps. All the distances between each city 
and each entry/exit node of the highway have also been obtained from GoogleMaps (using the option "no highways and no tolls").

Finally, all distances on non highway arcs have been multiplied by a random value between 1.5 and 1.7, to take into account the speed difference when travelling on the highway or not (if we consider $120 \mathrm{~km} / \mathrm{h}$ as the average speed on the highway and 75 $\mathrm{km} / \mathrm{h}$ on other routes we obtain a factor of 1.6).

Numerical results show that this class of instances is easier to solve compared to the first class, as they require less solution time and they have a quite small gap at the root node.

\subsubsection{Numerical results}

In Table 4.1 we report results about our column generation algorithm to solve the linear relaxation of (HPDW). We report the time in seconds to find the optimal relaxed solution of (HPDW), and separately to solve the (MP) and the (SP). Furthermore, we report the number of columns generated during the solving and the number of iterations of the algorithm, which is the number of times the (MP) and (SP) are solved.

We notice that, for the first three class of instances and when the solution time is significant, the algorithm spends more time in solving the (MP), whilst the (SP) is quickly solved. On the contrary for A1 instances the algorithm has a longer (SP) solution time compared to its (MP) solution time. Note that it is (MP) to be solved faster compared to the other classes of instances: this difference might be due to the fact that are a lot of zero values in the $M_{a}^{k}$ matrices of A1 instances, as many toll paths are not interesting for many commodities (in practice they consist in too long detours). These zero $M_{a}^{k}$ values result in forbidding the assignment of commodities to paths and therefore the solving of the (MP) is quite simple.

The number of generated columns is increasing with the size of instances, which is expected, but it does not become too big. For instances with 20 commodities we generate the $10^{-5} \%$ of the corresponding total number of columns, the $10^{-16 \%}$ for 40 56 commodities, the $10^{-22} \%$ for 80 commodities and the $10^{-26 \%}$ for 90 commodities. Moreover, less columns are needed to solve A1 instances with respect to complete and partial graph instances, despite having a similar size. This is again due to having many zero $M_{a}^{k}$ values, such that the (SP) has a smaller solution space.

For each class of instances with the same number of commodities, the number of iterations decreases when the number of toll paths increases. The number of iterations also provides an average time needed to solve each (MP) and (SP): for big instances (with 90 commodities and 90 toll paths) each (MP) takes around 1.5 seconds to be solved, and each (SP) 0.5 seconds. For big A1 instances each (MP) takes around 0.15 seconds to be solved, and each (SP) 0.5 seconds as for the other classes of instances. Furthermore, Shioda et al. instances need more iterations and columns to be solved, which results in larger solution time, whilst A1 instances have a smaller solution time, 


\begin{tabular}{|c|c|c|c|c|c|c|c|c|c|c|}
\hline \multirow{2}{*}{$\begin{array}{l}\text { Complete } \\
\text { graph }\end{array}$} & & \multicolumn{3}{|c|}{20 commodities } & \multicolumn{3}{|c|}{56 commodities } & \multicolumn{3}{|c|}{90 commodities } \\
\hline & & $20 \mathrm{a}$ & $56 \mathrm{a}$ & $90 \mathrm{a}$ & $20 \mathrm{a}$ & $56 \mathrm{a}$ & $90 \mathrm{a}$ & $20 \mathrm{a}$ & $56 \mathrm{a}$ & $90 \mathrm{a}$ \\
\hline \multirow[t]{3}{*}{ (HPDW) } & Time & 0.199 & 0.726 & 1.57 & 4.13 & 12.7 & 22.3 & 25.84 & 62.26 & 108.99 \\
\hline & Cols & 335 & 655 & 924 & 1141 & 1765 & 2227 & 2103 & 3034 & 3749 \\
\hline & Iters & 21 & 15 & 14 & 63 & 37 & 29 & 111 & 61 & 47 \\
\hline (MP) & Time & 0.072 & 0.206 & 0.41 & 2.926 & 8.197 & 13.36 & 22.05 & 48.84 & 81.3 \\
\hline$(\mathrm{SP})$ & Time & 0.116 & 0.509 & 1.135 & 1.159 & 4.401 & 8.863 & 3.669 & 13.27 & 27.46 \\
\hline \multirow{2}{*}{\multicolumn{2}{|c|}{$\begin{array}{l}\text { Partial } \\
\text { graph }\end{array}$}} & \multicolumn{3}{|c|}{20 commodities } & \multicolumn{3}{|c|}{56 commodities } & \multicolumn{3}{|c|}{90 commodities } \\
\hline & & $20 \mathrm{a}$ & $56 \mathrm{a}$ & $90 \mathrm{a}$ & $20 \mathrm{a}$ & $56 \mathrm{a}$ & $90 \mathrm{a}$ & $20 \mathrm{a}$ & $56 \mathrm{a}$ & $90 \mathrm{a}$ \\
\hline \multirow[t]{3}{*}{ (HPDW) } & Time & 0.171 & 0.687 & 1.443 & 3.026 & 10.99 & 21.23 & 18.54 & 55.71 & 102.87 \\
\hline & Cols & 301 & 625 & 891 & 974 & 1699 & 2199 & 1853 & 2926 & 3747 \\
\hline & Iters & 19 & 15 & 13 & 55 & 35 & 29 & 100 & 58 & 47 \\
\hline$(\mathrm{MP})$ & Time & 0.063 & 0.204 & 0.387 & 1.832 & 6.81 & 12.63 & 15.27 & 42.99 & 76.26 \\
\hline (SP) & Time & 0.101 & 0.474 & 1.04 & 1.16 & 4.135 & 8.52 & 3.176 & 12.56 & 26.4 \\
\hline \multirow{2}{*}{\multicolumn{2}{|c|}{$\begin{array}{l}\text { Shioda et al. } \\
\text { instances }\end{array}$}} & \multicolumn{3}{|c|}{40 commodities } & \multicolumn{3}{|c|}{60 commodities } & \multicolumn{3}{|c|}{80 commodities } \\
\hline & & $20 \mathrm{a}$ & $40 \mathrm{a}$ & $60 \mathrm{a}$ & $20 \mathrm{a}$ & $40 \mathrm{a}$ & $60 \mathrm{a}$ & $20 \mathrm{a}$ & $40 \mathrm{a}$ & $60 \mathrm{a}$ \\
\hline \multirow[t]{3}{*}{ (HPDW) } & Time & 5.51 & 7.79 & 11.39 & 43.12 & 47.04 & 56.83 & 198.38 & 221.28 & 260.09 \\
\hline & Cols & 1413 & 1714 & 2015 & 2721 & 3046 & 3506 & 4586 & 5130 & 5645 \\
\hline & Iters & 82 & 50 & 40 & 165 & 91 & 69 & 277 & 153 & 110 \\
\hline (MP) & Time & 4.37 & 5.37 & 7.26 & 39.76 & 40.27 & 45.13 & 190.54 & 205.3 & 233 \\
\hline$(\mathrm{SP})$ & Time & 1.1 & 2.37 & 4.07 & 3.23 & 6.62 & 11.53 & 7.49 & 15.66 & 26.71 \\
\hline \multirow{2}{*}{\multicolumn{2}{|c|}{$\begin{array}{l}\text { A1 } \\
\text { instances }\end{array}$}} & \multicolumn{3}{|c|}{21 commodities } & \multicolumn{3}{|c|}{55 commodities } & \multicolumn{3}{|c|}{91 commodities } \\
\hline & & $21 \mathrm{a}$ & $55 \mathrm{a}$ & $91 \mathrm{a}$ & $21 \mathrm{a}$ & $55 \mathrm{a}$ & $91 \mathrm{a}$ & $21 \mathrm{a}$ & $55 \mathrm{a}$ & $91 \mathrm{a}$ \\
\hline \multirow[t]{3}{*}{ (HPDW) } & Time & 0.07 & 0.22 & 0.46 & 0.48 & 1.69 & 3.85 & 3.14 & 8.63 & 16.45 \\
\hline & Cols & 137 & 333 & 460 & 347 & 572 & 863 & 727 & 1185 & 1527 \\
\hline & Iters & 11 & 12 & 11 & 30 & 21 & 19 & 59 & 39 & 29 \\
\hline (MP) & Time & 0.02 & 0.04 & 0.07 & 0.16 & 0.34 & 0.64 & 1.67 & 2.69 & 4.37 \\
\hline$(\mathrm{SP})$ & Time & 0.04 & 0.17 & 0.40 & 0.32 & 1.35 & 3.21 & 1.47 & 5.94 & 12.08 \\
\hline
\end{tabular}

Table 4.1: Numerical results on the column generation algorithm for the linear relaxation of (HPDW)

despite the similar size in number of commodities and toll paths.

In Figure 4.1 we plot the evolution of the primal and dual bound during the solving of one instance with the column generation algorithm. The primal lower bound represents the solution of the restricted (MP) at each iteration, with the current (non optimal) subset of columns. The dual upper bound is calculated as described in Section 4.3.3 and its values at each iteration are marked with a "I" sign. We notice that, as expected, the primal bound is always increasing, whilst the dual bound has an oscillatory behaviour, especially for the Shioda et al. instance (Figure 4.1(b)).

There is also a "tailing-off" effect: when close to optimality improvements are very small, and often we already have the optimal solution but the proof of optimality takes a lot of time. Therefore there is a long "tail" in the solution process, as we can see from graphs of Figure 4.1. Numerical instability can also contribute to a bad behaviour of the stopping criterion of the column generation algorithm.

Oscillatory behaviour and tailing-off effect have been revealed in experiments from 
the literature and can be limited with the additional stopping criterion presented in Section 4.8 and with stabilisation techniques that we discuss in Section 4.9 . More details and references can be found in Lübbecke and Desrosiers (2005).

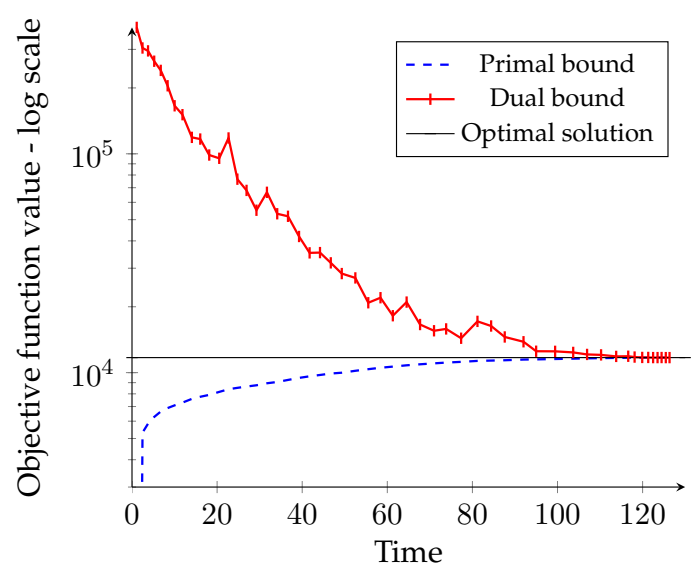

(a) 90k 90a complete graph instance

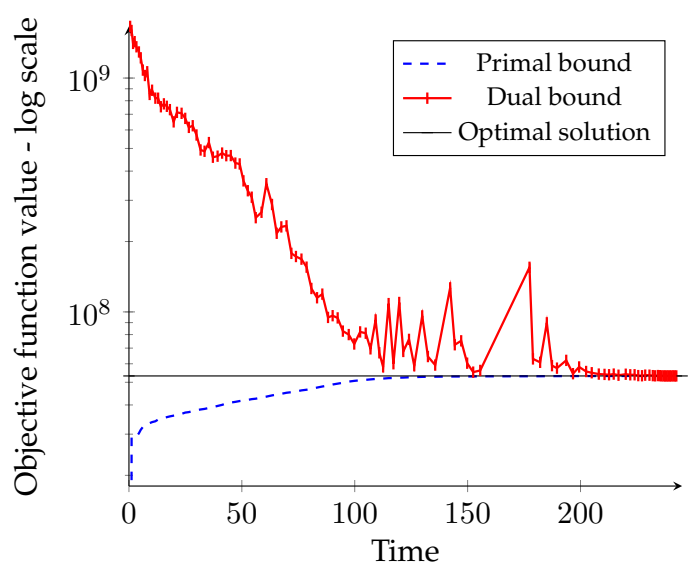

(b) $80 \mathrm{k} 60 \mathrm{a}$ Shioda et al. instance

Figure 4.1: Primal and dual bound evolution while solving the linear relaxation of (HPDW) with column generation

\subsection{An extended formulation}

The development of the Dantzig-Wolfe reformulation gives us a better understanding on the structure of the problem and allows us to develop an extended compact formulation for the NPP with connected toll arcs, which we present in this section.

In the Dantzig-Wolfe reformulation tolls are expressed as $T_{a}=\sum_{j} \lambda_{a}^{j} T_{a}^{j}$, for each path $a \in \mathcal{A}$. When solving (SP) we saw that $T_{a}^{j}$ takes values in $\left\{M_{a}^{k}: k \in \mathcal{K}\right\} \cup\{0\}$ (see Section 4.3.2). Therefore $T_{a}$ can be expressed as a convex combination of $M_{a}^{k}$ values.

For each $a \in \mathcal{A}$, we order the commodities according to their non decreasing $M_{a}^{k}$ values, and rename those values $m_{a}^{l}$. More precisely, we obtain a list of values $m_{a}^{l}$, $l=0, \ldots,|\mathcal{K}|+1$, such that $m_{a}^{l} \leq m_{a}^{l+1}$ and $r_{a}(k)=l$ if $m_{a}^{l}=M_{a}^{k}$. We define $m_{a}^{0}=0$ and $m_{a}^{|\mathcal{K}|+1}=N_{a}\left(=\max _{k \in \mathcal{K}} M_{a}^{k}\right)$, for each $a \in \mathcal{A}$.

A toll $T_{a}$ lies in an interval $\left[m_{a}^{l}, m_{a}^{l+1}\right]$, as represented in Figure 4.2 .

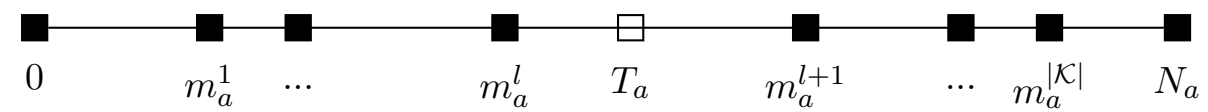

Figure 4.2: Representation of $T_{a}$ in the interval of two subsequent $m_{a}^{l}$ values

Let us define two sets of variables, one set of binary variables $z_{a}^{l}$ to indicate in which interval $T_{a}$ lies, and one set of continuous variables $u_{a}^{l}$ to indicate the position of $T_{a}$ in 
such an interval, for each $a \in \mathcal{A}$ and $l \in \mathcal{K}$ :

$$
z_{a}^{l}=\left\{\begin{array}{ll}
1 & \text { if } T_{a} \in\left[m_{a}^{l}, m_{a}^{l+1}\right] \\
0 & \text { otherwise }
\end{array} \quad \text { and } \quad 0 \leq u_{a}^{l} \leq 1 .\right.
$$

Tolls $T_{a}$ can be expressed with these variables as follows, for each $a \in \mathcal{A}$ :

$$
T_{a}=\sum_{l \in \mathcal{K}} m_{a}^{l} z_{a}^{l}+\sum_{l \in \mathcal{K}} u_{a}^{l}\left(m_{a}^{l+1}-m_{a}^{l}\right)
$$

with the following constraints:

$$
\begin{array}{lr}
\sum_{l \in \mathcal{K}} z_{a}^{l}=1 & \forall a \in \mathcal{A}, \\
0 \leq u_{a}^{l} \leq z_{a}^{l} & \forall a \in \mathcal{A}, \forall l \in \mathcal{K} .
\end{array}
$$

Constraints (4.22a) guarantee that we choose only one interval $\left[m_{a}^{l}, m_{a}^{l+1}\right]$ for each $T_{a}$ to lie in, and constraints $4.22 \mathrm{~b}$ guarantee that an interval position variable $u_{a}^{l}$ is positive only if the corresponding interval has been chosen, i.e. the corresponding $z_{a}^{l}$ is not zero.

To express assignment of commodities to paths, i.e. original variables $x_{a}^{k}$, we define the following new set of variables, for each $a \in \mathcal{A}$ and $k, l \in \mathcal{K} \times \mathcal{K}$ :

$$
w_{a}^{k l}= \begin{cases}1 & \text { if } x_{a}^{k}=1 \text { and } T_{a} \in\left[m_{a}^{l}, m_{a}^{l+1}\right] \\ 0 & \text { otherwise }\end{cases}
$$

Note that a variable $w_{a}^{k l}$ exists only if $T_{a} \leq M_{a}^{k}$, i.e. if $l<r_{a}(k)$.

Assignment variables $x_{a}^{k}$ can be therefore expressed as follows, $\forall a \in \mathcal{A}, \forall k \in \mathcal{K}$ :

$$
x_{a}^{k}=\sum_{l \in \mathcal{K}: l<r_{a}(k)} w_{a}^{k l}
$$

with the following constraints:

$$
\begin{array}{lr}
\sum_{l \in \mathcal{K}: l<r_{a}(k)} w_{a}^{k l} \leq 1 & \forall a \in \mathcal{A}, \forall k \in \mathcal{K}, \\
w_{a}^{k l} \leq z_{a}^{l} & \forall a \in \mathcal{A}, \forall k \in \mathcal{K}, \forall l \in \mathcal{K}: l<r_{a}(k) .
\end{array}
$$

Constraints $4.25 \mathrm{~b}$ ) are valid as $z_{a}^{l}=1$ only if $T_{a} \in\left[m_{a}^{l}, m_{a}^{l+1}\right]$.

Finally, we introduce one more set of continuous variables $v_{a}^{k l}$ as follows:

$$
v_{a}^{k l}= \begin{cases}u_{a}^{l} & \text { if } w_{a}^{k l}=1 \\ 0 & \text { otherwise }\end{cases}
$$


and variables $p_{a}^{k}$ can therefore be expressed as follows, for each $a \in \mathcal{A}$ and $k \in \mathcal{K}$ :

$$
p_{a}^{k}=\sum_{l \in \mathcal{K}: l<r_{a}(k)} m_{a}^{l} w_{a}^{k l}+\sum_{l \in \mathcal{K}: l<r_{a}(k)} v_{a}^{k l}\left(m_{a}^{l+1}-m_{a}^{l}\right),
$$

with the following constraints:

$$
\begin{aligned}
& v_{a}^{k l} \leq w_{a}^{k l} \quad \forall a \in \mathcal{A}, \forall k \in \mathcal{K}, \forall l \in \mathcal{K}: l<r_{a}(k), \\
& v_{a}^{k l} \leq u_{a}^{l} \quad \forall a \in \mathcal{A}, \forall k \in \mathcal{K}, \forall l \in \mathcal{K}: l<r_{a}(k), \\
& v_{a}^{k l} \geq u_{a}^{l}-\left(1-w_{a}^{k l}\right) \quad \forall a \in \mathcal{A}, \forall k \in \mathcal{K}, \forall l \in \mathcal{K}: l<r_{a}(k), \\
& v_{a}^{k l} \geq 0 \quad \forall a \in \mathcal{A}, \forall k \in \mathcal{K}, \forall l \in \mathcal{K}: l<r_{a}(k) .
\end{aligned}
$$

Note that constraints $4.28 \mathrm{c}$ ) can be strengthened by replacing them with the following set of valid inequalities:

$$
v_{a}^{k l} \geq u_{a}^{l}+w_{a}^{k l}-z_{a}^{l} \quad \forall a \in \mathcal{A}, \forall k \in \mathcal{K}, \forall l \in \mathcal{K}: l<r_{a}(k) .
$$

Inequalities 4.29) are valid as we have:

- if $z_{a}^{l}=0$ then $w_{a}^{k l}=0$ by constraints $4.25 \mathrm{~b}$ ) and $u_{a}^{l}=0$ by constraints $4.22 \mathrm{~b}$, and therefore the constraints become $v_{a}^{k l} \geq 0$ which is valid by the definition of variables $v_{a}^{k l}$ of Equation 4.26;

- if $z_{a}^{l}=1$ and $w_{a}^{k l}=1$, then the constraints become $v_{a}^{k l} \geq u_{a}^{l}$ which combined with constraints $4.28 \mathrm{~b}$ implies $v_{a}^{k l}=u_{a}^{l}$ complying the definition of variables $v_{a}^{k l}$ of Equation (4.26);

- if $z_{a}^{l}=1$ and $w_{a}^{k l}=0$ then the constraints become $v_{a}^{k l} \geq u_{a}^{l}-1$ which is always verified as $u_{a}^{l} \leq 1$ by constraints $4.22 \mathrm{~b}$.

Constraints 4.29) are not weaker than constraints 4.28c as $z_{a}^{l} \leq 1$ by the definition of $z_{a}^{l}$ variables of Equation 4.20.

Moreover, in Section 4.3.1 we introduced the set of inequalities 4.8), showing that they are valid and that they satisfy constraints $4.7 \mathrm{c}$ ) of (HPNL).

We now reformulate the NPP with connected toll arcs in this new space of variables $\left(z_{a}^{l}, u_{a}^{l}, w_{a}^{k l}, v_{a}^{k l}\right)$. We start from the (HPNL) formulation (Equations 4.7a to 4.7g), but with constraints (4.8) instead of (4.7c) and use the relation between the two spaces of variables just described, leading to the following linear Highway Problem Extended Formulation (HPEF): 
(HPEF)

$$
\begin{aligned}
& \max _{z, u, x, v} \sum_{a \in \mathcal{A}} \sum_{k \in \mathcal{K}} \eta^{k}\left[\sum_{l \in \mathcal{K}: l<r_{a}(k)} m_{a}^{l} w_{a}^{k l}+\sum_{l \in \mathcal{K}: l<r_{a}(k)} v_{a}^{k l}\left(m_{a}^{l+1}-m_{a}^{l}\right)\right], \\
& \text { s.t. } \quad \sum_{a \in \mathcal{A}}\left[\sum_{l \in \mathcal{K}: l<r_{a}(k)} w_{a}^{k l}\left(m_{a}^{l}-M_{a}^{k}\right)+\sum_{l \in \mathcal{K}: l<r_{a}(k)} v_{a}^{k l}\left(m_{a}^{l+1}-m_{a}^{l}\right)\right]+ \\
& -\sum_{l \in \mathcal{K}} m_{b}^{l} z_{b}^{l}-\sum_{l \in \mathcal{K}} u_{b}^{l}\left(m_{b}^{l+1}-m_{b}^{l}\right) \leq-M_{b}^{k} \\
& \sum_{l \in \mathcal{K}: l<r_{a}(k)} w_{a}^{k l}\left(m_{a}^{l}-M_{a}^{k}\right)+\sum_{l \in \mathcal{K}: l<r_{a}(k)} v_{a}^{k l}\left(m_{a}^{l+1}-m_{a}^{l}\right) \leq 0 \quad \forall k \in \mathcal{K}, \forall a \in \mathcal{A}, \\
& \sum_{a \in \mathcal{A}} \sum_{l \in \mathcal{K}: l<r_{a}(k)} w_{a}^{k l} \leq 1 \\
& \sum_{l \in \mathcal{K}} z_{a}^{l}=1 \\
& u_{a}^{l} \leq z_{a}^{l} \\
& w_{a}^{k l} \leq z_{a}^{l} \\
& v_{a}^{k l} \leq w_{a}^{k l} \\
& v_{a}^{k l} \leq u_{a}^{l} \\
& v_{a}^{k l} \geq u_{a}^{l}+w_{a}^{k l}-z_{a}^{l} \\
& z_{a}^{l}, x_{a}^{k l} \in\{0,1\} \\
& u_{a}^{l}, v_{a}^{k l} \geq 0 \\
& \forall a \in \mathcal{A}, \forall k, l\left(: l<r_{a}(k)\right) \in \mathcal{K} \times \mathcal{K}, \\
& \forall a \in \mathcal{A}, \forall k, l\left(: l<r_{a}(k)\right) \in \mathcal{K} \times \mathcal{K}, \\
& \forall a \in \mathcal{A}, \forall k, l\left(: l<r_{a}(k)\right) \in \mathcal{K} \times \mathcal{K}, \\
& \forall a \in \mathcal{A}, \forall k, l\left(: l<r_{a}(k)\right) \in \mathcal{K} \times \mathcal{K}, \\
& \forall a \in \mathcal{A}, \forall k, l\left(: l<r_{a}(k)\right) \in \mathcal{K} \times \mathcal{K}, \\
& \forall a \in \mathcal{A}, \forall k, l\left(: l<r_{a}(k)\right) \in \mathcal{K} \times \mathcal{K} .
\end{aligned}
$$

This extended formulation is linear and has a polynomial number of variables and constraints, but this number is of the order of $|\mathcal{A}| \times|\mathcal{K}| \times|\mathcal{K}|$, which may become quite large. For instance for 90 commodities and 90 toll paths we have 745200 variables and 1117980 constraints, whilst for (HPNL) we had 16290 variables and 16380 constraints.

Numerical results on this formulation are reported in Section 4.5.2, where we compare it to the Dantzig-Wolfe reformulation (HPDW) and the mixed-integer linear formulation (HPL). 


\subsection{Comparison between the formulations}

In this section we compare the different formulations we have for the NPP with connected toll arcs, analysing the optimal value of their linear relaxation compared to the optimal integer solution value.

\subsubsection{Theoretical comparison}

Consider the non linear model (HPNL) described by Equations 4.7a) to (4.7g) at the beginning of Section 4.3.1, the linear model (HPL) described by Equations (1.17a) to (1.17i) in Section 1.5.7 and the Dantzig-Wolfe reformulation (HPDW) described by Equations 4.10a to (4.10f and 4.13a to 4.13e in Section 4.3. We define as $F(*)$ the set of feasible solutions for a formulation $*$. Consider the linear relaxation of (HPL) and (HPDW) and note them as (HPL-LR) and (HPDW-LR) respectively.

Proposition 4.5.1. $F(H P D W-L R) \subseteq F(H P L-L R)$.

Proof. Let us describe $F$ (HPNL) $=\{x \in X: A x \leq b, x \in\{0,1\}\}$, where $X$ is the set of points satisfying constraints (4.7e), (4.7f) and (4.7g), $A x \leq b$ corresponds to constraints (4.7b), (4.7c) and (4.7d), and $x \in\{0,1\}$ to constraints (4.7f).

It is evident that $F(\mathrm{HPL})=F(\mathrm{HPNL})$ and we defined (HPDW) such that $F(\mathrm{HPDW})=$ $\{x \in \operatorname{conv}(X): A x \leq b, x \in\{0,1\}\}$.

We conclude that $F(\mathrm{HPDW}-\mathrm{LR}) \subseteq F(\mathrm{HPL}-\mathrm{LR})$ (see Geoffrion, 1974).

Therefore the linear relaxation of the Dantzig-Wolfe reformulation provides an upper bound for the optimal value of the integer problem as least as good as the linear relaxation of the linear model (HPL). The following example shows that in some cases it is strictly better.

Consider the network of Figure 4.3, where we have one toll path and three commodities (called $k_{1}, k_{2}, k_{3}$ ) with unit demand and toll free path cost of 10 . The fixed cost for using the toll path is equal to 1 for $k_{1}, 2$ for $k_{2}$ and 5 for $k_{3}$.

As there is only one toll path, we have only one subproblem in (HPDW) formulation. We then analyse all candidate values of the toll to construct the columns, i.e. $0, M^{k_{1}}=10-1=9, M^{k_{2}}=8$ and $M^{k_{3}}=5$. We consider one column for each of them: denote $j_{0}$ as the column with $T^{j_{0}}=0, j_{1}$ as the column with $T^{j_{1}}=9, j_{2}$ as the column with $T^{j_{2}}=8$, and $j_{3}$ as the column with $T^{j_{3}}=5$. We derive the optimal assignment of commodities to the path: for $j_{0}$ we obtain $x^{k_{1}, j_{0}}=x^{k_{2}, j_{0}}=x^{k_{3}, j_{0}}=1$ and a revenue of 0 , for $j_{1}$ we obtain $x^{k_{1}, j_{1}}=1, x^{k_{2}, j_{1}}=x^{k_{3}, j_{1}}=0$ and a revenue of 9 , for $j_{2}$ we obtain $x^{k_{1}, j_{2}}=x^{k_{2}, j_{2}}=1, x^{k_{3}, j_{2}}=0$ and a revenue of 16 , and for $j_{3}$ we obtain $x^{k_{1}, j_{3}}=x^{k_{2}, j_{3}}=x^{k_{3}, j_{3}}=1$ and a revenue of 15 . Note that we have $p^{k, j}=T^{j} x^{k, j}$. The optimal solution is therefore $\lambda^{j_{2}}=1$ and $\lambda^{j_{0}}=\lambda^{j_{1}}=\lambda^{j_{3}}=0$, with a value of 16 , and the optimal toll value is thus $T^{j_{2}}=M^{k_{2}}=8$. 


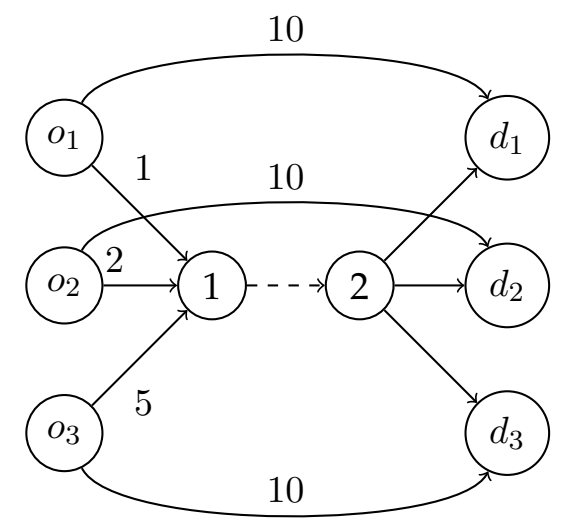

\section{Figure 4.3: Example of a network with a better bound from (HPDW-LR) with respect to (HPL-LR)}

Consider now the (HPL-LR) model, with the toll value $T=8$ (which is a possible value). Constraints (1.17e and (1.17f) for $k_{3}$ are:

$$
\begin{aligned}
p^{k_{3}} \leq M^{k_{3}} x^{k_{3}} & \Rightarrow p^{k_{3}} \leq 5 x^{k_{3}} \\
T-p^{k_{3}} \leq N\left(1-x^{k_{3}}\right) & \Rightarrow 8-p^{k_{3}} \leq 9\left(1-x^{k_{3}}\right)
\end{aligned}
$$

Therefore we have:

$$
9 x^{k_{3}}-1 \leq p^{k_{3}} \leq 5 x^{k_{3}} \Rightarrow 9 x^{k_{3}}-1 \leq 5 x^{k_{3}} \Rightarrow x^{k_{3}} \leq 0.25
$$

As we are maximising $p$ (and so also $x$ ), we take $x^{k_{3}}=0.25$ and consequently $p^{k_{3}} \leq$ $5 x^{k_{3}}=1.25$. Without the binary constraints some commodity $k$ may use a path $a$ such that $T_{a}>M_{a}^{k}$. Further, we loose the condition $p=T x$, in fact: $p^{k_{3}}=1.25 \neq T x^{k_{3}}=4$. The solution associated to $T=8$ is therefore $x^{k_{1}}=x^{k_{2}}=1, x^{k_{3}}=0.25, p^{k_{1}}=p^{k_{2}}=8$ and $p^{k_{3}}=1.25$, for a revenue of 17.25 , which is a feasible solution for (HPL-LR) and consequently provides a lower bound on the optimal value of (HPL-LR) (in fact it is the optimal solution).

Therefore for this example $F(\mathrm{HPDW}-\mathrm{LR}) \subset F(\mathrm{HPL}-\mathrm{LR})$.

In the following section we report numerical results concerning the linear relaxation optimal values of (HPL) and (HPDW) formulations on our tests instances.

More generally, the linear relaxation of the Dantzig-Wolfe reformulation always finds an optimal integer solution for the one toll path case, as it intrinsically explores all the feasible integer solutions. 
Proposition 4.5.2 (Integrality of (HPDW) for the one toll path case). If we consider a network with only one toll path, the optimal solution of (HPDW-LR) is integer.

Proof. We know that for this case $T \in\left\{M^{k}: k \in \mathcal{K}\right\} \cup\{0\}$ (see Section 1.4.2). When solving (SP) (see Section 4.3.2 we exactly explore these values for $T$ and find corresponding integer assignment variables. Solving (MP) corresponds to choose a convex combination of these values, such that at optimality the toll and the corresponding assignment providing the bigger revenue will be chosen, giving an integer solution.

Consider now the extended formulation (HPEF) we introduced in Section 4.4 its linear relaxation optimal value coincides with the linear relaxation optimal value of the Dantzig-Wolfe reformulation. Moreover, as shown in the following proposition, there exists a one to one correspondence between $F$ (HPDW-LR) and F(HPEF-LR) preserving the value of the objective function.

Proposition 4.5.3 (Correspondence between $F$ (HPDW-LR) and $F$ (HPEF-LR)). Each feasible solution of (HPDW-LR) can be transformed into a feasible solution of (HPEF-LR), and vice-versa.

Proof. A feasible solution of (HPDW-LR) is composed by a set of columns $j \in \mathcal{J}$, the corresponding parameters $\left(T_{a}^{j}, x_{a}^{k, j}, p_{a}^{k, j}\right)$ and the values of variables $\lambda_{a}^{j}$, satisfying the (MP) and the (SP) of Sections 4.3.1 and 4.3.2. A feasible solution of (HPEF-LR) is represented by the value of variables $\left(z_{a}^{l}, u_{a}^{l}, w_{a}^{k l}, v_{a}^{k l}\right)$, satisfying the formulation of Section 4.4 .

First consider a feasible solution of (HPDW-LR), we show that we can transform it into a feasible solution of (HPEF-LR). Consider each $a \in \mathcal{A}$ and the ordered set of commodities introduced in Section 4.4 (i.e. $m_{a}^{l} \leq m_{a}^{l+1}$ ), we construct the (HPEF-LR) solution as follows:

- set $z_{a}^{l}=u_{a}^{l}=\sum_{j \in \mathcal{J}}\left\{\lambda_{a}^{j}: T_{a}^{j}=m_{a}^{l+1}\right\}$; we know that $\exists l: m_{a}^{l}=T_{a}^{j}$ by the definition of $T_{a}^{j}$ parameters given in Section 4.3.2.

- set $w_{a}^{k l}=v_{a}^{k l}=\sum_{j \in \mathcal{J}}\left\{\lambda_{a}^{j}: T_{a}^{j}=m_{a}^{l+1}\right.$ and $\left.x_{a}^{k, j}=1\right\}$; we have that $w_{a}^{k l}>0$ only if $l<r_{a}(k)$, which respects the definition given by Equation (4.23);

We show that this solution is feasible for (HPEF-LR): 
constraints 4.30b): each constraint can be rewritten as follows:

$$
\begin{aligned}
& \sum_{a \in \mathcal{A}}\left[\sum_{l \in \mathcal{K}: l<r_{a}(k)} w_{a}^{k l}\left(m_{a}^{l}-M_{a}^{k}\right)+\sum_{l \in \mathcal{K}: l<r_{a}(k)} v_{a}^{k l}\left(m_{a}^{l+1}-m_{a}^{l}\right)\right]-\sum_{l \in \mathcal{K}} m_{b}^{l} z_{b}^{l}-\sum_{l \in \mathcal{K}} u_{b}^{l}\left(m_{b}^{l+1}-m_{b}^{l}\right)= \\
= & \sum_{a \in \mathcal{A}}\left[\sum_{l \in \mathcal{K}: l<r_{a}(k)} \sum_{j \in \mathcal{J}}\left\{\lambda_{a}^{j}: T_{a}^{j}=m_{a}^{l+1} \text { and } x_{a}^{k, j}=1\right\}\left(m_{a}^{l}-M_{a}^{k}\right)+\right. \\
& \left.+\sum_{l \in \mathcal{K}: l<r_{a}(k)} \sum_{j \in \mathcal{J}}\left\{\lambda_{a}^{j}: T_{a}^{j}=m_{a}^{l+1} \text { and } x_{a}^{k, j}=1\right\}\left(m_{a}^{l+1}-m_{a}^{l}\right)\right]+ \\
& -\sum_{l \in \mathcal{K}} m_{b}^{l} \sum_{j \in \mathcal{J}}\left\{\lambda_{b}^{j}: T_{b}^{j}=m_{b}^{l+1}\right\}-\sum_{l \in \mathcal{K}} \sum_{j \in \mathcal{J}}\left\{\lambda_{b}^{j}: T_{b}^{j}=m_{b}^{l+1}\right\}\left(m_{b}^{l+1}-m_{b}^{l}\right)= \\
= & \sum_{a \in \mathcal{A}} \sum_{l \in \mathcal{K}: l<r_{a}(k)} \sum_{j \in \mathcal{J}}\left\{\lambda_{a}^{j}: T_{a}^{j}=m_{a}^{l+1} \text { and } x_{a}^{k, j}=1\right\}\left(m_{a}^{l+1}-M_{a}^{k}\right)-\sum_{l \in \mathcal{K}} \sum_{j \in \mathcal{J}}\left\{\lambda_{b}^{j}: T_{b}^{j}=m_{b}^{l+1}\right\} m_{b}^{l+1}= \\
= & \sum_{a \in \mathcal{A}} \sum_{l \in \mathcal{K}: l<r_{a}(k)} \sum_{j \in \mathcal{J}} x_{a}^{k, j}\left\{\lambda_{a}^{j}: T_{a}^{j}=m_{a}^{l+1}\right\}\left(m_{a}^{l+1}-M_{a}^{k}\right)-\sum_{l \in \mathcal{K}} \sum_{j \in \mathcal{J}}\left\{\lambda_{b}^{j}: T_{b}^{j}=m_{b}^{l+1}\right\} m_{b}^{l+1}= \\
= & \sum_{a \in \mathcal{A}} \sum_{j \in \mathcal{J}} x_{a}^{k, j} \lambda_{a}^{j}\left(T_{a}^{j}-M_{a}^{k}\right)-\sum_{j \in \mathcal{J}} \lambda_{b}^{j} T_{b}^{j}=\sum_{a \in \mathcal{A}} \sum_{j \in \mathcal{J}} \lambda_{a}^{j}\left(p_{a}^{k, j}-M_{a}^{k} x_{a}^{k, j}\right)-\sum_{j \in \mathcal{J}} \lambda_{b}^{j} T_{b}^{j} \leq-M_{b}^{k}
\end{aligned}
$$

which is verified by constraints $4.10 \mathrm{~b}$ ) of (MP);

constraints 4.30c): each constraint can be rewritten as follows:

$$
\begin{aligned}
& \sum_{l \in \mathcal{K}: l<r_{a}(k)} w_{a}^{k l}\left(m_{a}^{l}-M_{a}^{k}\right)+\sum_{l \in \mathcal{K}: l<r_{a}(k)} v_{a}^{k l}\left(m_{a}^{l+1}-m_{a}^{l}\right)= \\
& =\sum_{l \in \mathcal{K}: l<r_{a}(k)} \sum_{j \in \mathcal{J}}\left\{\lambda_{a}^{j}: T_{a}^{j}=m_{a}^{l+1} \text { and } x_{a}^{k, j}=1\right\}\left(m_{a}^{l}-M_{a}^{k}\right)+ \\
& \quad+\sum_{l \in \mathcal{K}: l<r_{a}(k)} \sum_{j \in \mathcal{J}}\left\{\lambda_{a}^{j}: T_{a}^{j}=m_{a}^{l+1} \text { and } x_{a}^{k, j}=1\right\}\left(m_{a}^{l+1}-m_{a}^{l}\right)= \\
& =\sum_{l \in \mathcal{K}: l<r_{a}(k)} \sum_{j \in \mathcal{J}}\left\{\lambda_{a}^{j}: T_{a}^{j}=m_{a}^{l+1} \text { and } x_{a}^{k, j}=1\right\}\left(m_{a}^{l+1}-M_{a}^{k}\right)= \\
& \quad \ldots \text { (as for the previous one }) \ldots \\
& =\sum_{j \in \mathcal{J}} \lambda_{a}^{j}\left(p_{a}^{k, j}-M_{a}^{k} x_{a}^{k, j}\right) \leq 0
\end{aligned}
$$

as $\lambda_{a}^{j} \geq 0$ and $\left(p_{a}^{k, j}-M_{a}^{k} x_{a}^{k, j}\right) \leq 0$ for all columns by constraints $4.13 \mathrm{~b}$ of (SP);

constraints 4.30d): each constraint can be rewritten as follows:

$\sum_{a \in \mathcal{A}} \sum_{l \in \mathcal{K}: l<r_{a}(k)} w_{a}^{k l}=\sum_{a \in \mathcal{A}} \sum_{l \in \mathcal{K}: l<r_{a}(k)} \sum_{j \in \mathcal{J}}\left\{\lambda_{a}^{j}: T_{a}^{j}=m_{a}^{l+1}\right.$ and $\left.x_{a}^{k, j}=1\right\}=\sum_{a \in \mathcal{A}} \sum_{j \in \mathcal{J}} x_{a}^{k, j} \lambda_{a}^{j} \leq 1$

which is verified by constraints $4.10 \mathrm{c}$ ) of $(\mathrm{MP})$;

constraints 4.30e): each constraint can be rewritten as follows:

$$
\sum_{l \in \mathcal{K}} z_{a}^{l}=\sum_{l \in \mathcal{K}} \sum_{j \in \mathcal{J}}\left\{\lambda_{a}^{j}: T_{a}^{j}=m_{a}^{l+1}\right\}=\sum_{j \in \mathcal{J}} \lambda_{a}^{j}=1
$$


which is verified by constraints (4.10d) of (MP);

constraints 4.30f): $u_{a}^{l} \leq z_{a}^{l}$ is verified as $u_{a}^{l}=z_{a}^{l}$ by construction;

constraints 4.30g): $w_{a}^{k l} \leq z_{a}^{l}$ is verified as we have:

$$
w_{a}^{k l}=\sum_{j \in \mathcal{J}}\left\{\lambda_{a}^{j}: T_{a}^{j}=m_{a}^{l+1} \text { and } x_{a}^{k, j}=1\right\} \leq \sum_{j \in \mathcal{J}}\left\{\lambda_{a}^{j}: T_{a}^{j}=m_{a}^{l+1}\right\}=z_{a}^{l} ;
$$

constraints 4.30h): $v_{a}^{k l} \leq w_{a}^{k l}$ is verified as $v_{a}^{k l}=w_{a}^{k l}$ by construction;

constraints 4.30i): $v_{a}^{k l} \geq u_{a}^{l}+w_{a}^{k l}-z_{a}^{l}$ is verified as $v_{a}^{k l}=w_{a}^{k l}$ and $u_{a}^{l}=z_{a}^{l}$;

constraints 4.30j): $v_{a}^{k l} \leq u_{a}^{l}$ is verified similarly to constraint 4.30g);

constraints 4.30k): the relaxed version is $0 \leq z_{a}^{l} \leq 1$ and $0 \leq w_{a}^{k l} \leq 1$; variables are non negative by construction and smaller or equal to one as $w_{a}^{k l}=v_{a}^{k l}=$ $\sum_{j}\left\{\lambda_{a}^{j}: T_{a}^{j}=m_{a}^{l+1}\right.$ and $\left.x_{a}^{k, j}=1\right\} \leq \sum_{j} \lambda_{a}^{j}=1$ by constraints 4.10d of (MP);

constraints 4.301): variables $u_{a}^{l}$ and $v_{a}^{k l}$ are non negative by construction.

Consider then a feasible solution of (HPEF-LR), we show that we can transform it into a feasible solution of (HPDW-LR). To construct the (HPDW-LR) solution, we create, for each $a \in \mathcal{A}$ and $l \in \mathcal{K}$, four columns $j_{1}, j_{2}, j_{3}$ and $j_{4}$ with the following values:

column $j_{1}: \lambda_{a}^{j_{1}}=v_{a}^{k l}$;

- $T_{a}^{j_{1}}=m_{a}^{l+1}$;

- for each $k \in \mathcal{K}$ set $x_{a}^{k, j_{1}}=1$ if $w_{a}^{k l}>0,0$ otherwise;

- for each $k \in \mathcal{K}$ set $p_{a}^{k, j_{1}}=x_{a}^{k, j_{1}} T_{a}^{j_{1}}=m_{a}^{l+1}$ if $w_{a}^{k l}>0,0$ otherwise;

column $j_{2}: \lambda_{a}^{j_{2}}=w_{a}^{k l}-v_{a}^{k l}$;

- $T_{a}^{j_{2}}=m_{a}^{l}$;

- for each $k \in \mathcal{K}$ set $x_{a}^{k, j_{2}}=1$ if $w_{a}^{k l}>0,0$ otherwise;

- for each $k \in \mathcal{K}$ set $p_{a}^{k, j_{2}}=x_{a}^{k, j_{2}} T_{a}^{j_{2}}=m_{a}^{l}$ if $w_{a}^{k l}>0,0$ otherwise;

column $j_{3}: \lambda_{a}^{j_{3}}=u_{a}^{l}-v_{a}^{k l}$;

- $T_{a}^{j_{3}}=m_{a}^{l+1}$;

- for each $k \in \mathcal{K}$ set $x_{a}^{k, j_{3}}=0$;

- for each $k \in \mathcal{K}$ set $p_{a}^{k, j_{3}}=0$;

column $j_{4}: \lambda_{a}^{j_{4}}=\left(z_{a}^{l}-u_{a}^{l}\right)-\left(w_{a}^{k l}-v_{a}^{k l}\right)$;

- $T_{a}^{j_{4}}=m_{a}^{l}$;

- for each $k \in \mathcal{K}$ set $x_{a}^{k, j_{4}}=0$; 
- for each $k \in \mathcal{K}$ set $p_{a}^{k, j_{4}}=0$.

We show that this solution is feasible for (HPDW-LR):

constraints 4.10b) of (MP): each constraint can be rewritten as follows:

$$
\begin{aligned}
& \sum_{a \in \mathcal{A}} \sum_{j \in \mathcal{J}} \lambda_{a}^{j}\left(p_{a}^{k, j}-M_{a}^{k} x_{a}^{k, j}\right)-\sum_{j \in \mathcal{J}} \lambda_{b}^{j} T_{b}^{j}=\sum_{a \in \mathcal{A}} \sum_{j \in \mathcal{J}} \lambda_{a}^{j}\left(T_{a}^{j} x_{a}^{k, j}-M_{a}^{k} x_{a}^{k, j}\right)-\sum_{j \in \mathcal{J}} \lambda_{b}^{j} T_{b}^{j}= \\
= & \sum_{a \in \mathcal{A}} \sum_{j \in \mathcal{J}}\left(T_{a}^{j}-M_{a}^{k}\right)\left\{\lambda_{a}^{j}: x_{a}^{k, j}=1\right\}-\sum_{j \in \mathcal{J}} \lambda_{b}^{j} T_{b}^{j}= \\
= & \sum_{a \in \mathcal{A}} \sum_{l \in \mathcal{K}: l<r_{a}(k)}\left[\left(m_{a}^{l+1}-M_{a}^{k}\right) v_{a}^{k l}+\left(m_{a}^{l}-M_{a}^{k}\right)\left(w_{a}^{k l}-v_{a}^{k l}\right)\right]+ \\
& \quad-\sum_{l \in \mathcal{K}}\left[M_{b}^{l+1}\left(v_{b}^{k l}+u_{b}^{l}-v_{b}^{k l}\right)+M_{b}^{l}\left(w_{a}^{k l}-v_{a}^{k l}+z_{a}^{l}-u_{a}^{l}-w_{a}^{k l}+v_{a}^{k l}\right)\right]= \\
= & \sum_{a \in \mathcal{A}} \sum_{l \in \mathcal{K}: l<r_{a}(k)}\left[\left(m_{a}^{l}-M_{a}^{k}\right) w_{a}^{k l}+\left(m_{a}^{l+1}-m_{a}^{l}\right) v_{a}^{k l}\right]-\sum_{l \in \mathcal{K}}\left[\left(m_{a}^{l+1}-m_{a}^{l}\right) u_{a}^{l}+M_{b}^{l} z_{b}^{l}\right] \leq-M_{b}^{k}
\end{aligned}
$$

which is valid by constraints $4.30 \mathrm{~b}$ ) of (HPEF-LR);

constraints $4.10 \mathrm{c}$ ) of (MP): each constraint can be rewritten as follows:

$$
\sum_{a \in \mathcal{A}} \sum_{j \in \mathcal{J}} \lambda_{a}^{j} x_{a}^{k, j}=\sum_{a \in \mathcal{A}} \sum_{j \in \mathcal{J}}\left\{\lambda_{a}^{j}: x_{a}^{k, j}=1\right\}=\sum_{a \in \mathcal{A}} \sum_{l \in \mathcal{K}: l<r_{a}(k)}\left(v_{a}^{k l}+w_{a}^{k l}-v_{a}^{k l}\right)=\sum_{a \in \mathcal{A}} \sum_{l \in \mathcal{K}: l<r_{a}(k)} w_{a}^{k l} \leq 1
$$

which is verified by constraints 4.30d;

constraints 4.10d) of (MP): each constraint can be rewritten as follows:

$$
\sum_{j \in \mathcal{J}} \lambda_{a}^{j}=\sum_{l \in \mathcal{K}}\left(v_{a}^{k l}+w_{a}^{k l}-v_{a}^{k l}+u_{a}^{l}-v_{a}^{k l}+z_{a}^{l}-u_{a}^{l}-w_{a}^{k l}+v_{a}^{k l}\right)=\sum_{l \in \mathcal{K}} z_{a}^{l}=1
$$

which is verified by constraints 4.30e;

constraints 4.10e of (MP): the relaxed version is $0 \leq \sum_{j} \lambda_{a}^{j} x_{a}^{k, j} \leq 1$, and we have:

$$
\sum_{j \in \mathcal{J}} \lambda_{a}^{j} x_{a}^{k, j}=\sum_{j \in \mathcal{J}}\left\{\lambda_{a}^{j}: x_{a}^{k, j}=1\right\}=\sum_{l \in \mathcal{K}: l<r_{a}(k)}\left(v_{a}^{k l}+w_{a}^{k l}-v_{a}^{k l}\right)=\sum_{l \in \mathcal{K}: l<r_{a}(k)} w_{a}^{k l}
$$

which is non negative as $w_{a}^{k l} \geq 0$ by the linear relaxation of constraints 4.30k and smaller or equal than one by constraints (4.30d);

constraints 4.10f of (MP): $\lambda_{a}^{j} \geq 0$ is verified for each column as:

$j_{1}: \quad \lambda_{a}^{j_{1}}=v_{a}^{k l} \geq 0$ by constraints 4.301;

$j_{2}: \quad \lambda_{a}^{j_{2}}=w_{a}^{k l}-v_{a}^{k l} \geq 0$ as $v_{a}^{k l} \leq w_{a}^{k l}$ by constraints 4.30h;

$j_{3}: \quad \lambda_{a}^{j_{3}}=u_{a}^{l}-v_{a}^{k l} \geq 0$ as $v_{a}^{k l} \leq u_{a}^{k l}$ by constraints 4.30j; ;

$j_{4}: \quad \lambda_{a}^{j_{4}}=z_{a}^{l}-u_{a}^{l}-w_{a}^{k l}+v_{a}^{k l} \geq 0$ as $v_{a}^{k l} \geq u_{a}^{l}+w_{a}^{k l}-z_{a}^{l}$ by constraints 4.30i. 
constraints $4.13 \mathrm{~b}$ of (SP): the constraint, for each column $j$, can be rewritten as:

$$
\begin{aligned}
& p_{a}^{k, j}-M_{a}^{k} x_{a}^{k, j}=x_{a}^{k, j} T_{a}^{j}-M_{a}^{k} x_{a}^{k, j}=x_{a}^{k, j}\left(T_{a}^{j}-M_{a}^{k}\right)= \\
= & \left\{\begin{array}{l}
0 \quad \text { if } x_{a}^{k, j}=0 \\
T_{a}^{j}-M_{a}^{k} \leq 0
\end{array} \text { if } x_{a}^{k, j}=1 \Rightarrow w_{a}^{k l}>0 \Rightarrow l<k\right.
\end{aligned}
$$

which is valid as $T_{a}^{j}=m_{a}^{l+1} \leq M_{a}^{k}$ for $j=j_{1}$ and $T_{a}^{j}=m_{a}^{l} \leq M_{a}^{k}$ for $j=j_{2}$;

constraints $4.13 \mathrm{c}$ of (SP): $p_{a}^{k, j}=x_{a}^{k, j} T_{a}^{j}$ is verified by construction;

constraints (4.13d) of (SP): $x_{a}^{k, j} \in\{0,1\}$ is verified by construction;

constraints $4.13 \mathrm{e}$ of (SP): $T_{a}^{j} \geq 0$ is verified by construction.

Corollary 4.5.4. The optimal solution values of (HPDW-LR) and (HPEF-LR) coincide.

Proof. In Proposition 4.5.3 we showed that any feasible solution of (HPDW-LR) can be transformed into a feasible solution of (HPEF-LR), and viceversa. We now show that the proposed transformations preserve the objective function value.

First we show that objective function value of the feasible solution of (HPEF-LR) obtained starting from a feasible solution of (HPDW-LR) is equivalent to the objective function value of this (HPDW-LR) solution:

$$
\begin{aligned}
& \sum_{a \in \mathcal{A}} \sum_{k \in \mathcal{K}} \eta^{k}\left[\sum_{l \in \mathcal{K}: l<r_{a}(k)} m_{a}^{l} w_{a}^{k l}+\sum_{l \in \mathcal{K}: l<r_{a}(k)} v_{a}^{k l}\left(m_{a}^{l+1}-m_{a}^{l}\right)\right]= \\
= & \sum_{a \in \mathcal{A}} \sum_{k \in \mathcal{K}} \eta^{k}\left[\sum_{l \in \mathcal{K}: l<r_{a}(k)} m_{a}^{l} \sum_{j \in \mathcal{J}}\left\{\lambda_{a}^{j}: T_{a}^{j}=m_{a}^{l+1} \text { and } x_{a}^{k, j}=1\right\}+\right. \\
& \left.+\sum_{l \in \mathcal{K}: l<r_{a}(k)} \sum_{j \in \mathcal{J}}\left\{\lambda_{a}^{j}: T_{a}^{j}=m_{a}^{l+1} \text { and } x_{a}^{k, j}=1\right\}\left(m_{a}^{l+1}-m_{a}^{l}\right)\right]= \\
= & \sum_{a \in \mathcal{A}} \sum_{k \in \mathcal{K}} \eta^{k} \sum_{l \in \mathcal{K}: l<r_{a}(k)} m_{a}^{l+1} \sum_{j \in \mathcal{J}}\left\{\lambda_{a}^{j}: T_{a}^{j}=m_{a}^{l+1} \text { and } x_{a}^{k, j}=1\right\}= \\
= & \sum_{a \in \mathcal{A}} \sum_{k \in \mathcal{K}} \eta^{k} \sum_{j \in \mathcal{J}} \lambda_{a}^{j} x_{a}^{k, j} T_{a}^{j}=\sum_{a \in \mathcal{A}} \sum_{k \in \mathcal{K}} \sum_{j \in \mathcal{J}} \lambda_{a}^{j} \eta^{k} p_{a}^{k, j}
\end{aligned}
$$

We then show that objective function value of the feasible solution of (HPDW-LR) obtained starting from a feasible solution of (HPEF-LR) is equivalent to the objective function value of this (HPEF-LR) solution:

$$
\begin{aligned}
& \sum_{a \in \mathcal{A}} \sum_{k \in \mathcal{K}} \sum_{j \in \mathcal{J}} \lambda_{a}^{j} \eta^{k} p_{a}^{k, j}=\sum_{a \in \mathcal{A}} \sum_{k \in \mathcal{K}} \sum_{j \in \mathcal{J}} \lambda_{a}^{j} \eta^{k} x_{a}^{k, j} T_{a}^{j}=\sum_{a \in \mathcal{A}} \sum_{k \in \mathcal{K}} \eta^{k} \sum_{j \in \mathcal{J}} T_{a}^{j}\left\{\lambda_{a}^{j}: x_{a}^{k, j}=1\right\}= \\
= & \sum_{a \in \mathcal{A}} \sum_{k \in \mathcal{K}} \eta^{k} \sum_{l \in \mathcal{K}: l<r_{a}(k)}\left[m_{a}^{l+1} v_{a}^{k l}+m_{a}^{l}\left(w_{a}^{k l}-v_{a}^{k l}\right)\right]=\sum_{a \in \mathcal{A}} \sum_{k \in \mathcal{K}} \eta^{k} \sum_{l \in \mathcal{K}: l<r_{a}(k)}\left[m_{a}^{l} w_{a}^{k l}+\left(m_{a}^{l+1}-m_{a}^{l}\right) v_{a}^{k l}\right] .
\end{aligned}
$$


As we can transform any feasible solution of (HPDW-LR) (resp. (HPEF-LR)) into a feasible solution of (HPEF-LR) (resp. (HPDW-LR)) with the same objective function value, we can do it for the optimal solution of (HPDW-LR) (resp. (HPEF-LR)). Then, the optimal solution value of (HPDW-LR) (resp. (HPEF-LR)) is a lower bound for the optimal solution value of (HPEF-LR) (resp. (HPDW-LR)). However, if there is another feasible solution of (HPEF-LR) (resp. (HPDW-LR)) with an higher objective function value, we can transform it into another feasible solution of (HPDW-LR) (resp. (HPEFLR)) with the same objective function value, which is higher than the optimal one, yielding to a contradiction. Therefore, the optimal solution values of (HPDW-LR) and (HPEF-LR) coincide.

\subsubsection{Numerical results}

In Table 4.2 we report numerical results concerning the linear relaxation of (HPL), (HPDW) and (HPEF) formulations. We report the gap between the optimal integer value and the linear relaxation optimal value and the time to solve the linear relaxation in seconds. We do not report the gap of (HPEF-LR) as it is the same as the one of (HPDW-LR). Instances are described in Section 4.3.4.

We were not able to find the optimal integer solution for some of the bigger instances (solving HPL): in this case we report the gap from the best integer solution found in 5 hours of computational time ${ }^{*}$ represents the number of instances not solved to optimality). Note that all Shioda et al. and A1 instances have been solved to optimality in the time limit of 5 hours, whilst it is not the case for the complete and partial graph instances with 56 and 90 commodities.

We notice that instances on partial graph show to have a very large gap (up to $50 \%$ for (HPL)), and Shioda et al.'s ones a small value (up to $2.5 \%$ for (HPL)). A1 instances also have a relatively small gap, up to around $6 \%$ for (HPL).

As expected from theoretical results, the gap of (HPDW-LR) and (HPEF-LR) are always smaller than the gap of (HPL-LR), with a greater difference between them when the number of toll paths is smaller or the number of commodities is bigger. In particular, for instances with 90 or 80 commodities and 20 toll paths, (HPDW-LR) and (HPEF-LR) are able to halve the gap.

For all classes of instances except the A1, (HPL-LR) has a smaller solution time than (HPDW-LR), with a large difference for big instances. However, for A1 instances (HPDW-LR) formulation is competitive with respect to (HPL-LR), and for instances with 21 and 55 commodities it even outperforms it.

In Table 4.3 we report the ratio of the gap and the solution time of (HPL-LR) formulation divided by the same value of (HPDW-LR) formulation: if the ratio is greater than one it means that (HPDW) is performing better, if it is less than one (HPL) is better.

For the gap, we notice that for all class of instances the improvements of (HPDW) formulation increase when the number of commodity increases, whilst they decrease 


\begin{tabular}{|c|c|c|c|c|c|c|c|c|c|c|}
\hline \multirow{2}{*}{\multicolumn{2}{|c|}{$\begin{array}{l}\text { Complete } \\
\text { graph }\end{array}$}} & \multicolumn{3}{|c|}{20 commodities } & \multicolumn{3}{|c|}{56 commodities } & \multicolumn{3}{|c|}{90 commodities } \\
\hline & & $20 \mathrm{a}$ & $56 \mathrm{a}$ & $90 \mathrm{a}$ & $20 a$ & $56 a^{* 7}$ & $90 a^{* 10}$ & $20 \mathrm{a}$ & $56 a^{* 10}$ & $90 a^{* 10}$ \\
\hline \multirow[t]{2}{*}{$(-\mathrm{L})$} & Gap & $6.19 \%$ & $3.71 \%$ & $3.15 \%$ & $12.82 \%$ & $11.18 \%$ & $11.51 \%$ & $15.33 \%$ & $16.88 \%$ & $17.47 \%$ \\
\hline & Time & 0.048 & 0.282 & 0.767 & 0.184 & 1.514 & 4.592 & 0.448 & 4.849 & 6.084 \\
\hline \multirow[t]{2}{*}{$(-\mathrm{DW})$} & Gap & $4.75 \%$ & $3.52 \%$ & $2.98 \%$ & $7.72 \%$ & $9.57 \%$ & $10.68 \%$ & $7.94 \%$ & $13.94 \%$ & $15.98 \%$ \\
\hline & Time & 0.199 & 0.726 & 1.57 & 4.13 & 12.7 & 22.3 & 25.84 & 62.26 & 108.99 \\
\hline$(-E F)$ & Time & 1.21 & 6.84 & 18.74 & 30.76 & 139.69 & 280.99 & 185.13 & 833.40 & 1289.16 \\
\hline \multirow{2}{*}{\multicolumn{2}{|c|}{$\begin{array}{l}\text { Partial } \\
\text { graph }\end{array}$}} & \multicolumn{3}{|c|}{20 commodities } & \multicolumn{3}{|c|}{56 commodities } & \multicolumn{3}{|c|}{90 commodities } \\
\hline & & $20 \mathrm{a}$ & $56 \mathrm{a}$ & $90 \mathrm{a}$ & $20 \mathrm{a}$ & $56 a^{* 10}$ & $90 a^{* 10}$ & $20 a^{* 9}$ & $56 a^{* 10}$ & $90 \mathrm{a}^{* 10}$ \\
\hline \multirow[t]{2}{*}{$(-\mathrm{L})$} & Gap & $22.78 \%$ & $20.49 \%$ & $19.58 \%$ & $36.37 \%$ & $41.91 \%$ & $40.69 \%$ & $42.87 \%$ & $48.67 \%$ & $52.06 \%$ \\
\hline & Time & 0.042 & 0.28 & 0.858 & 0.207 & 1.871 & 6.244 & 0.519 & 3.767 & 10.6 \\
\hline \multirow[t]{2}{*}{$(-\mathrm{DW})$} & Gap & $17.64 \%$ & $19.30 \%$ & $19.03 \%$ & $22.19 \%$ & $36.33 \%$ & $37.67 \%$ & $23.95 \%$ & $39.84 \%$ & $46.52 \%$ \\
\hline & Time & 0.171 & 0.687 & 1.443 & 3.026 & 10.99 & 21.23 & 18.54 & 55.71 & 102.87 \\
\hline$(-E F)$ & Time & 1.30 & 6.50 & 17.52 & 24.15 & 139.41 & 261.51 & 137.56 & 822.65 & 1335.32 \\
\hline \multirow{2}{*}{\multicolumn{2}{|c|}{$\begin{array}{l}\text { Shioda et al. } \\
\text { instances }\end{array}$}} & \multicolumn{3}{|c|}{40 commodities } & \multicolumn{3}{|c|}{60 commodities } & \multicolumn{3}{|c|}{80 commodities } \\
\hline & & $20 \mathrm{a}$ & $40 \mathrm{a}$ & $60 \mathrm{a}$ & $20 \mathrm{a}$ & $40 \mathrm{a}$ & $60 \mathrm{a}$ & $20 \mathrm{a}$ & $40 \mathrm{a}$ & $60 \mathrm{a}$ \\
\hline \multirow[t]{2}{*}{$(-\mathrm{L})$} & Gap & $1.69 \%$ & $0.28 \%$ & $0.07 \%$ & $2.39 \%$ & $0.58 \%$ & $0.27 \%$ & $2.42 \%$ & $0.68 \%$ & $0.25 \%$ \\
\hline & Time & 0.09 & 0.21 & 0.48 & 0.13 & 0.39 & 0.78 & 0.18 & 0.55 & 1.16 \\
\hline \multirow[t]{2}{*}{$(-\mathrm{DW})$} & Gap & $1.00 \%$ & $0.20 \%$ & $0.05 \%$ & $1.24 \%$ & $0.37 \%$ & $0.20 \%$ & $1.09 \%$ & $0.36 \%$ & $0.15 \%$ \\
\hline & Time & 5.51 & 7.79 & 11.39 & 43.12 & 47.04 & 56.83 & 198.38 & 221.28 & 260.09 \\
\hline$(-E F)$ & Time & 11.94 & 35.52 & 63.16 & 71.32 & 184.75 & 290.23 & 220.57 & 534.63 & 1097.96 \\
\hline \multirow{2}{*}{\multicolumn{2}{|c|}{$\begin{array}{l}\text { A1 } \\
\text { instances }\end{array}$}} & \multicolumn{3}{|c|}{21 commodities } & \multicolumn{3}{|c|}{55 commodities } & \multicolumn{3}{|c|}{91 commodities } \\
\hline & & $21 \mathrm{a}$ & $55 \mathrm{a}$ & $91 \mathrm{a}$ & $21 \mathrm{a}$ & $55 \mathrm{a}$ & $91 \mathrm{a}$ & $21 \mathrm{a}$ & $55 \mathrm{a}$ & $91 \mathrm{a}$ \\
\hline \multirow[t]{2}{*}{$(-\mathrm{L})$} & Gap & $5.30 \%$ & $2.78 \%$ & $1.41 \%$ & $6.36 \%$ & $3.57 \%$ & $1.74 \%$ & $6.26 \%$ & $4.34 \%$ & $3.12 \%$ \\
\hline & Time & 0.13 & 0.48 & 1.21 & 0.50 & 2.36 & 5.70 & 1.34 & 5.83 & 14.96 \\
\hline \multirow[t]{2}{*}{$(-\mathrm{DW})$} & Gap & $3.80 \%$ & $2.30 \%$ & $1.35 \%$ & $3.14 \%$ & $2.52 \%$ & $1.25 \%$ & $2.41 \%$ & $2.97 \%$ & $2.18 \%$ \\
\hline & Time & 0.07 & 0.22 & 0.46 & 0.48 & 1.69 & 3.85 & 3.14 & 8.63 & 16.45 \\
\hline$(-E F)$ & Time & 0.45 & 1.66 & 3.23 & 3.19 & 11.20 & 28.34 & 13.89 & 52.02 & 108.16 \\
\hline
\end{tabular}

Table 4.2: Numerical results on linear relaxation for (HPL), (HPDW) and (HPEF) formulations

when the number of toll path increases. Moreover, (HPDW) improvements are higher for Shioda et al. and A1 instances. As already revealed by absolute results of Table 4.2. time-wise (HPDW) formulation performs quite badly with respect to (HPL) for the first three classes of instances. For A1 instances we already noticed comparable performances. Ratios from Table 4.3 allows us to add that the time performance of (HPDW) increase when the number of toll paths increases, whilst they decrease when the number of commodities increases.

In Figure 4.4 we report the performance profile graphs for the gap between the optimal integer value and the linear relaxation optimal value, for (HPL) and (HPDW) formulations. The ordinate's axis represents the number of instances for which the gap is smaller than the corresponding $x$-axis' value, such that a higher curve means a better formulation in terms of the gap of the linear relaxation. Also from these graphs we notice a great difference regarding the gap for the two formulations.

We compare now (HPDW) and (HPEF) formulations: from results of Table 4.2 we 


\begin{tabular}{|c|c|c|c|c|c|c|c|c|c|c|}
\hline \multirow{2}{*}{$\begin{array}{l}\text { Complete } \\
\text { graph }\end{array}$} & & \multicolumn{3}{|c|}{20 commodities } & \multicolumn{3}{|c|}{56 commodities } & \multicolumn{3}{|c|}{90 commodities } \\
\hline & & $20 \mathrm{a}$ & $56 \mathrm{a}$ & $90 \mathrm{a}$ & $20 \mathrm{a}$ & $56 a$ & $90 \mathrm{a}$ & $20 \mathrm{a}$ & $56 \mathrm{a}$ & $90 \mathrm{a}$ \\
\hline \multirow[t]{2}{*}{$(-\mathrm{L}) /(-\mathrm{DW})$} & Gap & 1.3 & 1.1 & 1.1 & 1.7 & 1.2 & 1.1 & 1.9 & 1.2 & 1.1 \\
\hline & Time & 0.2 & 0.4 & 0.5 & 0.04 & 0.1 & 0.2 & 0.02 & 0.1 & 0.1 \\
\hline \multirow{2}{*}{\multicolumn{2}{|c|}{$\begin{array}{l}\text { Partial } \\
\text { graph }\end{array}$}} & \multicolumn{3}{|c|}{20 commodities } & \multicolumn{3}{|c|}{56 commodities } & \multicolumn{3}{|c|}{90 commodities } \\
\hline & & $20 \mathrm{a}$ & $56 \mathrm{a}$ & $90 \mathrm{a}$ & $20 \mathrm{a}$ & $56 a$ & $90 \mathrm{a}$ & $20 \mathrm{a}$ & $56 \mathrm{a}$ & $90 \mathrm{a}$ \\
\hline \multirow[t]{2}{*}{$(-\mathrm{L}) /(-\mathrm{DW})$} & Gap & 1.3 & 1.1 & 1 & 1.6 & 1.2 & 1.1 & 1.8 & 1.2 & 1.1 \\
\hline & Time & 0.2 & 0.4 & 0.6 & 0.1 & 0.2 & 0.3 & 0.03 & 0.1 & 0.1 \\
\hline \multirow{2}{*}{\multicolumn{2}{|c|}{$\begin{array}{l}\text { Shioda et al. } \\
\text { instances }\end{array}$}} & \multicolumn{3}{|c|}{40 commodities } & \multicolumn{3}{|c|}{60 commodities } & \multicolumn{3}{|c|}{80 commodities } \\
\hline & & $20 \mathrm{a}$ & $40 \mathrm{a}$ & $60 \mathrm{a}$ & $20 \mathrm{a}$ & $40 \mathrm{a}$ & $60 \mathrm{a}$ & $20 \mathrm{a}$ & $40 \mathrm{a}$ & $60 \mathrm{a}$ \\
\hline \multirow[t]{2}{*}{$(-\mathrm{L}) /(-\mathrm{DW})$} & Gap & 1.7 & 1.4 & 1.4 & 1.9 & 1.6 & 1.4 & 2.2 & 1.9 & 1.7 \\
\hline & Time & 0.02 & 0.03 & 0.04 & 0.01 & 0.01 & 0.01 & 0.001 & 0.002 & 0.0004 \\
\hline \multirow{2}{*}{\multicolumn{2}{|c|}{$\begin{array}{l}\text { A1 } \\
\text { instances }\end{array}$}} & \multicolumn{3}{|c|}{21 commodities } & \multicolumn{3}{|c|}{55 commodities } & \multicolumn{3}{|c|}{91 commodities } \\
\hline & & $21 \mathrm{a}$ & $55 \mathrm{a}$ & $91 \mathrm{a}$ & $21 \mathrm{a}$ & $55 \mathrm{a}$ & $91 \mathrm{a}$ & $21 \mathrm{a}$ & $55 \mathrm{a}$ & $91 \mathrm{a}$ \\
\hline \multirow[t]{2}{*}{$(-\mathrm{L}) /(-\mathrm{DW})$} & Gap & 1.4 & 1.2 & 1.0 & 2.0 & 1.4 & 1.4 & 2.6 & 1.5 & 1.4 \\
\hline & Time & 1.9 & 2.2 & 2.6 & 1.0 & 1.4 & 1.5 & 0.4 & 0.7 & 0.9 \\
\hline
\end{tabular}

Table 4.3: Performance ratio of (HPDW-LR) formulation with respect to (HPL-LR) formulation

can see that the solution time of (HPDW-LR) is significantly smaller than for (HPEFLR). The (HPEF-LR) formulation has very high solution time, up to 20 minutes for the bigger instances using up to 12GB of memory, and we are only solving the linear relaxation. For this reason this extended formulation, despite providing the same gap as the Dantzig-Wolfe reformulation and being compact and easy to implement, is not very promising to solve the integer problem. In Figure 4.5 we report the performance profile graphs for the linear relaxation of both formulations, where the $y$-axis represents the number of instances solved within the corresponding time (in seconds) in the $\mathrm{x}$ axis. These graphs also shows the big difference in performances for the solution time of (HPDW) and (HPEF) formulations, with (HPEF) being ten time slower than (HPDW) for complete and partial graph and A1 instances.

\subsection{Initialisation of the (MP), infeasibility and Farkas pricing}

The first step of a column generation algorithm is the initialisation of the (MP) with a subset of columns. Either we are able to calculate a set of columns that provide an initial feasible solution for the (MP), for instance with an heuristic, or we can start with an unfeasible (MP) and construct feasible columns within the solution procedure. Furthermore, the (MP) may become unfeasible after branching, as it will be discussed in Chapter 5, so we need to be able to make the (MP) feasible again (if possible).

The traditional way to find a first feasible solution is with a "phase I" approach, introducing artificial variables with high penalty cost. Another tool is provided by column generation, using the so-called Farkas pricing problem.

We first describe how to find initial feasible solutions with heuristics and then present 


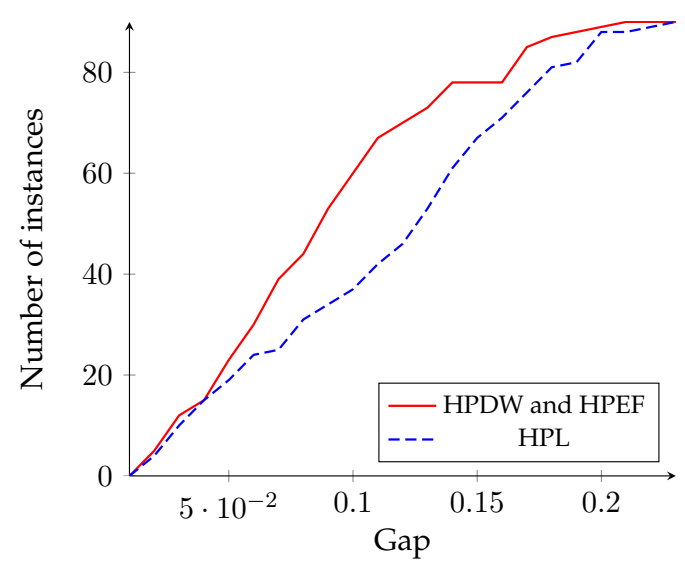

(a) Complete graph instances

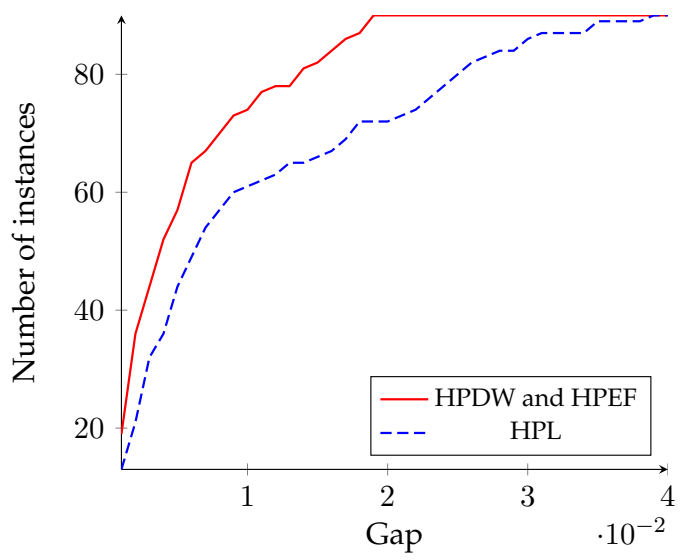

(c) Shioda et al.'s instances

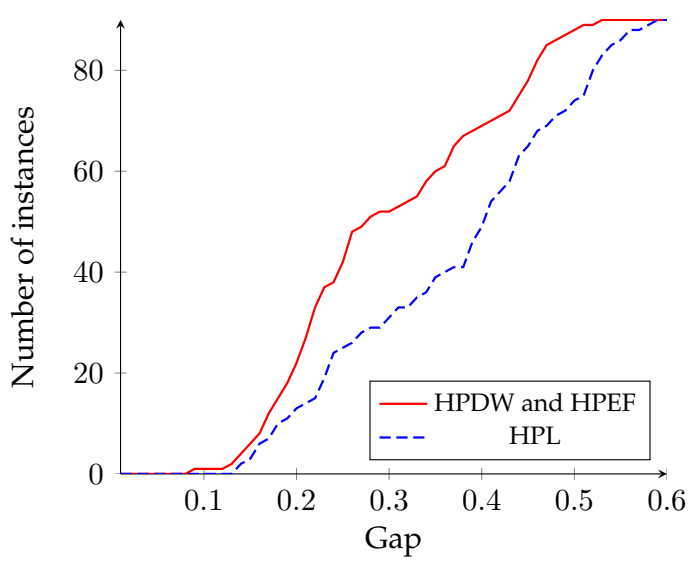

(b) Partial graph instances

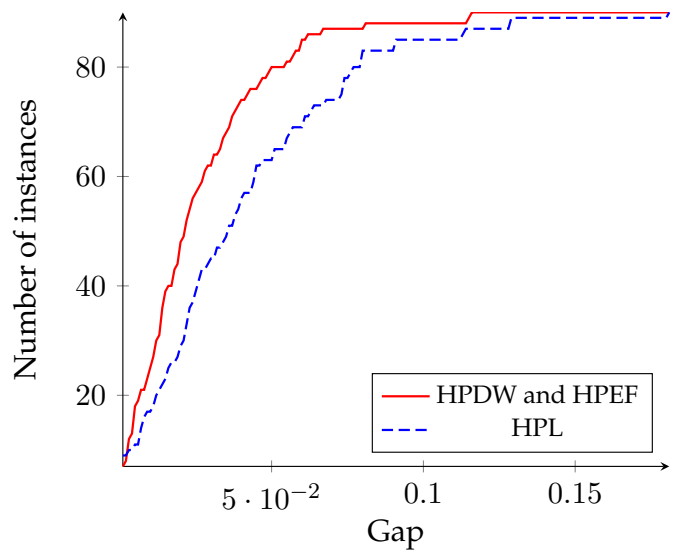

(d) A1 instances

Figure 4.4: Performance profile graphs on the gap for the linear relaxation for (HPL), (HPDW) and (HPEF)

the Farkas pricing method. We choose this technique as it is straightforward to implement, being a simple variation of the standard pricing problem.

\subsubsection{Initialisation}

A good initial set of columns may help the convergence of the column generation algorithm, even though it has been shown that sometimes excellent initial integer solutions may be detrimental to solve a linear program with column generation. A discussion on this topic with references can be found in Lübbecke and Desrosiers (2005). For our highway problem it is quite straightforward to find an initial set of feasible columns for the (MP) and we will try few different alternatives.

First of all we need one column per toll path, otherwise convexity constraints (4.10d) are not satisfied. Moreover, feasibility for the linear relaxation of the restricted (MP) all along the solution procedure with column generation is guaranteed if we have one column $j$ for each path $a$ where the coefficients $x_{a}^{k, j}$ are zero for each $k \in \mathcal{K}$ (meaning 


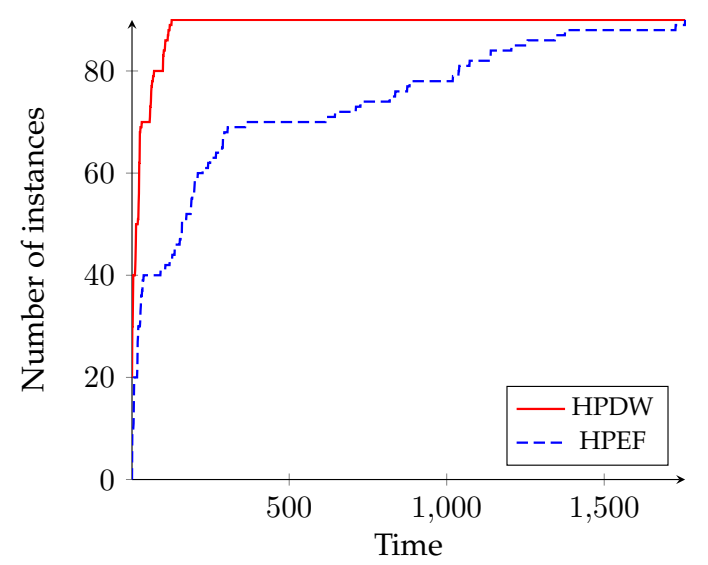

(a) Complete graph instances

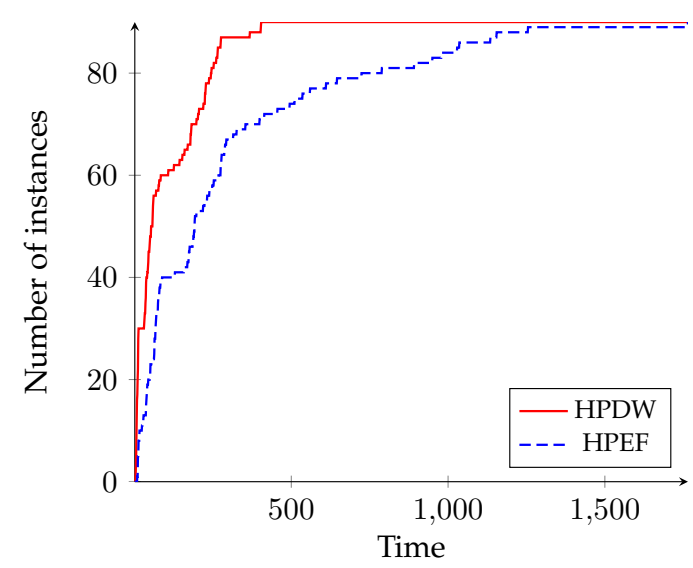

(c) Shioda et al.'s instances

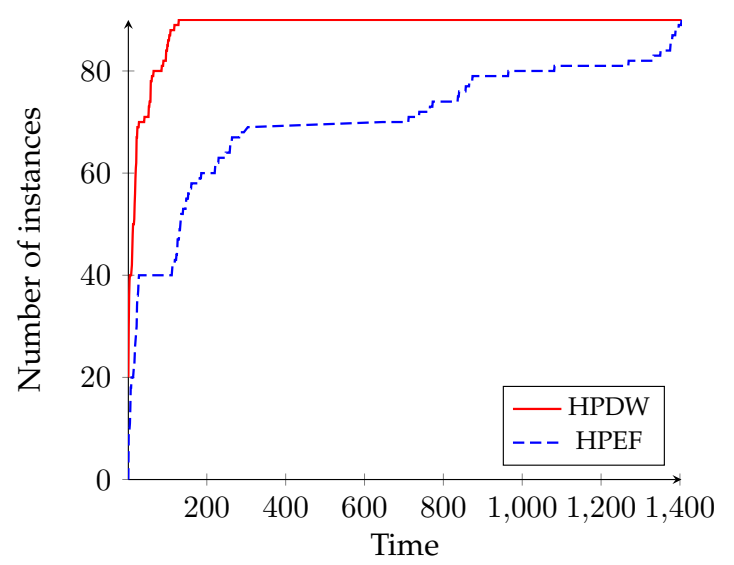

(b) Partial graph instances

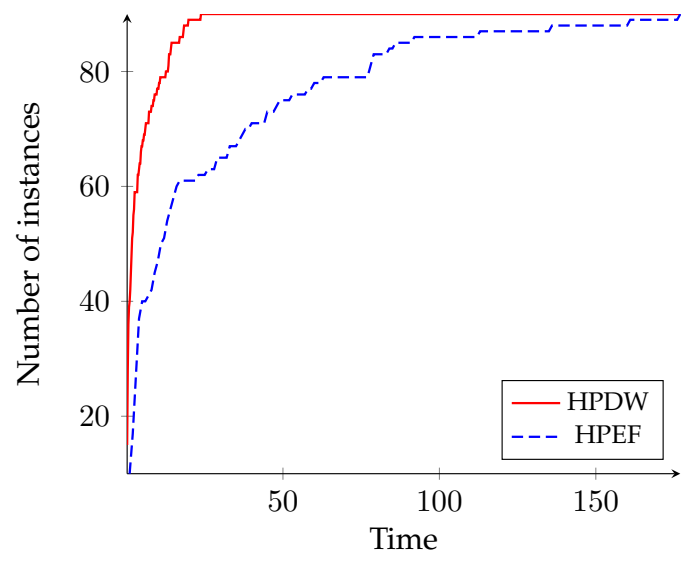

(d) A1 instances

Figure 4.5: Performance profile graphs on the solution time for the linear relaxation of (HPDW) and (HPEF)

that all commodities are assigned to their toll free path). Therefore we initialise our problem with one column $j$ for each toll path $a \in \mathcal{A}$, with the toll equal to a big value (for instance $N_{a}$ are valid values) and the corresponding $x_{a}^{k, j}=0$, for each $k \in \mathcal{K}$. The $p$ vector is equal to $T x$, such that it is null. With this set of columns we can immediately find a feasible (integer) solution for (MP), whose value is zero. This value provides us also with a valid primal (lower) bound for the integer problem, even if it is a very bad bound.

As we would like to have a better initial (integer) solution, we could try to initialise the problem with more columns. Starting the column generation algorithm with more columns may help the convergence of the algorithm.

An intuitive heuristic to find an additional set of initial columns is the following one. We generate a column $j$ for each path $a \in \mathcal{A}$ and we fix the toll $T_{a}^{j}$ equal to the smallest $M_{a}^{k}$ which is not zero. We then calculate the corresponding optimal assignment for commodities to paths ( $x_{a}^{k, j}$ values) with these tolls, solving a shortest path problem for each commodity. The $p$ vector is equal to $T x$. This heuristic provides us with a feasible 
integer solution whose value is positive in most cases.

Numerical results of Table 4.4 show the relevance of this additional initialisation when solving the linear relaxation of (HPDW), whilst results for the integer problem are reported in Chapter 5. We report the solution time in seconds and the number of generated columns, for both the initialisation with large toll values and null assignment (INIT 0) and the additional initialisation with the simple heuristic (INIT 1). For complete and partial graph instances the two initialisation strategies lead to similar results, which are slightly improved using the second initialisation strategy. Also for A1 instances the improvement from the second initialisation strategy is not significant. For Shioda et al. instances we notice that the second initialisation strategy provides a significant improvement in both the solution time and the number of generated columns, for all instance sizes.

\begin{tabular}{|c|c|c|c|c|c|c|c|c|c|c|}
\hline \multirow{2}{*}{\multicolumn{2}{|c|}{$\begin{array}{l}\text { Complete } \\
\text { graph }\end{array}$}} & \multicolumn{3}{|c|}{20 commodities } & \multicolumn{3}{|c|}{56 commodities } & \multicolumn{3}{|c|}{90 commodities } \\
\hline & & $20 \mathrm{a}$ & $56 \mathrm{a}$ & $90 \mathrm{a}$ & $20 \mathrm{a}$ & $56 \mathrm{a}$ & $90 \mathrm{a}$ & $20 \mathrm{a}$ & $56 \mathrm{a}$ & $90 \mathrm{a}$ \\
\hline \multirow[t]{2}{*}{ INIT 0} & Time & 0.199 & 0.726 & 1.57 & 4.13 & 12.7 & 22.3 & 25.84 & 62.26 & 108.99 \\
\hline & Cols & 335 & 655 & 924 & 1141 & 1765 & 2227 & 2103 & 3034 & 3749 \\
\hline \multirow[t]{2}{*}{ INIT 1} & Time & 0.116 & 0.509 & 1.14 & 3.89 & 11.84 & 21.87 & 25.71 & 61.22 & 108.73 \\
\hline & Cols & 337 & 607 & 875 & 1135 & 1741 & 2200 & 2134 & 3031 & 3767 \\
\hline \multirow{2}{*}{\multicolumn{2}{|c|}{$\begin{array}{l}\text { Partial } \\
\text { graph }\end{array}$}} & \multicolumn{3}{|c|}{20 commodities } & \multicolumn{3}{|c|}{56 commodities } & \multicolumn{3}{|c|}{90 commodities } \\
\hline & & $20 \mathrm{a}$ & $56 \mathrm{a}$ & 90 a & $20 \mathrm{a}$ & $56 \mathrm{a}$ & $90 \mathrm{a}$ & $20 \mathrm{a}$ & $56 a$ & $90 \mathrm{a}$ \\
\hline \multirow[t]{2}{*}{ INIT 0} & Time & 0.171 & 0.687 & 1.443 & 3.026 & 10.99 & 21.23 & 18.54 & 55.71 & 102.87 \\
\hline & Cols & 301 & 625 & 891 & 974 & 1699 & 2199 & 1853 & 2926 & 3747 \\
\hline \multirow[t]{2}{*}{ INIT 1} & Time & 0.159 & 0.619 & 1.26 & 2.885 & 9.767 & 19.87 & 17.64 & 51.34 & 99.11 \\
\hline & Cols & 298 & 592 & 809 & 973 & 1606 & 2130 & 1842 & 2830 & 3688 \\
\hline \multirow{2}{*}{\multicolumn{2}{|c|}{$\begin{array}{l}\text { Shioda et al. } \\
\text { instances }\end{array}$}} & \multicolumn{3}{|c|}{40 commodities } & \multicolumn{3}{|c|}{60 commodities } & \multicolumn{3}{|c|}{80 commodities } \\
\hline & & $20 \mathrm{a}$ & $40 \mathrm{a}$ & $60 \mathrm{a}$ & $20 \mathrm{a}$ & $40 \mathrm{a}$ & $60 a$ & $20 \mathrm{a}$ & $40 \mathrm{a}$ & $60 \mathrm{a}$ \\
\hline \multirow[t]{2}{*}{ INIT 0} & Time & 5.51 & 7.79 & 11.39 & 43.12 & 47.04 & 56.83 & 198.38 & 221.28 & 260.09 \\
\hline & Cols & 1413 & 1714 & 2015 & 2721 & 3046 & 3506 & 4586 & 5130 & 5645 \\
\hline \multirow[t]{2}{*}{ INIT 1} & Time & 3 & 3.91 & 6.03 & 22.54 & 23.22 & 26.53 & 119.42 & 92.24 & 102.7 \\
\hline & Cols & 1138 & 1309 & 1543 & 2290 & 2463 & 2603 & 3992 & 3987 & 4181 \\
\hline \multirow{2}{*}{\multicolumn{2}{|c|}{$\begin{array}{l}\text { A1 } \\
\text { instances }\end{array}$}} & \multicolumn{3}{|c|}{21 commodities } & \multicolumn{3}{|c|}{55 commodities } & \multicolumn{3}{|c|}{91 commodities } \\
\hline & & $21 \mathrm{a}$ & $55 \mathrm{a}$ & $91 \mathrm{a}$ & $21 \mathrm{a}$ & $55 \mathrm{a}$ & $91 \mathrm{a}$ & $21 \mathrm{a}$ & $55 \mathrm{a}$ & $91 \mathrm{a}$ \\
\hline \multirow[t]{2}{*}{ INIT 0} & Time & 0.07 & 0.22 & 0.46 & 0.48 & 1.69 & 3.85 & 3.14 & 8.63 & 16.45 \\
\hline & Cols & 137 & 333 & 460 & 347 & 572 & 863 & 727 & 1185 & 1527 \\
\hline \multirow[t]{2}{*}{ INIT 1} & Time & 0.06 & 0.18 & 0.34 & 0.51 & 1.51 & 3.26 & 3.24 & 8.17 & 15.40 \\
\hline & Cols & 150 & 336 & 464 & 367 & 568 & 823 & 731 & 1153 & 1487 \\
\hline
\end{tabular}

Table 4.4: Numerical results on different initialisations for the linear relaxation of (HPDW)

In Figure 4.6 we report the performance profile graphs for the two different initialisation strategies, where the $y$-axis represents the number of instances solved within the corresponding time (in seconds) in the x-axis, such that the higher the curve the better the strategy is performing. We can see that the additional initialisation slightly improves the solution time for partial graph instances (Figure 4.6(b)) and for A1 instances 
(Figure 4.6(d) and provides a significant improvement for Shioda et al.'s instances (Figure 4.6(c)), whilst performances for complete graph instances remain the same (Figure 4.6(a)). Moreover, from Figure 4.6(a) we can notice that the solution time of complete graph instances has a very defined "step" shape: the first 70 instances are solved in less than 30 seconds each, then 10 instances are solved in 55 to 70 seconds each (these are the 90k 56a instances) and finally the biggest instances (90k 90a) are solved in 95 to 130 seconds each, for both initialisation strategies. This structure is less evident for the other three instances' types.

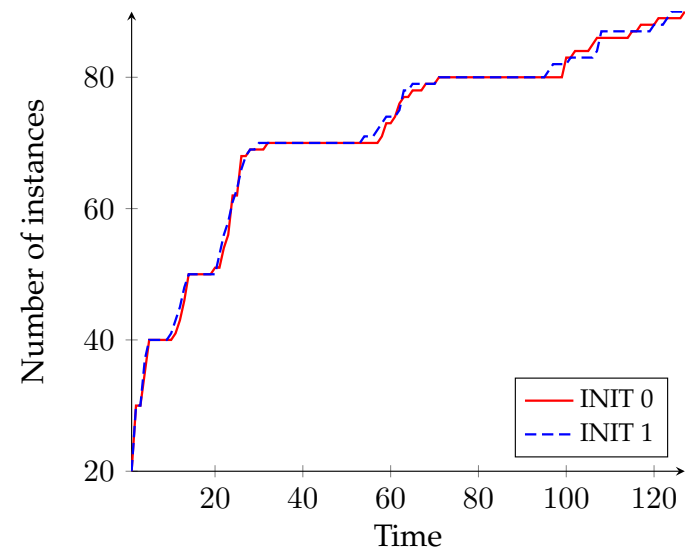

(a) Complete graph instances

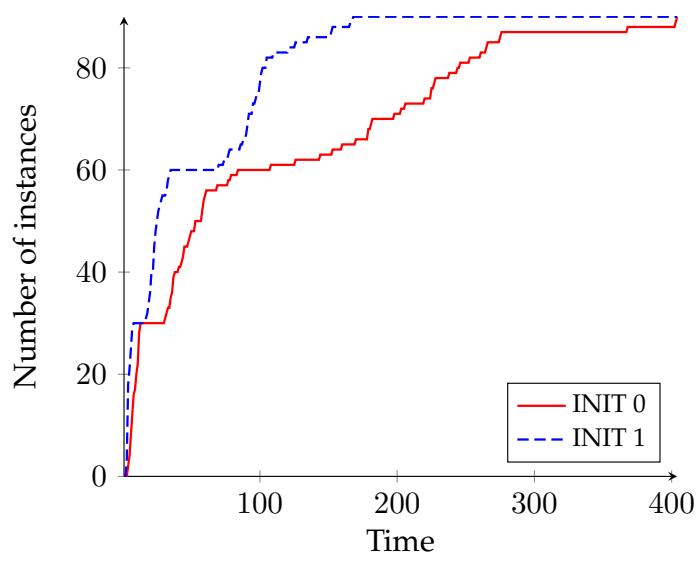

(c) Shioda et al.'s instances

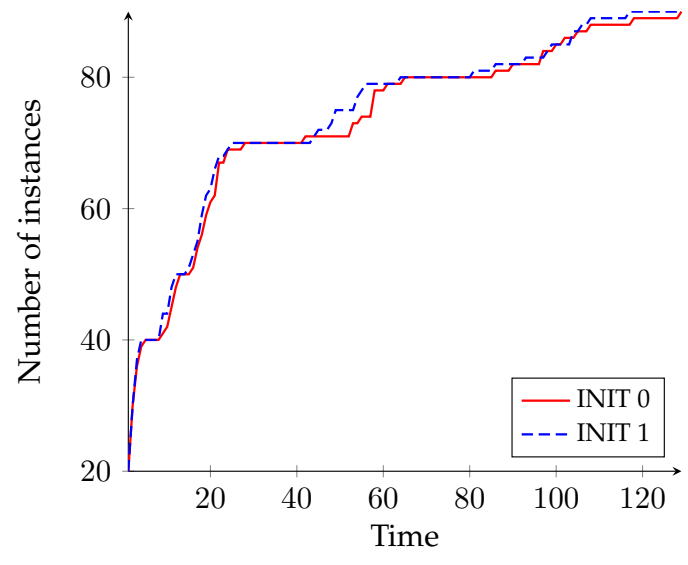

(b) Partial graph instances

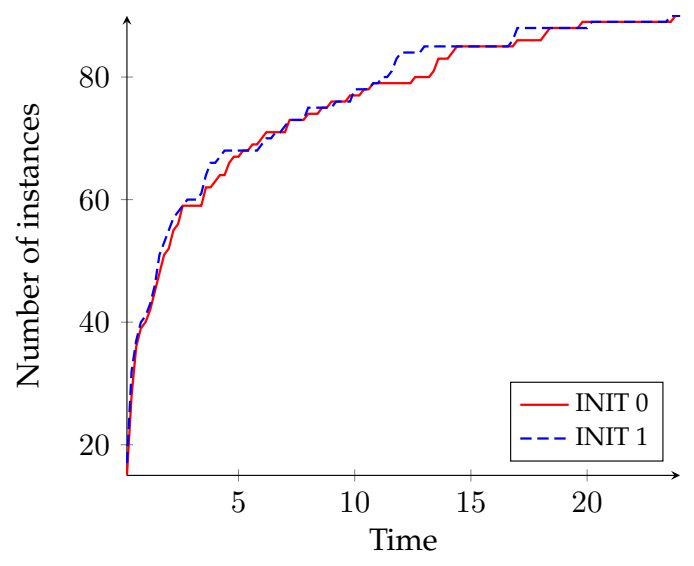

(d) A1 instances

Figure 4.6: Performance profile graphs on different initialisations for (HPDW-LR)

In Figure 4.7 we plot the evolution of the primal and dual bound during the solving of one instance with the column generation algorithm, with both initialisation strategies. The convergence of the algorithm for the complete graph instance (Figure 4.7(a) is very similar for both initialisation strategies, whilst there is an improvement using the additional initialisation for Shioda et al's instance. In Figure 4.7(b) we see that with the additional initialisation both primal and dual bounds converge quicker to the optimal value and the oscillation behaviour of the dual bound is a little bit reduced. The tailing-off effect revealed in Section 4.3.5 is also limited. 


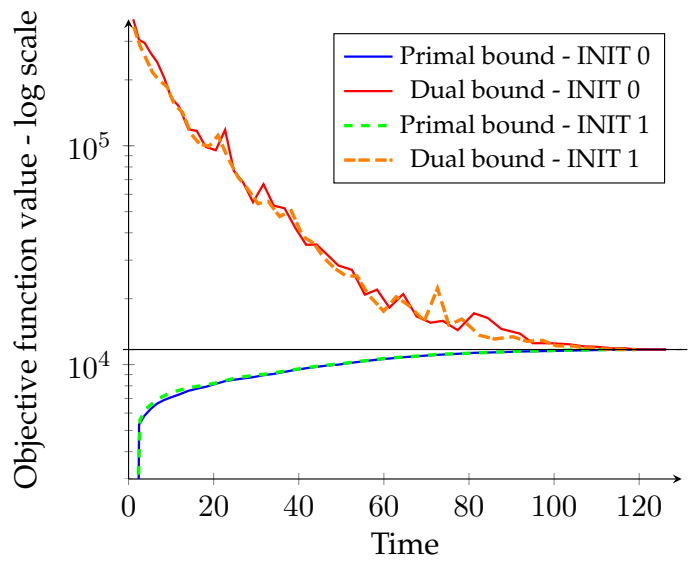

(a) 90k 90a complete graph instance

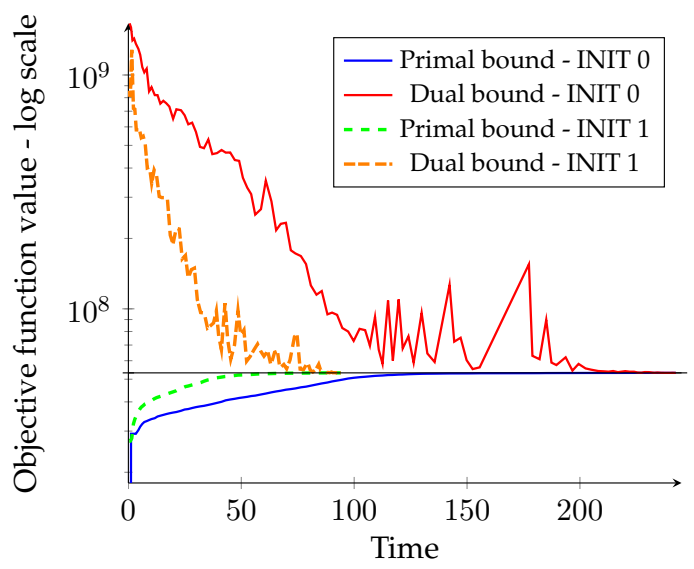

(b) $80 \mathrm{k} 60 \mathrm{a}$ Shioda et al. instance

Figure 4.7: Primal and dual bounds evolution while solving (HPDW) with column generation - Different initialisations performance

\subsubsection{Infeasibility and Farkas pricing}

Consider a linear program of the form $(P)=\max \left\{c^{T} x: A x=b, x \geq 0\right\}$. A well known fact is that, if feasible, the dual of an unfeasible linear program is unbounded. This has been formalised with the Farkas' Lemma:

Lemma 4.6.1 (Farkas' Lemma). Let $A \in \mathbb{R}^{m, n}$ and $b \in \mathbb{R}^{m}$. The linear system $A x=b$, $x \geq 0$ is feasible if and only if $y^{T} b \leq 0$ for each $y \in \mathbb{R}^{m}$ such that $y^{T} A \leq 0$.

This means that if a system is not feasible, then there exists a vector $\rho$ with $\rho^{T} A \geq 0$ and $\rho^{T} b<0$, which can be interpreted as a ray in the dual, in the direction of which the objective function decreases without limit. The infeasibility of system $A x=b, x \geq 0$, is therefore proved since there cannot exist a vector $x \geq 0$ such that $\rho^{T} A x=\rho^{T} b$. The idea of the Farkas pricing problem is to turn the unfeasible (MP) into a feasible one destroying this proof of infeasibility. To do so we need to add a variable $x \geq 0$ whose coefficient column $a(x)$ in the matrix $A$ is such that $\rho^{T} a(x)<0$. For a maximisation (MP) such a variable can be found by solving:

$$
\min _{x \geq 0}\{\rho a(x)\}=\max _{x \geq 0}\{-\rho a(x)\}
$$

which is the standard pricing problem with null cost coefficients for the objective function of (MP) and the dual ray vector instead of the dual variable vector. If such a variable is not found, we can conclude that the system is unfeasible. Dual ray values $\rho$ can be extracted from the current solution of the (MP) by the linear programming solver.

This method, even if descending from a very well known result as the Farkas' Lemma, has been introduced under the name of Farkas pricing and implemented only recently within the SCIP framework (Achterberg, 2009).

For the (HPDW) problem, the Farkas pricing problem can be formulated as follows: 


$$
\begin{array}{rlr}
\left(\mathrm{F}-\mathrm{SP}_{a}\right) & & \\
\max _{T, x, p} & -\left[\sum_{k \in \mathcal{K}} \sum_{b \in \mathcal{A}} \delta_{b}^{k}\left(p_{a}^{k}-M_{a}^{k} x_{a}^{k}\right)-\sum_{k \in \mathcal{K}} \delta_{a}^{k} T_{a}+\sum_{k \in \mathcal{K}} x_{a}^{k} \gamma^{k}+\mu_{a}\right], \\
\text { s.t } & p_{a}^{k}-M_{a}^{k} x_{a}^{k} \leq 0 & \forall k \in \mathcal{K}, \\
& p_{a}^{k}=T_{a} x_{a}^{k} & \forall k \in \mathcal{K}, \\
& x_{a}^{k} \in\{0,1\} & \forall k \in \mathcal{K}, \\
& T_{a} \geq 0, &
\end{array}
$$

where $\delta, \gamma$ and $\mu$ are the dual rays associated to (MP)'s constraints 4.10b), (4.10c) and (4.10d respectively, similarly as detailed for dual variables in Section 4.3.2 (for simplicity we use the same variable names). Dual rays are often also called Farkas dual variables. It can be solved with the same procedure of the standard pricing problem, for which optimal values of $T_{a}$ are in $\left\{M_{a}^{k}: k \in \mathcal{K}\right\} \cup\{0\}$ (see Section 4.3.2.

\subsection{Adding more columns at each iteration}

Experiments from the literature showed that for some problems adding more columns per iteration improves the performance of the algorithm, so we now explore this possibility.

The solution procedure for the (SP) introduced in Section 4.3.2 explores all the feasible solutions, such that it is straightforward to generate more than one column at each iteration of our column generation algorithm. We do not need to modify the solution procedure, but simply store the $v$ best columns with positive reduced price found for each $\left(\mathrm{SP}_{a}\right)$, where $v$ is the number of columns we want to add per iteration. Note that $v$ is bounded by the number of commodities, as detailed in the solution procedure.

Intuitively one would add more columns at the beginning of the solving, as we are still far from the optimum and the best column at this stage might reveal to be very bad afterwards, whilst the second (or third, ...) best column might reveal to be much better. On the contrary when we are close to the optimum adding the best column could be considered a safe enough option, as adding too many columns increases the dimension (and the solution time) of the (MP). It is possible to add this condition to the algorithm and modify the number of columns to add according to the solving stage, but this will be done implicitly by the column generation algorithm. If we fix the maximum number of columns to be added at each iteration, at the beginning we will probably add this number, whilst as we converge to the optimum we will add less and less columns, as the (SP) will find less solutions with a positive reduced price.

We tested our column generation algorithm adding $v$ columns per iteration for each $\left(\mathrm{SP}_{a}\right)$, with $v=1$ which is the standard algorithm, $v=2$ which adds also the second best column, $v=\lfloor|\mathcal{K}| / 4\rfloor, v=\lfloor|\mathcal{K}| / 2\rfloor, v=\lfloor 3|\mathcal{K}| / 4\rfloor$ and $v=|\mathcal{K}|$, where $|\mathcal{K}|$ is the 
number of commodities in the instance.

In Tables 4.5 and 4.6 we report numerical results on these different strategies when solving the linear relaxation of (HPDW) model, whilst tests for the integer problem are reported in Chapter 5. We report the solution time in seconds, the total number of columns generated to solve the model, and the number of iterations of the algorithm. For bigger instances of the first three classes and some choices of $v$ the column generation algorithm was interrupted by the solver for an "invalid LP", such that we were not able to obtain numerical results (indicated by - in the table).

In Figure 4.8 we report the performance profile graphs for the solution time of the different strategies for the number of columns added at each iteration of the column generation algorithm.

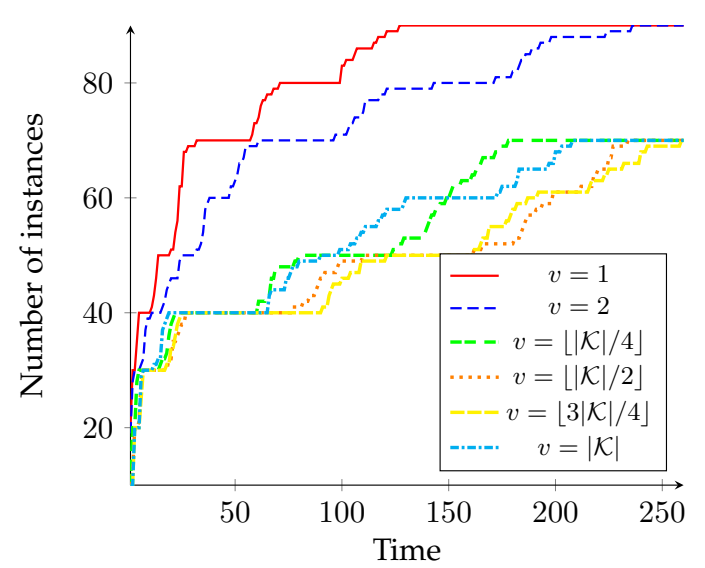

(a) Complete graph instances

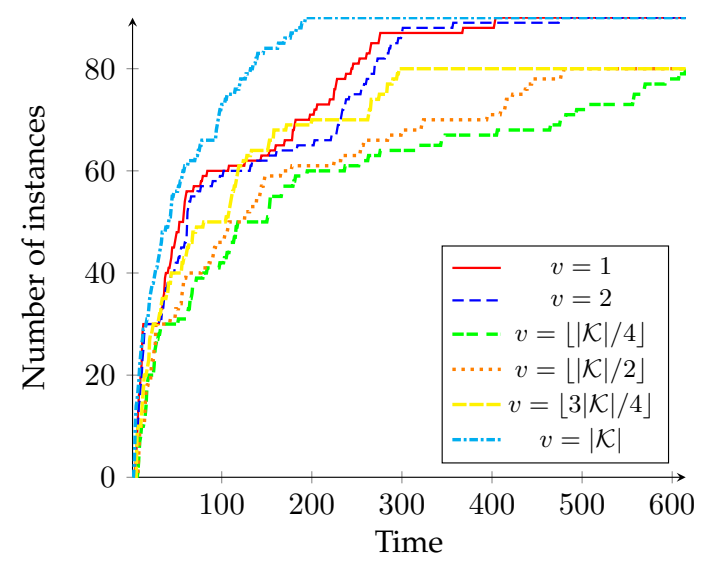

(c) Shioda et al.'s instances

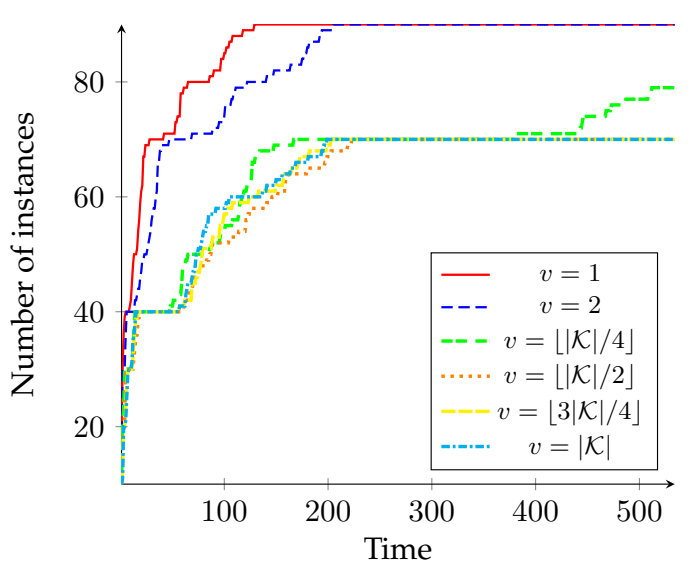

(b) Partial graph instances

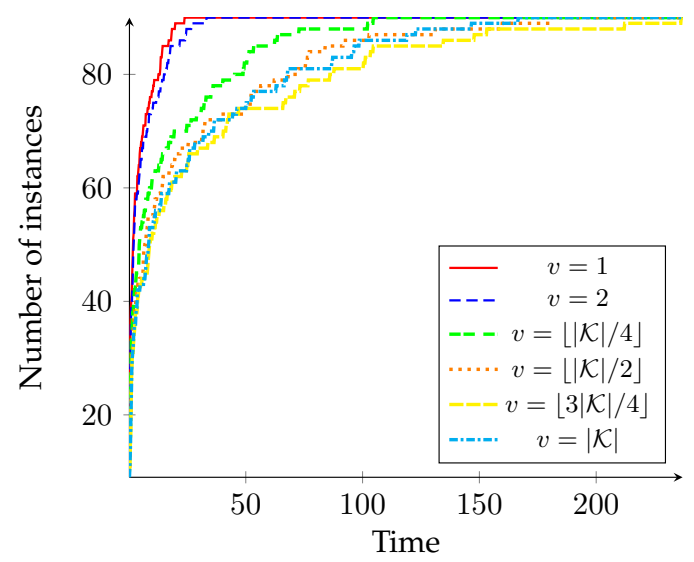

(d) A1 instances

Figure 4.8: Performance profile graphs on adding more columns at each iteration for (HPDW-LR)

We notice that only adding one or two columns per iteration allows us to solve the problem for all instances, whilst adding more columns provides numerical problems for the bigger instances of the first three classes. Only A1 instances were solved to optimality for all strategies. When the instances are solved to optimality we notice that 


\begin{tabular}{|c|c|c|c|c|c|c|c|c|c|c|}
\hline \multirow{2}{*}{$\begin{array}{l}\text { Complete } \\
\text { graph }\end{array}$} & & \multicolumn{3}{|c|}{20 commodities } & \multicolumn{3}{|c|}{56 commodities } & \multicolumn{3}{|c|}{90 commodities } \\
\hline & & $20 \mathrm{a}$ & $56 \mathrm{a}$ & $90 \mathrm{a}$ & $20 \mathrm{a}$ & $56 \mathrm{a}$ & $90 \mathrm{a}$ & $20 \mathrm{a}$ & 56 a & $90 \mathrm{a}$ \\
\hline \multirow[t]{3}{*}{$v=1$} & Time & 0.199 & 0.726 & 1.57 & 4.13 & 12.7 & 22.3 & 25.84 & 62.26 & 108.99 \\
\hline & Cols & 335 & 655 & 924 & 1141 & 1765 & 2227 & 2103 & 3034 & 3749 \\
\hline & Iters & 21 & 15 & 14 & 63 & 37 & 29 & 111 & 61 & 47 \\
\hline \multirow[t]{3}{*}{$v=2$} & Time & 0.22 & 0.84 & 1.86 & 7.77 & 19.92 & 34.93 & 52.10 & 111.91 & 194.08 \\
\hline & Cols & 556 & 1078 & 1580 & 2214 & 3363 & 4252 & 4211 & 5885 & 7420 \\
\hline & Iters & 18 & 14 & 12 & 63 & 36 & 29 & 113 & 59 & 47 \\
\hline \multirow[t]{3}{*}{$v=\lfloor|\mathcal{K}| / 4\rfloor$} & Time & 0.32 & 1.50 & 3.56 & 18.46 & 67.96 & 138.28 & 162.61 & - & - \\
\hline & Cols & 967 & 2042 & 2973 & 7285 & 12712 & 17079 & 19774 & - & - \\
\hline & Iters & 14 & 11 & 10 & 34 & 22 & 19 & 56 & - & - \\
\hline \multirow[t]{3}{*}{$v=\lfloor|\mathcal{K}| / 2\rfloor$} & Time & 0.43 & 2.23 & 5.60 & 22.19 & 90.68 & 214.87 & 187.98 & - & - \\
\hline & Cols & 1368 & 3069 & 4684 & 9694 & 18119 & 24609 & 25369 & - & - \\
\hline & Iters & 12 & 9 & 9 & 28 & 19 & 15 & 44 & - & - \\
\hline \multirow[t]{3}{*}{$v=\lfloor 3|\mathcal{K}| / 4\rfloor$} & Time & 0.44 & 2.44 & 6.05 & 21.39 & 100.82 & 228.07 & 173.26 & - & - \\
\hline & Cols & 1515 & 3394 & 5176 & 10303 & 19680 & 27557 & 26379 & - & - \\
\hline & Iters & 10 & 8 & 8 & 23 & 16 & 13 & 37 & - & - \\
\hline \multirow{3}{*}{$v=|\mathcal{K}|$} & Time & 0.37 & 2.28 & 5.66 & 15.45 & 72.90 & 189.84 & 114.20 & - & - \\
\hline & Cols & 1399 & 3194 & 4965 & 8452 & 15867 & 23276 & 20536 & - & - \\
\hline & Iters & 8 & 7 & 6 & 20 & 12 & 10 & 30 & - & - \\
\hline \multirow{2}{*}{\multicolumn{2}{|c|}{$\begin{array}{l}\text { Partial } \\
\text { graph }\end{array}$}} & \multicolumn{3}{|c|}{20 commodities } & \multicolumn{3}{|c|}{56 commodities } & \multicolumn{3}{|c|}{90 commodities } \\
\hline & & $20 \mathrm{a}$ & $56 \mathrm{a}$ & $90 \mathrm{a}$ & $20 \mathrm{a}$ & $56 \mathrm{a}$ & $90 \mathrm{a}$ & $20 \mathrm{a}$ & $56 \mathrm{a}$ & $90 \mathrm{a}$ \\
\hline \multirow[t]{3}{*}{$v=1$} & Time & 0.171 & 0.687 & 1.443 & 3.026 & 10.99 & 21.23 & 18.54 & 55.71 & 102.87 \\
\hline & Cols & 301 & 625 & 891 & 974 & 1699 & 2199 & 1853 & 2926 & 3747 \\
\hline & Iters & 19 & 15 & 13 & 55 & 35 & 29 & 100 & 58 & 47 \\
\hline \multirow[t]{3}{*}{$v=2$} & Time & 0.17 & 0.77 & 1.66 & 4.84 & 17.87 & 31.36 & 35.43 & 99.77 & 175.37 \\
\hline & Cols & 506 & 1051 & 1500 & 1843 & 3250 & 4068 & 3615 & 5694 & 7270 \\
\hline & Iters & 17 & 13 & 12 & 53 & 34 & 27 & 97 & 57 & 46 \\
\hline \multirow[t]{3}{*}{$v=\lfloor|\mathcal{K}| / 4\rfloor$} & Time & 0.29 & 1.36 & 3.15 & 12.41 & 57.81 & 123.93 & 111.57 & 468.91 & - \\
\hline & Cols & 888 & 1883 & 2791 & 6176 & 12095 & 16411 & 17078 & 30479 & - \\
\hline & Iters & 13 & 10 & 10 & 29 & 21 & 18 & 50 & 31 & - \\
\hline \multirow[t]{3}{*}{$v=\lfloor|\mathcal{K}| / 2\rfloor$} & Time & 0.34 & 1.90 & 4.50 & 15.09 & 71.48 & 183.72 & 117.83 & - & - \\
\hline & Cols & 1200 & 2698 & 3901 & 8082 & 16255 & 22435 & 21364 & - & - \\
\hline & Iters & 11 & 9 & 8 & 25 & 17 & 15 & 37 & - & - \\
\hline \multirow[t]{3}{*}{$v=\lfloor 3|\mathcal{K}| / 4\rfloor$} & Time & 0.37 & 2.17 & 5.48 & 13.42 & 71.88 & 168.73 & 95.97 & - & - \\
\hline & Cols & 1316 & 2975 & 4690 & 8111 & 16296 & 22752 & 19926 & - & - \\
\hline & Iters & 10 & 8 & 7 & 20 & 14 & 12 & 30 & - & - \\
\hline \multirow[t]{3}{*}{$v=|\mathcal{K}|$} & Time & 0.38 & 2.36 & 5.94 & 12.48 & 67.84 & 168.30 & 84.43 & - & - \\
\hline & Cols & 1342 & 3272 & 4962 & 7725 & 16550 & 22895 & 18861 & - & - \\
\hline & Iters & 9 & 8 & 7 & 19 & 13 & 11 & 29 & - & - \\
\hline
\end{tabular}

Table 4.5: Numerical results on the number of columns added at each iteration for the linear relaxation of (HPDW) Complete and partial graph instances

adding more columns reduces the number of iterations of the algorithms and increases the number of generated columns. For complete and partial graph and A1 instances adding the best column at each iteration is the best strategy. Adding also the second best column does not reduce a lot the number of iterations whilst the number of gener- 


\begin{tabular}{|c|c|c|c|c|c|c|c|c|c|c|}
\hline \multirow{2}{*}{$\begin{array}{l}\text { Shioda et al. } \\
\text { instances }\end{array}$} & & \multicolumn{3}{|c|}{40 commodities } & \multicolumn{3}{|c|}{60 commodities } & \multicolumn{3}{|c|}{80 commodities } \\
\hline & & $20 \mathrm{a}$ & $40 \mathrm{a}$ & $60 \mathrm{a}$ & $20 \mathrm{a}$ & $40 \mathrm{a}$ & $60 \mathrm{a}$ & $20 \mathrm{a}$ & $40 \mathrm{a}$ & $60 \mathrm{a}$ \\
\hline \multirow[t]{3}{*}{$v=1$} & Time & 5.51 & 7.79 & 11.39 & 43.12 & 47.04 & 56.83 & 198.38 & 221.28 & 260.09 \\
\hline & Cols & 1413 & 1714 & 2015 & 2721 & 3046 & 3506 & 4586 & 5130 & 5645 \\
\hline & Iters & 82 & 50 & 40 & 165 & 91 & 69 & 277 & 153 & 110 \\
\hline \multirow[t]{3}{*}{$v=2$} & Time & 5.89 & 8.85 & 12.82 & 38.56 & 55.90 & 73.26 & 212.24 & 238.50 & 297.67 \\
\hline & Cols & 2096 & 2636 & 3194 & 3838 & 4785 & 5457 & 6825 & 7716 & 8719 \\
\hline & Iters & 63 & 40 & 32 & 119 & 71 & 54 & 216 & 115 & 87 \\
\hline \multirow[t]{3}{*}{$v=\lfloor|\mathcal{K}| / 4\rfloor$} & Time & 8.63 & 16.27 & 27.44 & 65.75 & 107.05 & 165.30 & 353.68 & 539.27 & - \\
\hline & Cols & 4823 & 7009 & 8926 & 11649 & 15958 & 19847 & 23030 & 31433 & - \\
\hline & Iters & 34 & 24 & 21 & 58 & 38 & 30 & 86 & 55 & - \\
\hline \multirow[t]{3}{*}{$v=\lfloor|\mathcal{K}| / 2\rfloor$} & Time & 8.10 & 16.15 & 24.74 & 53.53 & 94.60 & 140.44 & 262.96 & 433.35 & - \\
\hline & Cols & 6014 & 8977 & 10858 & 13748 & 20181 & 24825 & 26769 & 37867 & - \\
\hline & Iters & 25 & 18 & 14 & 38 & 27 & 21 & 60 & 37 & - \\
\hline \multirow[t]{3}{*}{$v=\lfloor 3|\mathcal{K}| / 4\rfloor$} & Time & 5.63 & 12.07 & 19.78 & 36.56 & 63.95 & 113.60 & 147.21 & 277.93 & - \\
\hline & Cols & 5610 & 8685 & 10830 & 12501 & 18520 & 22893 & 22646 & 32840 & - \\
\hline & Iters & 18 & 14 & 11 & 29 & 19 & 15 & 41 & 27 & - \\
\hline \multirow[t]{3}{*}{$v=|\mathcal{K}|$} & Time & 3.25 & 6.14 & 10.96 & 21.07 & 33.23 & 46.85 & 84.52 & 111.65 & 157.44 \\
\hline & Cols & 3964 & 5084 & 5873 & 9112 & 12292 & 12819 & 16382 & 20158 & 23484 \\
\hline & Iters & 15 & 9 & 8 & 22 & 13 & 10 & 33 & 18 & 13 \\
\hline \multirow{2}{*}{\multicolumn{2}{|c|}{$\begin{array}{l}\text { A1 } \\
\text { instances }\end{array}$}} & \multicolumn{3}{|c|}{21 commodities } & \multicolumn{3}{|c|}{55 commodities } & \multicolumn{3}{|c|}{91 commodities } \\
\hline & & $21 \mathrm{a}$ & $55 \mathrm{a}$ & $91 \mathrm{a}$ & $21 \mathrm{a}$ & $55 \mathrm{a}$ & $91 \mathrm{a}$ & $21 \mathrm{a}$ & $55 \mathrm{a}$ & $91 \mathrm{a}$ \\
\hline \multirow[t]{3}{*}{$v=1$} & Time & 0.07 & 0.22 & 0.46 & 0.48 & 1.69 & 3.85 & 3.14 & 8.63 & 16.45 \\
\hline & Cols & 137 & 333 & 460 & 347 & 572 & 863 & 727 & 1185 & 1527 \\
\hline & Iters & 11 & 12 & 11 & 30 & 21 & 19 & 59 & 39 & 29 \\
\hline \multirow[t]{3}{*}{$v=2$} & Time & 0.07 & 0.28 & 0.56 & 0.63 & 2.02 & 4.39 & 4.03 & 10.62 & 21.20 \\
\hline & Cols & 203 & 489 & 658 & 539 & 913 & 1321 & 1198 & 2016 & 2585 \\
\hline & Iters & 10 & 9 & 8 & 25 & 18 & 15 & 48 & 31 & 26 \\
\hline \multirow[t]{3}{*}{$v=\lfloor|\mathcal{K}| / 4\rfloor$} & Time & 0.08 & 0.44 & 0.78 & 1.16 & 4.32 & 10.76 & 12.44 & 30.87 & 64.50 \\
\hline & Cols & 309 & 824 & 986 & 1655 & 2925 & 4557 & 6032 & 10305 & 13795 \\
\hline & Iters & 8 & 8 & 7 & 17 & 13 & 11 & 31 & 22 & 19 \\
\hline \multirow[t]{3}{*}{$v=\lfloor|\mathcal{K}| / 2\rfloor$} & Time & 0.09 & 0.60 & 1.12 & 1.71 & 6.26 & 15.73 & 16.02 & 49.08 & 103.47 \\
\hline & Cols & 436 & 1201 & 1429 & 2733 & 4704 & 7410 & 10228 & 18316 & 24069 \\
\hline & Iters & 8 & 7 & 7 & 17 & 12 & 11 & 30 & 22 & 18 \\
\hline \multirow[t]{3}{*}{$v=\lfloor 3|\mathcal{K}| / 4\rfloor$} & Time & 0.11 & 0.67 & 1.31 & 2.13 & 8.36 & 20.75 & 18.39 & 62.58 & 136.53 \\
\hline & Cols & 528 & 1420 & 1751 & 3823 & 6519 & 10194 & 13543 & 24741 & 33400 \\
\hline & Iters & 8 & 7 & 7 & 16 & 13 & 10 & 28 & 20 & 17 \\
\hline \multirow[t]{3}{*}{$v=|\mathcal{K}|$} & Time & 0.11 & 0.69 & 1.34 & 2.05 & 7.94 & 19.47 & 16.89 & 50.59 & 108.60 \\
\hline & Cols & 539 & 1418 & 1822 & 3726 & 6316 & 9700 & 12746 & 21655 & 29511 \\
\hline & Iters & 7 & 6 & 6 & 14 & 10 & 9 & 22 & 15 & 12 \\
\hline
\end{tabular}

Table 4.6: Numerical results on the number of columns added at each iteration for the linear relaxation of (HPDW) Shioda et al. and A1 instances

ated columns increases, such that the solution time is bigger. For Shioda et al. instances results are slightly different, as adding $|\mathcal{K}|$ columns per iteration allows us to obtain the best results: in fact the number of iterations becomes quite small such that, even if there are more columns, the solution time is reduced. 
In Figure 4.9 we plot the evolution of the primal and dual bound during the solving of one instance with the column generation algorithm, for strategies which do not lead to numerical problems $(v=1,2$ for the complete graph instance, $v=1,2,|\mathcal{K}|$ for Shioda et al. instances). In addition to confirming what previously said, further considerations can be made: strategy $v=2$ has a bigger solution time, but the dual bound value has less oscillations, and for Shioda et al. instances (Figure 4.9(b)) each iteration of strategy $v=|\mathcal{K}|$ provides a big change in the bounds, especially at the beginning of the solving, resulting in no oscillations at all.

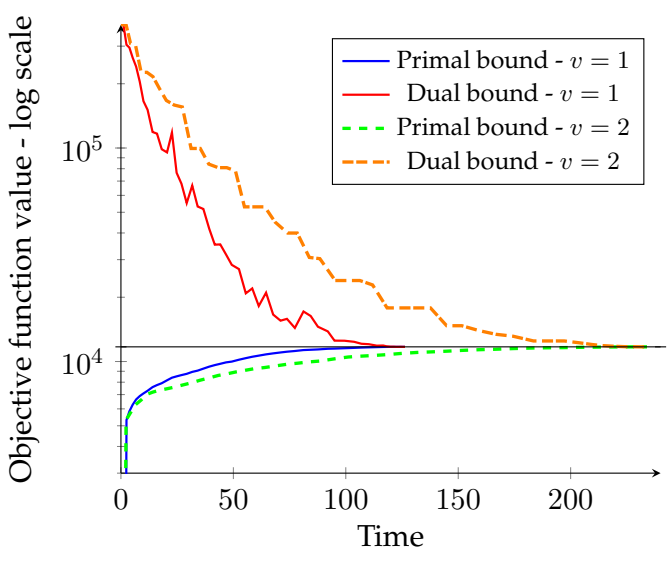

(a) 90k 90a complete graph instance

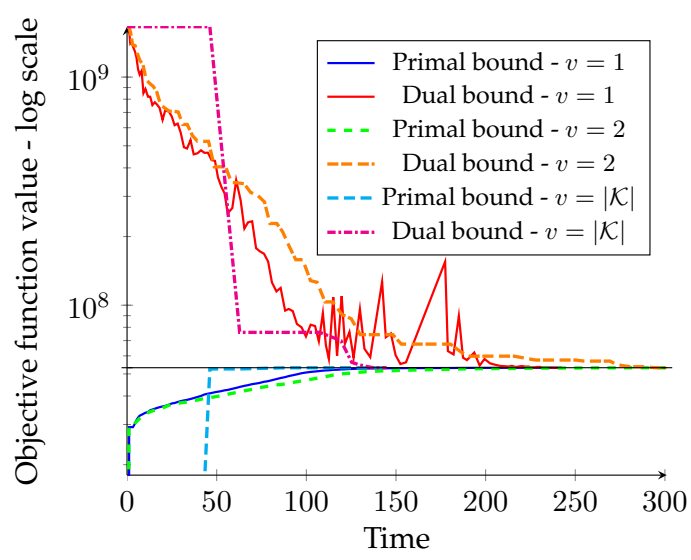

(b) $80 \mathrm{k} 60 \mathrm{a}$ Shioda et al. instance

Figure 4.9: Primal and dual bounds evolution while solving (HPDW) with column generation - Adding more columns per iteration performance

\subsection{Additional stopping criterion using dual bound}

The dual upper bound introduced in Section 4.3.3 can be integrated as an additional stopping criterion for column generation: when this dual upper bound is equal to the primal lower bound we are guaranteed to have reached optimality for the relaxed (MP) and we can stop generating columns. The primal lower bound is equal to the solution of the current (MP) and can be extracted by the LP solver. As we saw from Figure 4.2 . the dual bound oscillate when converging to the optimal value. For this reason if we keep track of the best dual bound found during the solving process and we compare it to the current primal bound (which does not oscillate but is non-decreasing), we might be able to stop column generation before reaching all non-positive reduced prices. This could help to reduce the tailing-off effect introduced in Section 4.3.5.

Furthermore, when solving the integer problem in the branch-and-bound tree, this dual upper bound provides another stopping criterion. This will be explained in more details in Chapter 5

We tested adding this stopping criterion when solving the linear relaxation of (HPDW) formulation, but the performance remains basically constant. Therefore, as they are not 
significant, we do not report numerical results.

This dual upper bound stopping criterion, which may not be very useful by itself, is integrated in the stabilisation procedure described in Section 4.9.2

\subsection{Stabilisation procedure for solving the (MP) with column generation}

In this section we consider a stabilisation procedure to speed up the convergence of column generation when solving the relaxed (MP). This arises from observations of the fact that dual variables do not smoothly converge to their respective optima, but oscillate, without an observed regular pattern (Lübbecke and Desrosiers, 2005). We observed this oscillation behaviour also in previous numerical results (for instance in Figure 4.1). Those oscillations can lead to "extreme columns" that have low chances of being in the optimal solution. Furthermore, columns that will be in the optimal solution are often generated in the last iterations, when dual variables are close to their optimal values (du Merle et al., 1999).

We consider two possible approaches and algorithms to limit this oscillation behaviour. The first one has been proposed by du Merle et al. (1999) and relies on boxes in which dual variables are constrained to lie in, from the principle of the Boxtep method introduce by Marsten (1975) and Marsten et al. (1975). The second one is a smoothing approach for dual variables, proposed by Pessoa et al. (2010) on ideas from Wentges (1997).

\subsubsection{Boxtep method with slack variables}

This stabilised column generation approach considers boxes in which dual variables are constrained to lie, and imposes some penalisation factors if they are outside.

Consider a problem $(\mathrm{P})$ of the form $\min \left\{c^{T} x: A x=b, x \geq 0\right\}$. We perturb (P) adding surplus and slack variables $y_{-}$and $y_{+}$, bounded by $\epsilon_{-}$and $\epsilon_{+}$and penalised by $\omega_{-}$and $\omega_{+}$in the objective function, respectively:

$$
\begin{aligned}
\min _{\tilde{x}, y_{-}, y_{+}} & \\
\text {s.t. } & c^{T} \tilde{x}-\omega_{-}^{T} y_{-}+\omega_{+}^{T} y_{+} \\
& A \tilde{x}-y_{-}+y_{+}=b \\
& y_{-} \leq \epsilon_{-} \\
& y_{+} \leq \epsilon_{+} \\
& \tilde{x}, y_{-}, y_{+} \geq 0
\end{aligned}
$$

The dual of this perturbed problem is therefore: 
$(\tilde{D})$

$$
\begin{aligned}
\max _{\tilde{\pi}, w_{-}, w_{+}} & b^{T} \tilde{\pi}-\epsilon_{-}^{T} \psi_{-}-\epsilon_{+}^{T} \psi_{+} \\
\text {s.t. } & A^{T} \tilde{\pi} \leq c \\
& -\tilde{\pi}-\psi_{-} \leq-\omega_{-} \\
& \tilde{\pi}-\psi_{+} \leq \omega_{+} \\
& \psi_{-}, \psi_{+} \geq 0
\end{aligned}
$$

where dual variables $\pi$ are associated to constraints 4.43c), $\psi_{-}$to constraints $4.43 \mathrm{~d}$ and $\psi_{+}$to constraints $4.43 \mathrm{e}$ of $(\tilde{P})$.

Constraints $4.44 \mathrm{~d}$ and $4.44 \mathrm{e}$ of $(\tilde{D})$ can be rewritten as $\omega_{-}-\psi_{-} \leq \tilde{\pi} \leq \omega_{+}+\psi_{+}$, giving the box in which dual variables $\tilde{\pi}$ are constraint to lie in: $\left[\omega_{-}-\psi_{-}, \omega_{+}+\psi_{+}\right]$.

The following proposition provides the conditions in which the perturbed problem equals the original problem, which will be used as stopping criteria for the stabilisation algorithm.

Proposition 4.9.1 $((\tilde{P}) \equiv(P)$, from du Merle et al. (1999)). The perturbed problem $(\tilde{P})$ is equivalent to the original problem $(P)$, i.e. $y_{-}^{*}=y_{+}^{*}=0$ where $y_{-}^{*}, y_{+}^{*}$ are optimal solutions of $(\tilde{P})$, if one of the two following conditions is met: (i) $\epsilon_{-}=\epsilon_{+}=0$ or (ii) $\omega_{-}<\tilde{\pi}^{*}<\omega_{+}$, where $\tilde{\pi}^{*}$ is the optimal solution of $(\tilde{D})$.

Proof. If (i) is verified then $y_{-}=y_{+}=0$ from constraints 4.43d, 4.43e and 4.43f), and this is valid also for optimal solutions $y_{-}^{*}=y_{+}^{*}=0$. If (ii) holds then from the complementary slackness conditions of primal variables $y_{-}$and $y_{+}$and dual constraints 4.44d and 4.44e we conclude that $y_{-}^{*}=y_{+}^{*}=0$.

Moreover du Merle et al. (1999) show that $v(\tilde{P}) \leq b^{T} \tilde{\pi}^{*} \leq v(P)$, meaning that $b^{T} \tilde{\pi}^{*}$ can be a better bound on $v(P)$ than $v(\tilde{P})$.

We will now detail the algorithm they proposed applying it to our problem.

\section{Boxtep method for (HPDW)}

We add slack variables to allow a small violation of shortest path constraints and penalise it in the objective function. We introduce a set of variables $y_{b}^{k}$ that takes into account the violation of constraints $4.10 \mathrm{~b}$. These variables are positive and limited by a set of parameters $\epsilon_{b}^{k}$ that are updated during the procedure. They are penalised in the objective function with a set of parameters $\omega_{b}^{k}$ also updated during the procedure.

The linear relaxation of the perturbed (MP) can be written as follows: 


$$
\begin{array}{lll}
\max _{\lambda}^{(M P)} & \sum_{a \in \mathcal{A}} \sum_{k \in \mathcal{K}} \sum_{j \in \mathcal{J}} \lambda_{a}^{j} \eta^{k} p_{a}^{k, j}-\sum_{b \in \mathcal{A}} \sum_{k \in \mathcal{K}} \omega_{b}^{k} y_{b}^{k}, & \\
\text { s.t. } & \sum_{a \in \mathcal{A}} \sum_{j \in \mathcal{J}} \lambda_{a}^{j}\left(p_{a}^{k, j}-M_{a}^{k} x_{a}^{k, j}\right)-\sum_{j \in \mathcal{J}} \lambda_{b}^{j} T_{b}^{j}-y_{b}^{k} \leq-M_{b}^{k} & \forall k \in \mathcal{K}, \forall b \in \mathcal{A}, \\
& \sum_{a \in \mathcal{A}} \sum_{j \in \mathcal{J}} \lambda_{a}^{j} x_{a}^{k, j} \leq 1 & \forall k \in \mathcal{K}, \\
& \sum_{j \in \mathcal{J}} \lambda_{a}^{j}=1 & \forall a \in \mathcal{A}, \\
& \lambda_{a}^{j} \geq 0 & \forall j \in \mathcal{J}, \forall a \in \mathcal{A}, \\
& 0 \leq y_{b}^{k} \leq \epsilon_{b}^{k} & \forall b \in \mathcal{A}, \forall k \in \mathcal{K} .
\end{array}
$$

The formulation and solution procedure of the subproblem are not modified, as nothing added affect priced variables $\lambda$, but subproblem solutions will be different depending on the value of $y$ variables at each iteration. If we look at the dual of $(\tilde{M P})$ and at the constraints associated to $y$ variables, we can see that:

$$
0 \leq \delta_{b}^{k} \leq \omega_{b}^{k}+\psi_{b}^{k}
$$

where $\psi_{b}^{k}$ are dual variables associated to constraints $4.45 \mathrm{f}$. With the perturbation, we constrain dual variables $\delta_{b}^{k}$ into "a box".

\section{Algorithm for column generation and updating parameters}

The criterion for having an optimal $(\tilde{M} P)$ is having all non-positive reduced prices from the subproblem and at the same time having reached the stopping criterion for the boxtep method. This means that if we cannot find new columns to improve the $(\tilde{M P})$ but the stopping criterion of the box method is not reached, we need to update $\epsilon$ and $\omega$ parameters and solve again the $(\tilde{M P})$.

The stopping criterion of the boxtep method is, for all $b \in \mathcal{A}$ and $k \in \mathcal{K}$ :

$$
\text { (i) } \epsilon_{b}^{k}=0 \quad \text { or } \quad \text { (ii) } 0 \leq \delta_{b}^{k}<\omega_{b}^{k} \text {. }
$$

Let us introduce an iteration index $i$ for the column generation algorithm, such that $\epsilon_{b}^{k, i}$ and $\omega_{b}^{k, i}$ are the perturbation parameters at iteration $i$. First of all we need to initialise the $\epsilon$ and $\omega$ parameters:

- $\epsilon_{b}^{k, 0}$ should be set to any value to be tuned during numerical tests; 
- $\omega_{b}^{k, 0}$ should be set to any value, possibly a good estimate of optimal dual variables.

Further, we need to update the parameters at each iteration of column generation $i$ :

- if $U B^{i}$ is the best known estimate of the dual optimal value at iteration $i$ (best know dual upper bound) or we reached optimality for standard column generation (all non-positive reduced prices) we then decrease values of $\epsilon_{b}^{k, i+1}$, otherwise we increase these values;

- if $U B^{i}$ is the best known estimate of the dual optimal value, we update $\omega_{b}^{k, i+1}=$ $\delta_{b}^{k, i}$, otherwise we do not update them.

Finally, after a certain number of iterations:

- we always decrease $\epsilon_{b}^{k, i+1}$ such that they become zero in a finite number of iterations,

- and only if we reached optimality for standard column generation (all non-positive reduced prices), but the stopping criterion of boxtep method is not reached yet, we then update $\omega_{b}^{k, i+1}=\delta_{b}^{k, i}$.

Initial values and criterion for updating parameters (e.g. increasing and decreasing rules) should be chosen performing numerical tests on different possibilities, as they depend on the specific problem and general rules to follow do not seem to be known.

As described at the beginning of the chapter, all our tests have been implemented using SCIP framework (Achterberg, 2009), where it is not possible to relax (i.e. increase for our case) the bound of variables and change the parameters in the objective function during the resolution. Furthermore it is possible to continue the column generation only if a new column has been added, such that we are not able to continue the solving to fulfil criterion (4.47) once the reduced price of all variables is non positive. For these reasons we were not able to implement this algorithm for our problem and we cannot give computational results for this procedure and good choices for parameters and updating rules.

\subsubsection{Dual variable smoothing approach}

A dual variable smoothing approach stabilisation procedure to speed-up column generation has been introduced by Wentges (1997) and further developed by Pessoa et al. (2010). It differs from the boxtep method described above by its simplicity, as there is only one parameter and updating rule involved. The basic idea is that at each iteration of the column generation procedure, instead of using current dual values, we use a convex combination of these current values with the best known estimates. Whenever we find better dual values, we update the best estimates. To guarantee the convergence with the correct optimal solution, the last iterations are done with the standard column generation without any stabilisation. 
Consider a scalar parameter $\Delta$, such that $0<\Delta \leq 1$. Let $\bar{\pi}$ be the current best know vector of dual multipliers, which is the vector providing the smallest (dual) upper bound (for a maximisation problem) among all vectors that have been evaluated. Let $\pi_{M P}$ be the current solution of dual multipliers of the restricted (MP) on a certain iteration of column generation. Instead of using $\pi_{M P}$ when solving the next subproblem, we solve it using a convex combination of these values, as follows:

$$
\pi=\Delta \pi_{M P}+(1-\Delta) \bar{\pi}
$$

Moreover, if this vector of dual multipliers $\pi$ improves the dual upper bound, we update the best know vector $\bar{\pi}=\pi$.

Let $U B(\bar{\pi})$ be the best (smallest) upper bound found so far, calculated from the dual vector using equation (4.18), and $Z_{M P}$ the current primal lower bound, which is the current solution value of the (MP). We have found the optimal solution for (MP) when:

$$
g a p=\frac{U B(\bar{\pi})-Z_{M P}}{U B(\bar{\pi})}<\varepsilon,
$$

where $\varepsilon \geq 0$ and sufficiently small. This formalises the stopping criterion for column generation introduced in Section 4.8 .

The convergence of this procedure is guaranteed by the two following lemmas.

Lemma 4.9.2 (from Wentges (1997)). If the solution of the subproblem solved with vector $\pi$ gives a column that already exists in the current $(M P)$, then $U B(\pi) \leq U B(\bar{\pi})-\Delta(U B(\bar{\pi})-$ $\left.Z_{M P}\right)$.

This lemma guarantees that the new dual solution is better than the old one of at least a factor of $\Delta\left(U B(\bar{\pi})-Z_{M P}\right)$.

Lemma 4.9.3 (from Pessoa et al. (2010)). If the solution of the subproblem solved with vector $\pi$ does not give a column with positive reduced price with respect to vector $\pi_{M P}$ (called a "misprice"), then $U B(\pi) \leq U B(\bar{\pi})-\Delta\left(U B(\bar{\pi})-Z_{M P}\right)$.

Each misprice guarantees that the gap is reduced by at least a factor of $1 /(1-\Delta)$, such that the total number of misprices is polynomially bounded.

To avoid the need of estimating a proper value for $\varepsilon$ that guarantees optimality due to misprices, if $\operatorname{gap} \leq \bar{\varepsilon}$ (where $\bar{\varepsilon}>\varepsilon$ ) we set $\Delta=1$ such that the last iterations are done with the standard column generation without stabilisation. As at this stage we will be close to the optimal solution, dual variable values will be close to the optimal ones and the stabilisation would not change much the convergence of column generation, which should be fast.

The previous lemmas guarantee convergence even when misprices occur, but the convergence can be slow. To avoid this inconvenient if an iteration of the algorithm with the stabilisation does not improve the gap, we disable the stabilisation $(\Delta=1)$ for the following iteration. 
Performances of the stabilisation approach highly depend on a good choice of the parameter $\Delta$, and this can vary significantly for each instance. To avoid the need of tuning the algorithm Pessoa et al. (2013) proposed an auto-regulating strategy which is based on the gradient information.

We tested several strategies of defining and updating $\Delta$ parameter, which are now described. Numerical results are reported in Section 4.9 .3 .

\section{Strategy 1}

We update the parameter $\Delta$ accordingly to the current gap between the lower and upper bound of the (MP) (equation 4.49).

We choose an initial value for $\left.\left.\Delta, \Delta_{\text {init }} \in\right] 0,1\right]$ by tuning it within numerical tests. We choose also a value $\bar{\varepsilon}$ after which we stop the stabilisation.

Therefore our strategy is:

- $\Delta=\Delta_{\text {init }}$;

- if $g a p<\left(1-\Delta_{\text {init }}\right)$ then $\Delta=1-g a p$;

- if gap $<\bar{\varepsilon}$ then $\Delta=1$.

As the gap is decreasing during the solving of (MP), in this strategy the parameter $\Delta$ is always increasing: at the beginning more weight is given to the best known dual values whilst at the end more weight is given to the current ones. This strategy follows the intuition that at the beginning current dual values are more unstable and we need to stabilise more, whilst during the solving they converge to the optimum and less stabilisation is needed.

\section{Strategy 2}

This strategy was proposed by Pessoa et al. (2010), to define and update $\Delta$ during the process of solving (MP) with column generation, along a branching tree used to solve the integer problem.

Let us set $Z_{I N T}$ to the value of the current best integer solution found when solving of the integer (MP). If the solving of current restricted relaxed (MP) is at an early stage $\left(Z_{M P}<Z_{I N T}\right)$, then they use an aggressive approach, giving more weight to the best known dual variables:

$$
\Delta=0.1 \frac{U B_{\bar{\pi}}-Z_{I N T}}{U B_{\bar{\pi}}-Z_{M P}},
$$

otherwise they set $\Delta=0.1$.

The stabilisation stops when $g a p<\bar{\varepsilon}$, fixing $\Delta=1$ for this case. 


\section{Strategy 3}

We propose a mixed strategy, using the more aggressive approach for the early stage of solving (MP) in the tree of strategy 1 and the update of the parameter while solving the linear relaxation accordingly to the gap of strategy 2 . The strategy works as follows:

- $\Delta=\Delta_{\text {init }}$;

- if $Z_{M P}<Z_{I N T}$ then $\Delta=0.1 \frac{U B_{\bar{\pi}}-Z_{I N T}}{U B_{\bar{\pi}}-Z_{M P}}$;

- else if $g a p<\left(1-\Delta_{\text {init }}\right)$ then $\Delta=1-$ gap;

- if $g a p<\bar{\varepsilon}$ then $\Delta=1$.

\section{Strategy 4}

A very simple strategy is to set $\Delta$ equal to a fixed value $\Delta_{f i x}$, and disable stabilisation when the gap gets small (if gap $<\bar{\varepsilon}$ then $\Delta=1$ ). The best value of $\Delta_{\text {fix }}$ should be decided accordingly to numerical results.

\section{Strategy 5}

Following Pessoa et al. (2013) idea of an auto-adaptive scheme to choose $\Delta$, we do not tune the parameter's choice a priori, but we let the column generation algorithm modify it during the solving. They propose to use the subgradient information to update the parameter: if the subgradient reveals that the best known dual vector is good, we give more weight to it, otherwise we give more weight to the current dual vector. The strategy works as follows (for more details we refer to the paper by Pessoa et al.):

- if $g(\pi)\left(\bar{\pi}-\pi_{M P}\right)>0$ then $\Delta=f_{\text {decr }}(\Delta)$;

- else $\Delta=f_{\text {incr }}(\Delta)$;

where $g(\pi)$ is the subgradient calculated in $\pi$, and increasing and decreasing functions are as follows:

$$
\begin{gathered}
f_{\text {incr }}(\Delta)=\Delta+0.1(1-\Delta) \\
f_{\text {decr }}(\Delta)= \begin{cases}\Delta / 1.1 & \text { if } \Delta \in[0.5,1] \\
\max \{0.001, \Delta-0.1(1-\Delta)\} & \text { otherwise }\end{cases}
\end{gathered}
$$

As for the other strategies we set $\Delta=1$ if gap $<\bar{\varepsilon}$.

\subsubsection{Numerical results}

In Tables 4.7, 4.8, 4.9 and 4.10 we report numerical results for solving the linear relaxation of (HPDW) model with the different stabilisation strategies of the dual variable smoothing approach. The time in seconds and the number of generated columns are reported. As we are solving the linear relaxation, Strategy 2 is reduced to Strategy 4 
with a $\Delta_{f i x}=0.1$. Similarly, Strategy 3 is reduced to Strategy 1 . We consider the model without any stabilisation (no stab), with the Strategy 1 and different values for $\Delta_{\text {init }}$ and $\bar{\varepsilon}$ (Str $1-\Delta_{\text {init }}, \bar{\varepsilon}$ ), with the Strategy 4 and different values for $\Delta_{f i x}$ and $\bar{\varepsilon}$ (Str 4 $\left.\Delta_{f i x}, \bar{\varepsilon}\right)$, and with the Strategy 5 and different values for $\bar{\varepsilon}(\operatorname{Str} 5-\bar{\varepsilon})$. Strategies 2 and 3 will be tested when solving the integer problem in the complete Branch-and-Price framework (see Chapter 5).

We can notice that, for the first three classes of instances, stabilisation strategies 1 and 4 perform very differently depending on the choice of the parameter $\Delta$, whilst the choice of parameter $\bar{\varepsilon}$ is not very influential. A good choice of $\Delta$ leads to large improvements in the column generation algorithm, whilst bad choices significantly deteriorate the performance, such that a good tuning of the parameter is necessary. For A1 instances only, performances are similar between all tested strategies, and also similar to not using any stabilisation. Results for the best strategy for each instance size are represented in bold in Tables 4.7, 4.8, 4.9 and 4.10. We choose to define the best parameter configurations as the ones for which results are the best or the most constant (if not the best, very close to the best) for all instances sizes. For complete and partial graph instances the best strategies are Strategy 1 with $\Delta_{\text {init }}=0.5$ and $\bar{\varepsilon}=0.05$, Strategy 4 with $\Delta_{f i x}=0.5$ and $\bar{\varepsilon}=0.05$ and Strategy 5 with $\bar{\varepsilon}=0.05$. For Shioda et al.'s instances the best strategies are Strategy 1 with $\Delta_{\text {init }}=0.3$ and $\bar{\varepsilon}=0.01$, Strategy 4 with $\Delta_{\text {fix }}=0.3$ and $\bar{\varepsilon}=0.01$ and Strategy 5 with $\bar{\varepsilon}=0.01$. As we already mentioned, for A1 instances there are no predominant strategies, we choose Strategy 1 with $\Delta_{\text {init }}=0.5$ and $\bar{\varepsilon}=0.05$, Strategy 4 with $\Delta_{f i x}=0.5$ and $\bar{\varepsilon}=0.05$ and Strategy 5 with $\bar{\varepsilon}=0.05$ as they perform slightly better.

In Figure 4.10 we report the performance profile graphs for the solution time of these best strategies. We compare solving (HPDW-LR) with the column generation algorithm without any stabilisation to using Strategies 1 and 4 with the best parameters configuration from Tables 4.7, 4.8, 4.9 and 4.9 and to using Strategy 5 where only $\bar{\varepsilon}$ parameter is required. We can see Strategy 4 performs slightly better, and that for all classes of instances Strategy 5 performs similarly to the best choices for Strategy 4, with the advantage of no tuning of parameter $\Delta$ required. Furthermore, the best choice for parameter $\Delta$ differs between each class of instances, such that a separate tuning is required.

Using the best stabilisation strategies allows us to reduce the solution time of (HPDWLR) by between $35 \%$ and $60 \%$, and the number of generated columns by around $20-$ $30 \%$. The number of iterations of the column generation algorithm is also reduced by around $20-30 \%$, but we omitted detailed results to avoid even bigger tables. Bigger improvements occur in bigger instances, exactly where we would like to reduce the time and the number of columns.

In Figure 4.11 we plot the evolution of the primal and dual bound while solving one instance with the column generation algorithm, without any stabilisation and using the best three strategies. Also from these graphs we notice that Strategy 5 performs 


\begin{tabular}{|c|c|c|c|c|c|c|c|c|c|c|}
\hline \multirow{2}{*}{$\begin{array}{l}\text { Complete } \\
\text { graph }\end{array}$} & & \multicolumn{3}{|c|}{20 commodities } & \multicolumn{3}{|c|}{56 commodities } & \multicolumn{3}{|c|}{90 commodities } \\
\hline & & $20 \mathrm{a}$ & $56 \mathrm{a}$ & $90 \mathrm{a}$ & $20 \mathrm{a}$ & $56 \mathrm{a}$ & $90 \mathrm{a}$ & $20 \mathrm{a}$ & $56 \mathrm{a}$ & $90 \mathrm{a}$ \\
\hline \multirow[t]{2}{*}{ no stab } & Time & 0.199 & 0.726 & 1.57 & 4.13 & 12.7 & 22.3 & 25.84 & 62.26 & 108.99 \\
\hline & Cols & 335 & 655 & 924 & 1141 & 1765 & 2227 & 2103 & 3034 & 3749 \\
\hline Str 1 & Time & 0.269 & 1.887 & 5.528 & 3.287 & 17.20 & 40.83 & 16.24 & 58.82 & 135.42 \\
\hline $0.1,0.05$ & Cols & 431 & 1270 & 2192 & 990 & 1960 & 2968 & 1680 & 2697 & 3862 \\
\hline Str 1 & Time & 0.26 & 1.884 & 5.557 & 3.247 & 17.25 & 41.07 & 16.12 & 59.22 & 136.19 \\
\hline $0.1,0.01$ & Cols & 449 & 1299 & 2235 & 995 & 2012 & 3064 & 1669 & 2729 & 3925 \\
\hline Str 1 & Time & 0.21 & 1.182 & 3.169 & 2.945 & 11.81 & 25.18 & 15.02 & 43.38 & 88.22 \\
\hline $0.2,0.05$ & Cols & 385 & 938 & 1553 & 944 & 1636 & 2299 & 1634 & 2375 & 3159 \\
\hline Str 1 & Time & 0.211 & 1.202 & 3.197 & 2.919 & 11.87 & 25.41 & 14.90 & 43.18 & 88.44 \\
\hline $0.2,0.01$ & Cols & 403 & 979 & 1591 & 947 & 1682 & 2397 & 1628 & 2405 & 3234 \\
\hline Str 1 & Time & 0.175 & 0.926 & 2.311 & 2.753 & 9.833 & 20.02 & 14.62 & 39.02 & 74.43 \\
\hline $0.3,0.05$ & Cols & 342 & 806 & 1259 & 917 & 1497 & 2038 & 1622 & 2290 & 2943 \\
\hline Str 1 & Time & 0.179 & 0.964 & 2.363 & 2.8 & 9.913 & 20.13 & 14.34 & 38.96 & 74.51 \\
\hline $0.3,0.01$ & Cols & 361 & 852 & 1310 & 927 & 1557 & 2121 & 1600 & 2314 & 3003 \\
\hline Str 1 & Time & 0.156 & 0.723 & 1.651 & 2.794 & 9.003 & 17.35 & 14.70 & 38.29 & 72.30 \\
\hline $0.5,0.05$ & Cols & 318 & 683 & 988 & 924 & 1445 & 1915 & 1615 & 2282 & 2966 \\
\hline Str 1 & Time & 0.162 & 0.744 & 1.675 & 2.779 & 9.073 & 17.70 & 14.27 & 38.13 & 72.71 \\
\hline $0.5,0.01$ & Cols & 341 & 712 & 1024 & 930 & 1491 & 2005 & 1602 & 2304 & 3039 \\
\hline Str 4 & Time & 0.595 & 4.212 & 11.60 & 4.599 & 28.68 & 69.48 & 16.89 & 83.21 & 201.67 \\
\hline $0.1,0.05$ & Cols & 889 & 2657 & 4393 & 1263 & 3257 & 5237 & 1689 & 3713 & 5839 \\
\hline Str 4 & Time & 0.733 & 5.223 & 14.06 & 5.093 & 34.27 & 83.75 & 16.61 & 89.24 & 218.97 \\
\hline $0.1,0.01$ & Cols & 1123 & 3272 & 5277 & 1388 & 3894 & 6424 & 1713 & 4229 & 6810 \\
\hline Str 4 & Time & 0.283 & 1.715 & 4.564 & 2.809 & 13.57 & 30.56 & 11.91 & 43.29 & 95.82 \\
\hline $0.2,0.05$ & Cols & 506 & 1384 & 2262 & 919 & 1933 & 2906 & 1410 & 2431 & 3534 \\
\hline Str 4 & Time & 0.328 & 2.036 & 5.361 & 2.752 & 14.31 & 33.56 & 10.98 & 43.83 & 100.81 \\
\hline $0.2,0.01$ & Cols & 614 & 1676 & 2687 & 943 & 2168 & 3440 & 1351 & 2604 & 3956 \\
\hline Str 4 & Time & 0.194 & 1.125 & 2.793 & 2.496 & 10.11 & 21.33 & 11.35 & 36.28 & 74.47 \\
\hline $0.3,0.05$ & Cols & 388 & 1001 & 1555 & 854 & 1577 & 2224 & 1391 & 2171 & 3001 \\
\hline Str 4 & Time & 0.226 & 1.277 & 3.236 & 2.391 & 10.60 & 22.57 & 10.10 & 35.84 & 75.30 \\
\hline $0.3,0.01$ & Cols & 460 & 1169 & 1833 & 847 & 1739 & 2519 & 1294 & 2240 & 3182 \\
\hline Str 4 & Time & 0.163 & 0.731 & 1.739 & 2.516 & 8.58 & 17.44 & 12.52 & 35.22 & 68.72 \\
\hline $0.5,0.05$ & Cols & 324 & 692 & 1050 & 856 & 1405 & 1910 & 1464 & 2140 & 2822 \\
\hline Str 4 & Time & 0.175 & 0.829 & 1.914 & 2.431 & 8.675 & 17.93 & 11.38 & 35.07 & 67.92 \\
\hline $0.5,0.01$ & Cols & 363 & 803 & 1191 & 844 & 1491 & 2064 & 1378 & 2163 & 2934 \\
\hline Str 5 & Time & 0.194 & 0.632 & 1.192 & 2.526 & 8.199 & 16.52 & 11.90 & 35.63 & 71.29 \\
\hline 0.05 & Cols & 329 & 690 & 1003 & 888 & 1448 & 1977 & 1479 & 2208 & 2945 \\
\hline Str 5 & Time & 0.149 & 0.562 & 1.257 & 2.401 & 8.381 & 17.08 & 11.17 & 35.74 & 71.16 \\
\hline 0.01 & Cols & 366 & 756 & 1090 & 921 & 1572 & 2178 & 1431 & 2311 & 3106 \\
\hline
\end{tabular}

Table 4.7: Numerical results on different stabilisation strategies for (HPDW-LR) (best results in bold) - Complete graph instances

similarly to Strategy 4 with the best choice of parameter $\Delta$. Strategy 1 is less well performing. Furthermore the oscillation behaviour of the dual bound and the tailingoff effect that we noticed in Figure 4.1 are reduced a lot by the stabilisation, for both the complete graph instance (Figure 4.11(a)) and the Shioda et al.'s instance (Figure 4.11(b)). 


\begin{tabular}{|c|c|c|c|c|c|c|c|c|c|c|}
\hline \multirow{2}{*}{$\begin{array}{l}\text { Partial } \\
\text { graph }\end{array}$} & & \multicolumn{3}{|c|}{20 commodities } & \multicolumn{3}{|c|}{56 commodities } & \multicolumn{3}{|c|}{90 commodities } \\
\hline & & $20 \mathrm{a}$ & $56 \mathrm{a}$ & $90 \mathrm{a}$ & $20 \mathrm{a}$ & $56 \mathrm{a}$ & $90 \mathrm{a}$ & $20 \mathrm{a}$ & $56 a$ & $90 \mathrm{a}$ \\
\hline no stab & Time & 0.171 & 0.687 & 1.443 & 3.026 & 10.99 & 21.23 & 18.54 & 55.71 & 102.87 \\
\hline & Cols & 301 & 625 & 891 & 974 & 1699 & 2199 & 1853 & 2926 & 3747 \\
\hline Str 1 & Time & 0.208 & 1.537 & 4.341 & 2.297 & 12.5 & 30.92 & 11.11 & 42.55 & 95.79 \\
\hline $0.1,0.05$ & Cols & 390 & 1184 & 2055 & 829 & 1774 & 2722 & 1412 & 2377 & 3415 \\
\hline Str 1 & Time & 0.205 & 1.565 & 4.419 & 2.293 & 12.44 & 31.17 & 11.12 & 42.29 & 95.97 \\
\hline $0.1,0.01$ & Cols & 415 & 1237 & 2116 & 840 & 1824 & 2824 & 1419 & 2408 & 3478 \\
\hline Str 1 & Time & 0.183 & 1.054 & 2.63 & 2.056 & 8.748 & 20.09 & 10.12 & 33.13 & 66.88 \\
\hline $0.2,0.05$ & Cols & 348 & 921 & 1490 & 777 & 1465 & 2113 & 1352 & 2117 & 2839 \\
\hline Str 1 & Time & 0.179 & 1.095 & 2.703 & 2.086 & 8.822 & 20.27 & 10.03 & 33.17 & 67.08 \\
\hline $0.2,0.01$ & Cols & 367 & 966 & 1536 & 786 & 1512 & 2194 & 1354 & 2152 & 2890 \\
\hline Str 1 & Time & 0.162 & 0.866 & 2.052 & 1.975 & 7.642 & 16.47 & 9.802 & 30.05 & 58.83 \\
\hline $0.3,0.05$ & Cols & 323 & 798 & 1246 & 756 & 1359 & 1891 & 1344 & 2014 & 2629 \\
\hline Str 1 & Time & 0.162 & 0.891 & 2.093 & 1.987 & 7.682 & 16.63 & 9.626 & 30.14 & 59.01 \\
\hline $0.3,0.01$ & Cols & 342 & 838 & 1281 & 771 & 1404 & 1965 & 1336 & 2053 & 2702 \\
\hline Str 1 & Time & 0.147 & 0.695 & 1.569 & 1.968 & 7.19 & 14.744 & 10.21 & 30.12 & 58.10 \\
\hline $0.5,0.05$ & Cols & 306 & 685 & 1010 & 763 & 1332 & 1775 & 1369 & 2045 & 2648 \\
\hline Str 1 & Time & 0.153 & 0.727 & 1.606 & 1.954 & 7.251 & 15.02 & 10.16 & 30.09 & 58.68 \\
\hline $0.5,0.01$ & Cols & 328 & 726 & 1057 & 773 & 1378 & 1859 & 1370 & 2082 & 2713 \\
\hline Str 4 & Time & 0.518 & 3.978 & 10.57 & 3.17 & 21.93 & 56.79 & 11.22 & 62.01 & 153.45 \\
\hline $0.1,0.05$ & Cols & 851 & 2726 & 4461 & 1108 & 3103 & 5134 & 1449 & 3443 & 55.37 \\
\hline Str 4 & Time & 0.694 & 5.171 & 13.34 & 3.72 & 27.54 & 71.67 & 11.52 & 68.78 & 171.44 \\
\hline $0.1,0.01$ & Cols & 1123 & 3483 & 5569 & 1303 & 3833 & 6390 & 1544 & 4078 & 6679 \\
\hline Str 4 & Time & 0.232 & 1.654 & 4.127 & 2.007 & 10.61 & 25.69 & 7.977 & 33.99 & 74.83 \\
\hline $0.2,0.05$ & Cols & 483 & 1431 & 2292 & 781 & 1807 & 2816 & 1183 & 2234 & 3303 \\
\hline Str 4 & Time & 0.307 & 2.078 & 5.027 & 2.084 & 11.84 & 28.51 & 7.708 & 35.74 & 79.56 \\
\hline $0.2,0.01$ & Cols & 615 & 1782 & 2805 & 844 & 2144 & 3396 & 1182 & 2488 & 3777 \\
\hline Str 4 & Time & 0.181 & 1.067 & 2.539 & 1.739 & 7.835 & 18.04 & 7.86 & 28.42 & 58.82 \\
\hline $0.3,0.05$ & Cols & 375 & 1011 & 1592 & 705 & 1448 & 2126 & 1171 & 1958 & 2716 \\
\hline Str 4 & Time & 0.209 & 1.25 & 3.027 & 1.759 & 8.48 & 19.54 & 7.284 & 29.17 & 61.32 \\
\hline $0.3,0.01$ & Cols & 455 & 1229 & 1929 & 735 & 1635 & 2477 & 1132 & 2081 & 3008 \\
\hline Str 4 & Time & 0.145 & 0.738 & 1.663 & 1.792 & 6.993 & 14.77 & 8.888 & 28.41 & 56.43 \\
\hline $0.5,0.05$ & Cols & 308 & 726 & 1080 & 715 & 1313 & 1794 & 1251 & 1957 & 2607 \\
\hline Str 4 & Time & 0.159 & 0.812 & 1.873 & 1.761 & 7.255 & 15.57 & 8.249 & 28.21 & 57.21 \\
\hline $0.5,0.01$ & Cols & 351 & 833 & 1254 & 725 & 1410 & 1989 & 1200 & 2016 & 2758 \\
\hline Str 5 & Time & 0.16 & 0.604 & 1.156 & 1.859 & 7.158 & 14.51 & 8.318 & 29.92 & 58.15 \\
\hline 0.05 & Cols & 314 & 679 & 1025 & 757 & 1413 & 1903 & 1236 & 2066 & 2736 \\
\hline Str 5 & Time & 0.123 & 0.541 & 1.195 & 1.805 & 7.277 & 15.14 & 7.993 & 30.13 & 59.53 \\
\hline 0.01 & Cols & 353 & 752 & 1108 & 809 & 1535 & 2085 & 1243 & 2182 & 2926 \\
\hline
\end{tabular}

Table 4.8: Numerical results on different stabilisation strategies for (HPDW-LR) (best results in bold) - Partial graph instances 


\begin{tabular}{|c|c|c|c|c|c|c|c|c|c|c|}
\hline \multirow{2}{*}{\multicolumn{2}{|c|}{$\begin{array}{l}\text { Shioda et al. } \\
\text { instances }\end{array}$}} & \multicolumn{3}{|c|}{40 commodities } & \multicolumn{3}{|c|}{60 commodities } & \multicolumn{3}{|c|}{80 commodities } \\
\hline & & $20 \mathrm{a}$ & $40 \mathrm{a}$ & $60 \mathrm{a}$ & $20 \mathrm{a}$ & $40 \mathrm{a}$ & $60 \mathrm{a}$ & $20 \mathrm{a}$ & $40 \mathrm{a}$ & $60 \mathrm{a}$ \\
\hline \multirow[t]{2}{*}{ no stab } & Time & 5.51 & 7.79 & 11.39 & 43.12 & 47.04 & 56.83 & 198.38 & 221.28 & 260.09 \\
\hline & Cols & 1413 & 1714 & 2015 & 2721 & 3046 & 3506 & 4586 & 5130 & 5645 \\
\hline Str 1 & Time & 3.48 & 7.61 & 15.27 & 19.29 & 28.58 & 50.38 & 88.13 & 92.78 & 137.85 \\
\hline $0.1,0.05$ & Cols & 1122 & 1566 & 2175 & 1976 & 2377 & 2952 & 3251 & 3513 & 4104 \\
\hline Str 1 & Time & 3.54 & 7.61 & 15.25 & 19.27 & 28.31 & 51.66 & 89.7 & 91.17 & 150.78 \\
\hline $0.1,0.01$ & Cols & 1154 & 1608 & 2211 & 2000 & 2431 & 3048 & 3278 & 3583 & 4269 \\
\hline Str 1 & Time & 3.2 & 5.3 & 9.45 & 17.23 & 23.15 & 32.31 & 82.92 & 75.27 & 113.4 \\
\hline $0.2,0.05$ & Cols & 1095 & 1351 & 1699 & 1892 & 2184 & 2536 & 3200 & 3303 & 3706 \\
\hline Str 1 & Time & 3.22 & 5.34 & 9.61 & 17.07 & 21.26 & 38.81 & 87.45 & 84.54 & 124.26 \\
\hline $0.2,0.01$ & Cols & 1130 & 1386 & 1769 & 1923 & 2165 & 2710 & 3299 & 3476 & 3967 \\
\hline Str 1 & Time & 3.16 & 4.58 & 7.67 & 17.89 & 19.33 & 32.43 & 87.06 & 68.11 & 121.72 \\
\hline $0.3,0.05$ & Cols & 1079 & 1285 & 1586 & 1918 & 2071 & 2547 & 3234 & 3204 & 3844 \\
\hline Str 1 & Time & 3.06 & 4.59 & 7.69 & 17.43 & 19.78 & 30.26 & 81.52 & 71.68 & 103.64 \\
\hline $0.3,0.01$ & Cols & 1097 & 1333 & 1639 & 1934 & 2144 & 2572 & 3153 & 3349 & 3821 \\
\hline Str 1 & Time & 2.85 & 4.54 & 7.05 & 18.53 & 20.32 & 30.73 & 94.35 & 93 & 132.46 \\
\hline $0.5,0.05$ & Cols & 1040 & 1297 & 1558 & 1963 & 2155 & 2520 & 3315 & 3576 & 4078 \\
\hline Str 1 & Time & 2.91 & 4.55 & 7.1 & 18.42 & 20.45 & 30.07 & 108.07 & 87.23 & 102.49 \\
\hline $0.5,0.01$ & Cols & 1061 & 1342 & 1608 & 1978 & 2215 & 2574 & 3436 & 3567 & 3941 \\
\hline Str 4 & Time & 5.21 & 12.72 & 28.9 & 22.72 & 39.67 & 72.01 & 93.12 & 116.57 & 252.32 \\
\hline $0.1,0.05$ & Cols & 1468 & 2432 & 3540 & 2128 & 3076 & 4239 & 3150 & 4032 & 5436 \\
\hline Str 4 & Time & 4.79 & 13.44 & 26.47 & 18.51 & 37.42 & 71.26 & 57.34 & 118.51 & 187.9 \\
\hline $0.1,0.01$ & Cols & 1530 & 2758 & 3983 & 2017 & 3214 & 4622 & 2673 & 4108 & 5474 \\
\hline Str 4 & Time & 3.24 & 5.98 & 10.96 & 15.53 & 23.37 & 33.78 & 68.38 & 71.19 & 131.49 \\
\hline $0.2,0.05$ & Cols & 1109 & 1535 & 2053 & 1809 & 2251 & 2731 & 2880 & 3252 & 3850 \\
\hline Str 4 & Time & 2.82 & 6.03 & 11.34 & 10.97 & 18.63 & 34.08 & 48.84 & 65.15 & 97.72 \\
\hline $0.2,0.01$ & Cols & 1092 & 1657 & 2250 & 1550 & 2144 & 2857 & 2393 & 2978 & 3575 \\
\hline Str 4 & Time & 2.72 & 4.57 & 7.81 & 15.43 & 17.91 & 27.4 & 71.9 & 70.42 & 81.62 \\
\hline $0.3,0.05$ & Cols & 1016 & 1311 & 1649 & 1758 & 2000 & 2387 & 2897 & 3161 & 3400 \\
\hline Str 4 & Time & 2.3 & 4.43 & 7.97 & 11.2 & 16.57 & 25.4 & 50.04 & 58.44 & 89.59 \\
\hline $0.3,0.01$ & Cols & 968 & 1347 & 1737 & 1547 & 1928 & 2384 & 2395 & 2730 & 3323 \\
\hline Str 4 & Time & 2.62 & 4.24 & 6.38 & 14.93 & 20.79 & 29.89 & 90.16 & 77.53 & 88.56 \\
\hline $0.5,0.05$ & Cols & 985 & 1249 & 1456 & 1732 & 2043 & 2393 & 2999 & 3149 & 3488 \\
\hline Str 4 & Time & 2.35 & 4.14 & 6.44 & 12.86 & 18.06 & 27.31 & 62.32 & 74.1 & 114.09 \\
\hline $0.5,0.01$ & Cols & 956 & 1254 & 1495 & 1616 & 1595 & 2338 & 2555 & 3002 & 3545 \\
\hline Str 5 & Time & 2.95 & 4.18 & 6.16 & 16.48 & 21.42 & 26.08 & 70.6 & 68.96 & 76.77 \\
\hline 0.05 & Cols & 1068 & 1299 & 1543 & 1862 & 2174 & 2438 & 2972 & 3215 & 3470 \\
\hline Str 5 & Time & 2.6 & 3.98 & 6.18 & 14.61 & 17.06 & 24.49 & 69.29 & 66.9 & 81.02 \\
\hline 0.01 & Cols & 1056 & 1340 & 1585 & 1773 & 2018 & 2444 & 2910 & 3114 & 3453 \\
\hline
\end{tabular}

Table 4.9: Numerical results on different stabilisation strategies for (HPDW-LR) (best results in bold) - Shioda et al. instances 


\begin{tabular}{|c|c|c|c|c|c|c|c|c|c|c|}
\hline \multirow{2}{*}{$\begin{array}{l}\text { A1 } \\
\text { instances }\end{array}$} & & \multicolumn{3}{|c|}{21 commodities } & \multicolumn{3}{|c|}{55 commodities } & \multicolumn{3}{|c|}{91 commodities } \\
\hline & & $21 \mathrm{a}$ & $55 \mathrm{a}$ & $91 \mathrm{a}$ & $21 \mathrm{a}$ & $55 \mathrm{a}$ & $91 \mathrm{a}$ & $21 \mathrm{a}$ & $55 \mathrm{a}$ & $91 \mathrm{a}$ \\
\hline \multirow[t]{2}{*}{ no stab } & Time & 0.07 & 0.22 & 0.46 & 0.48 & 1.69 & 3.85 & 3.14 & 8.63 & 16.45 \\
\hline & Cols & 137 & 333 & 460 & 347 & 572 & 863 & 727 & 1185 & 1527 \\
\hline Str 1 & Time & 0.06 & 0.26 & 0.51 & 0.46 & 1.70 & 3.95 & 2.72 & 7.99 & 16.23 \\
\hline $0.1,0.05$ & Cols & 158 & 396 & 521 & 344 & 631 & 959 & 681 & 1194 & 1617 \\
\hline Str 1 & Time & 0.05 & 0.25 & 0.52 & 0.47 & 1.73 & 4.02 & 2.63 & 7.95 & 16.37 \\
\hline $0.1,0.01$ & Cols & 165 & 410 & 531 & 368 & 659 & 1005 & 690 & 1230 & 1684 \\
\hline Str 1 & Time & 0.05 & 0.24 & 0.46 & 0.49 & 1.66 & 3.90 & 2.59 & 7.87 & 16.08 \\
\hline $0.2,0.05$ & Cols & 158 & 375 & 482 & 351 & 609 & 946 & 674 & 1176 & 1586 \\
\hline Str 1 & Time & 0.05 & 0.27 & 0.47 & 0.48 & 1.68 & 3.92 & 2.64 & 7.92 & 16.01 \\
\hline $0.2,0.01$ & Cols & 143 & 338 & 487 & 347 & 585 & 878 & 691 & 1187 & 1547 \\
\hline Str 1 & Time & 0.05 & 0.23 & 0.47 & 0.47 & 1.63 & 3.82 & 2.80 & 7.71 & 15.55 \\
\hline $0.3,0.05$ & Cols & 160 & 356 & 486 & 342 & 607 & 923 & 695 & 1154 & 1531 \\
\hline Str 1 & Time & 0.05 & 0.23 & 0.49 & 0.46 & 1.67 & 3.88 & 2.95 & 7.82 & 15.42 \\
\hline $0.3,0.01$ & Cols & 167 & 367 & 501 & 356 & 634 & 961 & 707 & 1197 & 1586 \\
\hline Str 1 & Time & 0.04 & 0.22 & 0.48 & 0.49 & 1.63 & 3.64 & 2.87 & 8.29 & 15.79 \\
\hline $0.5,0.05$ & Cols & 143 & 338 & 487 & 347 & 585 & 878 & 691 & 1187 & 1547 \\
\hline Str 1 & Time & 0.05 & 0.22 & 0.49 & 0.46 & 1.69 & 3.74 & 2.77 & 8.07 & 16.11 \\
\hline $0.5,0.01$ & Cols & 151 & 346 & 496 & 356 & 615 & 917 & 702 & 1220 & 1603 \\
\hline Str 4 & Time & 0.07 & 0.50 & 1.00 & 0.71 & 3.09 & 7.66 & 3.08 & 11.37 & 26.39 \\
\hline $0.1,0.05$ & Cols & 229 & 722 & 945 & 531 & 1178 & 1863 & 879 & 1853 & 2792 \\
\hline Str 4 & Time & 0.08 & 0.52 & 1.05 & 0.76 & 3.44 & 9.02 & 3.19 & 13.24 & 30.54 \\
\hline $0.1,0.01$ & Cols & 238 & 739 & 969 & 598 & 1317 & 2177 & 1008 & 2274 & 3375 \\
\hline Str 4 & Time & 0.06 & 0.40 & 0.70 & 0.52 & 2.07 & 5.02 & 2.68 & 8.36 & 18.14 \\
\hline $0.2,0.05$ & Cols & 203 & 574 & 690 & 413 & 782 & 1244 & 725 & 1305 & 1879 \\
\hline Str 4 & Time & 0.07 & 0.43 & 0.78 & 0.56 & 2.38 & 5.80 & 2.57 & 8.81 & 19.39 \\
\hline $0.2,0.01$ & Cols & 158 & 385 & 485 & 348 & 588 & 886 & 626 & 1083 & 1485 \\
\hline Str 4 & Time & 0.06 & 0.31 & 0.56 & 0.47 & 1.82 & 4.14 & 2.46 & 7.66 & 15.45 \\
\hline $0.3,0.05$ & Cols & 182 & 474 & 562 & 372 & 686 & 1029 & 660 & 1153 & 1606 \\
\hline Str 4 & Time & 0.06 & 0.38 & 0.63 & 0.49 & 1.94 & 4.62 & 2.29 & 7.71 & 16.28 \\
\hline $0.3,0.01$ & Cols & 196 & 513 & 611 & 411 & 768 & 1171 & 671 & 1288 & 1776 \\
\hline Str 4 & Time & 0.05 & 0.25 & 0.49 & 0.48 & 1.61 & 3.64 & 2.40 & 7.21 & 14.62 \\
\hline $0.5,0.05$ & Cols & 158 & 385 & 485 & 348 & 588 & 886 & 626 & 1083 & 1485 \\
\hline Str 4 & Time & 0.05 & 0.27 & 0.52 & 0.45 & 1.70 & 3.71 & 2.22 & 7.31 & 14.92 \\
\hline $0.5,0.01$ & Cols & 172 & 409 & 516 & 368 & 656 & 957 & 643 & 1187 & 1590 \\
\hline Str 5 & Time & 0.04 & 0.25 & 0.47 & 0.46 & 1.63 & 3.62 & 2.50 & 7.11 & 15.15 \\
\hline 0.05 & Cols & 145 & 354 & 478 & 345 & 585 & 866 & 637 & 1090 & 1500 \\
\hline Str 5 & Time & 0.05 & 0.26 & 0.49 & 0.47 & 1.70 & 3.66 & 2.32 & 7.40 & 15.13 \\
\hline 0.01 & Cols & 157 & 370 & 500 & 368 & 645 & 935 & 677 & 1213 & 1614 \\
\hline
\end{tabular}

Table 4.10: Numerical results on different stabilisation strategies for (HPDW-LR) (best results in bold) - A1 instances 


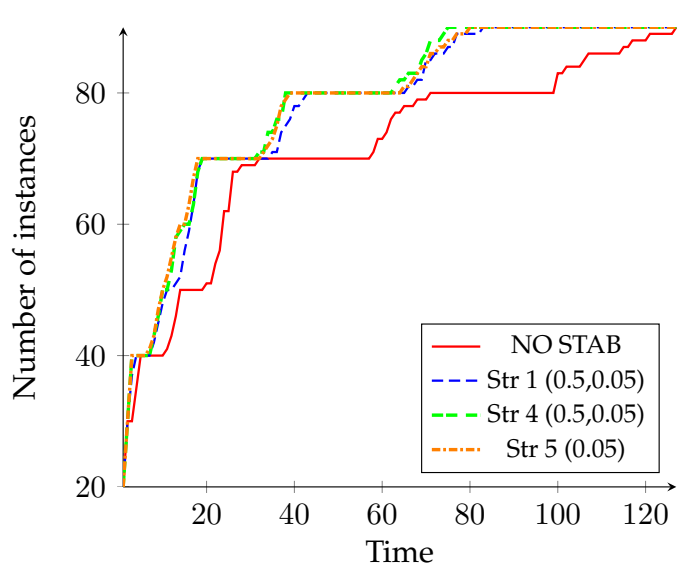

(a) Complete graph instances

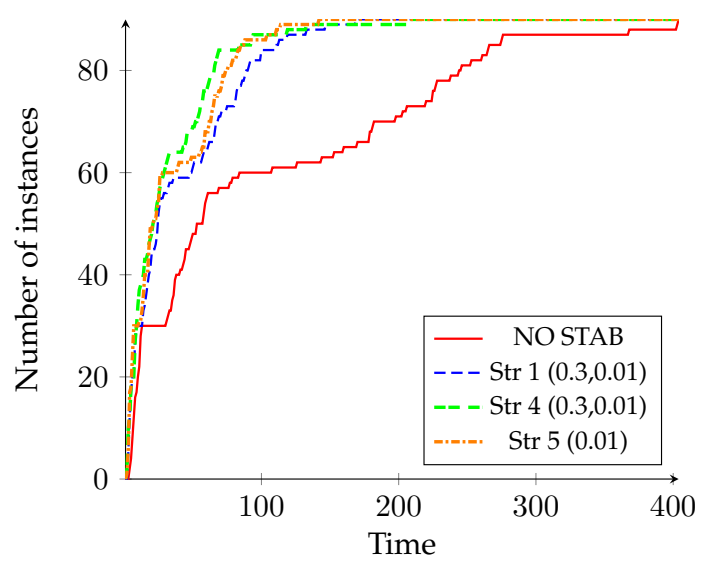

(c) Shioda et al.'s instances

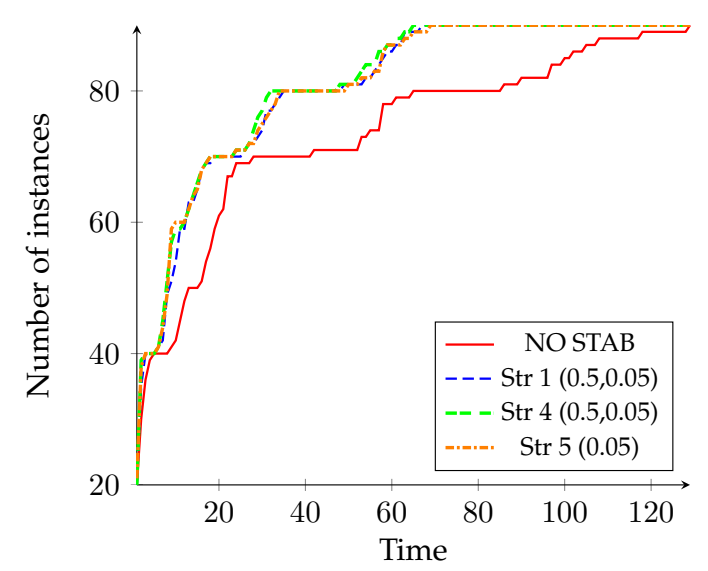

(b) Partial graph instances

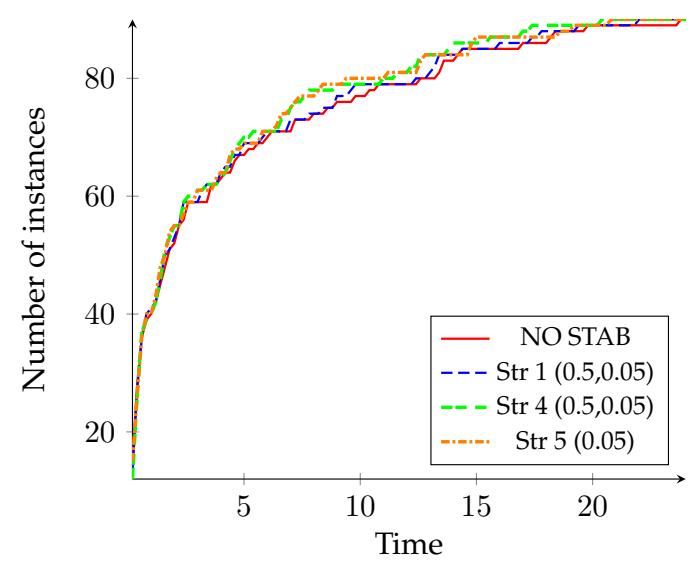

(d) A1 instances

Figure 4.10: Performance profile graphs on different stabilisation strategies for (HPDW-LR)

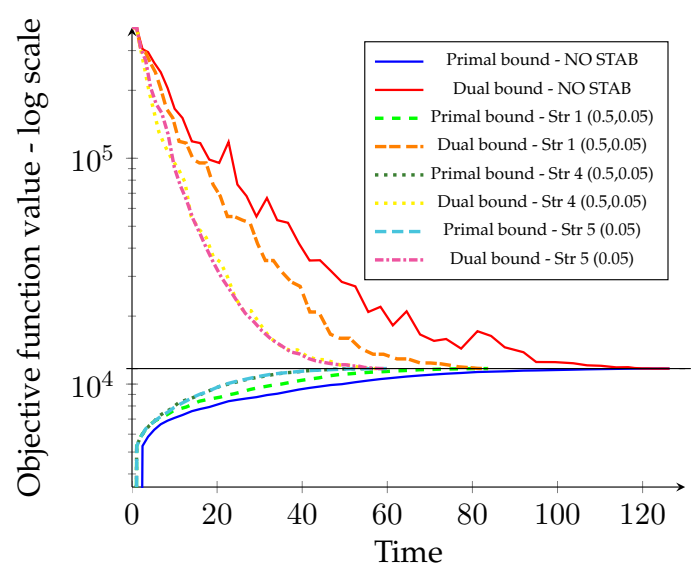

(a) 90k 90a complete graph instance

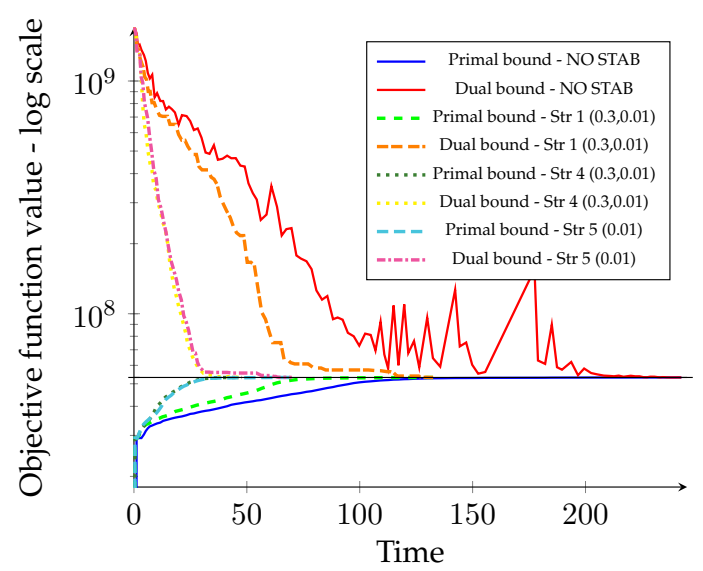

(b) $80 \mathrm{k} 60 \mathrm{a}$ Shioda et al. instance

Figure 4.11: Primal and dual bounds evolution while solving (HPDW) with column generation - Different stabilisation strategies performance 


\subsection{Best configuration of the column generation algorithm for the linear relaxation of the NPP with connected toll arcs}

In this chapter we introduced a Dantzig-Wolfe reformulation for the NPP with connected toll arcs (HPDW) and a column generation algorithm to solve its linear relaxation. Various schemes have been proposed to improve the algorithm, and it is possible to combine all the proposed strategies leading to many different configurations of the column generation algorithm. We therefore tested them to choose the best configuration for our algorithm applied to our instances' set.

\subsubsection{List of parameters to define the column generation algorithm}

We associate a parameter to each feature that can be modified in the column generation algorithm:

-c strategy to initialise columns for the column generation algorithm (see Section 4.6.1). Possible values $\mathrm{c}=\{1,2\}$.

1: strategy INIT 0 (all null columns), 2: strategy INIT 1 (with heuristic).

-v maximum number of columns added at each iteration of column generation, for each path (see Section 4.7). Possible values $\mathrm{v}=\{1,2, \ldots,|\mathcal{K}|\}$.

-s stabilisation strategy (see Section 4.9.2). Possible values $\mathrm{s}=\{0,1,2,3,4,5\}$.

0: stabilisation disabled, 1: Strategy 1 (1-gap), 2: Strategy 2 (from Pessoa et al. 2010)), 3: Strategy 3 (mixed 1 and 2), 4: Strategy 4 (fixed), 5: Strategy 5 (autoadaptive with subgradient, from Pessoa et al. (2013)). As detailed in Section 4.9.3. for (HPDW-LR) Strategy 2 is equal to Strategy 4 and Strategy 3 to Strategy 1, so here we test only $s=\{0,1,4,5\}$.

Only if $s>0$ :

-e parameter $\bar{\varepsilon}$ to disable stabilisation when the gap is smaller than this epsilon. Possible values $0.00001<e<1$.

Only if $\mathrm{s}=\{1,4\}$ :

-d parameter $\Delta$ for the convex combination of dual values during stabilisation. Possible values $0.00001<d \leq 1$.

In SCIP framework, in which we implemented the column generation algorithm (see Achterberg, 2009), it is also possible to "remove" variables (i.e. columns) due to ageing. The age is calculated as the number of LP iterations in which a variable does not enter the basis. There is a difference between "remove" and "delete": removed variables still exist in the model, but are kept aside and tested at each iteration with a specific pricer for possible reinsertion, whilst deleted variables do not exist any more and all the associated values and structures should be deleted accordingly, making this operation 
much more complex from an implementation point of view. We therefore decided to consider only the removing option, and if it reveals useful, to add the deleting possibility in a second stage. We set the following parameters to include this possibility in our framework:

- $\mathbf{m}$ columns are removable due to ageing. Possible values $\mathbf{m}=\{0,1\}$.

0 : not removable, 1: removable.

Only for $\mathrm{m}=1$ :

-a age after which columns are removed. Possible values $\mathbf{a}=\{0,1,2,3, \ldots\}$.

Finally the algorithm used to solve the linear problems (i.e. the linear relaxation of the restricted (MP) for each iteration of the column generation algorithm) can be chosen, and we set the following parameters:

-S algorithm for solving initial LP relaxations. Possible values $S=\{0,1,2\}$.

0: automatic simplex, 1: primal simplex, 2: dual simplex.

- $\mathbf{R}$ algorithm for resolving LP relaxations if a starting basis exists. Possible values $\mathrm{R}=\{0,1,2\}$.

0: automatic simplex, 1: primal simplex, 2: dual simplex.

\subsubsection{Automatic tuning of the parameters}

The column generation algorithm is defined by nine different parameters, many with several possible values, leading to a big number of different configurations of the algorithm. In the previous sections we tested the performances of the possible values for each parameter separately, but combining them could lead to different results such that further testing is necessary. Due to the large number of possible configurations, manually testing them would require a big effort. Instead the parameters tuning has been performed by Leslie Pérez Cáceres from IRIDIA research group (ULB) using irace, which is a tool for the automatic configuration of algorithms developed by LópezIbánez et al. (2011), also from IRIDIA. Its main purpose is to automatically configure optimisation algorithms, by finding the best configuration of the parameters given a set of instances of the problem. Parameters' values define a configuration: they can be numerical or categorical (i.e. they take discrete values without an implicit order of their values), and it is also possible to specify conditional rules between parameters. A cost measure is used to assess the quality of a configuration over each instance of the set, which could be the best objective function value found within a given computational time, or the computational time required to solve the instance, possibly bounded by a maximum cut-off time. Then, irace searches for the configuration which optimises a function of the configurations cost over the whole set of instances, which is typically the expected cost value or mean cost. It implements an iterated racing framework to 
search through the configurations space, which works as follows. A race starts with a finite set of candidate configurations and is composed by several steps. At each step the candidate configurations are evaluated on each instance of the set, and after each step, statistical tests discard the candidates performing worse than the others. Then the race continues on the remaining candidate configurations, until the predefined minimum number of remaining configurations or the predefined computational budget are reached. Note that different tests can be used and that the predefined computational budget may be an overall computational time or a number of experiments. For more details about the exact implementation and on how to use it, we refer the interested reader to López-lbánez et al. (2011). We now report the results found by tuning our column generation algorithm with irace.

The tuning was first performed separately on each set of instances, and then considering all types together. As the time to solve the linear relaxation is relatively small we were able to use also the biggest instances. The results for each type of instances are reported in Table 4.11, whilst in Table 4.12 we report the results for the overall tuning. For each class of instances and the overall set irace was run five times, and we report the best configuration of parameters that was found in each run. In Figure 4.12 we report the performance profile graph for the time to solve (HPDW-LR) with the best configuration found by irace in each of the five runs, for the separate tuning on each class of instances. Then, in Figure 4.13, we compare, always with a performance profile graph, the best configuration out of the five runs of the separate tuning (noted as "best sep. R(number)") with the five best configurations found with the overall tuning on all instances.

\begin{tabular}{|c|c|c|c|c|c|c|c|c|c|c|c|c|c|c|c|c|c|c|c|}
\hline \multicolumn{10}{|c|}{ Complete graph instances } & \multicolumn{10}{|c|}{ Partial graph instances } \\
\hline Run & $-\mathrm{C}$ & $-\mathrm{V}$ & $-s$ & $-d$ & $-\mathrm{e}$ & $-\mathrm{m}$ & $-\mathrm{a}$ & $-S$ & $-\mathrm{R}$ & Run & $-\mathrm{c}$ & $-\mathrm{V}$ & $-s$ & $-d$ & $-\mathrm{e}$ & $-m$ & $-\mathrm{a}$ & $-S$ & $-\mathrm{R}$ \\
\hline 1 & 1 & 1 & 5 & - & 0.13 & 0 & - & 2 & 2 & 1 & 1 & 1 & 4 & 0.57 & 0.10 & 1 & 32 & 2 & 2 \\
\hline 2 & 2 & 1 & 4 & 0.60 & 0.22 & 0 & - & 0 & 2 & 2 & 1 & 1 & 4 & 0.68 & 0.59 & 0 & - & 2 & 2 \\
\hline 3 & 1 & 1 & 5 & - & 0.04 & 1 & 41 & 0 & 2 & 3 & 2 & 1 & 5 & - & 0.21 & 1 & 41 & 2 & 2 \\
\hline 4 & 2 & 1 & 5 & - & 0.14 & 0 & - & 2 & 2 & 4 & 1 & 1 & 5 & - & 0.03 & 1 & 35 & 2 & 2 \\
\hline 5 & 1 & 1 & 5 & - & 0.49 & 0 & - & 2 & 2 & 5 & 2 & 1 & 5 & - & 0.03 & 0 & - & 0 & 2 \\
\hline \multicolumn{10}{|c|}{ Shioda et al. instances } & \multicolumn{10}{|c|}{ A1 instances } \\
\hline Run & $-\mathrm{c}$ & $-\mathrm{v}$ & $-s$ & $-\mathrm{d}$ & $-\mathrm{e}$ & $-\mathrm{m}$ & $-\mathrm{a}$ & $-S$ & $-\mathrm{R}$ & Run & $-\mathrm{C}$ & $-\mathrm{V}$ & $-s$ & $-\mathrm{d}$ & $-\mathrm{e}$ & $-m$ & $-\mathrm{a}$ & $-S$ & $-\mathrm{R}$ \\
\hline 1 & 2 & 1 & 4 & 0.36 & 0.60 & 0 & - & 2 & 2 & 1 & 2 & 1 & 1 & 0.58 & 0.26 & 1 & 49 & 1 & 2 \\
\hline 2 & 2 & 1 & 4 & 0.32 & 0.01 & 1 & 48 & 2 & 2 & 2 & 2 & 1 & 5 & - & 0.35 & 0 & - & 1 & 2 \\
\hline 3 & 2 & 1 & 5 & - & 0.35 & 0 & - & 2 & 2 & 3 & 2 & 1 & 5 & - & 0.17 & 0 & - & 1 & 2 \\
\hline 4 & 1 & 1 & 4 & 0.21 & 0.31 & 0 & - & 0 & 2 & 4 & 2 & 1 & 4 & 0.56 & 0.72 & 0 & - & 0 & 2 \\
\hline 5 & 2 & 1 & 4 & 0.38 & 0.09 & 1 & 41 & 0 & 2 & 5 & 2 & 1 & 1 & 0.73 & 0.55 & 0 & - & 1 & 1 \\
\hline
\end{tabular}

Table 4.11: Best configurations of parameters for each instances type irace results 


\begin{tabular}{c|ccccccccc} 
Run & $-\mathrm{c}$ & $-\mathrm{v}$ & $-\mathrm{s}$ & $-\mathrm{d}$ & $-\mathrm{e}$ & $-\mathrm{m}$ & $-\mathrm{a}$ & $-\mathrm{S}$ & $-\mathrm{R}$ \\
\hline 1 & 2 & 1 & 5 & - & 0.06 & 0 & - & 1 & 2 \\
2 & 1 & 1 & 4 & 0.52 & 0.17 & 0 & - & 2 & 2 \\
3 & 2 & 1 & 5 & - & 0.45 & 0 & - & 0 & 2 \\
4 & 2 & 1 & 4 & 0.55 & 0.44 & 0 & - & 0 & 2 \\
5 & 2 & 1 & 1 & 0.65 & 0.02 & 0 & - & 2 & 2
\end{tabular}

Table 4.12: Best configurations of parameters for all instances types irace results

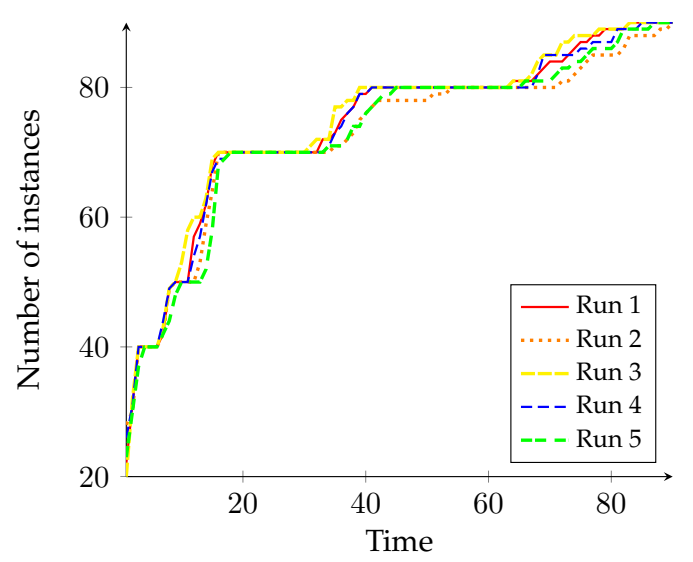

(a) Complete graph instances

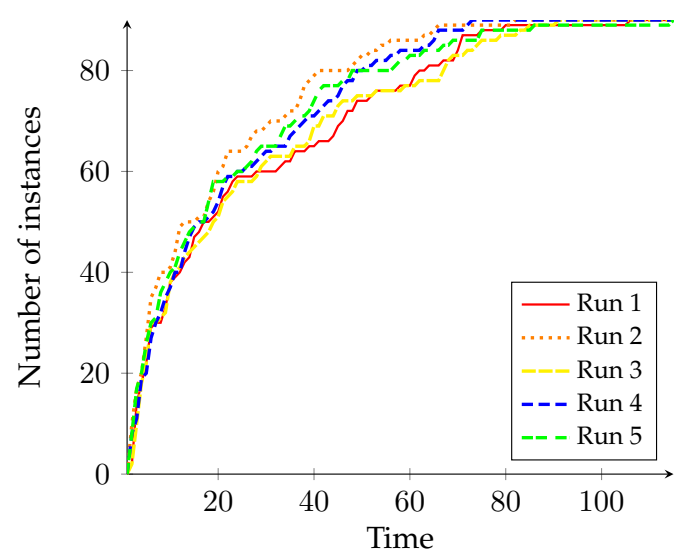

(c) Shioda et al.'s instances

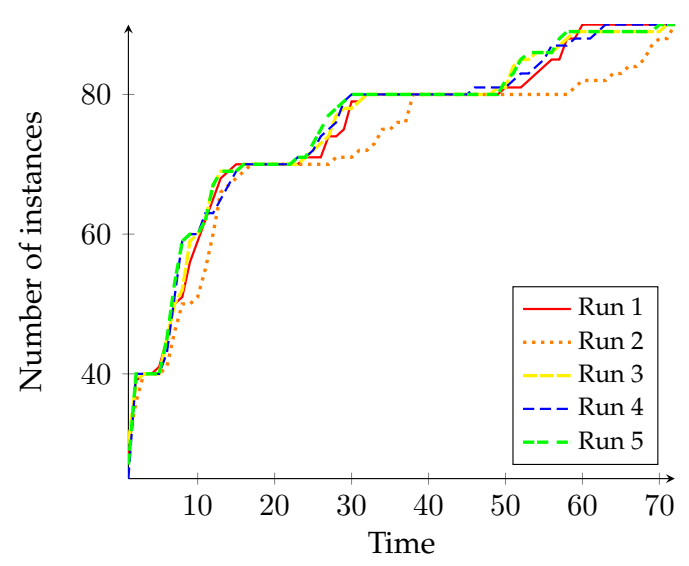

(b) Partial graph instances

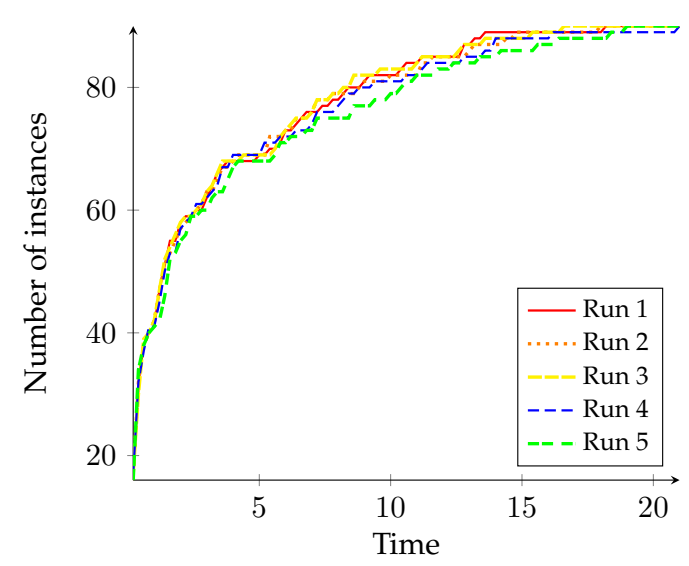

(d) A1 instances

Figure 4.12: Performance profile graphs on the best configurations of the column generation algorithm found by irace for each instance type

First of all from the graphs in Figures 4.12 and 4.13 we notice that the performances of the best configuration found by each run of the tuning are very similar, proving the stability of irace's results. Furthermore, when in Figure 4.13 we compare the best configuration found for one specific type of instances (the black dashed/dotted line on each graph) with the tuning results found for the mixed set of instances, we also notice that the performances are very similar, meaning that the best configuration of the algorithm does not depend much on the class of instances on which it is tuned, and 


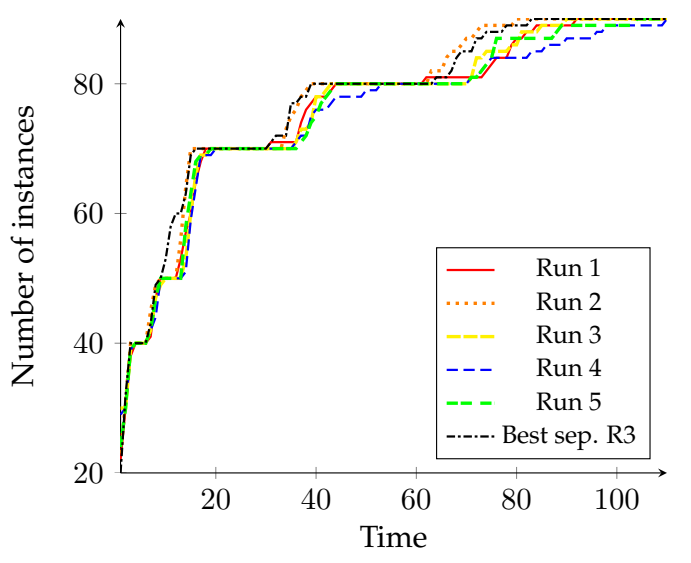

(a) Complete graph instances

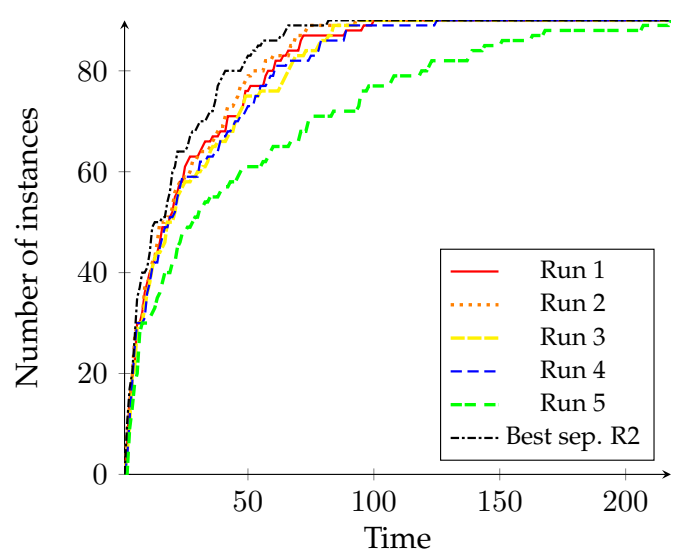

(c) Shioda et al.'s instances

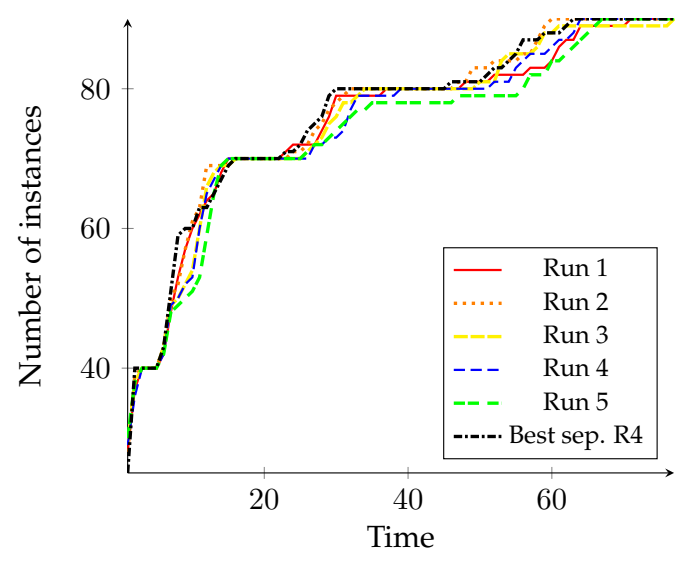

(b) Partial graph instances

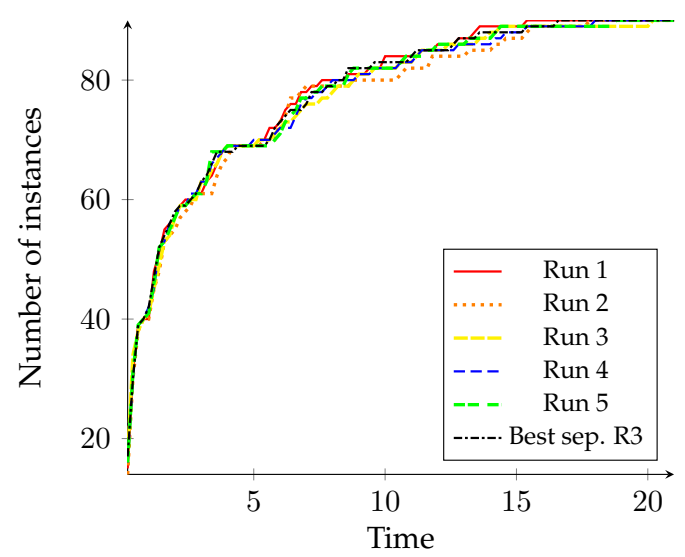

(d) A1 instances

Figure 4.13: Performance profile graphs on the best configurations of the column generation algorithm found by irace with the overall tuning (compared with the best configuration found in the separate tuning)

that the algorithm performance is quite stable for the good configurations. Only for Shioda et al. instances (see Figure 4.13(c)) the fifth best configuration produces worst results compared with the others, and the best configuration of the separate tuning (the second one) performs slightly better. Analysing the graphs of Figure 4.13 in details we can conclude that the first three configurations provided in Table 4.12 are the best for all class of instances.

We now analyse the configurations found, to discuss if the results of the tuning of the whole algorithm are in accordance to the results of testing each element separately that we presented in the previous sections.

The first parameter is "- $c$ " which refers to the initialisation strategy detailed in Section 4.6.1. The tuning shows that the second initialisation strategy is overall slightly better than the first one, which complies with the previous results. Even if for complete and partial graph instances the tuning finds good configurations with both strategies, they perform very similarly, apart from the second configuration found for the partial 
graph instances which is worst than the others and uses the first initialisation strategies. We therefore conclude that either the second initialisation is better or the is not much a difference between them, so we can use the second one without any loss.

The second parameter is "- $\mathrm{v}$ ", referring to the number of columns added for each toll path at each iteration of the column generation algorithm (see Section 4.7). Both the tuner and previous results show that it is better to add one column per iteration such that there is no ambiguity on the best value for this parameter.

Parameters "-s", "- $\mathrm{d}$ " and "-e" refer to the stabilisation strategy with a dual variable smoothing approach, detailed in Section 4.9.2. The tuner shows that strategies 4 and 5 perform better. Strategy 4 uses a fixed parameter $\Delta$, which best estimate is around 0.6 for complete and partial graph and A1 instances, around 0.3 for Shioda et al. instances, and around 0.55 if tuned on the mixed set of instances. Strategy 5 is the auto-adaptive scheme using the subgradient information to update the parameter $\Delta$ at each iteration of the column generation algorithm. As the best $\Delta$ value for strategy 4 is different for each class of instances and strategy 5 does not require to choose it at all, the best solution is to use strategy 5. Furthermore, having a best value of around 0.5 for Strategy 4 is in line with the value used by the auto-adaptive scheme, which starts at 0.5 and increases or decreases it accordingly to the subgradient. Parameter " $\mathrm{e}$ " represents the $\bar{\varepsilon}$ value used to stop the stabilisation when the primal/dual gap is small, and its best value is not clearly estimated by the tuning. We decide to use a value of 0.05 from the first configuration of Table 4.12, which gave good results for all instances. Overall the best configurations for the stabilisation found by the tuner comply with previous results (see Section 4.9.3).

As explained just above in Section 4.10.1, parameter "-m" allows SCIP to eventually remove columns that are outside of a basis for "-a" iterations. Tuning results show that this option is not useful, apart from few cases. In the configurations where this parameter is estimated to be one, then the best estimate of the number of out of base iterations before a column is deleted is quite high, ranging from 30 to 50 , which is often close to the number of iterations needed for the column generation algorithm to find the optimal solution. Therefore in these cases removing columns does not have much impact and not using this feature seems a good choice.

The last two parameters "-S" and "-R" choose the simplex algorithm to solve the linear problems, as explained in Section 4.10.1. The best algorithm for resolving LP relaxations if a starting basis exists ("- $R$ " parameter) is without doubts the dual simplex, whilst for initial LP relaxations ("-S" parameter) the best choice is not clear. Therefore we choose to use the automatic configuration for "-S" parameter, but in any case it will be used only once at the beginning of the solving, such that the choice is not very relevant.

According to the analysis, we define the best configuration of parameters as:

$$
-\mathrm{c}=2 \quad-\mathrm{v}=1 \quad-\mathrm{s}=5 \quad-\mathrm{e}=0.05 \quad-\mathrm{m}=0 \quad-\mathrm{S}=0 \quad-\mathrm{R}=2
$$




\subsubsection{Numerical results}

In this section we report numerical results for solving (HPDW-LR) with the basic column generation algorithm (noted as "BASIC", with $-\mathrm{c}=1,-\mathrm{v}=1,-\mathrm{s}=0,-\mathrm{m}=0,-\mathrm{S}=0,-\mathrm{R}=0$ ) and with the additional features discussed in this chapter and the best configuration of parameters discussed in the previous section (noted as "BEST", with $-\mathrm{c}=2,-\mathrm{v}=1,-\mathrm{s}=5$, $-\mathrm{e}=0.05,-\mathrm{m}=0,-\mathrm{S}=0,-\mathrm{R}=2$ ).

In Tables 4.13 and 4.14 we report, for both configurations, the time in seconds to find the optimal relaxed solution of (HPDW), and separately to solve the (MP) and the (SP). Furthermore, we report the number of columns generated during the solving and the number of iterations of the algorithm, which is the number of times the (MP) and (SP) are solved.

\begin{tabular}{ll|ccc|ccc|ccc}
\multicolumn{2}{l}{$\begin{array}{l}\text { Complete } \\
\text { graph }\end{array}$} & \multicolumn{3}{|c}{20 commodities } & \multicolumn{3}{c|}{56 commodities } & \multicolumn{3}{c}{90 commodities } \\
\hline BASIC & Time & 0.199 & 0.726 & 1.57 & 4.13 & 12.7 & 22.3 & 25.84 & 62.26 & 108.99 \\
& Cols & 335 & 655 & 924 & 1141 & 1765 & 2227 & 2103 & 3034 & 3749 \\
& Iters & 21 & 15 & 14 & 63 & 37 & 29 & 111 & 61 & 47 \\
(MP) & Time & 0.072 & 0.206 & 0.41 & 2.926 & 8.197 & 13.36 & 22.05 & 48.84 & 81.3 \\
(SP) & Time & 0.116 & 0.509 & 1.135 & 1.159 & 4.401 & 8.863 & 3.669 & 13.27 & 27.46 \\
\hline BEST & Time & 0.14 & 0.48 & 0.99 & 2.13 & 7.36 & 14.70 & 10.90 & 34.85 & 72.09 \\
& Cols & 331 & 707 & 1027 & 890 & 1488 & 1951 & 1503 & 2243 & 2912 \\
& Iters & 19 & 14 & 13 & 51 & 30 & 25 & 81 & 46 & 37 \\
(MP) & Time & 0.06 & 0.14 & 0.25 & 1.44 & 4.42 & 8.55 & 8.62 & 25.68 & 52.89 \\
(SP) & Time & 0.07 & 0.34 & 0.74 & 0.70 & 2.94 & 6.15 & 2.27 & 9.17 & 19.20 \\
\hline \hline Partial & & 20 commodities & 56 commodities & 90 & commodities \\
graph & & $20 \mathrm{a}$ & $56 \mathrm{a}$ & $90 \mathrm{a}$ & $20 \mathrm{a}$ & $56 \mathrm{a}$ & $90 \mathrm{a}$ & $20 \mathrm{a}$ & $56 \mathrm{a}$ & $90 \mathrm{a}$ \\
\hline BASIC & Time & 0.171 & 0.687 & 1.443 & 3.026 & 10.99 & 21.23 & 18.54 & 55.71 & 102.87 \\
& Cols & 301 & 625 & 891 & 974 & 1699 & 2199 & 1853 & 2926 & 3747 \\
& Iters & 19 & 15 & 13 & 55 & 35 & 29 & 100 & 58 & 47 \\
(MP) & Time & 0.063 & 0.204 & 0.387 & 1.832 & 6.81 & 12.63 & 15.27 & 42.99 & 76.26 \\
(SP) & Time & 0.101 & 0.474 & 1.04 & 1.16 & 4.135 & 8.52 & 3.176 & 12.56 & 26.4 \\
\hline BEST & Time & 0.10 & 0.45 & 0.92 & 1.57 & 5.93 & 11.79 & 7.32 & 25.99 & 53.90 \\
& Cols & 313 & 701 & 1013 & 759 & 1362 & 1829 & 1237 & 2004 & 2684 \\
& Iters & 17 & 14 & 12 & 42 & 28 & 23 & 69 & 41 & 34 \\
(SP) & Time & 0.04 & 0.13 & 0.21 & 1.00 & 3.31 & 6.25 & 5.41 & 17.98 & 36.79 \\
& Time & 0.05 & 0.32 & 0.71 & 0.58 & 2.62 & 5.54 & 1.91 & 8.02 & 17.11
\end{tabular}

Table 4.13: Numerical results on the best configuration of the column generation algorithm for the linear relaxation of (HPDW) Complete and partial graph instances

We notice that the best configuration of the algorithm allows us to reduce the computational time by about a factor of two for complete and partial graph instances, by a factor from three to seven for Shioda et al. instances and by about a factor of one third for A1 instances. The number of iterations is also significantly reduced (by 15 to $40 \%$ ), and if we use this number to calculate the average time to solve each (MP) and (SP) 


\begin{tabular}{|c|c|c|c|c|c|c|c|c|c|c|}
\hline \multirow{2}{*}{\multicolumn{2}{|c|}{$\begin{array}{l}\text { Shioda et al. } \\
\text { instances }\end{array}$}} & \multicolumn{3}{|c|}{40 commodities } & \multicolumn{3}{|c|}{60 commodities } & \multicolumn{3}{|c|}{80 commodities } \\
\hline & & $20 \mathrm{a}$ & $40 \mathrm{a}$ & $60 \mathrm{a}$ & $20 \mathrm{a}$ & $40 \mathrm{a}$ & $60 \mathrm{a}$ & $20 \mathrm{a}$ & $40 \mathrm{a}$ & $60 \mathrm{a}$ \\
\hline \multirow[t]{3}{*}{ BASIC } & Time & 5.51 & 7.79 & 11.39 & 43.12 & 47.04 & 56.83 & 198.38 & 221.28 & 260.09 \\
\hline & Cols & 1413 & 1714 & 2015 & 2721 & 3046 & 3506 & 4586 & 5130 & 5645 \\
\hline & Iters & 82 & 50 & 40 & 165 & 91 & 69 & 277 & 153 & 110 \\
\hline$(\mathrm{MP})$ & Time & 4.37 & 5.37 & 7.26 & 39.76 & 40.27 & 45.13 & 190.54 & 205.3 & 233 \\
\hline (SP) & Time & 1.1 & 2.37 & 4.07 & 3.23 & 6.62 & 11.53 & 7.49 & 15.66 & 26.71 \\
\hline \multirow[t]{3}{*}{ BEST } & Time & 1.84 & 3.15 & 5.18 & 7.82 & 13.84 & 21.76 & 28.76 & 53.03 & 71.49 \\
\hline & Cols & 1086 & 1401 & 1710 & 1765 & 2242 & 2498 & 2779 & 3240 & 3837 \\
\hline & Iters & 63 & 39 & 33 & 102 & 67 & 50 & 159 & 97 & 76 \\
\hline (MP) & Time & 1.24 & 1.76 & 2.66 & 6.13 & 9.87 & 15.09 & 24.80 & 44.16 & 55.77 \\
\hline$(\mathrm{SP})$ & Time & 0.60 & 1.38 & 2.52 & 1.69 & 3.96 & 6.67 & 3.96 & 8.87 & 15.71 \\
\hline \multirow{2}{*}{\multicolumn{2}{|c|}{$\begin{array}{l}\text { A1 } \\
\text { instances }\end{array}$}} & \multicolumn{3}{|c|}{21 commodities } & \multicolumn{3}{|c|}{55 commodities } & \multicolumn{3}{|c|}{91 commodities } \\
\hline & & $21 \mathrm{a}$ & $55 \mathrm{a}$ & $91 \mathrm{a}$ & $21 \mathrm{a}$ & $55 \mathrm{a}$ & $91 \mathrm{a}$ & $21 \mathrm{a}$ & $55 \mathrm{a}$ & $91 \mathrm{a}$ \\
\hline \multirow[t]{3}{*}{ BASIC } & Time & 0.07 & 0.22 & 0.46 & 0.48 & 1.69 & 3.85 & 3.14 & 8.63 & 16.45 \\
\hline & Cols & 137 & 333 & 460 & 347 & 572 & 863 & 727 & 1185 & 1527 \\
\hline & Iters & 11 & 12 & 11 & 30 & 21 & 19 & 59 & 39 & 29 \\
\hline (MP) & Time & 0.02 & 0.04 & 0.07 & 0.16 & 0.34 & 0.64 & 1.67 & 2.69 & 4.37 \\
\hline$(\mathrm{SP})$ & Time & 0.04 & 0.17 & 0.40 & 0.32 & 1.35 & 3.21 & 1.47 & 5.94 & 12.08 \\
\hline \multirow[t]{3}{*}{ BEST } & Time & 0.05 & 0.23 & 0.40 & 0.39 & 1.37 & 2.99 & 1.89 & 6.07 & 11.72 \\
\hline & Cols & 162 & 381 & 521 & 348 & 594 & 843 & 627 & 1030 & 1331 \\
\hline & Iters & 12 & 11 & 9 & 28 & 18 & 16 & 50 & 32 & 24 \\
\hline (MP) & Time & 0.01 & 0.04 & 0.04 & 0.09 & 0.17 & 0.28 & 0.63 & 1.15 & 1.73 \\
\hline (SP) & Time & 0.03 & 0.20 & 0.36 & 0.30 & 1.20 & 2.72 & 1.26 & 4.92 & 9.99 \\
\hline
\end{tabular}

Table 4.14: Numerical results on the best configuration of the column generation algorithm for the linear relaxation of (HPDW) Shioda et al. and A1 instances

we can see that the (MP) solution time is reduced, passing from 1.7 to 1.5 seconds for complete graph instances, from 1.6 to 1 second for partial graph instances, from 2 to 0.7 seconds for Shioda et al. instances and from 0.15 to 0.07 seconds for A1 instances, for the bigger instances of each type. The (SP) solution time remains constant, equal to 0.5 seconds for complete and partial graph and A1 instances and to 0.2 seconds for Shioda et al. instances. Finally the number of generated columns is also reduced in the best parameters configuration, by 15 to $40 \%$.

In Figure 4.14 we report the performance profile graphs for the time to solve (HPDWLR) with the basic version of the column generation algorithm and with the best one. We notice that the best algorithm version performs significantly better, especially for Shioda et al. instances, where we are able to solve all instances in less than $100 \mathrm{sec}-$ onds, whilst with the basic version within this time limit we solved only 60 instances ( $2 / 3$ of the total) and to solve all of them we needed 400 seconds. We also notice that, as we already revealed for the basic configuration of the algorithm, also the best one maintains a "step" shape for the complete graph instances, grouping the time needed to solve each instance in separated time windows accordingly to their size.

In Figure 4.15 we plot the evolution of the primal and dual bound during the solving 


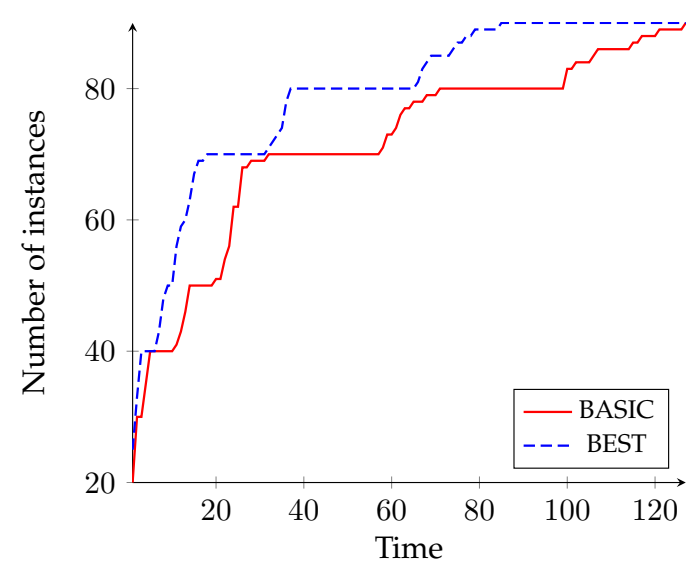

(a) Complete graph instances

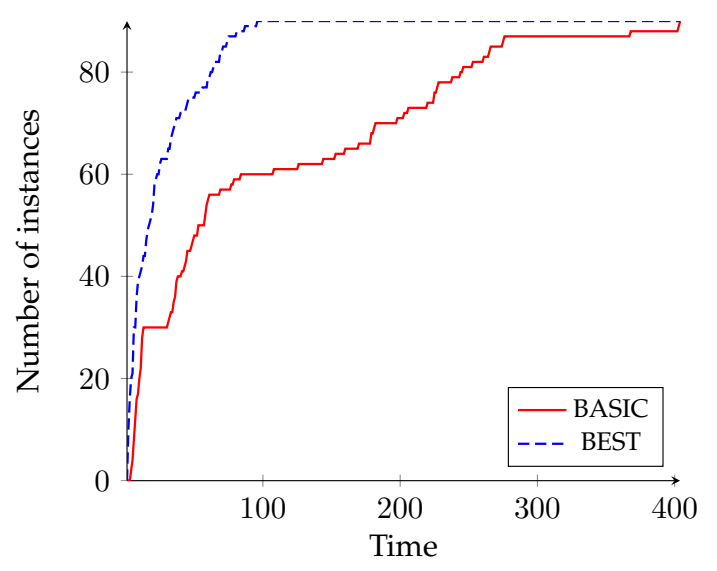

(c) Shioda et al.'s instances

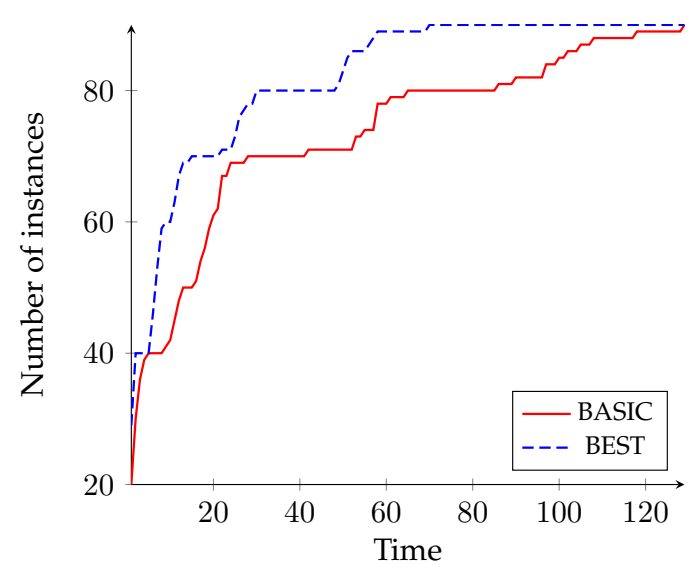

(b) Partial graph instances

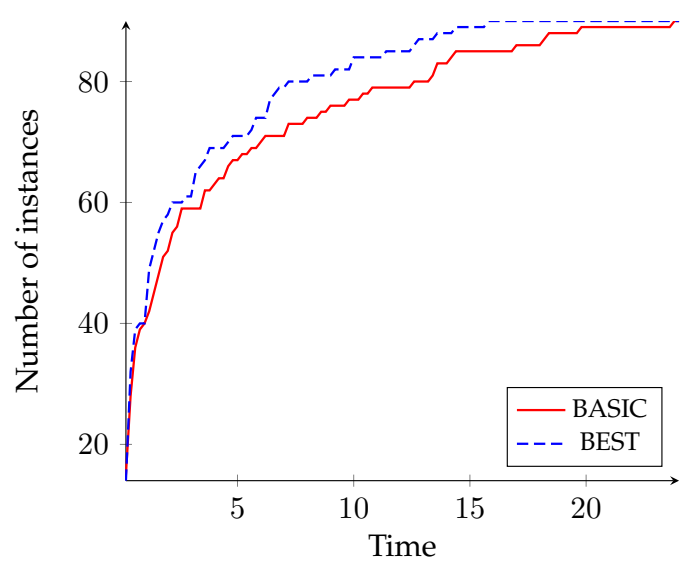

(d) A1 instances

Figure 4.14: Performance profile graphs on the best column generation algorithm for (HPDW-LR)

of one instance with the basic and the best versions of the column generation algorithm. We notice a significant reduction in the convergence time, especially for the Shioda et al. instance (see Figure 4.15(b)). For both instances the tailing-off effect and the oscillatory behaviour present in the basic version of the algorithm and described in Section 4.3.5 have been eliminated, such that all the improvements added to the algorithm reveal to be effective.

The numerical results for this best configuration of the column generation algorithm presented in this section confirm that the choice of the parameters values provided by the automatic tuning is very good. Furthermore they comply with partial numerical results that we performed for each feature of the algorithm, with the advantage of being done automatically in one run of tests.

In Table 4.15 we report the ratio of the solution time of (HPL-LR) formulation divided by the solution time of (HPDW-LR) formulation, with the basic and best configuration of the column generation algorithm: if the ratio is greater than one it means that (HPDW) is performing better, if it is less than one then (HPL) is better. This ratio, 


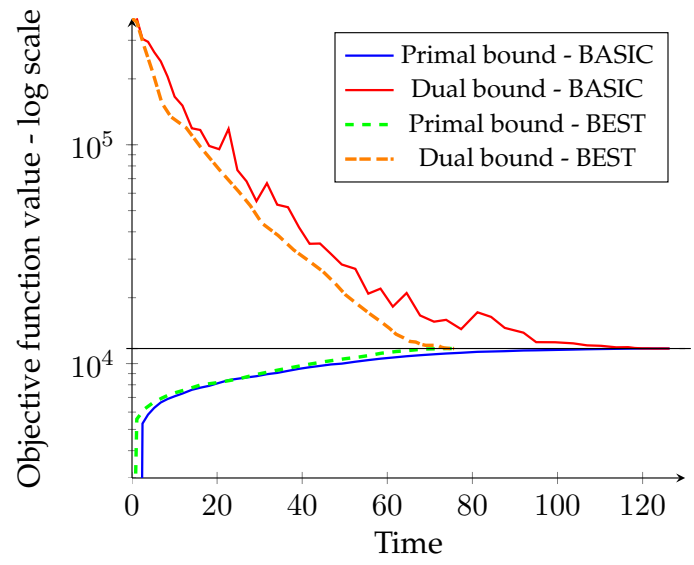

(a) 90k 90a complete graph instance

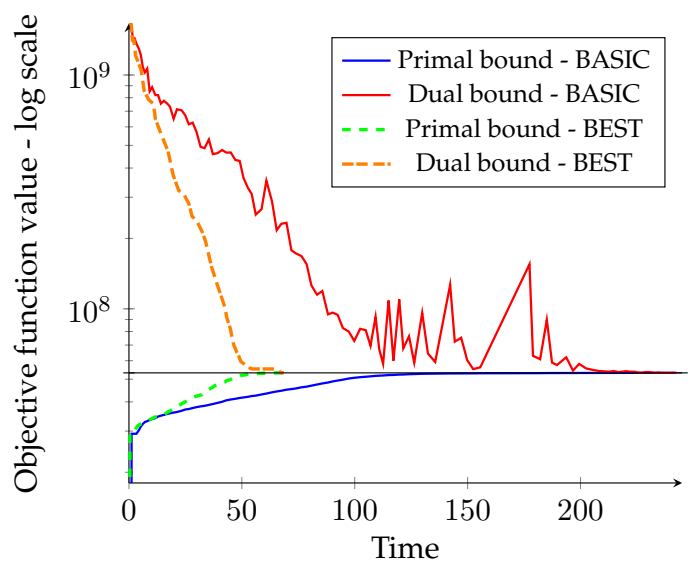

(b) $80 \mathrm{k} 60 \mathrm{a}$ Shioda et al. instance

Figure 4.15: Primal and dual bounds evolution while solving the linear relaxation of (HPDW) with the best configuration of the column generation

for the solution time of the basic column generation algorithm and as well for the linear relaxation gap, was already reported in Section 4.5.2. where the gap of (HPDW-LR) showed to be significantly better, but (HPL-LR) was much better performing regarding the solution time, apart from A1 instances.

\begin{tabular}{|c|c|c|c|c|c|c|c|c|c|c|}
\hline \multirow{2}{*}{$\begin{array}{l}\text { Complete } \\
\text { graph }\end{array}$} & & \multicolumn{3}{|c|}{20 commodities } & \multicolumn{3}{|c|}{56 commodities } & \multicolumn{3}{|c|}{90 commodities } \\
\hline & & $20 \mathrm{a}$ & $56 a$ & $90 \mathrm{a}$ & $20 \mathrm{a}$ & $56 a$ & $90 \mathrm{a}$ & $20 \mathrm{a}$ & $56 a$ & $90 \mathrm{a}$ \\
\hline (-L)/(-DW-basic) & Time & 0.2 & 0.4 & 0.5 & 0.04 & 0.1 & 0.2 & 0.02 & 0.1 & 0.1 \\
\hline (-L)/(-DW-best) & Time & 0.3 & 0.6 & 0.8 & 0.1 & 0.2 & 0.3 & 0.04 & 0.14 & 0.1 \\
\hline Partial & & \multicolumn{3}{|c|}{20 commodities } & \multicolumn{3}{|c|}{56 commodities } & \multicolumn{3}{|c|}{90 commodities } \\
\hline graph & & $20 \mathrm{a}$ & $56 a$ & $90 \mathrm{a}$ & $20 \mathrm{a}$ & $56 a$ & $90 \mathrm{a}$ & $20 \mathrm{a}$ & $56 \mathrm{a}$ & $90 \mathrm{a}$ \\
\hline (-L)/(-DW-basic) & Time & 0.2 & 0.4 & 0.6 & 0.1 & 0.2 & 0.3 & 0.03 & 0.1 & 0.1 \\
\hline (-L)/(-DW-best) & Time & 0.4 & 0.6 & 0.9 & 0.1 & 0.3 & 0.5 & 0.1 & 0.14 & 0.2 \\
\hline Shioda et al. & & \multicolumn{3}{|c|}{40 commodities } & \multicolumn{3}{|c|}{60 commodities } & \multicolumn{3}{|c|}{80 commodities } \\
\hline instances & & $20 \mathrm{a}$ & $40 \mathrm{a}$ & $60 \mathrm{a}$ & $20 \mathrm{a}$ & $40 \mathrm{a}$ & $60 \mathrm{a}$ & $20 a$ & $40 \mathrm{a}$ & $60 \mathrm{a}$ \\
\hline (-L)/(-DW-basic) & Time & 0.02 & 0.03 & 0.04 & 0.01 & 0.01 & 0.01 & 0.001 & 0.002 & 0.0004 \\
\hline (-L)/(-DW-best) & Time & 0.05 & 0.07 & 0.1 & 0.02 & 0.03 & 0.04 & 0.01 & 0.01 & 0.02 \\
\hline A1 & & \multicolumn{3}{|c|}{21 commodities } & \multicolumn{3}{|c|}{55 commodities } & \multicolumn{3}{|c|}{91 commodities } \\
\hline instances & & $21 \mathrm{a}$ & $55 \mathrm{a}$ & $91 \mathrm{a}$ & $21 \mathrm{a}$ & $55 \mathrm{a}$ & $91 \mathrm{a}$ & $21 \mathrm{a}$ & $55 \mathrm{a}$ & $91 \mathrm{a}$ \\
\hline (-L)/(-DW-basic) & Time & 1.9 & 2.2 & 2.6 & 1.0 & 1.4 & 1.5 & 0.4 & 0.7 & 0.9 \\
\hline (-L)/(-DW-best) & Time & 2.6 & 2.1 & 3.0 & 1.3 & 1.7 & 1.9 & 0.7 & 0.96 & 1.3 \\
\hline
\end{tabular}

Table 4.15: Performance ratio of the basic and the best configurations of the column generation algorithm for (HPDW-LR) formulation with respect to (HPL-LR) formulation

We notice that using the best configuration of parameters the relative performance of (HPDW-LR) improve significantly. In particular for A1 instances now (HPDW-LR) is almost always better than (HPL-LR), being three times quicker for instances with 21 commodities and 91 toll paths. Furthermore, for complete and partial graph instances, 
the speed of the two models is now comparable when there are 20 commodities and 90 toll paths. The ratio trend is same for both the basic and best configurations of the column generation algorithm, increasing when the number of toll paths increases and decreasing when the number of commodities increases.

\subsection{Valid inequalities}

In Section 1.5.8 we introduced two families of valid inequalities for the NPP with connected toll arcs, proposed by Heilporn et al. (2011) to strengthened shortest path constraints $(1.17 \mathrm{~b})$ and $(1.17 \mathrm{c}$. They are called "Strengthened Shortest Path Inequalities" (SSPI) and, for any subset $\mathcal{S}$ of $\mathcal{A}$, for any path $b \in \mathcal{A}$ and any pair of commodities $k_{1}, k_{2} \in \mathcal{K} \times \mathcal{K}$, are expressed as follows:

$$
\begin{aligned}
& \sum_{a \in \mathcal{A}_{k_{1}}}\left(c_{a}^{k_{1}} x_{a}^{k_{1}}+p_{a}^{k_{1}}\right)+c_{o d}^{k_{1}}\left(1-\sum_{a \in \mathcal{A}_{k_{1}}} x_{a}^{k_{1}}\right) \leq c_{b}^{k_{1}}+T_{b}+\sum_{a \in \mathcal{A} \backslash(\mathcal{S} \cup\{b\})}\left[p_{a}^{k_{2}}+\left(c_{a}^{k_{1}}-c_{b}^{k_{1}}\right) x_{a}^{k_{2}}\right] \\
& \sum_{a \in \mathcal{A}_{k_{1}}}\left(c_{a}^{k_{1}} x_{a}^{k_{1}}+p_{a}^{k_{1}}\right)+c_{o d}^{k_{1}}\left(1-\sum_{a \in \mathcal{A}_{k_{1}}} x_{a}^{k_{1}}\right) \leq c_{o d}^{k_{1}}+\sum_{a \in \mathcal{A} \backslash \mathcal{S}}\left[p_{a}^{k_{2}}+\left(c_{a}^{k_{1}}-c_{o d}^{k_{1}}\right) x_{a}^{k_{2}}\right]
\end{aligned}
$$

These inequalities are still valid for our (HPDW) reformulation and we now detail how they can be included in the model.

\subsubsection{Modified master problem}

Remember the relation between the two spaces of variables: $x_{a}^{k}=\sum_{j \in \mathcal{J}} \lambda_{a}^{j} x_{a}^{k, j}, T_{a}=$ $\sum_{j \in \mathcal{J}} \lambda_{a}^{j} T_{a}^{j}$ and $p_{a}^{k}=\sum_{j \in \mathcal{J}} \lambda_{a}^{j} p_{a}^{k, j}$. Therefore, using variables $\lambda_{a}^{j}$, (SSPI) can be reformulated as follows:

$$
\begin{aligned}
\sum_{a \in \mathcal{A}}\left(c_{a}^{k_{1}} \sum_{j \in \mathcal{J}} \lambda_{a}^{j} x_{a}^{k_{1}, j}+\sum_{j \in \mathcal{J}} \lambda_{a}^{j} p_{a}^{k_{1}, k}\right) & +c_{o d}^{k_{1}}\left(1-\sum_{a \in \mathcal{A}_{k_{1}}} \sum_{j \in \mathcal{J}} \lambda_{a}^{j} x_{a}^{k_{1}, j}\right) \leq c_{b}^{k_{1}}+\sum_{j \in \mathcal{J}} \lambda_{b}^{j} T_{b}^{j}+ \\
& +\sum_{a \in \mathcal{A} \backslash(\mathcal{S} \cup\{b\})}\left[\sum_{j \in \mathcal{J}} \lambda_{a}^{j} p_{a}^{k_{2}, j}+\left(c_{a}^{k_{1}}-c_{b}^{k_{1}}\right) \sum_{j \in \mathcal{J}} \lambda_{a}^{j} x_{a}^{k_{2}, j}\right] \text { (4.55a) } \\
\sum_{a \in \mathcal{A}}\left(c_{a}^{k_{1}} \sum_{j \in \mathcal{J}} \lambda_{a}^{j} x_{a}^{k_{1}, j}+\sum_{j \in \mathcal{J}} \lambda_{a}^{j} p_{a}^{k_{1}, k}\right) & +c_{o d}^{k_{1}}\left(1-\sum_{a \in \mathcal{A}_{k_{1}}} \sum_{j \in \mathcal{J}} \lambda_{a}^{j} x_{a}^{k_{1}, j}\right) \leq c_{o d}^{k_{1}}+ \\
& +\sum_{a \in \mathcal{A} \backslash \mathcal{S}}\left[\sum_{j \in \mathcal{J}} \lambda_{a}^{j} p_{a}^{k_{2}, j}+\left(c_{a}^{k_{1}}-c_{o d}^{k_{1}}\right) \sum_{j \in \mathcal{J}} \lambda_{a}^{j} x_{a}^{k_{2}, j}\right]
\end{aligned}
$$

Rearranging terms and substituting costs of paths with $M$ parameters (remember $M_{a}^{k}=\max \left\{0, c_{o d}^{k}-c_{a}^{k}\right\}$, see Section 1.5.7, we obtain the following inequalities: 


$$
\begin{array}{r}
\sum_{a \in \mathcal{S}^{\prime}} \sum_{j \in \mathcal{J}} \lambda_{a}^{j}\left[p_{a}^{k_{1}, j}-M_{a}^{k_{1}} x_{a}^{k_{1}, j}-\left(M_{b}^{k_{1}}-M_{a}^{k_{1}}\right) x_{a}^{k_{2}, j}-p_{a}^{k_{2}, j}\right]+ \\
+\sum_{a \notin \mathcal{S}^{\prime}} \sum_{j \in \mathcal{J}} \lambda_{a}^{j}\left(p_{a}^{k_{1}, j}-M_{a}^{k_{1}} x_{a}^{k_{1}, j}\right) \\
-\sum_{j \in \mathcal{J}} \lambda_{b}^{j} T_{b}^{j} \leq-M_{b}^{k_{1}} \\
\sum_{a \in \mathcal{S}^{\prime \prime}} \sum_{j \in \mathcal{J}} \lambda_{a}^{j}\left(p_{a}^{k_{1}, j}-M_{a}^{k_{1}} x_{a}^{k_{1}, j}+M_{a}^{k_{1}} x_{a}^{k_{2}, j}-p_{a}^{k_{2}, j}\right)+\sum_{a \notin \mathcal{S}^{\prime \prime}} \sum_{j \in \mathcal{J}} \lambda_{a}^{j}\left(p_{a}^{k_{1}, j}-M_{a}^{k_{1}} x_{a}^{k_{1}, j}\right) \leq 0
\end{array}
$$

where $\mathcal{S}^{\prime}=\mathcal{A} \backslash(\mathcal{S} \cup\{b\})$ and $\mathcal{S}^{\prime \prime}=\mathcal{A} \backslash \mathcal{S}$. These sets are constructed using the same polynomial separation procedure described in Section 1.5.8. These inequalities are added to the (MP) introduced in Section 4.3.1.

\subsubsection{Modified subproblem}

Introducing these inequalities in the (MP) has an impact on the (SP) described in Section 4.3.2. We associate dual variables to (SSPI) constraints as follows:

- variables $\delta_{b, \mathcal{S}^{\prime}}^{k_{1}, k_{2}} \geq 0$ are associated to constraints $4.56 a$, one for each inequality and corresponding $b \in \mathcal{A}, k_{1} \in \mathcal{K}, k_{2} \in \mathcal{K}$ and subset $\mathcal{S}^{\prime} \in \mathcal{A}$;

- variables $\delta_{o d, \mathcal{S}^{\prime \prime}}^{k_{1}, k_{2}} \geq 0$ are associated to constraints $4.56 \mathrm{~b}$, one for each inequality and corresponding $k_{1} \in \mathcal{K}, k_{2} \in \mathcal{K}$ and subset $\mathcal{S}^{\prime \prime} \in \mathcal{A}$.

The reduced price of a variable $\lambda_{a}^{j}$ of the (MP) is therefore modified as follows:

$$
\begin{aligned}
R P_{a}= & \sum_{k \in \mathcal{K}} \eta^{k} p_{a}^{k}-\left\{\sum_{k \in \mathcal{K}} \sum_{b \in \mathcal{A}} \delta_{b}^{k}\left(p_{a}^{k}-M_{a}^{k} x_{a}^{k}\right)-\sum_{k \in \mathcal{K}} \delta_{a}^{k} T_{a}+\sum_{k \in \mathcal{K}} x_{a}^{k} \gamma^{k}+\mu_{a}+\right. \\
& +\sum_{k_{1} \in \mathcal{K}} \sum_{k_{2} \in \mathcal{K}} \sum_{b \in \mathcal{A}} \sum_{\mathcal{S}^{\prime}: a \in \mathcal{S}^{\prime}} \delta_{b, \mathcal{S}^{\prime}}^{k_{1}, k_{2}}\left[p_{a}^{k_{1}}-M_{a}^{k_{1}} x_{a}^{k_{1}}-p_{a}^{k_{2}}-\left(M_{b}^{k_{1}}-M_{a}^{k_{1}}\right) x_{a}^{k_{2}}\right]+ \\
& +\sum_{k_{1} \in \mathcal{K}} \sum_{k_{2} \in \mathcal{K}} \sum_{b \in \mathcal{A}} \sum_{\mathcal{S}^{\prime}: a \notin \mathcal{S}^{\prime}} \delta_{b, \mathcal{S}^{\prime}}^{k_{1}, k_{2}}\left(p_{a}^{k_{1}}-M_{a}^{k_{1}} x_{a}^{k_{1}}\right)-\sum_{k_{1} \in \mathcal{K}} \sum_{k_{2} \in \mathcal{K}} \sum_{\mathcal{S}^{\prime}} \delta_{a, \mathcal{S}^{\prime}}^{k_{1}, k_{2}} T_{a}+ \\
& +\sum_{k_{1} \in \mathcal{K}} \sum_{k_{2} \in \mathcal{K}} \sum_{\mathcal{S}^{\prime \prime}: a \in \mathcal{S}^{\prime \prime}} \delta_{o d, \mathcal{S}^{\prime \prime}}^{k_{1}, k_{2}}\left(p_{a}^{k_{1}}-M_{a}^{k_{1}} x_{a}^{k_{1}}-p_{a}^{k_{2}}+M_{a}^{k_{1}} x_{a}^{k_{2}}\right)+ \\
& \left.+\sum_{k_{1} \in \mathcal{K}} \sum_{k_{2} \in \mathcal{K}} \sum_{\mathcal{S}^{\prime \prime}: a \notin \mathcal{S}^{\prime \prime}} \delta_{o d, \mathcal{S}^{\prime \prime}}^{k_{1}, k_{2}}\left(p_{a}^{k_{1}}-M_{a}^{k_{1}} x_{a}^{k_{1}}\right)\right\}
\end{aligned}
$$

Indicating explicitly the term associated to assignment variables, the reduced price is: 


$$
\begin{aligned}
R P_{a}= & \sum_{k_{1} \in \mathcal{K}} x_{a}^{k_{1}}\left[T _ { a } \left(\eta^{k_{1}}-\sum_{b \in \mathcal{A}} \delta_{b}^{k_{1}}-\sum_{k_{2} \in \mathcal{K}} \sum_{b \in \mathcal{A}} \sum_{\mathcal{S}^{\prime}} \delta_{b, \mathcal{S}^{\prime}}^{k_{1}, k_{2}}+\sum_{k_{2} \in \mathcal{K}} \sum_{b \in \mathcal{A}} \sum_{\mathcal{S}^{\prime}: a \in \mathcal{S}^{\prime}} \delta_{b, \mathcal{S}^{\prime}}^{k_{2}, k_{1}}+\right.\right. \\
& \left.-\sum_{k_{2} \in \mathcal{K}} \sum_{\mathcal{S}^{\prime \prime}} \delta_{o d, \mathcal{S}^{\prime \prime}}^{k_{1}, k_{2}}+\sum_{k_{2} \in \mathcal{K}} \sum_{\mathcal{S}^{\prime \prime}: a \in \mathcal{S}^{\prime \prime}} \delta_{o d, \mathcal{S}^{\prime \prime}}^{k_{2}, k_{1}}\right)+\sum_{b \in \mathcal{A}} M_{a}^{k_{1}} \delta_{b}^{k_{1}}+\sum_{k_{2} \in \mathcal{K}} \sum_{b \in \mathcal{A}} \sum_{\mathcal{S}^{\prime}} M_{a}^{k_{1}} \delta_{b, \mathcal{S}^{\prime}}^{k_{1}, k_{2}}+ \\
& +\sum_{k_{2} \in \mathcal{K}} \sum_{b \in \mathcal{A}} \sum_{\mathcal{S}^{\prime}: a \in \mathcal{S}^{\prime}}\left(M_{b}^{k_{2}}-M_{a}^{k_{2}}\right) \delta_{b, \mathcal{S}^{\prime}}^{k_{2}, k_{1}}+\sum_{k_{2} \in \mathcal{K}} \sum_{\mathcal{S}^{\prime \prime}} M_{a}^{k_{1}} \delta_{o d, \mathcal{S}^{\prime \prime}}^{k_{1}, k_{2}}+ \\
& \left.-\sum_{k_{2} \in \mathcal{K}} \sum_{\mathcal{S}^{\prime \prime}: a \in \mathcal{S}^{\prime \prime}} M_{a}^{k_{2}} \delta_{o d, \mathcal{S}^{\prime \prime}}^{k_{2}, k_{1}}-\gamma^{k_{1}}\right]+\sum_{k \in \mathcal{K}} \delta_{a}^{k} T_{a}+\sum_{k_{1} \in \mathcal{K}} \sum_{k_{2} \in \mathcal{K}} \sum_{\mathcal{S}^{\prime}} \delta_{a, \mathcal{S}^{\prime}}^{k_{1}, k_{2}} T_{a}-\mu_{a} \quad \text { (4.58a) }
\end{aligned}
$$

The ( $\left.\mathrm{SP}_{a}\right)$, for each $a \in \mathcal{A}$ described in Section 4.3.2 does not change nor its solution procedure, except that it maximises Equation 4.57a and that parameter $\pi_{a}^{k_{1}}$ is the coefficient that multiplies $x_{a}^{k_{1}}$ in Equation 4.58a).

\subsubsection{Modified dual bound for column generation}

Adding inequalities 4.56a modifies the dual upper bound introduced in Section 4.3.3 as follows:

$$
U B=\sum_{k \in \mathcal{K}} \gamma^{k}+\sum_{a \in \mathcal{A}} \mu_{a}-\sum_{b \in \mathcal{A}} \sum_{k \in \mathcal{K}} M_{b}^{k} \delta_{b}^{k}-\sum_{k_{1} \in \mathcal{K}} \sum_{k_{2} \in \mathcal{K}} \sum_{b \in \mathcal{A}} \sum_{\mathcal{S}^{\prime}} M_{b}^{k_{1}} \delta_{b, \mathcal{S}^{\prime}}^{k_{1}, k_{2}}+\sum_{a \in \mathcal{A}} \mathrm{RP}_{a}
$$

\subsubsection{Numerical results}

We tested the performance of the linear relaxation of the (HPDW) model when adding the (SSPI) constraints. Let us make a small note on the running of these tests: to allow for SCIP to take into account the possibility of adding cuts we need to solve the integer version of the problem. Therefore the results presented in this section have been obtained running the integer models with a node limit of one, providing us the results for the linear relaxation of the root node. The time spent by SCIP to create the first children and to stop the solving due to the node limit is not significant compared to the time needed to solve the root node.

First of all we consider adding at each iteration of the separation procedure one (SSPI) pair of constraints for each pair of commodities $k_{1}, k_{2}$ and toll path $b$, finding the sets $\mathcal{S}$ for which the violation is maximal. In Tables 4.16 and 4.17 we compare solving the linear relaxation of (HPDW) with and without adding (SSPI) constraints, for our four types of instances. We report the solution time in seconds, the number of generated columns and the number of iterations of the column generation algorithm for both strategies. Furthermore when adding (SSPI) constraints we report the time the algorithm spends for solving the (MP) and the (SP). These values were reported for the basic model in Table 4.1 and we do not include them here again for sake of space. We 
also report the time spent for separating (SSPI) and the number of inequalities (4.56a) and $4.56 \mathrm{~b}$ found, as "NumIneq1" and "NumIneq2" respectively.

\begin{tabular}{|c|c|c|c|c|c|c|c|c|c|c|}
\hline \multirow{2}{*}{$\begin{array}{l}\text { Complete } \\
\text { graph }\end{array}$} & & \multicolumn{3}{|c|}{20 commodities } & \multicolumn{3}{|c|}{56 commodities } & \multicolumn{3}{|c|}{90 commodities } \\
\hline & & $20 \mathrm{a}$ & $56 a$ & $90 \mathrm{a}$ & $20 \mathrm{a}$ & $56 \mathrm{a}$ & $90 \mathrm{a}$ & $20 \mathrm{a}$ & $56 \mathrm{a}$ & $90 \mathrm{a}$ \\
\hline \multirow[t]{3}{*}{ (HPDW) } & Time & 0.199 & 0.726 & 1.57 & 4.13 & 12.7 & 22.3 & 25.84 & 62.26 & 108.99 \\
\hline & Cols & 335 & 655 & 924 & 1141 & 1765 & 2227 & 2103 & 3034 & 3749 \\
\hline & Iters & 21 & 15 & 14 & 63 & 37 & 29 & 111 & 61 & 47 \\
\hline \multirow[t]{3}{*}{ +SSPI } & Time & 1.80 & 9.28 & 12.90 & 260.58 & 1676.80 & 2470.51 & 2824.31 & 17801.94 & 18003.98 \\
\hline & Cols & 604 & 992 & 1339 & 3547 & 4676 & 4918 & 7138 & 10469 & 10181 \\
\hline & Iters & 72 & 65 & 52 & 293 & 229 & 186 & 492 & 341 & 248 \\
\hline$(\mathrm{MP})$ & Time & 0.68 & 1.94 & 2.69 & 157.24 & 774.46 & 623.97 & 1957.47 & 11697.42 & 9021.57 \\
\hline$(\mathrm{SP})$ & Time & 1.09 & 7.14 & 9.80 & 102.94 & 899.73 & 1839.75 & 865.69 & 6097.58 & 8966.85 \\
\hline \multirow[t]{3}{*}{ (SSPI) } & SepaTime & 0.03 & 0.20 & 0.42 & 0.40 & 2.60 & 6.79 & 1.14 & 6.95 & 15.57 \\
\hline & NumIneq1 & 9 & 18 & 27 & 20 & 55 & 98 & 35 & 74 & 132 \\
\hline & NumIneq2 & 377 & 677 & 646 & 1825 & 4982 & 7142 & 3180 & 9233 & 13467 \\
\hline \multirow{2}{*}{\multicolumn{2}{|c|}{$\begin{array}{l}\text { Partial } \\
\text { graph }\end{array}$}} & \multicolumn{3}{|c|}{20 commodities } & \multicolumn{3}{|c|}{56 commodities } & \multicolumn{3}{|c|}{90 commodities } \\
\hline & & $20 \mathrm{a}$ & $56 \mathrm{a}$ & $90 \mathrm{a}$ & $20 \mathrm{a}$ & $56 \mathrm{a}$ & $90 \mathrm{a}$ & $20 \mathrm{a}$ & $56 \mathrm{a}$ & $90 \mathrm{a}$ \\
\hline \multirow[t]{3}{*}{ (HPDW) } & Time & 0.171 & 0.687 & 1.443 & 3.026 & 10.99 & 21.23 & 18.54 & 55.71 & 102.87 \\
\hline & Cols & 301 & 625 & 891 & 974 & 1699 & 2199 & 1853 & 2926 & 3747 \\
\hline & Iters & 19 & 15 & 13 & 55 & 35 & 29 & 100 & 58 & 47 \\
\hline \multirow[t]{3}{*}{+ SSPI } & Time & 3.72 & 26.29 & 80.56 & 320.96 & 4028.78 & $11667.26^{* 1}$ & 5645.97 & $18000^{* 5}$ & $18000^{* 8}$ \\
\hline & Cols & 690 & 1244 & 1730 & 3490 & 6308 & 7456 & 7365 & 11285 & 11554 \\
\hline & Iters & 91 & 87 & 98 & 287 & 302 & 289 & 508 & 311 & 207 \\
\hline (MP) & Time & 1.26 & 6.25 & 19.50 & 155.89 & 1640.32 & 5664.65 & 3861.96 & 9857.79 & 8857.53 \\
\hline$(\mathrm{SP})$ & Time & 2.42 & 19.73 & 60.08 & 164.57 & 2384.37 & 5991.41 & 1782.36 & 8136.72 & 9158.22 \\
\hline \multirow[t]{3}{*}{ (SSPI) } & SepaTime & 0.05 & 0.31 & 0.98 & 0.50 & 4.09 & 11.20 & 1.65 & 8.56 & 13.89 \\
\hline & NumIneq1 & 43 & 145 & 316 & 63 & 543 & 964 & 73 & 387 & 263 \\
\hline & NumIneq2 & 549 & 1237 & 1794 & 2624 & 8180 & 12253 & 5549 & 14819 & 17606 \\
\hline
\end{tabular}

Table 4.16: Numerical results on adding SSPI to the column generation algorithm for the linear relaxation of (HPDW) Complete and partial graph instances

We notice that adding (SSPI) increases a lot the solution time of the column generation algorithm. We fixed a time limit of 5 hours and some of the partial graph and A1 instances were not solved within it (* represents the number of instances not solved in the time limit). In particular we can see that the number of added inequalities is very big, increasing the size of the (MP) and also slowing down the solving of the (SP). For the bigger complete and partial graph instances, each (MP) and (SP) takes now in average over 30 seconds to be solved, whilst before the (MP) was solved in less than 2 seconds, and the (SP) in 0.5 seconds. We therefore decided to test another strategy for the cuts separation, searching for one (SSPI) pair of constraints for each commodity $k_{1}$ and toll path $b$, finding the commodity $k_{2}$ and the sets $\mathcal{S}$ for which the violation is maximal. In fact it might often happen that two inequalities with the same pair $k_{1}, b$ and different $k_{2}$ are very similar, such that adding only one is enough.

In Table 4.18 we report numerical results for this strategy, noted as "Str 2". The time 


\begin{tabular}{|c|c|c|c|c|c|c|c|c|c|c|}
\hline \multirow{2}{*}{\multicolumn{2}{|c|}{$\begin{array}{l}\text { Shioda et al. } \\
\text { instances }\end{array}$}} & \multicolumn{3}{|c|}{40 commodities } & \multicolumn{3}{|c|}{60 commodities } & \multicolumn{3}{|c|}{80 commodities } \\
\hline & & $20 \mathrm{a}$ & $40 \mathrm{a}$ & $60 \mathrm{a}$ & $20 \mathrm{a}$ & $40 \mathrm{a}$ & $60 a$ & $20 \mathrm{a}$ & $40 \mathrm{a}$ & $60 \mathrm{a}$ \\
\hline \multirow[t]{3}{*}{ (HPDW) } & Time & 5.51 & 7.79 & 11.39 & 43.12 & 47.04 & 56.83 & 198.38 & 221.28 & 260.09 \\
\hline & Cols & 1413 & 1714 & 2015 & 2721 & 3046 & 3506 & 4586 & 5130 & 5645 \\
\hline & Iters & 82 & 50 & 40 & 165 & 91 & 69 & 277 & 153 & 110 \\
\hline \multirow[t]{3}{*}{ +SSPI } & Time & 30.31 & 25.93 & 19.78 & 436.25 & 492.85 & 551.81 & 6692.59 & 1836.38 & 2805.57 \\
\hline & Cols & 2033 & 1981 & 2144 & 4590 & 4238 & 4506 & 8138 & 6793 & 6754 \\
\hline & Iters & 168 & 90 & 58 & 360 & 194 & 134 & 608 & 280 & 189 \\
\hline (MP) & Time & 23.62 & 14.98 & 11.42 & 386.06 & 391.39 & 403.22 & 6469.74 & 1566.55 & 2378.55 \\
\hline (SP) & Time & 6.58 & 10.72 & 8.03 & 49.86 & 100.77 & 147.23 & 221.99 & 268.63 & 424.90 \\
\hline \multirow[t]{3}{*}{ (SSPI) } & SepaTime & 0.11 & 0.23 & 0.32 & 0.34 & 0.69 & 1.36 & 0.86 & 1.20 & 2.12 \\
\hline & NumIneq1 & 13 & 7 & 6 & 7 & 16 & 189 & 177 & 17 & 69 \\
\hline & NumIneq2 & 482 & 564 & 415 & 903 & 1377 & 1683 & 1131 & 1529 & 2045 \\
\hline \multirow{2}{*}{\multicolumn{2}{|c|}{$\begin{array}{l}\text { A1 } \\
\text { instances }\end{array}$}} & \multicolumn{3}{|c|}{21 commodities } & \multicolumn{3}{|c|}{55 commodities } & \multicolumn{3}{|c|}{91 commodities } \\
\hline & & $21 \mathrm{a}$ & 55 a & $91 \mathrm{a}$ & $21 \mathrm{a}$ & $55 \mathrm{a}$ & $91 \mathrm{a}$ & $21 \mathrm{a}$ & $55 \mathrm{a}$ & $91 \mathrm{a}$ \\
\hline \multirow[t]{3}{*}{ (HPDW) } & Time & 0.07 & 0.22 & 0.46 & 0.48 & 1.69 & 3.85 & 3.14 & 8.63 & 16.45 \\
\hline & Cols & 137 & 333 & 460 & 347 & 572 & 863 & 727 & 1185 & 1527 \\
\hline & Iters & 11 & 12 & 11 & 30 & 21 & 19 & 59 & 39 & 29 \\
\hline \multirow[t]{3}{*}{+ SSPI } & Time & 0.50 & 87.02 & 12.85 & 18.83 & 107.15 & 213.77 & $2249.15^{* 1}$ & $3852.73^{* 1}$ & $9867.31^{* 3}$ \\
\hline & Cols & 193 & 436 & 500 & 636 & 809 & 1085 & 2438 & 2589 & 2743 \\
\hline & Iters & 33 & 53 & 33 & 121 & 86 & 63 & 374 & 283 & 219 \\
\hline (MP) & Time & 0.11 & 8.55 & 0.54 & 2.71 & 3.93 & 5.55 & 1208.88 & 1124.51 & 1065.55 \\
\hline (SP) & Time & 0.36 & 78.20 & 11.95 & 15.96 & 102.02 & 205.69 & 1039.45 & 2723.58 & 8787.61 \\
\hline \multirow[t]{3}{*}{ (SSPI) } & SepaTime & 0.02 & 0.27 & 0.37 & 0.17 & 1.20 & 2.54 & 0.82 & 4.64 & 14.16 \\
\hline & NumIneq1 & 106 & 1961 & 837 & 239 & 1224 & 2263 & 914 & 2666 & 8585 \\
\hline & NumIneq2 & 88 & 215 & 97 & 343 & 464 & 400 & 1043 & 1763 & 2040 \\
\hline
\end{tabular}

Table 4.17: Numerical results on adding SSPI to the column generation algorithm for the linear relaxation of (HPDW) Shioda et al. and A1 instances

spent by the separation algorithm was not relevant for the first strategy (see Tables 4.16 and 4.17) and it is the same for the second strategy, such that we omit it.

We notice that the solution time is significantly reduced with this second separation strategy, but large partial graph instances still take a lot of time to be solved (up to almost 8000 seconds, with about 8-9 seconds for each (MP) and (SP)). The number of added inequalities is also significantly reduced, especially for the second type of inequalities.

In Tables 4.19 and 4.20 we compare the gap improvement for (HPL) and (HPDW) formulations when adding (SSPI), with both separation strategies. We report the gap between the optimal integer value and the linear relaxation optimal value and the time to solve the linear relaxation in seconds. The performances of both formulations and all three strategies are shown in Figure 4.16, where we report the performance profile graphs for the gap between the optimal integer value and the linear relaxation optimal value. The ordinate's axis represents the number of instances for which the gap is 


\begin{tabular}{|c|c|c|c|c|c|c|c|c|c|c|}
\hline \multirow{2}{*}{\multicolumn{2}{|c|}{$\begin{array}{l}\text { Complete } \\
\text { graph }\end{array}$}} & \multicolumn{3}{|c|}{20 commodities } & \multicolumn{3}{|c|}{56 commodities } & \multicolumn{3}{|c|}{90 commodities } \\
\hline & & $20 \mathrm{a}$ & $56 \mathrm{a}$ & $90 \mathrm{a}$ & $20 \mathrm{a}$ & 56 a & $90 \mathrm{a}$ & $20 \mathrm{a}$ & $56 \mathrm{a}$ & $90 \mathrm{a}$ \\
\hline +SSPI & Time & 0.70 & 1.92 & 4.59 & 35.51 & 105.03 & 188.44 & 225.14 & 470.59 & 1152.23 \\
\hline \multirow[t]{2}{*}{ Str 2} & Cols & 456 & 787 & 1124 & 2234 & 2810 & 3272 & 4306 & 4970 & 6003 \\
\hline & Iters & 57 & 43 & 48 & 198 & 148 & 138 & 329 & 212 & 198 \\
\hline$(\mathrm{MP})$ & Time & 0.32 & 0.66 & 1.44 & 25.14 & 47.47 & 72.21 & 172.31 & 251.32 & 487.47 \\
\hline$(\mathrm{SP})$ & Time & 0.34 & 1.11 & 2.66 & 10.09 & 55.14 & 108.90 & 51.98 & 212.73 & 644.94 \\
\hline \multirow[t]{2}{*}{ (SSPI) } & NumIneq1 & 1 & 0 & 0 & 0 & 2 & 2 & 0 & 0 & 1 \\
\hline & NumIneq2 & 78 & 85 & 121 & 274 & 547 & 659 & 409 & 698 & 1210 \\
\hline \multirow{2}{*}{\multicolumn{2}{|c|}{$\begin{array}{l}\text { Partial } \\
\text { graph }\end{array}$}} & \multicolumn{3}{|c|}{20 commodities } & \multicolumn{3}{|c|}{56 commodities } & \multicolumn{3}{|c|}{90 commodities } \\
\hline & & $20 \mathrm{a}$ & $56 \mathrm{a}$ & $90 \mathrm{a}$ & $20 \mathrm{a}$ & $56 \mathrm{a}$ & $90 \mathrm{a}$ & $20 \mathrm{a}$ & $56 \mathrm{a}$ & $90 \mathrm{a}$ \\
\hline +SSPI & Time & 1.46 & 9.24 & 19.90 & 68.92 & 560.96 & 1027.86 & 487.53 & 3592.32 & 7791.59 \\
\hline \multirow[t]{2}{*}{ Str 2} & Cols & 567 & 1022 & 1420 & 2544 & 4459 & 5157 & 5333 & 86374 & 10978 \\
\hline & Iters & 86 & 106 & 109 & 266 & 317 & 295 & 444 & 433 & 429 \\
\hline (MP) & Time & 0.68 & 2.81 & 5.47 & 46.20 & 269.21 & 365.09 & 355.21 & 2211.86 & 4189.28 \\
\hline$(\mathrm{SP})$ & Time & 0.73 & 5.94 & 13.11 & 22.22 & 286.33 & 648.29 & 130.91 & 1366.63 & 3563.19 \\
\hline \multirow[t]{2}{*}{ (SSPI) } & NumIneq1 & 4 & 8 & 23 & 0 & 15 & 37 & 1 & 9 & 15 \\
\hline & NumIneq2 & 161 & 341 & 423 & 416 & 1151 & 1538 & 638 & 1639 & 2278 \\
\hline \multirow{2}{*}{\multicolumn{2}{|c|}{$\begin{array}{l}\text { Shioda et al. } \\
\text { instances }\end{array}$}} & \multicolumn{3}{|c|}{40 commodities } & \multicolumn{3}{|c|}{60 commodities } & \multicolumn{3}{|c|}{80 commodities } \\
\hline & & $20 \mathrm{a}$ & $40 \mathrm{a}$ & $60 \mathrm{a}$ & $20 \mathrm{a}$ & $40 \mathrm{a}$ & $60 \mathrm{a}$ & $20 \mathrm{a}$ & $40 \mathrm{a}$ & $60 \mathrm{a}$ \\
\hline +SSPI & Time & 20.65 & 14.16 & 14.66 & 164.17 & 112.81 & 129.75 & 1144.50 & 446.29 & 446.78 \\
\hline \multirow[t]{2}{*}{ Str 2} & Cols & 1946 & 1869 & 2101 & 4228 & 3675 & 4060 & 7725 & 6051 & 6193 \\
\hline & Iters & 181 & 84 & 56 & 349 & 162 & 118 & 598 & 251 & 163 \\
\hline (MP) & Time & 16.21 & 9.54 & 9.93 & 142.86 & 91.71 & 99.22 & 1076.00 & 394.54 & 386.17 \\
\hline$(\mathrm{SP})$ & Time & 4.33 & 4.39 & 4.42 & 21.01 & 20.54 & 29.40 & 67.94 & 50.84 & 58.95 \\
\hline \multirow[t]{2}{*}{ (SSPI) } & NumIneq1 & 0 & 0 & 0 & 0 & 0 & 0 & 0 & 5 & 0 \\
\hline & NumIneq2 & 222 & 161 & 117 & 348 & 308 & 327 & 422 & 318 & 328 \\
\hline \multirow{2}{*}{\multicolumn{2}{|c|}{$\begin{array}{l}\text { A1 } \\
\text { instances }\end{array}$}} & \multicolumn{3}{|c|}{21 commodities } & \multicolumn{3}{|c|}{55 commodities } & \multicolumn{3}{|c|}{91 commodities } \\
\hline & & $21 \mathrm{a}$ & $55 \mathrm{a}$ & $91 \mathrm{a}$ & $21 \mathrm{a}$ & 55 a & $91 \mathrm{a}$ & $21 \mathrm{a}$ & $55 \mathrm{a}$ & $91 \mathrm{a}$ \\
\hline+ SSPI & Time & 0.39 & 35.83 & 9.28 & 9.42 & 56.70 & 109.17 & 1921.80 & 1011.77 & 2217.27 \\
\hline \multirow[t]{2}{*}{ Str 2} & Cols & 188 & 422 & 497 & 565 & 765 & 1039 & 2549 & 2116 & 2222 \\
\hline & Iters & 39 & 54 & 36 & 120 & 89 & 67 & 467 & 264 & 175 \\
\hline (MP) & Time & 0.09 & 3.48 & 0.46 & 1.60 & 2.67 & 3.98 & 1361.62 & 69.15 & 70.63 \\
\hline$(\mathrm{SP})$ & Time & 0.28 & 32.06 & 8.31 & 7.53 & 52.33 & 101.45 & 558.87 & 934.88 & 2131.23 \\
\hline \multirow[t]{2}{*}{ (SSPI) } & NumIneq1 & 73 & 875 & 617 & 152 & 663 & 1263 & 298 & 1162 & 3004 \\
\hline & NumIneq2 & 56 & 114 & 67 & 158 & 216 & 208 & 418 & 663 & 581 \\
\hline
\end{tabular}

Table 4.18: Numerical results on a different separation strategy when adding SSPI to the column generation algorithm for the linear relaxation of (HPDW)

smaller than the corresponding $\mathrm{x}$-axis' value.

The results in Tables 4.19 and 4.20 and Figure 4.16 both show that adding (SSPI) reduces a lot the gap, for both (HPDW) and (HPL) formulations. As shown in Section 4.5. (HPDW) gap is smaller than (HPL) gap, and this is still valid adding (SSPI), apart for some partial graph and A1 instances for which solving (HPDW) was interrupted due to the time limit before all violated cuts are added. Using the second strategy for 
the cuts separation reduces the solution time and provides a bigger gap with respect to the first strategy, but we still have a big improvement compared to not adding (SSPI), for both formulations. Furthermore, for Shioda et al. instances we notice that (HPDW) formulation with (SSPI) and the second separation procedure has a smaller gap than (HPL) formulation with (SSPI) and the first separation procedure, and a smaller (or very similar) solution time. For complete and partial graph instances (HPDW) formulation with (SSPI) and the second separation procedure provides a gap which is of the same order of (HPL) formulation with (SSPI) and the first separation procedure, especially for instances with a lot of commodities and few toll paths, and the solution time is still smaller or very similar. For A1 instances the gap of both formulations with (SSPI) using both separation procedures is almost the same, but (HPL) formulation is much quicker to be solved. For this class of instances using (SSPI) provides a very small gap, less than one percent, and in some cases we are able to solve the integer problem at the root node.

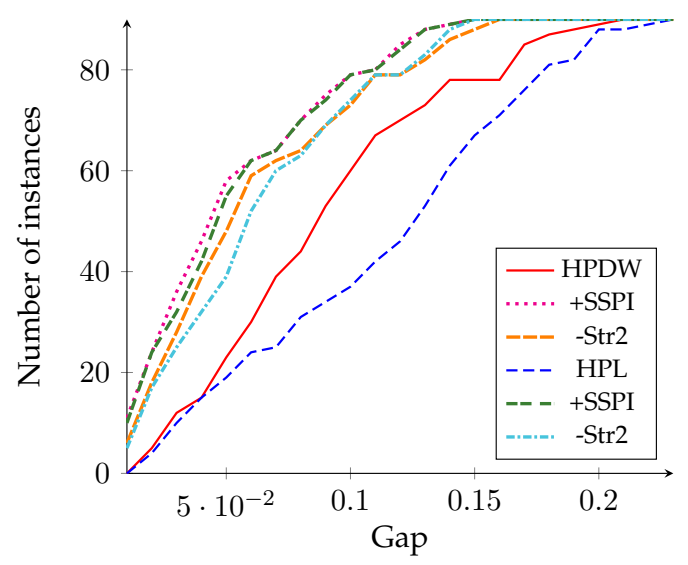

(a) Complete graph instances

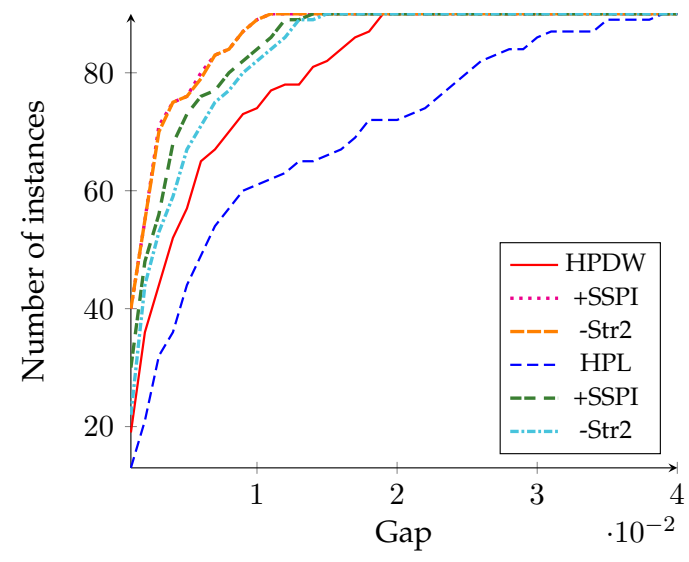

(c) Shioda et al.'s instances

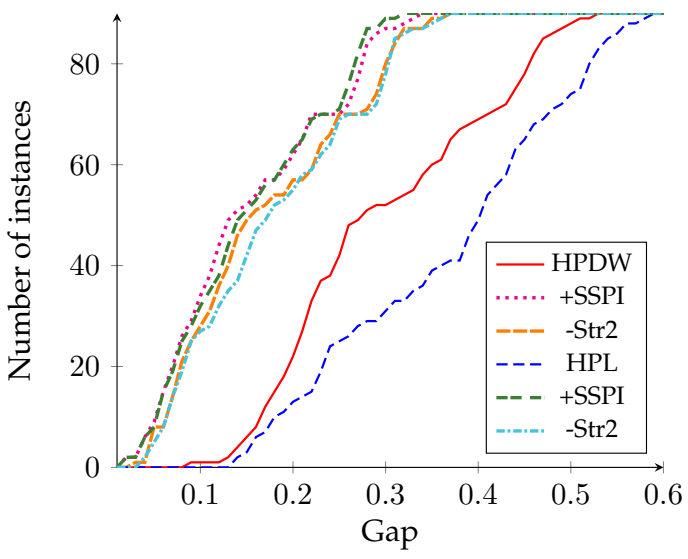

(b) Partial graph instances

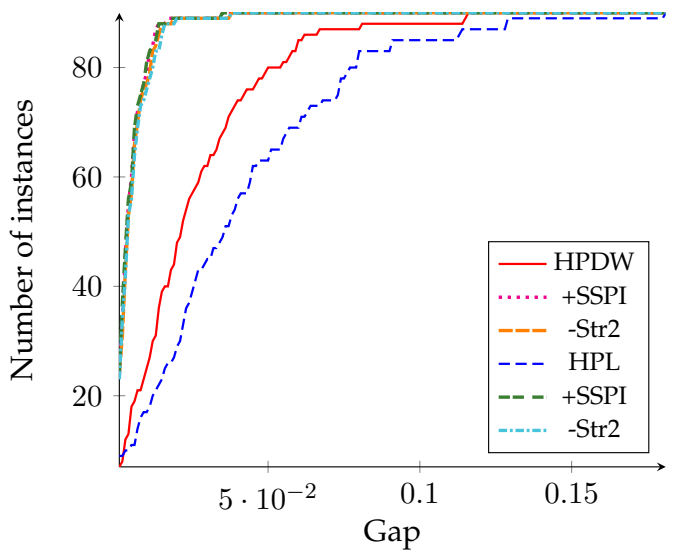

(d) A1 instances

Figure 4.16: Performance profile graphs on the gap for the linear relaxation for (HPL) and (HPDW) with SSPI 


\begin{tabular}{|c|c|c|c|c|c|c|c|c|c|c|}
\hline \multirow{2}{*}{\multicolumn{2}{|c|}{$\begin{array}{l}\text { Complete } \\
\text { graph }\end{array}$}} & \multicolumn{3}{|c|}{20 commodities } & \multicolumn{3}{|c|}{56 commodities } & \multicolumn{3}{|c|}{90 commodities } \\
\hline & & $20 \mathrm{a}$ & $56 \mathrm{a}$ & $90 \mathrm{a}$ & $20 \mathrm{a}$ & $56 a^{* 7}$ & $90 a^{* 10}$ & $20 \mathrm{a}$ & $56 a^{* 10}$ & $90 \mathrm{a}^{* 10}$ \\
\hline \multirow[t]{2}{*}{$(-\mathrm{L})$} & Gap & $6.19 \%$ & $3.71 \%$ & $3.15 \%$ & $12.82 \%$ & $11.18 \%$ & $11.51 \%$ & $15.33 \%$ & $16.88 \%$ & $17.47 \%$ \\
\hline & Time & 0.048 & 0.282 & 0.767 & 0.184 & 1.514 & 4.592 & 0.448 & 4.849 & 6.084 \\
\hline \multirow[t]{2}{*}{ +SSPI } & Gap & $1.83 \%$ & $1.25 \%$ & $0.88 \%$ & $3.94 \%$ & $5.43 \%$ & $6.63 \%$ & $4.51 \%$ & $9.7 \%$ & $11.14 \%$ \\
\hline & Time & 2.17 & 11.04 & 18.72 & 39.91 & 560.8 & 1054.21 & 242.82 & 3600.79 & 8217.28 \\
\hline \multirow[t]{2}{*}{ Str 2} & Gap & $2.36 \%$ & $1.91 \%$ & $1.51 \%$ & $4.92 \%$ & $6.39 \%$ & $7.75 \%$ & $6.05 \%$ & $10.9 \%$ & $12.27 \%$ \\
\hline & Time & 0.97 & 3.59 & 9.06 & 16.64 & 71.65 & 198.06 & 58.46 & 348.3 & 888.81 \\
\hline \multirow[t]{2}{*}{$(-\mathrm{DW})$} & Gap & $4.75 \%$ & $3.52 \%$ & $2.98 \%$ & $7.72 \%$ & $9.57 \%$ & $10.68 \%$ & $7.94 \%$ & $13.94 \%$ & $15.98 \%$ \\
\hline & Time & 0.199 & 0.726 & 1.57 & 4.13 & 12.7 & 22.3 & 25.84 & 62.26 & 108.99 \\
\hline \multirow[t]{2}{*}{ +SSPI } & Gap & $1.75 \%$ & $1.25 \%$ & $0.87 \%$ & $3.55 \%$ & $5.30 \%$ & $6.56 \%$ & $3.79 \%$ & $9.52 \%$ & $11.12 \%$ \\
\hline & Time & 1.80 & 9.28 & 12.90 & 260.58 & 1676.80 & 2470.51 & 2824.31 & 17801.94 & 18003.98 \\
\hline \multirow[t]{2}{*}{ Str 2} & Gap & $2.33 \%$ & $1.86 \%$ & $1.39 \%$ & $4.21 \%$ & $6.17 \%$ & $7.67 \%$ & $4.52 \%$ & $10.85 \%$ & $12.57 \%$ \\
\hline & Time & 0.70 & 1.92 & 4.59 & 35.51 & 105.03 & 188.44 & 225.14 & 470.59 & 1152.23 \\
\hline \multirow{2}{*}{\multicolumn{2}{|c|}{$\begin{array}{l}\text { Partial } \\
\text { graph }\end{array}$}} & \multicolumn{3}{|c|}{20 commodities } & \multicolumn{3}{|c|}{56 commodities } & \multicolumn{3}{|c|}{90 commodities } \\
\hline & & $20 \mathrm{a}$ & $56 \mathrm{a}$ & $90 \mathrm{a}$ & $20 \mathrm{a}$ & $56 a^{* 10}$ & $90 a^{* 10}$ & $20 a^{* 9}$ & $56 a^{* 10}$ & $90 a^{* 10}$ \\
\hline \multirow[t]{2}{*}{$(-\mathrm{L})$} & Gap & $22.78 \%$ & $20.49 \%$ & $19.58 \%$ & $36.37 \%$ & $41.91 \%$ & $40.69 \%$ & $42.87 \%$ & $48.67 \%$ & $52.06 \%$ \\
\hline & Time & 0.042 & 0.28 & 0.858 & 0.207 & 1.871 & 6.244 & 0.519 & 3.767 & 10.6 \\
\hline \multirow[t]{2}{*}{ +SSPI } & Gap & $5.88 \%$ & $6.61 \%$ & $6.11 \%$ & $11.2 \%$ & $19.52 \%$ & $20.34 \%$ & $13.57 \%$ & $23.19 \%$ & $27.18 \%$ \\
\hline & Time & 2.09 & 18.81 & 52.71 & 37.77 & 566.74 & 3170.62 & 180.84 & 3286.03 & 16865.64 \\
\hline \multirow[t]{2}{*}{ Str 2} & Gap & $6.9 \%$ & $7.8 \%$ & $7.33 \%$ & $13.38 \%$ & $22.29 \%$ & $23.51 \%$ & $16.29 \%$ & $26.77 \%$ & $31.97 \%$ \\
\hline & Time & 1.29 & 10.83 & 29.2 & 22.3 & 179.27 & 499.5 & 76.99 & 747.08 & 2106.4 \\
\hline \multirow[t]{2}{*}{$(-\mathrm{DW})$} & Gap & $17.64 \%$ & $19.30 \%$ & $19.03 \%$ & $22.19 \%$ & $36.33 \%$ & $37.67 \%$ & $23.95 \%$ & $39.84 \%$ & $46.52 \%$ \\
\hline & Time & 0.171 & 0.687 & 1.443 & 3.026 & 10.99 & 21.23 & 18.54 & 55.71 & 102.87 \\
\hline \multirow[t]{2}{*}{ +SSPI } & Gap & $5.65 \%$ & $6.55 \%$ & $6.08 \%$ & $10.57 \%$ & $19.39 \%$ & $20.33 \%^{* 1}$ & $12.34 \%$ & $23.60 \% * 5$ & $29.43 \% * 8$ \\
\hline & Time & 3.72 & 26.29 & 80.56 & 320.96 & 4028.78 & 11667.26 & 5645.97 & 18000 & 18000 \\
\hline \multirow[t]{2}{*}{ Str 2} & Gap & $6.53 \%$ & $7.72 \%$ & $7.41 \%$ & $11.86 \%$ & $21.98 \%$ & $23.36 \%$ & $13.77 \%$ & $26.06 \%$ & $31.62 \%$ \\
\hline & Time & 1.46 & 9.24 & 19.90 & 68.92 & 560.96 & 1027.86 & 487.53 & 3592.32 & 7791.59 \\
\hline
\end{tabular}

Table 4.19: Numerical results on linear relaxation for

(HPL) and (HPDW) formulations with SSPI -

Complete and partial graph instances 


\begin{tabular}{|c|c|c|c|c|c|c|c|c|c|c|}
\hline \multirow{2}{*}{\multicolumn{2}{|c|}{$\begin{array}{l}\text { Shioda et al. } \\
\text { instances }\end{array}$}} & \multicolumn{3}{|c|}{40 commodities } & \multicolumn{3}{|c|}{60 commodities } & \multicolumn{3}{|c|}{80 commodities } \\
\hline & & $20 \mathrm{a}$ & $40 \mathrm{a}$ & $60 \mathrm{a}$ & $20 \mathrm{a}$ & $40 \mathrm{a}$ & $60 \mathrm{a}$ & $20 a$ & $40 \mathrm{a}$ & $60 a$ \\
\hline \multirow[t]{2}{*}{$(-\mathrm{L})$} & Gap & $1.69 \%$ & $0.28 \%$ & $0.07 \%$ & $2.39 \%$ & $0.58 \%$ & $0.27 \%$ & $2.42 \%$ & $0.68 \%$ & $0.25 \%$ \\
\hline & Time & 0.09 & 0.21 & 0.48 & 0.13 & 0.39 & 0.78 & 0.18 & 0.55 & 1.16 \\
\hline \multirow[t]{2}{*}{ +SSPI } & Gap & $0.45 \%$ & $0.07 \%$ & $0.04 \%$ & $0.73 \%$ & $0.22 \%$ & $0.09 \%$ & $0.77 \%$ & $0.27 \%$ & $0.09 \%$ \\
\hline & Time & 33.7 & 43.09 & 11.36 & 109.93 & 138.28 & 350.02 & 230.67 & 362.27 & 530.76 \\
\hline \multirow[t]{2}{*}{ Str 2} & Gap & $0.54 \%$ & $0.1 \%$ & $0.06 \%$ & $0.8 \%$ & $0.27 \%$ & $0.12 \%$ & $0.88 \%$ & $0.32 \%$ & $0.12 \%$ \\
\hline & Time & 8.81 & 5.59 & 7.14 & 30.84 & 16.48 & 28.58 & 50.86 & 39.54 & 44.95 \\
\hline \multirow[t]{2}{*}{$(-\mathrm{DW})$} & Gap & $1.00 \%$ & $0.20 \%$ & $0.05 \%$ & $1.24 \%$ & $0.37 \%$ & $0.20 \%$ & $1.09 \%$ & $0.36 \%$ & $0.15 \%$ \\
\hline & Time & 5.51 & 7.79 & 11.39 & 43.12 & 47.04 & 56.83 & 198.38 & 221.28 & 260.09 \\
\hline \multirow[t]{2}{*}{ +SSPI } & Gap & $0.36 \%$ & $0.05 \%$ & $0.02 \%$ & $0.54 \%$ & $0.16 \%$ & $0.07 \%$ & $0.55 \%$ & $0.18 \%$ & $0.06 \%$ \\
\hline & Time & 30.31 & 25.93 & 19.78 & 436.25 & 492.85 & 551.81 & 6692.59 & 1836.38 & 2805.57 \\
\hline \multirow[t]{2}{*}{ Str 2} & Gap & $0.36 \%$ & $0.06 \%$ & $0.02 \%$ & $0.54 \%$ & $0.17 \%$ & $0.07 \%$ & $0.55 \%$ & $0.19 \%$ & $0.07 \%$ \\
\hline & Time & 20.65 & 14.16 & 14.66 & 164.17 & 112.81 & 129.75 & 1144.50 & 446.29 & 446.78 \\
\hline \multirow{2}{*}{\multicolumn{2}{|c|}{$\begin{array}{l}\text { A1 } \\
\text { instances }\end{array}$}} & \multicolumn{3}{|c|}{21 commodities } & \multicolumn{3}{|c|}{55 commodities } & \multicolumn{3}{|c|}{91 commodities } \\
\hline & & $21 \mathrm{a}$ & $55 \mathrm{a}$ & $91 \mathrm{a}$ & $21 \mathrm{a}$ & $55 \mathrm{a}$ & $91 \mathrm{a}$ & $21 \mathrm{a}$ & $55 \mathrm{a}$ & $91 \mathrm{a}$ \\
\hline \multirow[t]{2}{*}{$(-\mathrm{L})$} & Gap & $5.30 \%$ & $2.78 \%$ & $1.41 \%$ & $6.36 \%$ & $3.57 \%$ & $1.74 \%$ & $6.26 \%$ & $4.34 \%$ & $3.12 \%$ \\
\hline & Time & 0.13 & 0.48 & 1.21 & 0.50 & 2.36 & 5.70 & 1.34 & 5.83 & 14.96 \\
\hline \multirow[t]{2}{*}{ +SSPI } & Gap & $0.31 \%$ & $0.4 \%$ & $0.19 \%$ & $0.49 \%$ & $0.52 \%$ & $0.21 \%$ & $0.6 \%$ & $0.7 \%$ & $0.43 \%$ \\
\hline & Time & 0.31 & 10.79 & 4.98 & 2.32 & 14.02 & 32.52 & 55.08 & 167.31 & 400.36 \\
\hline \multirow[t]{2}{*}{ Str 2} & Gap & $0.32 \%$ & $0.44 \%$ & $0.21 \%$ & $0.64 \%$ & $0.54 \%$ & $0.22 \%$ & $0.72 \%$ & $0.78 \%$ & $0.57 \%$ \\
\hline & Time & 0.28 & 6.03 & 4.3 & 2.26 & 13.77 & 27.05 & 17.28 & 70.31 & $146.83 \%$ \\
\hline \multirow[t]{2}{*}{$(-\mathrm{DW})$} & Gap & $3.80 \%$ & $2.30 \%$ & $1.35 \%$ & $3.14 \%$ & $2.52 \%$ & $1.25 \%$ & $2.41 \%$ & $2.97 \%$ & $2.18 \%$ \\
\hline & Time & 0.07 & 0.22 & 0.46 & 0.48 & 1.69 & 3.85 & 3.14 & 8.63 & 16.45 \\
\hline \multirow[t]{2}{*}{ + SSPI } & Gap & $0.31 \%$ & $0.43 \%$ & $0.22 \%$ & $0.48 \%$ & $0.52 \%$ & $0.22 \%$ & $0.61 \%$ & $0.71 \%$ & $0.44 \%$ \\
\hline & Time & 0.50 & 87.02 & 12.85 & 18.83 & 107.15 & 213.77 & $2249.15^{* 1}$ & $3852.73^{* 1}$ & $9867.31^{* 3}$ \\
\hline \multirow[t]{2}{*}{ Str 2} & Gap & $0.32 \%$ & $0.46 \%$ & $0.21 \%$ & $0.49 \%$ & $0.54 \%$ & $0.23 \%$ & $0.63 \%$ & $0.76 \%$ & $0.55 \%$ \\
\hline & Time & 0.39 & 35.83 & 9.28 & 9.42 & 56.70 & 109.17 & 1921.80 & 1011.77 & 2217.27 \\
\hline
\end{tabular}

Table 4.20: Numerical results on linear relaxation for

(HPL) and (HPDW) formulations with SSPI -

Shioda et al. and A1 instances 


\section{Chapter 5}

\section{Branch-(and-Cut)-and-Price framework for the NPP with Connected Toll Arcs}

\subsection{Introduction}

This chapter presents the Branch-(and-Cut)-and-Price algorithm that we developed to solve the Dantzig-Wolfe reformulation of the NPP with connected toll arcs or (HP) that we presented in Chapter 4 In Chapter 4 we discussed the column generation algorithm to solve its linear relaxation, presenting several improvement strategies for it. Now, in this chapter, we approach the problem of solving the integer formulation.

First, given its parallel to the product pricing problem, we consider results from the literature for the product pricing problem, study them into more details and use them to provide further insights on the (HP). We consider its inverse problem, searching for optimal tolls once commodities have been already assigned to paths, and we show that it is polynomial. Then, using this inverse problem, we develop some heuristics to find good integer solutions for the (HP).

Afterwards we present different branching rules to construct the tree, detailing the Branch-and-Price algorithm. We also integrate the heuristic algorithms presented in the first part of the chapter, and we add the valid inequalities that we reformulated in the last section of Chapter 4 , leading to a Branch-and-Cut-and-Price algorithm. All the proposed strategies are tested on our instances sets and we present numerical results for them.

\subsection{The price setting subproblem}

In Shioda et al. (2011), authors consider a product pricing problem, in which a company maximises the revenue from a set of products sold on the market and the customer 
choice behaviour is based on reservation prices, i.e. the maximum price each customer is willing to pay for each product. Each customer buys the product providing the maximum utility, which is the difference between the reservation price and the price of the product. We introduced this problem in Section 1.6, reporting results from Heilporn et al. (2010a), where the parallel between this problem and the (HP) is shown.

Shioda et al. (2011) propose the following mixed integer non linear formulation for the product pricing problem:

$$
\begin{array}{llr}
\max _{\pi, x} & \sum_{k \in \mathcal{K}} \sum_{j \in \mathcal{J}} \eta^{k} \pi_{j} x_{j}^{k}, & \\
\text { s.t. } & \sum_{i \in \mathcal{J}: i \neq j}\left(r_{i}^{k}-\pi_{i}\right) x_{i}^{k} \geq r_{j}^{k} \sum_{i \in \mathcal{J}: i \neq j} x_{i}^{k}-\pi_{j} & \forall k \in \mathcal{K}, \forall j \in \mathcal{J}, \\
& \left(r_{j}^{k}-\pi_{j}\right) x_{j}^{k} \geq 0 & \forall k \in \mathcal{K}, \forall j \in \mathcal{J}, \\
& \sum_{j \in \mathcal{J}} x_{j}^{k} \leq 1 & \forall k \in \mathcal{K}, \\
& \pi_{j} \geq 0 & \forall j \in \mathcal{J}, \\
& x_{j}^{k} \in\{0,1\} & \forall k \in \mathcal{K}, \forall j \in \mathcal{J} .
\end{array}
$$

where variables $x_{j}^{k}$ define the buying choice of customer $k$ for product $j$, variables $\pi_{j}$ the price of product $j$ and parameters $r_{j}^{k}$ the reservation price of customer $k$ for product $j$. Similarly as the model by Heilporn et al. (2010a) presented in Section 1.6, constraints (5.1b) ensure that the product bought by each customer has the maximal utility, constraints (5.1c) guarantee that a customer is only buying a product with a positive utility and constraints (5.1d) allow each customer to buy at most one product. The objective function maximises the company's revenue from the products bought by customers.

Starting from this problem, Shioda et al. (2011) present the Price Setting Subproblem (PSS), which is the problem of finding the optimal prices for products, once the customers' choices of which product to buy are given. Variables $x_{j}^{k}$ are fixed to 1 if the customer $k$ is buying product $j, 0$ otherwise. In the (PSS) the company is maximising its revenue, and prices are set to ensure that commodities buying choices maximise their utility.

Denote $\mathcal{K}_{j}$ as the set of customers buying product $j$ :

$$
\mathcal{K}_{j}=\left\{k \in \mathcal{K}: x_{j}^{k}=1\right\}
$$

$\eta_{j}$ as the total demand of customers buying product $j$ :

$$
\eta_{j}=\sum_{k \in \mathcal{K}_{j}} \eta^{k},
$$


and $\mathcal{B}$ as the set of products that are bought by at least one customer:

$$
\mathcal{B}=\left\{j: \mathcal{K}_{j} \neq \emptyset\right\}
$$

Simplifying from the product pricing problem formulation defined by Equations (5.1a) to (5.1f), the Price Setting Subproblem (PSS) can be written as follows:

$$
\begin{aligned}
& \text { (PSS) } \\
& \max _{\pi} \sum_{j \in \mathcal{B}} \eta_{j} \pi_{j} \\
& \text { s.t. } \quad r_{j}^{k}-\pi_{j} \geq r_{i}^{k}-\pi_{i} \quad \forall j \in \mathcal{B}, \forall k \in \mathcal{K}_{j}, \forall i \in \mathcal{J}: i \neq j, \\
& r_{j}^{k}-\pi_{j} \geq 0 \quad \forall j \in \mathcal{B}, \forall k \in \mathcal{K}_{j}, \\
& \pi_{j} \geq 0
\end{aligned}
$$

Let us introduce some more parameters. Define:

$$
\overline{r_{j i}}=\min _{k \in \mathcal{K}_{j}}\left\{r_{j}^{k}-r_{i}^{k}\right\} \quad \forall j \in \mathcal{B}, \forall i \in \mathcal{J}: i \neq j
$$

and

$$
\overline{r_{j}}=\min _{k \in \mathcal{K}_{j}}\left\{r_{j}^{k}\right\} \quad \forall j \in \mathcal{B} .
$$

Therefore, using these parameters, the problem can be simplified as follows:

$$
\begin{aligned}
& \text { (PSS) } \\
& \max _{\pi} \sum_{j \in \mathcal{B}} \eta_{j} \pi_{j}, \\
& \text { s.t. } \quad \pi_{j}-\pi_{i} \leq \overline{r_{j i}} \\
& \forall j \in \mathcal{B}, \forall i \in \mathcal{J}: i \neq j, \\
& \pi_{j} \leq \overline{r_{j}} \\
& \forall j \in \mathcal{B} \text {, } \\
& \pi_{j} \geq 0
\end{aligned}
$$

We have to be careful because (PSS) may be unfeasible $]^{1}$ First, if there exists a directed cycle $\mathcal{C}$ such that $\sum_{(i, j) \in \mathcal{C}} \overline{r_{i j}}<0$, then summing constraints $5.8 \mathrm{~b}$ for all $(i, j) \in \mathcal{C}$ yields to $0 \leq \sum_{(i, j) \in \mathcal{C}} \overline{r_{i j}}(<0)$, which is impossible. Further, even if all cycles have positive costs, there may be no solution satisfying constraints (5.8b) and (5.8c) such that all $\pi_{j}$ are non negative. Therefore, we keep constraints (5.8d) in the (PSS) formulation, contrary to the formulation used by Shioda et al. (2011).

Fortunately, infeasibility of (PSS) can be detected using its dual, denoted as (D-PSS): if (D-PSS) is feasible and unbounded, then (PSS) is unfeasible.

\footnotetext{
${ }^{1}$ This possibility is not considered by Shioda et al. (2011).
} 
Let us consider the dual of (PSS) composed by Equations (5.8a) to (5.8d). It can be written as follows:

$$
\begin{aligned}
& \text { (D-PSS) } \\
& \min _{\phi, \omega} \sum_{j \in \mathcal{J}} \sum_{i \in \mathcal{J}: i \neq j} \overline{r_{j i}} \phi_{j i}+\sum_{j \in \mathcal{J}} \overline{r_{j}} \omega_{j}, \\
& \text { s.t. } \quad \sum_{i \in \mathcal{J}: i \neq j} \phi_{j i}-\sum_{i \in \mathcal{J}: i \neq j} \phi_{i j}+\omega_{j} \geq \eta_{j} \\
& -\sum_{i \in \mathcal{B}: i \neq j} \phi_{i j} \geq 0 \\
& \forall j \in \mathcal{B}, \\
& \forall j \notin \mathcal{B}, \\
& \phi_{j i} \geq 0 \\
& \forall j \in \mathcal{B}, \forall i \in \mathcal{J}: i \neq j, \\
& \omega_{j} \geq 0
\end{aligned}
$$

where dual variables $\phi_{j i}$ are associated to constraints (5.8b), for each $j \in \mathcal{B}$ and each $i \in \mathcal{J}: i \neq j$, and variables $\omega_{j}$ to constraints (5.8c), for each $j \in \mathcal{B}$.

One can easily notice that non negativity constraints $(5.9 \mathrm{~d})$ and constraints $(5.9 \mathrm{c})$ implies that $\phi_{i j}=0$ for all $i \in \mathcal{B}$ and $j \notin \mathcal{B}$. Therefore we can remove from (D-PSS) all variables $\phi_{i j}, i \in \mathcal{B}$ and $j \notin \mathcal{B}$. Furthermore, by adding a single redundant constraint (5.10c) which is the sum of constraints (5.9b), (D-PSS ) has the following formulation:

(D-PSS)

$$
\begin{array}{lll}
\min _{\phi, \omega} & \sum_{j \in \mathcal{B}} \sum_{i \in \mathcal{B}: i \neq j} \bar{r}_{j i} \phi_{j i}+\sum_{j \in \mathcal{J}} \overline{r_{j}} \omega_{j}, & \\
\text { s.t. } & \sum_{i \in \mathcal{B}: i \neq j} \phi_{j i}-\sum_{i \in \mathcal{B}: i \neq j} \phi_{i j}+\omega_{j} \geq \eta_{j} & \\
& \sum_{j \in \mathcal{B}} \omega_{j} \geq \sum_{j \in \mathcal{B}} \eta_{j} & \\
& \phi_{j i} \geq 0 & \forall j \in \mathcal{B}, \\
& \omega_{j} \geq 0 & \forall j \in \mathcal{B}, \forall i \in \mathcal{B}: i \neq j, \\
& \forall j \in \mathcal{B} .
\end{array}
$$

Shioda et al. (2011) show that their (D-PSS) formulation, which differs from our formulation by transforming constraints (5.10b) and (5.10c) into equalities, corresponds to a formulation of $|\mathcal{B}|$ shortest path problems.

The interpretation of our (D-PSS) formulation, described by Equations (5.10a) to (5.10e), is similar and is the following. Consider the graph presented in Figure 5.1 . where there is a node for each product $j \in \mathcal{B}$ and an extra sink node $s$. There is an arc connecting each pair of nodes $j, i$ of $\mathcal{B}$, of cost $\overline{r_{j i}}$, and an arc connecting each node $j$ of $\mathcal{B}$ to node $s$, of cost $\overline{r_{j}}$. Variables $\phi_{j i}$ represent the flow going from node $j$ to node $i$ and variables $\omega_{j}$ from node $j$ to the sink $s$. Constraints (5.10b) ensure that a positive flow 
of at least $\eta_{j}$ units exits from each node $j \in \mathcal{B}$, which enters in the sink $s$ by constraint (5.10c). As there are no capacities on the network, each flow from $j$ to $s$ follows a shortest path. Let us remark that, being a flow problem on a connected graph without capacities, (D-PSS) is always feasible. The optimal solution of the (PSS), i.e. the price of each product $j$, is equal to the length of this shortest path from $j$ to $s$.

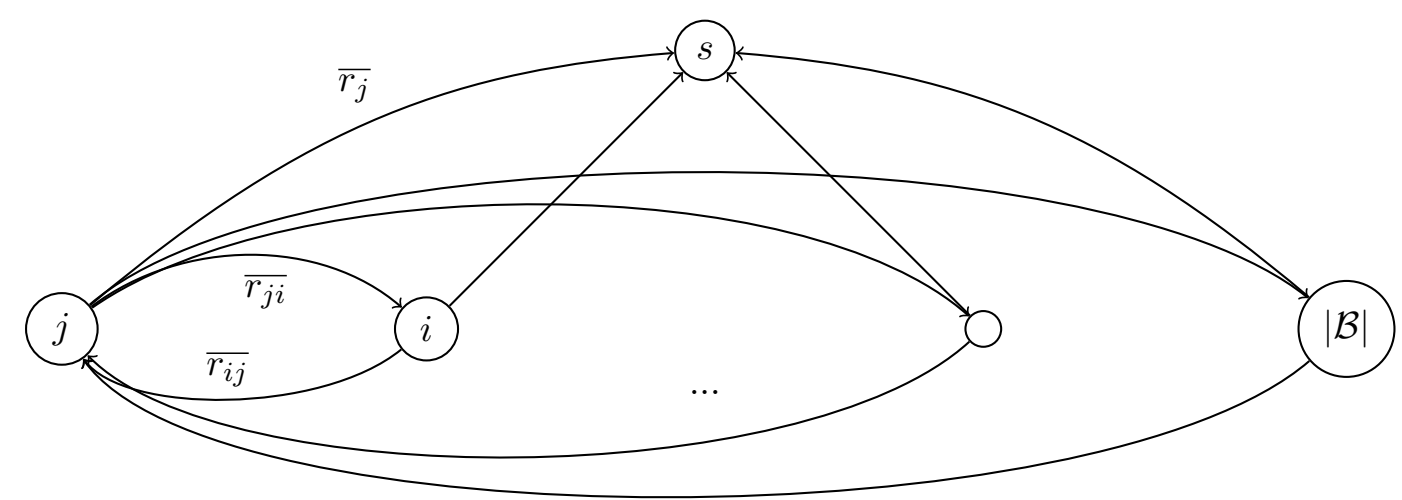

Figure 5.1: Flow network for (D-PSS) formulation

We can therefore identify the following conditions for the feasibility of (PSS):

(i) If there exists a cycle $\mathcal{C}$ with negative cost, then the dual problem is unbounded since one can construct a solution with unbounded positive values for the variables $\phi_{i j}$ such that $(i, j) \in \mathcal{C}$. Consequently, (PSS) is unfeasible.

(ii) If there is no negative cost cycle but there exists a node $j$ for which the shortest path to the sink $s$ has a negative cost, then the dual problem is again unbounded because one could set the variables $\phi_{i j}$, belonging to that path, to unbounded positive values and decrease the objective function value. This corresponds to the case where there are no $\pi_{j} \geq 0$ values satisfying constraints (5.8b) and (5.8c), i.e. (PSS) is unfeasible.

(iii) When there is no negative cost cycle and the shortest paths from all nodes $j \in \mathcal{B}$ to the sink $s$ have non negative costs, then both primal and dual problems are feasible and bounded.

Furthermore, let us underline that (D-PSS) and (PSS) are polynomially solvable, being linear problems, and that, if they are feasible and bounded and all parameters are integer, then their optimal solutions are integral. 


\subsection{The Inverse Problem for the NPP with connected toll arcs}

Consider now the NPP with connected toll arcs or (HP) and its single level non linear formulation (HPNL) presented in Section 1.5.6, which is as follows:

$$
\begin{array}{rrr}
\text { (HPNL) } & \\
\max _{T, x} & \sum_{a \in \mathcal{A}} \sum_{k \in \mathcal{K}} \eta^{k} T_{a} x_{a}^{k}, & \\
\text { s.t. } & \sum_{a \in \mathcal{A}}\left(T_{a}-M_{a}^{k}\right) x_{a}^{k}-T_{b} \leq-M_{b}^{k} & \forall k \in \mathcal{K}, \forall b \in \mathcal{A}, \\
& \sum_{a \in \mathcal{A}}\left(T_{a}-M_{a}^{k}\right) x_{a}^{k} \leq 0 & \forall k \in \mathcal{K}, \\
& \sum_{a \in \mathcal{A}} x_{a}^{k} \leq 1 & \forall k \in \mathcal{K}, \\
& T_{a} \geq 0 & \forall a \in \mathcal{A}, \\
& x_{a}^{k} \in\{0,1\} & \forall k \in \mathcal{K}, \forall a \in \mathcal{A} .
\end{array}
$$

Let us remind the parallel between the (HP) and the product pricing problem revealed by Heilporn et al. (2010a) and reported in Section 1.6 paths $a$ correspond to products $j$, tolls $T_{a}$ to prices $\pi_{j}$ and toll windows $M_{a}^{k}$ to reservation prices $r_{j}^{k}$. We can notice that this (HP) formulation is almost equal to the product price non linear formulation (Equations (5.1a) to (5.1f) ) presented in the previous section. There are two differences: in constraints $\sqrt{5.11 b}$ ) the term $M_{b}^{k}$ is not multiplied by the sum of assignment variables $x_{a}^{k}$ as in constraints $5.5 \mathrm{~b}$ ) for the term $r_{j}^{k}$, and constraints $5.11 \mathrm{c}$ ) correspond to the sum over products $j$ of constraints $5.5 c$. . However, when fixing assignment variables $x_{a}^{k}$, i.e. considering the equivalent of the (PSS) for our (HP), we will see that we have similar results.

Given a fixed assignment of commodities to paths, we define the Inverse Problem (IP) for the NPP with connected toll arcs or (HP) as the problem of finding optimal tolls such that the shortest path for each commodity is the one to which it is assigned. This means that flow variables $x_{a}^{k}$ have been fixed to either 0 or 1 , for each $a \in \mathcal{A}$ and $k \in \mathcal{K}$, defining an assignment matrix. We only consider assignment matrices in which each commodity $k$ has been assigned to at maximum one toll path $a$, if not the problem will be unfeasible (constraints (5.11d)). The objective function of (IP) is the same as for (HP), i.e. maximising the leader's revenue. The constraints of (IP) must assure that the given assignment is optimal, such that paths with flow variables equal to 1 for a subset of commodities are the most attractive for this subset of commodities. It may happen that there does not exist any combination of tolls providing the given assignment: in this case the (IP) is unfeasible. 
Let us define a set $\mathcal{K}_{a}$ of commodities that have been assigned to path $a$, for each $a \in \mathcal{A}$ :

$$
\mathcal{K}_{a}=\left\{k \in \mathcal{K}: x_{a}^{k}=1\right\}
$$

and a set $\mathcal{K}_{o d}$ of commodities that have been assigned to their toll free path:

$$
\mathcal{K}_{\text {od }}=\left\{k \in \mathcal{K}: x_{\text {od }}^{k}=1\right\}
$$

where $x_{o d}^{k}=1-\sum_{a \in \mathcal{A}} x_{a}^{k}$, for each $k \in \mathcal{K}$. One can see that $\mathcal{K}_{o d}=\mathcal{K} \backslash\left(\bigcup_{a \in \mathcal{A}} \mathcal{K}_{a}\right)$.

We also define a set $\mathcal{U}$ as the set of toll paths that are chosen by at least one commodity:

$$
\mathcal{U}=\left\{a \in \mathcal{A}: \mathcal{K}_{a} \neq \emptyset\right\}
$$

Finally, we define a cumulative demand for each toll path $a \in \mathcal{A}$, as the sum of the demand of all commodities assigned to this path:

$$
\eta_{a}=\sum_{k \in \mathcal{K}_{a}} \eta^{k}
$$

Therefore, simplifying from (HPNL) formulation represented by Equations (5.11a) to $5.11 \mathrm{f}$, and similarly as done in the previous section for the (PSS), the (IP) can be formulated as follows:

(IP)

$$
\begin{aligned}
& \max _{T} \sum_{a \in \mathcal{U}} \eta_{a} T_{a} \\
& \text { s.t. } \quad T_{a}-M_{a}^{k}-T_{b} \leq-M_{b}^{k} \quad \forall a \in \mathcal{U}, \forall k \in \mathcal{K}_{a}, \forall b \in \mathcal{A}: b \neq a, \\
& -T_{b} \leq-M_{b}^{k} \quad \forall b \in \mathcal{A}, \forall k \in \mathcal{K}_{o d}, \\
& T_{a}-M_{a}^{k} \leq 0 \quad \forall a \in \mathcal{U}, \forall k \in \mathcal{K}_{a}, \\
& T_{a} \geq 0 \quad \forall a \in \mathcal{A} \text {. }
\end{aligned}
$$

To further simplify the formulation we introduce the following parameters:

- $\overline{M_{a}}=\min _{k \in \mathcal{K}_{a}}\left\{M_{a}^{k}\right\}$, for each $a \in \mathcal{U}$;

- $\underline{M_{a}}=\max _{k \in \mathcal{K}_{o d}}\left\{M_{a}^{k}\right\}$, for each $a \in \mathcal{A}$;

- $\overline{M_{a b}}=\min _{k \in \mathcal{K}_{a}}\left\{M_{a}^{k}-M_{b}^{k}\right\}$, for each $a \in \mathcal{U}$ and $b \in \mathcal{A}$ such that $b \neq a$. 
Using these parameters, (IP) can be rewritten as follows:

(IP)

$$
\begin{array}{llr}
\max _{T} & \sum_{a \in \mathcal{U}} \eta_{a} T_{a}, & \\
\text { s.t. } & T_{a}-T_{b} \leq \overline{M_{a b}} & \forall a \in \mathcal{U}, \forall b \in \mathcal{A}: b \neq a, \\
& T_{a} \leq \overline{M_{a}} & \forall a \in \mathcal{U}, \\
& T_{a} \geq \underline{M_{a}} & \forall a \in \mathcal{A}, \\
& T_{a} \geq 0 & \forall a \in \mathcal{A} .
\end{array}
$$

Assume $\underline{M_{a}} \leq \overline{M_{a}}$, for all $a \in \mathcal{U}$ : otherwise the problem is not feasible. Then, define a new set of variables, for all $a \in \mathcal{A}$ :

$$
T_{a}^{\prime}=T_{a}-\underline{M_{a}} .
$$

Define also new parameters as follows:

- $M_{a}^{\prime}=\overline{M_{a}}-\underline{M_{a}}$, for each $a \in \mathcal{U}$;

- $M_{a b}^{\prime}=\overline{M_{a b}}-\underline{M_{a}}+\underline{M_{b}}$, for each $a \in \mathcal{U}$ and $b \in \mathcal{A}$ such that $b \neq a$.

The problem becomes:

(IP)

$$
\begin{array}{llr}
\max _{T^{\prime}} & \sum_{a \in \mathcal{U}} \eta_{a} T_{a}^{\prime}+\sum_{a \in \mathcal{U}} \underline{M_{a}} \eta_{a}, & \\
\text { s.t. } & T_{a}^{\prime}-T_{b}^{\prime} \leq M_{a b}^{\prime} & \forall a \in \mathcal{U}, \forall b \in \mathcal{A}: b \neq a, \\
& T_{a}^{\prime} \leq M_{a}^{\prime} & \forall a \in \mathcal{U}, \\
& T_{a}^{\prime} \geq 0 & \forall a \in \mathcal{A} .
\end{array}
$$

where the second term of the objective function is a constant, and it can be removed without changing the optimal solution, but we have to keep it to obtain an optimal solution value equal to that of the original problem.

Therefore the resulting (IP) formulation represented by Equations (5.19a) to 5.19d) corresponds to the formulation of the (PSS) represented by Equations (5.8a) to (5.8d) and we can formulate its dual in the same way, as follows: 
(D-IP)

$$
\begin{array}{lll}
\min _{\phi, \omega} & \sum_{a \in \mathcal{U}} \sum_{b \in \mathcal{U}: b \neq a} M_{a b}^{\prime} \phi_{a b}+\sum_{a \in \mathcal{U}} M_{a}^{\prime} \omega_{a}, & \\
\text { s.t. } & \sum_{b \in \mathcal{U}: b \neq a} \phi_{a b}-\sum_{b \in \mathcal{U}: b \neq a} \phi_{b a}+\omega_{a} \geq \eta_{a} & \forall a \in \mathcal{U}, \\
& \phi_{a b} \geq 0 & \forall a \in \mathcal{U}, \forall b \in \mathcal{U}: b \neq a, \\
& \omega_{a} \geq 0 & \forall a \in \mathcal{U},
\end{array}
$$

where dual variables $\phi_{a b}$ are associated to constraints (5.19b) and $\omega_{a}$ to constraints (5.19c) of (IP). The interpretation of (D-IP) formulation as a non capacitated flow problem and the conditions for the feasibility of its primal problem are the same as for the (D-PSS) formulation, so we do not repeat them.

We now use the properties of (IP) and (D-IP) to derive some results on the (HP).

Proposition 5.3.1 (Integrality of (IP)). If all parameters are integer, then the (IP) formulation composed by Equations 5.19a) to 5.19d) is integral.

Proof. The matrix associated to the constraints of the formulation is totally unimodular, which implies the result.

Proposition 5.3.2 ((HPNL) is a full integer formulation). If all parameters are integer, then the (HPNL) formulation composed by Equations (5.11a) to (5.11f) is integral.

Proof. The integrality property of (IP) holds for every assignment matrix we choose, and in particular for the optimal assignment matrix that we obtain when solving (HPNL) formulation.

Therefore in the (HPNL) formulation (Equations (5.11a) to (5.11f) ) we could replace constraints 5.11e $T_{a} \geq 0$ with $T_{a} \in \mathbb{N}$, for each $a \in \mathcal{A}$, leading to a full integer formulation.

\subsection{Heuristics for the NPP with connected toll arcs}

In this section we propose two heuristic algorithms for the NPP with connected toll arcs or (HP). Together with the column generation algorithm that we presented in Chapter 4 these heuristics will be integrated in the Branch-and-Price algorithm that we present in the second part of the chapter, aiming to find good integer solutions in the nodes of the tree. Therefore details on how to integrate them in the Branch-and-Price algorithm and numerical results on their performance will be presented after we introduce the branching strategies, in Sections 5.7 and 5.8.3 respectively. 
These heuristic algorithms start from a fractional solution of the linear relaxation of the (HPNL) formulation and construct a feasible integer solution. Consider a fractional solution of the linear relaxation of the (HPNL) formulation, provided for instance by solving the Dantzig-Wolfe reformulation with the column generation algorithm presented in Chapter 4. Let us denote the fractional values of the variables for this solution as $\widetilde{T_{a}}, \widetilde{x_{a}^{k}}$ and $\widetilde{p_{a}^{k}}$, for each $a \in \mathcal{A}$ and $k \in \mathcal{K}$. We now propose two heuristic algorithms that attempt to construct a feasible integer solution.

\subsubsection{Heuristic 1}

In this first heuristic algorithm we start from the fractional toll values $\widetilde{T_{a}}$ provided by solving the linear relaxation of (HPNL) and we construct a feasible integer solution starting from it, with the following algorithm.

Algorithm 5.4.1 (Heuristic 1 - (H1)).

Step 1: for each toll path $a \in \mathcal{A}$, set the toll equal to the maximum between the fractional value, rounded down, and the minimum $M_{a}^{k}$, for all $k \in \mathcal{K}$, as follows:

$$
T_{a}=\max \left\{\left\lfloor\widetilde{T}_{a}\right\rfloor, \min _{k \in \mathcal{K}} M_{a}^{k}\right\} \quad \forall a \in \mathcal{A} .
$$

We round down the fractional values to have integer toll values to avoid numerical problems in the column generation algorithm, and we set the tolls equal to at least the smallest $M_{a}^{k}$ as we noticed that it improves the solution.

Step 2: find the assignment of commodities to toll paths ( $x_{a}^{k}$ values) when applying these tolls, searching for the shortest path for each commodity. Calculate the assignment matrix as follows, for all $k \in \mathcal{K}$ :

- if $\min _{a \in \mathcal{A}}\left\{c_{a}^{k}+T_{a}\right\} \leq c_{o d}^{k}$ then

$$
x_{\bar{a}}^{k}=1 \quad \text { where } \quad \bar{a}=\arg \min _{a \in \mathcal{A}}\left\{c_{a}^{k}+T_{a}\right\}
$$

and $x_{a}^{k}=0$, for all $a \neq \bar{a}$,

- otherwise $x_{a}^{k}=0$ for all $a \in \mathcal{A}$.

Step 3: calculate the leader's revenue corresponding to this solution:

$$
\mathcal{R}(H 1)=\sum_{a \in \mathcal{A}} \sum_{k \in \mathcal{K}} \eta^{k} T_{a} x_{a}^{k}
$$

\subsubsection{Heuristic 2}

In this second heuristic we start from the fractional assignment values $\widetilde{x}_{a}^{k}$ provided by solving the linear relaxation of (HPNL) and we try to construct a feasible integer solution using the Inverse Problem, with the following algorithm. 
Algorithm 5.4.2 (Heuristic 2 - (H2)).

Step 1: construct an integer assignment matrix rounding the fractional one, as follows:

$$
x_{a}^{k}=\left\{\begin{array}{ll}
0 & \text { if } \widetilde{x}_{a}^{k}<0.5 \\
1 & \text { otherwise }
\end{array} \quad \forall a \in \mathcal{A}, \forall k \in \mathcal{K} .\right.
$$

It may happen that in the fractional solution there are one commodity $k$ and two different paths $a, b$ such that $\widetilde{x}_{a}^{k}=\widetilde{x}_{b}^{k}=0.5$. As we want to have a feasible assignment matrix, i.e. each commodity is assigned to at most one toll path, we assign the commodity to the first path in the list, choosing $x_{a}^{k}=1$ and $x_{b}^{k}=0$.

Step 2: use this assignment matrix to solve the Inverse Problem detailed in Section 5.3 . It may happen that the (IP) is not feasible, meaning that there does not exist a set of tolls providing this assignment matrix. If it is the case we stop. If the (IP) is feasible, its optimal solution provides a set of integer tolls $T_{a}$ for each $a \in \mathcal{A}$.

Step 3: calculate the leader's revenue corresponding to this solution:

$$
\mathcal{R}(H 2)=\sum_{a \in \mathcal{A}} \sum_{k \in \mathcal{K}} \eta^{k} T_{a} x_{a}^{k}
$$

Note that it is possible to improve the solution iterating between these heuristics, applying Heuristic 1 (resp. 2) to the solution of Heuristic 2 (resp. 1). Furthermore, note that whilst Heuristic 1 is always able to construct a feasible integer solution, this is not true for Heuristic 2. However, if Heuristic 2 is applied starting from the solution of Heuristic 1, then it will surely provide a feasible solution, as the toll values used in Heuristic 1 represent a feasible solution.

\subsection{Branching rules}

To solve the Dantzig-Wolfe reformulation of the (HP) that we presented in Chapter 4 , Section 4.3 we use a Branch-and-Bound framework (see for instance Wolsey, 1998, chap. 7). We create a branching tree and in each node of the tree we solve the associated linear problem with the column generation algorithm 4.3.1 presented in Section 4.3.1. In this section we propose different branching rules to create the nodes of the tree.

First of all let us remind the integer formulation of the (MP) that we obtained with the Dantzig-Wolfe reformulation and that we want to solve: 
(MP)

$$
\begin{array}{llr}
\max _{\lambda} & \sum_{a \in \mathcal{A}} \sum_{k \in \mathcal{K}} \sum_{j \in \mathcal{J}} \lambda_{a}^{j} \eta^{k} p_{a}^{k, j}, & \\
\text { s.t. } & \sum_{a \in \mathcal{A}} \sum_{j \in \mathcal{J}}\left[\left(c_{a}^{k}-c_{o d}^{k}\right) \lambda_{a}^{j} x_{a}^{k, j}+\lambda_{a}^{j} p_{a}^{k, j}\right]-\sum_{j \in \mathcal{J}} \lambda_{b}^{j} T_{b}^{j} \leq c_{b}^{k}-c_{o d}^{k} & \forall k \in \mathcal{K}, \forall b \in \mathcal{A}, \\
& \sum_{a \in \mathcal{A}} \sum_{j \in \mathcal{J}} \lambda_{a}^{j} x_{a}^{k, j} \leq 1 & \forall k \in \mathcal{K}, \\
& \sum_{j \in \mathcal{J}} \lambda_{a}^{j}=1 & \forall a \in \mathcal{A}, \\
& \sum_{j \in \mathcal{J}} \lambda_{a}^{j} x_{a}^{k, j} \in\{0,1\} & \forall a \in \mathcal{A}, \forall k \in \mathcal{K}, \\
& \lambda_{a}^{j} \geq 0 & \forall j \in \mathcal{J}, \forall a \in \mathcal{A} .
\end{array}
$$

As described by constraints (5.26e), the binary condition holds on original variables $x_{a}^{k}=\sum_{j \in \mathcal{J}} \lambda_{a}^{j} x_{a}^{k, j}$, for each $a \in \mathcal{A}$ and $k \in \mathcal{K}$, such that there are not "explicit" binary variables in the formulation of (MP). It is possible to add the original variables to the formulation having "explicit" binary variables to branch on, or to branch "implicitly" on constraints (5.26e). We now consider different alternatives to construct the tree, exploiting the properties of the problem.

\subsubsection{Enforcing branching decisions in the subproblem}

First of all we show how we can restrict the search space of the (SP), exploiting the branching choices. In the Branch-and-Bound tree, when solving the linear problem associated to a certain node, we would like to enforce the branching decisions of the node within the column generation procedure, such that we generate only columns that are not in contradiction with the branching decisions.

Let us define a binary branching matrix $B \in\{0,1\}^{|\mathcal{A}| \times|\mathcal{K}|}$. This matrix is associated to each node of the tree and represents the branching choices that hold in the node: $B[a][k]=0$ (resp. 1) if $x_{a}^{k}$ has been fixed to 0 (resp. 1) in the node. We also define $B[a][k]=-1$ if no branching decision has been taken yet for the pair $(a, k)$ in the node, as it will be needed later.

A column $j$, found by solving the (SP) for a certain path $a$, is in contradiction with the branching decisions if its assignment vector $x_{a}^{k, j}, k \in \mathcal{K}$, does not respect the branching decisions vector $B[a]$, i.e. if it exists a commodity $\bar{k} \in \mathcal{K}$ such that $x_{a}^{\bar{k}, j}=1$ (resp. 0 ) and $B[a][\bar{k}]=0$ (resp. 1). Such a column cannot enter the optimal solution basis of the (MP) associated to the node and therefore it does not make sense to add it to the (MP).

This is valid for both the explicit branching on original variables and the implicit branching on the constraints representing them. We present now how we can modify the solving procedure for (SP) (see Section 4.3.2 to take into account the branching decisions and generate only non contradicting columns. 
In Chapter 4, Section 4.3.2, we proposed the following rule to find the best assignment value of commodity $k$ for each toll value $T_{a} \in\left\{M_{a}^{k}: k \in \mathcal{K}\right\} \cup\{0\}$ when solving $\left(\mathrm{SP}_{a}\right)$ :

$$
x_{a}^{k}= \begin{cases}1 & \text { if } M_{a}^{k} \geq T_{a} \text { and } \pi_{a}^{k}>0 \\ 0 & \text { otherwise }\end{cases}
$$

where:

$$
\pi_{a}^{k}=T_{a}\left(\eta^{k}-\sum_{b \in \mathcal{A}} \delta_{b}^{k}\right)+\sum_{b \in \mathcal{A}} M_{a}^{k} \delta_{b}^{k}-\gamma^{k} .
$$

The following rule replaces Equation (5.27), taking into account the branching decisions:

- if $M_{a}^{k}<T_{a}$ and $B[a][k]=1$ then this column will be in contradiction with the branching decisions (we should choose $x_{a}^{k}=0$ from constraint $4.13 \mathrm{~b}$ ), so we do not consider this $T_{a}$ as a candidate solution for (SP);

- if $M_{a}^{k}<T_{a}$ and $B[a][k] \neq 1$ we choose $x_{a}^{k}=0$ by constraint $4.13 \mathrm{~b}$ of (SP);

- if $M_{a}^{k} \geq T_{a}$ and $B[a][k]=-1$ then the optimal assignment is:

$$
x_{a}^{k}= \begin{cases}1 & \text { if } \pi_{a}^{k}>0 \\ 0 & \text { otherwise }\end{cases}
$$

- if $M_{a}^{k} \geq T_{a}$ and $B[a][k] \neq-1$ then $x_{a}^{k}=B[a][k]$.

This rule finds the assignment vector that maximises the reduced price for $T_{a}$ and is not in contradiction with the branching decisions, reducing the solutions space of (SP) to columns that can be in the optimal solution of the (MP) corresponding to the node.

\subsubsection{Explicit vs implicit branching}

We now consider the problem of solving the integer (MP), in a Branch-and-Bound framework. The easiest way to solve the integer (MP) is to introduce the original variables explicitly and to branching on them. We add to (MP) the set of binary variables $x_{a}^{k}$, for all $a \in \mathcal{A}$ and $k \in \mathcal{K}$, resulting in the following formulation: 
(MP-EXP)

$$
\begin{aligned}
& \max _{\lambda} \sum_{a \in \mathcal{A}} \sum_{k \in \mathcal{K}} \sum_{j \in \mathcal{J}} \lambda_{a}^{j} \eta^{k} p_{a}^{k, j} \\
& \text { s.t. } \quad \sum_{a \in \mathcal{A}} \sum_{j \in \mathcal{J}}\left[\left(c_{a}^{k}-c_{o d}^{k}\right) \lambda_{a}^{j} x_{a}^{k, j}+\lambda_{a}^{j} p_{a}^{k, j}\right]-\sum_{j \in \mathcal{J}} \lambda_{b}^{j} T_{b}^{j} \leq c_{b}^{k}-c_{o d}^{k} \quad \forall k \in \mathcal{K}, \forall b \in \mathcal{A}, \\
& \sum_{a \in \mathcal{A}} \sum_{j \in \mathcal{J}} \lambda_{a}^{j} x_{a}^{k, j} \leq 1 \\
& \forall k \in \mathcal{K}, \quad(5.29 \mathrm{c}) \\
& \sum_{j \in \mathcal{J}} \lambda_{a}^{j}=1 \\
& \forall a \in \mathcal{A},(5.29 \mathrm{~d}) \\
& x_{a}^{k}-\sum_{j \in \mathcal{J}} \lambda_{a}^{j} x_{a}^{k, j}=0 \\
& x_{a}^{k} \in\{0,1\} \\
& \lambda_{a}^{j} \geq 0 \\
& \forall a \in \mathcal{A}, \forall k \in \mathcal{K},
\end{aligned}
$$

where constraints (5.29e) and (5.29f) replace constraints (5.26e).

The SCIP framework (Achterberg, 2009), which we are using to solve our model, integrates an automatic branching scheme when using column generation. Therefore, having implemented the column generation algorithm, (MP-EXP) can be solved to its optimal integer solution without any additional effort for the implementation.

The drawback of this strategy is that (MP-EXP) has $|\mathcal{A}| \times|\mathcal{K}|$ variables and constraints more than (MP). Numerical results presented in Section 4.3.5 showed that solving the (MP) is mainly the bottleneck of our column generation algorithm, such that increasing its size is not advised.

We tested the impact of these additional variables and constraints when solving the linear relaxation of the (HPDW) formulation, comparing the use of (MP) and (MP-EXP) for the column generation algorithm. Results are reported in Table 5.1. for the solution time in seconds and the number of generated columns.

For complete and partial graph instances we notice that including explicit original variables and the corresponding constraints increases the solution time by around $20 \%$, and the number of columns needed to find the optimal solution is more than doubled. For Shioda et al. and A1 instances the solution time remains more or less equal, whilst the number of columns almost doubles. When including original variables explicitly some Shioda et al. instances were not solved as there were numerical problems ${ }^{*}$ represents the number of instances not solved).

As the relaxed (MP-EXP) is solved in each node of the tree when using a Branchand-Price algorithm, the overall solving might be much penalised by these additional variables and constraints. Therefore, despite the simplicity of this strategy, we prefer to use model (MP) and perform the branching on constraints (5.26e). 


\begin{tabular}{|c|c|c|c|c|c|c|c|c|c|c|}
\hline \multirow{2}{*}{$\begin{array}{l}\text { Complete } \\
\text { graph }\end{array}$} & & \multicolumn{3}{|c|}{20 commodities } & \multicolumn{3}{|c|}{56 commodities } & \multicolumn{3}{|c|}{90 commodities } \\
\hline & & $20 \mathrm{a}$ & $56 a$ & $90 \mathrm{a}$ & $20 a$ & $56 \mathrm{a}$ & $90 \mathrm{a}$ & $20 a$ & $56 \mathrm{a}$ & $90 a$ \\
\hline \multirow[t]{2}{*}{ (MP) } & Time & 0.199 & 0.726 & 1.57 & 4.13 & 12.7 & 22.3 & 25.84 & 62.26 & 108.99 \\
\hline & Cols & 335 & 655 & 924 & 1141 & 1765 & 2227 & 2103 & 3034 & 3749 \\
\hline \multirow[t]{2}{*}{ (MP-EXP) } & Time & 0.235 & 0.761 & 1.659 & 4.719 & 13.80 & 25.11 & 28.09 & 72.64 & 128.51 \\
\hline & Cols & 729 & 1757 & 2715 & 2273 & 4899 & 7249 & 3889 & 8064 & 11876 \\
\hline \multirow{2}{*}{\multicolumn{2}{|c|}{$\begin{array}{l}\text { Partial } \\
\text { graph }\end{array}$}} & \multicolumn{3}{|c|}{20 commodities } & \multicolumn{3}{|c|}{56 commodities } & \multicolumn{3}{|c|}{90 commodities } \\
\hline & & $20 \mathrm{a}$ & $56 a$ & $90 \mathrm{a}$ & $20 \mathrm{a}$ & $56 \mathrm{a}$ & $90 \mathrm{a}$ & $20 \mathrm{a}$ & 56 a & $90 \mathrm{a}$ \\
\hline \multirow[t]{2}{*}{ (MP) } & Time & 0.171 & 0.687 & 1.443 & 3.026 & 10.99 & 21.23 & 18.54 & 55.71 & 102.87 \\
\hline & Cols & 301 & 625 & 891 & 974 & 1699 & 2199 & 1853 & 2926 & 3747 \\
\hline \multirow[t]{2}{*}{ (MP-EXP) } & Time & 0.192 & 0.715 & 1.572 & 3.377 & 11.895 & 24.26 & 21.03 & 64.53 & 121.18 \\
\hline & Cols & 646 & 1567 & 2409 & 1932 & 4336 & 6422 & 3409 & 7241 & 10703 \\
\hline \multirow{2}{*}{\multicolumn{2}{|c|}{$\begin{array}{l}\text { Shioda et al. } \\
\text { instances }\end{array}$}} & \multicolumn{3}{|c|}{40 commodities } & \multicolumn{3}{|c|}{60 commodities } & \multicolumn{3}{|c|}{80 commodities } \\
\hline & & $20 \mathrm{a}$ & $40 \mathrm{a}$ & $60 \mathrm{a}$ & $20 \mathrm{a}$ & $40 a^{* 1}$ & $60 \mathrm{a}$ & $20 a^{* 4}$ & $40 a^{* 1}$ & $60 a * 2$ \\
\hline \multirow[t]{2}{*}{ (MP) } & Time & 5.51 & 7.79 & 11.39 & 43.12 & 47.04 & 56.83 & 198.38 & 221.28 & 260.09 \\
\hline & Cols & 1413 & 1714 & 2015 & 2721 & 3046 & 3506 & 4586 & 5130 & 5645 \\
\hline \multirow[t]{2}{*}{ (MP-EXP) } & Time & 5.17 & 8.39 & 11.78 & 35.19 & 46.61 & 58.18 & 158.17 & 201.93 & 263.78 \\
\hline & Cols & 2186 & 3322 & 4412 & 3870 & 5494 & 7048 & 5966 & 8170 & 10416 \\
\hline \multirow{2}{*}{\multicolumn{2}{|c|}{$\begin{array}{l}\text { A1 } \\
\text { instances }\end{array}$}} & \multicolumn{3}{|c|}{21 commodities } & \multicolumn{3}{|c|}{55 commodities } & \multicolumn{3}{|c|}{91 commodities } \\
\hline & & $21 \mathrm{a}$ & $55 \mathrm{a}$ & $91 \mathrm{a}$ & $21 \mathrm{a}$ & $55 \mathrm{a}$ & $91 \mathrm{a}$ & $21 \mathrm{a}$ & $55 \mathrm{a}$ & $91 \mathrm{a}$ \\
\hline \multirow[t]{2}{*}{ (MP) } & Time & 0.07 & 0.22 & 0.46 & 0.48 & 1.69 & 3.85 & 3.14 & 8.63 & 16.45 \\
\hline & Cols & 137 & 333 & 460 & 347 & 572 & 863 & 727 & 1185 & 1527 \\
\hline \multirow[t]{2}{*}{ (MP-EXP) } & Time & 0.08 & 0.32 & 0.60 & 0.58 & 1.79 & 3.99 & 3.53 & 8.86 & 17.59 \\
\hline & Cols & 245 & 622 & 864 & 605 & 1111 & 1766 & 1208 & 2298 & 3220 \\
\hline
\end{tabular}

Table 5.1: Numerical results on the impact of explicit original $x_{a}^{k}$ variables when solving the linear relaxation of (HPDW)

\subsubsection{Implicit branching on constraints}

To avoid adding explicit original variables and corresponding constraints to (MP), we perform an implicit branching on constraints (5.26e). At each node of the branching tree, if the optimal solution of the associated linear problem is not integer, we need to decide on which pair $(a, k)$ to branch. We now present different possibilities for this choice, but first let us make a consideration on how to add branching decisions to (MP).

The most intuitive way to add a branching decision for a pair $(a, k)$, i.e. $\sum_{j \in \mathcal{J}} \lambda_{a}^{j} x_{a}^{k, j}=$ 0 (or 1), is to add this constraint to (MP). Doing so we need to take into account the associated dual variable and modify the (SP) accordingly. Moreover, adding constraints we increase the size of (MP) and we already discussed that it is not advised. There is another possibility to add a branching decision without adding a constraint: we fix some $\lambda_{a}^{j}$ variables to zero. This is possible as convexity constraints (5.26d) holds and $x_{a}^{k, j} \in\{0,1\}$ (solution of (SP)). Therefore branching decisions for a pair $(a, k)$ are added to (MP) as follows:

- for $\sum_{j \in \mathcal{J}} \lambda_{a}^{j} x_{a}^{k, j}=0$ we fix $\lambda_{a}^{j}=0$, for all $j \in \mathcal{J}$ such that $x_{a}^{k, j}=1$; 
- for $\sum_{j \in \mathcal{J}} \lambda_{a}^{j} x_{a}^{k, j}=1$ we fix $\lambda_{a}^{j}=0$, for all $j \in \mathcal{J}$ such that $x_{a}^{k, j}=0$.

Furthermore when we impose a branching decisions $\sum_{j \in \mathcal{J}} \lambda_{a}^{j} x_{a}^{k, j}=1$ for a pair $(a, k)$ we also add $\sum_{j \in \mathcal{J}} \lambda_{b}^{j} x_{b}^{k, j}=0$ for all $b \in \mathcal{A}$ such that $b \neq a$. In fact, if a commodity $k$ has been assigned to a path $a$, it cannot be assigned to any other path $b$ (constraints (5.26c)).

In the following of this section we present different strategies to choose the branching pair $(a, k)$. As there is an unambiguous correspondence between original variables $x_{a}^{k} \in\{0,1\}$ and branching constraints (5.26e), for the sake of a simpler writing we do not distinguish them.

\section{Branching on the most fractional variable}

Branching on the most fractional variable is a common simple branching rule. We choose the pair $(a, k)$ such that the corresponding original variable $x_{a}^{k}=\sum_{j \in \mathcal{J}} \lambda_{a}^{j} x_{a}^{k, j}$ is the most fractional, i.e. the one whose current fractional value is the closest to 0.5 amongst all $a \in \mathcal{A}$ and $k \in \mathcal{K}$. The current fractional value is the optimal solution of the linear (MP) associated to the current node and is denoted by $\widetilde{x}_{a}^{k}$. We choose to branch on the pair $(a, k)^{*}$ such that:

$$
(a, k)^{*}=\arg \min _{a \in \mathcal{A}, k \in \mathcal{K}}\left\{\left|\widetilde{x}_{a}^{k}-0.5\right|\right\} .
$$

Preliminary numerical tests showed that this branching scheme has very poor performance. We therefore considered more advanced branching rules, as the pseudo-cost one presented in the following section.

\section{Pseudo-costs branching rule}

The concept of pseudo-costs and estimations to generate the Branch-and-Bound tree has been introduced by Benichou et al. (1971) and pseudo-costs based branching strategies are implemented in many integer programming solvers. Pseudo-costs are empirically derived quantities, suggesting the degradation on the objective function value that will result from fixing a variable value when branching. Pseudo-costs measure in a quantitative way the importance of the different integer variables and they forecast the deterioration of the functional value when forcing an integer variable from a non integer to an integer value.

There is an assumption that pseudo-costs do not vary greatly from one part to another of the tree and if necessary they can be revised during the course of the tree search. Their justification and use are based only on experimental results, which showed that the pseudo-costs tend to remain fairly constant, having the same order of magnitude along the tree except perhaps at a few nodes.

For each pair $(a, k), a \in \mathcal{A}$ and $k \in \mathcal{K}$, we maintain two quantities, a lower pseudocost and an upper pseudo-cost, which we denote as $P_{a}^{k,-}$ and $P_{a}^{k,+}$ respectively. The 
lower (resp. upper) pseudo-cost is an estimate of the per-unit change in the objective function from forcing the value of the corresponding original variable to be rounded down to zero (resp. up to one). Let $\widetilde{x}_{a}^{k}$ be the current fractional solution value of the variable $x_{a}^{k}$ and $F$ the current objective function value. Define $F^{-}$and $F^{+}$as the objective function values obtained after solving the linear relaxation of the problem with $x_{a}^{k}=0$ and $x_{a}^{k}=1$ respectively, as represented in Figure 5.2 .

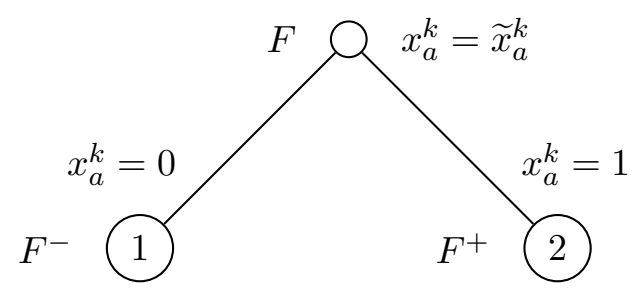

Figure 5.2: Branching on original variable $x_{a}^{k}$

Pseudo-costs of a pair $(a, k)$ are therefore defined as follows:

$$
P_{a}^{k,-}=\frac{F-F^{-}}{\widetilde{x}_{a}^{k}} \quad \text { and } \quad P_{a}^{k,+}=\frac{F-F^{+}}{1-\widetilde{x}_{a}^{k}}
$$

It may happen that in different parts of the tree we branch several times on the same pair: we keep an average of all pseudo-costs that are calculated during the solving for each pair.

Pseudo-costs have to be initialised at the root node. For each pair $(a, k)$ we set both pseudo-costs as $P_{a}^{k,-}=P_{a}^{k,+}=10 \eta^{k}$, giving more weight to pairs with higher objective function parameter.

Furthermore, during the tree search, we test different strategies to fix pseudo-costs for pairs $(a, k)$ on which no branching was performed yet:

strategy 1: keep the initial value calculated at the root node;

strategy 2: use the objective function value:

$$
P_{a}^{k,-}=\frac{\eta^{k} \widetilde{T}_{a}}{\widetilde{x}_{a}^{k}} \quad \text { and } \quad P_{a}^{k,+}=\frac{\eta^{k} \widetilde{T}_{a}}{1-\widetilde{x}_{a}^{k}}
$$

where $\widetilde{T}_{a}$ is the current fractional value of the original variable $T_{a}=\sum_{j} \lambda_{a}^{j} T_{a}^{j}$;

strategy 3: use the average of all available pseudo-costs.

From pseudo-costs we deduce an estimation of the degradation in the objective function of both children resulting from branching on a pair $(a, k)$ as follows:

$$
D_{a}^{k,-}=P_{a}^{k,-} \widetilde{x}_{a}^{k} \quad \text { and } \quad D_{a}^{k,+}=P_{a}^{k,+}\left(1-\widetilde{x}_{a}^{k}\right)
$$

Therefore, we can measure the goodness for a potential branching pair $(a, k)$ as a 
combination of the two estimated degradations. We choose the branching pair $(a, k)^{*}$ with one of the following strategies:

strategy 1:

$$
(a, k)^{*}=\arg \max _{a \in \mathcal{A}, k \in \mathcal{K}}\left\{\max \left\{0.00001, D_{a}^{k,-}\right\} \max \left\{0.00001, D_{a}^{k,+}\right\}\right\}
$$

where we pay attention of not using null values for the estimated degradation to avoid numerical problems;

strategy 2:

$$
(a, k)^{*}=\arg \max _{a \in \mathcal{A}, k \in \mathcal{K}}\left\{\alpha \min \left\{D_{a}^{k,-}, D_{a}^{k,+}\right\}+(1-\alpha) \max \left\{D_{a}^{k,-}, D_{a}^{k,+}\right\}\right\}
$$

where $0<\alpha \leq 1$ is a parameter to be set within numerical tests. If we choose $\alpha=1$ we branch on the variable maximising the minimum degradation on the subtree, meaning that we try to quickly get nodes with optimal objective value smaller than the current best integer solution such that we can cut them off and reduce the size of the tree.

Furthermore, we define "quasi-integer" variables, which have almost integer current fractional value, such that they have good chances to take integer values when forcing other variables to be integer, without the need to branch on them. These are variables for which $0<\widetilde{x}_{a}^{k} \leq \varsigma$ or $1-\varsigma \leq \widetilde{x}_{a}^{k}<1$, with $0<\varsigma<0.5$ (typically $0.1 \leq \varsigma \leq 0.2$ ). When choosing the branching pair, we first select it among corresponding non quasi-integer variables (for which $\varsigma \leq \widetilde{x}_{a}^{k} \leq 1-\varsigma$ ), and if there are none, among corresponding quasi-integer variables. This idea is in line with the most fractional variable branching rule.

Finally, pseudo-costs allow us to calculate an estimate for each waiting node, such that the node with best estimate is chosen as the following one to be solved, determining the nodes sequence in the tree exploration. When branching on a pair $(a, k)$ we denote 1 as the node obtained adding the branching decision $x_{a}^{k}=0$ and 2 as the node obtained adding the branching decision $x_{a}^{k}=1$, as represented in Figure 5.2. We propose different strategies to set the estimate of nodes 1 and 2 :

strategy 1: use the functional value of the best integer solution which can be expected at the descendant of a node:

$$
E_{1,2}=F-\sum_{a \in \mathcal{A}, k \in \mathcal{K}} \min \left\{D_{a}^{k,-}, D_{a}^{k,+}\right\}
$$

strategy 2: use the functional value of the best expected (fractional) optimal solution:

$$
E_{1}=F-D_{a}^{k,-} \quad \text { and } \quad E_{2}=F-D_{a}^{k,+}
$$


In SCIP framework (Achterberg, 2009) it is not possible to change the estimate of a node after having created it, so we need to set the estimate when choosing the branching pair $(a, k)$ and creating the two children, before solving them. Therefore we use the objective function value $F$ of the current node and not $F^{-}$and $F^{+}$.

There is also one additional parameter that we can set when creating children after branching, called priority of the node: in the tree exploration strategy, children are chosen before siblings and before leaves by priority if the estimate is good enough. This priority parameter gives preference between the two children of a node. We propose two strategies:

strategy 1: use the default strategy, i.e. both priorities set to one;

strategy 2: give more priority to the child with the smaller expected degradation, setting the priority to:

$$
P r_{1}=-D_{a}^{k,-} \quad \text { and } \quad P r_{2}=-D_{a}^{k,+}
$$

\section{SOS branching rule}

A Special Ordered Set (SOS) of type 1 is a set of variables $x_{i}, i \in \mathcal{I}$, in which at most one variable can take a positive value, all others being zero. Using binary variables it represents the problem of choosing at most on element in the set, and can be defined with the following constraints:

$$
\begin{aligned}
& \sum_{i \in \mathcal{I}} x_{i} \leq 1, \\
& x_{i} \in\{0,1\}
\end{aligned}
$$$$
\forall i \in \mathcal{I} \text {. }
$$

This concept was introduced by Beale and Tomlin (1970), where authors showed how to modify the Branch-and-Bound algorithm to take advantages of this special structure. The standard branching scheme on these variables (choose a variable and fix it to 0 in child 1 and to 1 in child 2) leads to a very unbalanced tree. In fact, fixing one variable to 1 means fixing all other variables to 0 . If there are no other integer variables in the problem, node 2 will have an integer solution and will not have any children. On the other side fixing a variable to 0 still leaves the possibility for all other variables to be positive, with a lot of possible feasible solutions for the subtree starting from node 1. The resulting tree will have the structure represented in Figure 5.3, which is highly unbalanced.

For a more balanced tree when branching on SOS variables, a more appropriate branching scheme has been introduced by Beale and Tomlin (1970). In this SOS branching rule, not yet fixed variables are divided in two disjointed sets $S$ and $S^{\prime}$. Then in the child node 1 we impose $\sum_{i \in S} x_{i}=0$ (which means $x_{i}=0, \forall i \in S$ ) and in the child node 2 we impose $\sum_{i \in S^{\prime}} x_{i}=0$ (which means $x_{i}=0, \forall i \in S^{\prime}$ ), as represented in Figure 
5.4

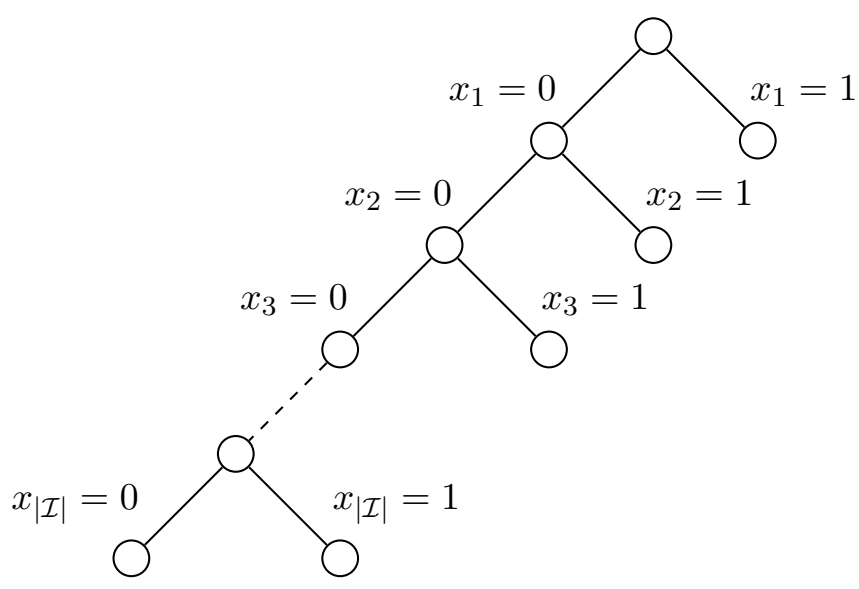

Figure 5.3: Tree for standard branching on SOS variables

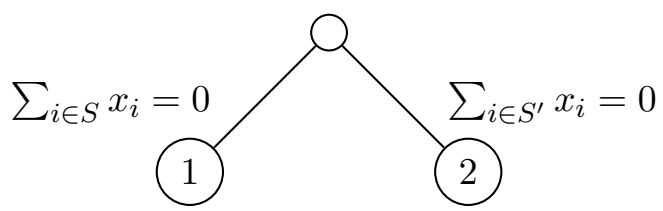

Figure 5.4: SOS branching rule

If sets $S$ and $S^{\prime}$ are appropriately defined, the resulting tree is balanced, leading to a better performing branching algorithm. We now detail how a SOS branching rule applies to our problem.

In the (MP) we have the following two sets of constraints:

$$
\begin{array}{ll}
\sum_{a \in \mathcal{A}} \sum_{j \in \mathcal{J}} \lambda_{a}^{j} x_{a}^{k, j} \leq 1 & \forall k \in \mathcal{K}, \\
\sum_{j \in \mathcal{J}} \lambda_{a}^{j} x_{a}^{k, j} \in\{0,1\} & \forall a \in \mathcal{A}, \forall k \in \mathcal{K},
\end{array}
$$

such that for each commodity $k \in \mathcal{K}$ original variables $x_{a}^{k}=\sum_{j \in \mathcal{J}} \lambda_{a}^{j} x_{a}^{k, j}, a \in \mathcal{A}$, form a SOS of type 1 .

We therefore define the following SOS branching rule to construct our tree. At each node:

- choose the commodity $k$ with the maximum number of fractional values for original variables $x_{a}^{k}$;

- for each $a \in \mathcal{A}$ such that no branching decision has been taken yet for the pair $(a, k)$ : 
- if the fractional value $\widetilde{x}_{a}^{k}>0$ :

$$
\begin{array}{ll}
a \in S & \text { if } \quad \widetilde{x}_{a}^{k}+\sum_{b \in S} \widetilde{x}_{b}^{k} \leq 0.5 \\
a \in S^{\prime} & \text { otherwise }
\end{array}
$$

- if the fractional value $\widetilde{x}_{a}^{k}=0$ :

$$
a \in S \quad \text { if } \quad S^{\prime}=\emptyset
$$

otherwise we randomly choose between $S$ and $S^{\prime}$

Then, in the child node 1 we impose:

$$
\sum_{a \in S} \sum_{j \in \mathcal{J}} \lambda_{a}^{j} x_{a}^{k, j}=0 \quad \Rightarrow \quad \sum_{j \in \mathcal{J}} \lambda_{a}^{j} x_{a}^{k, j}=0 \quad \forall a \in S
$$

and in the child node 2 we impose:

$$
\sum_{a \in S^{\prime}} \sum_{j \in \mathcal{J}} \lambda_{a}^{j} x_{a}^{k, j}=0 \quad \Rightarrow \quad \sum_{j \in \mathcal{J}} \lambda_{a}^{j} x_{a}^{k, j}=0 \quad \forall a \in S^{\prime}
$$

\subsection{Additional stopping criterion using dual bound for the in- teger problem}

In Section 4.3 .3 we introduced a dual upper bound for the optimal value of the linear relaxation of (MP), that can be calculated at each iteration of the column generation algorithm. Furthermore, in Section 4.8 we showed how to use it as an additional stopping criterion for the column generation algorithm. Numerical results however showed that using it does not have a great impact on the performance of the algorithm. Nevertheless, as it does not degrade it, we included this criterion in our algorithm.

In this section we propose another stopping criterion that uses this dual bound, while solving one node of the branching tree. Let us denote $U B$ as the dual upper bound of the relaxed (MP) that we are solving in the node, which can be calculated as follows (for more details we refer to Chapter 4, Section 4.3.3, where we introduced this bound):

$$
U B=\sum_{k \in \mathcal{K}} \gamma^{k}+\sum_{a \in \mathcal{A}} \mu_{a}-\sum_{b \in \mathcal{A}} \sum_{k \in \mathcal{K}} M_{b}^{k} \delta_{b}^{k}+\sum_{a \in \mathcal{A}} \mathrm{RP}_{a},
$$

and $Z_{I N T}$ as the best integer solution that we found so far during the tree exploration.

If at a certain iteration of the column generation algorithm (while solving the relaxed restricted (MP) of the node) we have that $U B<Z_{I N T}$, we can stop the column generation algorithm to solve the node and cut off this node. In fact, as $U B$ is an upper bound on the optimal solution of the relaxed (MP) of the node, we are guaranteed that the optimal solution will not be in this node or in its children, as any solution in this sub-tree will be smaller than the best integer solution found so far. Note that the 
node would be in any case cut off by the solver from the standard branch-and-bound rules, but with this criterion we avoid solving it until the end of the column generation algorithm.

\subsection{Integrating heuristic solutions in the Branch-and-Price}

In this section we discuss how to integrate integer solutions found with heuristic algorithms in the Branch-and-Price framework. Consider the heuristic algorithms we proposed in Section 5.4, and assume that we have a feasible solution from either Heuristic 1 or 2 . Let us denote $Z_{H}$ as its objective function value, $T_{a}$ as the toll values of paths and $x_{a}^{k}$ the assignment values of commodities to paths.

First of all we first check if $Z_{H}>Z_{I N T}$, i.e. if the solution found by heuristic is better than the best known integer solution. If it is the case we use this solution in the Branch-and-Price algorithm. The easiest way is to simply use $Z_{H}$ as the new lower bound for the optimal integer solution: we pass this information to the solver that will add it to its rules for cutting off nodes, and we store the toll and assignment values of this solution in case it is the optimal one.

Another possibility is to include the complete solution in the algorithm, i.e. create a set of feasible columns that correspond to it and pass to the solver the information that these columns correspond to a feasible integer solution. Then the solver will automatically update the bound values. This option increases the number of columns and also affects the solution process of the linear problems, as we add variables. However without testing the behaviour of the algorithm on the instances we do not know if this might improve or deteriorate the performance.

Before constructing the set of feasible columns, we first need to check that the heuristic solution is not in contradiction with the branching decisions of the current node: the assignment matrix found by the heuristic does not respect the branching decisions matrix if it exists a path $\bar{a} \in \mathcal{A}$ and a commodity $\bar{k} \in \mathcal{K}$ such that $x_{\bar{a}}^{\bar{k}}=1$ (resp. 0 ) and $B[\bar{a}][\bar{k}]=0$ (resp. 1), as we introduced in Section 5.5.1. If the heuristic solution is in contradiction with the branching decisions we cannot create a corresponding set of feasible columns in the node and we update only the integer lower bound as described above. Otherwise, we use the following procedure:

- create one new column $j$ for each toll path $a \in \mathcal{A}$, with the following parameters:

- $T_{a}^{j}=T_{a} ;$

- $x_{a}^{k, j}=x_{a}^{k}$, for each $k \in \mathcal{K}$;

- $p_{a}^{k, j}=T_{a} x_{a}^{k}$, for each $k \in \mathcal{K}$;

- set the variables corresponding to these new columns $\lambda_{a}^{j}=1$, and all other variables equal to zero;

- pass to the solver the information that this is a integer feasible solution. 


\subsection{Branch-and-Cut-and-Price framework}

In Chapter 4 we introduced various tools to improve the column generation algorithm to solve the linear relaxation of (HPDW), such as different stabilisation strategies, initialisation alternatives, etc. Valid inequalities were also reformulated and added to improve the gap at the root node. In this chapter, Section 5.4. we proposed heuristic algorithms to find integer solutions during the tree exploration. Finally we analysed how to use these elements in a Branch-and-Bound framework, and we proposed various branching rules to construct the tree. All these elements have been merged leading to a Branch-and-Cut-and-Price (B\&C\&P) algorithm for the NPP with connected toll arcs or (HP). We name this algorithm as HP-B\&C\&P and we now describe its features.

\subsubsection{List of parameters to define the HP-B\&C\&P algorithm}

For a sake of completeness we report again the parameters introduced in Section 4.10 .1 for the column generation algorithm to solve the linear relaxation of (HPDW):

-c strategy to initialise columns for the column generation algorithm (see Section 4.6.1). Possible values $\mathrm{c}=\{1,2\}$.

1: strategy INIT 0 (all null columns), 2: strategy INIT 1 (with heuristic).

-v maximum number of columns added at each iteration of column generation, for each path (see Section 4.7). Possible values $v=\{1,2, \ldots,|\mathcal{K}|\}$.

-s stabilisation strategy (see Section 4.9.2). Possible values $s=\{0,1,2,3,4,5,6,7,8,9,10\}$. 0: stabilisation disabled, 1: Strategy 1 (1-gap), 2: Strategy 2 (from Pessoa et al., 2010)), 3: Strategy 3 (mixed 1 and 2), 4: Strategy 4 (fixed), 5: Strategy 5 (autoadaptive with subgradient, from Pessoa et al. (2013)).

Strategies $6,7,8,9,10$ are as $1,2,3,4,5$ but they are active only at root note.

Only if $s>0$ :

-e parameter $\bar{\varepsilon}$ to disable stabilisation when the gap is smaller than this epsilon.

Possible values $0.00001<e<1$.

Only if $\mathrm{s}=\{1,3,4,6,8,9\}$ :

-d parameter $\Delta$ for the convex combination of dual values during stabilisation. Possible values $0.00001<d \leq 1$.

-m columns are removable due to ageing. Possible values $\mathbf{m}=\{0,1\}$.

0 : not removable, 1: removable.

Only for $\mathrm{m}=1$ :

-a age after which columns are removed. Possible values $\mathbf{a}=\{0,1,2,3, \ldots\}$. 
-S algorithm for solving initial LP relaxations. Possible values $\mathbf{S}=\{0,1,2\}$.

0: automatic simplex, 1: primal simplex, 2: dual simplex.

- $\mathbf{R}$ algorithm for resolving LP relaxations if a starting basis exists. Possible values $\mathrm{R}=\{0,1,2\}$.

0: automatic simplex, 1: primal simplex, 2: dual simplex.

Note that the stabilisation parameter "s" has more values for the HP-B\&C\&P algorithm than for the column generation algorithm, as Strategies 2 and 4 and Strategies 1 and 3 differ from each other when solving the integer problem, and we also consider new strategies using the stabilisation only at the root node and not in the tree nodes.

We then add the following parameters to define the branching rule used to construct the tree:

-b implicit branching rule (see Section 5.5.3). Possible values $b=\{1,2,3\}$.

1: branching on most fractional variable, 2: pseudo-costs branching rule, 3: SOS branching rule.

Only for $b=2$ (when using pseudo-costs):

-n strategy to fix pseudo-costs for variables on which no branching was performed yet. Possible values $\mathrm{n}=\{1,2,3\}$.

1: Strategy 1 (keep initial value), 2: Strategy 2 (use functional value), 3: Strategy 3 (average of all available values).

-p rule to choose the branching pair, with the estimated degradations. Possible values $\mathrm{p}=\{1,2\}$.

1: Strategy 1 (product), 2: Strategy 2 (weighted sum).

Only for $\mathrm{p}=2$ :

-q parameter $\alpha$ for the weighted sum. Possible values $0<q \leq 1$.

-f parameter $\varsigma$ to define quasi integer variables. Possible values $0.00001 \leq f<$ 0.5 .

-g rule to calculate the estimate value for the new nodes. Possible values $g=\{1,2\}$. 1: Strategy 1 (best expected integer solution), 2: Strategy 2 (best expected fractional solution)

-r rule to calculate priority between the two new children. Possible values $r=\{1,2\}$. 1: Strategy 1 (default), 2: Strategy 2 (expected degradation).

As described in Section 5.7 we can include our heuristic algorithms in the HP$\mathrm{B} \& \mathrm{C} \& \mathrm{P}$ framework, adding the following parameters:

-h activation of the Heuristic 1 (see Sections 5.4.1 and 5.7). Possible values $h=\{0,1,2\}$.

0 : not active, 1: active adding columns corresponding to solution found (if good), 
2: active but only improve the primal lower (integer) bound (if good solution found).

-k activation of the Heuristic 2 (see Sections 5.4.2 and 5.7). Possible values $\mathbf{k}=\{0,1,2\}$.

0 : not active, 1: active adding columns corresponding to solution found (if good),

2: active but only improve primal lower (integer) bound (if good solution found).

Only for k > 0:

-j frequency parameter for calling Heuristic 2 . Possible values $j=\{1,2,3, \ldots\}$.

1: called at each node, 2 : called only at nodes of depth $1,3,5,7, \ldots, 3$ : called only at nodes of depth $1,4,7,10, \ldots, 4$ : called only at nodes of depth $1,5,9,13, \ldots$, etc.

And finally we include the possibility of adding the valid inequalities to improve the gap at the root node. Similarly as for variables (see the description before parameters " $\mathrm{m}$ " and "a" in Section 4.10.1), in SCIP it is possible to "remove" rows (i.e. cuts) due to ageing. We add the following parameters:

$\mathbf{- z}$ activation of (SSPI) valid inequalities (see Section 4.11). Possible values $\mathbf{z}=\{0,1\}$.

0 : not active, 1: active with the first separation strategy, 2: active with the second separation strategy.

Only for $z>0$ :

-w cuts are removable due to ageing. Possible values $\{0,1\}$.

0 : not removable, 1: removable.

Only for $\mathrm{w}=1$ :

-y age after which cuts are removed. Possible values $\{0,1,2,3, \ldots\}$.

\subsubsection{Automatic tuning of the parameters}

Our HP-B\&C\&P framework has several (23) parameters to be chosen such that manually testing all the possible configurations would require a big effort. Therefore, to determine its best configuration, as we already did to tune the column generation algorithm in Chapter 4. Section 4.10.2, we use again irace, which is a tool for the automatic configuration of algorithms developed by López-Ibánez et al. (2011) from IRIDIA research group (ULB). Also the tuning of the HP-B\&C\&P algorithm was performed by Leslie Pérez Cáceres from IRIDIA.

As solving the integer (HPDW) requires a remarkable computational time, we decide to perform only one tuning run for each set of instances and we are able to use only the smaller instances, using a time budget of 1500 seconds to solve each instance with a specific configuration. Moreover, we restrict the research space for some parameters, 
either as we know that some configurations are very bad or because for some integer parameters there is no big difference between subsequent values. In particular we use the following restrictions:

-b we use only $\{2,3\}$, as we know from preliminary tests that the branching on the most fractional variable is performing very badly;

-v we use values $\{1,2,3,4,5,10,20\}$ for complete and partial graph instances, $\{1,2,3,4,5,10,20,40\}$ for Shioda et al. instances and $\{1,2,3,4,5,10,21,40,55\}$ for A1 instances, as the algorithm is mainly affected by adding few or many columns;

-m we use values $\{0,1,2,3,4,5,7,10,15,20,25,30,40,50\}$, as we want to test if it is effective to remove columns immediately as they go out of basis or after many iterations.

Moreover, we first test the pure Branch-and-Price algorithm, without adding cuts, and so setting parameter $\mathrm{z}=0$.

We tune the algorithm on the complete and partial graph sets using all instances with 20 commodities, on the Shioda et al. set with 40 commodities and on the A1 set with 21 and 55 commodities as these instances are quicker to solve. The tuning on complete graph instances requires around 3 days, whilst for the partial graph instances it requires around 5 days and many configurations run out of memory, such that tuning gives as best configurations the ones that do not use too much memory. For Shioda et al. instances the tuning does not converge and does not find meaningful results, as all configurations run out of memory. Finally for A1 instances the tuning runs smoothly and requires around 2 days.

In Table 5.2 we report the six best configurations found by the tuner (in order with the best one first), for complete and partial graph and A1 instances.

Note that we do not report the tuning results for Shioda et al. instances as we do not have any meaningful result, and that the reported results for partial graph instances have to be used carefully as the tuning does not run correctly also for this class of instances, as explained above. Therefore we decide to first analyse and test the configurations found by the tuning for the complete graph and A1 instances, and if results are promising, to continue with the two missing classes.

Consider first the best configurations found for the complete graph instances: from Table 5.2 we notice that they mainly differ on the initialisation (parameter "-c") and stabilisation strategies (parameter "-s"), as some configuration use the first initialisation strategy and some the second, without any predominance, and some configurations use the stabilisation strategy 8 (a mix of the "1-gap" one and the one from Pessoa et al. (2010)) and some the 10 (the auto-adaptive with subgradient one), both active only at the root node. In any case the stabilisation is not used much, as the $\bar{\varepsilon}$ parameter ("-e" in the table, which disables it) is quite high. The other parameters choices are fairly 
Complete graph instances $(20 \mathrm{k})$

\begin{tabular}{|c|c|c|c|c|c|c|c|c|c|c|c|c|c|c|c|c|c|c|c|}
\hline Conf & $-\mathrm{c}$ & $-\mathrm{v}$ & $-s$ & $-d$ & -e & $-\mathrm{m}$ & $-a$ & $-S$ & $-\mathrm{R}$ & $-b$ & $-n$ & $-p$ & $-q$ & $-f$ & $-g$ & $-r$ & $-\mathrm{h}$ & $-\mathrm{k}$ & $-\mathrm{j}$ \\
\hline 1 & 1 & 1 & 8 & 0.99 & 0.78 & 0 & - & 0 & 2 & 2 & 3 & 1 & - & 0.14 & 1 & 1 & 2 & 0 & - \\
\hline 2 & 2 & 1 & 10 & - & 0.62 & 0 & - & 2 & 2 & 2 & 3 & 1 & - & 0.22 & 1 & 1 & 2 & 0 & - \\
\hline 3 & 1 & 1 & 10 & - & 0.61 & 0 & - & 2 & 2 & 2 & 3 & 1 & - & 0.15 & 1 & 1 & 2 & 0 & - \\
\hline 4 & 2 & 1 & 10 & - & 0.68 & 0 & - & 2 & 2 & 2 & 3 & 1 & - & 0.14 & 1 & 1 & 2 & 0 & - \\
\hline 5 & 1 & 1 & 8 & 0.41 & 0.56 & 0 & - & 2 & 2 & 2 & 3 & 1 & - & 0.21 & 1 & 1 & 2 & 0 & - \\
\hline 6 & 2 & 1 & 10 & - & 0.79 & 0 & - & 0 & 2 & 2 & 3 & 1 & - & 0.02 & 1 & 1 & 2 & 0 & - \\
\hline \multicolumn{20}{|c|}{ Partial graph instances (20k) } \\
\hline Conf & $-\mathrm{c}$ & $-\mathrm{v}$ & $-s$ & $-\mathrm{d}$ & -e & $-m$ & $-\mathrm{a}$ & $-S$ & $-\mathrm{R}$ & $-b$ & $-n$ & $-p$ & $-q$ & $-f$ & $-g$ & $-r$ & $-h$ & $-k$ & $-j$ \\
\hline 1 & 1 & 1 & 3 & 0.05 & 0.88 & 0 & - & 0 & 2 & 2 & 1 & 1 & - & 0.14 & 1 & 1 & 0 & 0 & - \\
\hline 2 & 1 & 1 & 3 & 0.07 & 0.68 & 0 & - & 0 & 2 & 2 & 1 & 1 & - & 0.30 & 1 & 1 & 0 & 0 & - \\
\hline 3 & 2 & 1 & 3 & 0.06 & 0.38 & 0 & - & 0 & 2 & 2 & 1 & 1 & - & 0.34 & 1 & 1 & 2 & 0 & - \\
\hline 4 & 2 & 1 & 3 & 0.05 & 0.40 & 0 & - & 0 & 2 & 2 & 1 & 1 & - & 0.34 & 1 & 1 & 2 & 0 & - \\
\hline 5 & 1 & 1 & 3 & 0.06 & 0.58 & 0 & - & 0 & 2 & 2 & 1 & 1 & - & 0.44 & 1 & 1 & 0 & 0 & - \\
\hline 6 & 2 & 1 & 3 & 0.29 & 0.14 & 0 & - & 0 & 2 & 2 & 1 & 1 & - & 0.22 & 1 & 1 & 2 & 0 & - \\
\hline \multicolumn{20}{|c|}{ A1 instances (21k and 55k) } \\
\hline Conf & $-\mathrm{c}$ & $-\mathrm{v}$ & $-s$ & $-\mathrm{d}$ & $-\mathrm{e}$ & $-m$ & $-\mathrm{a}$ & $-S$ & $-\mathrm{R}$ & $-b$ & $-n$ & $-p$ & $-q$ & $-f$ & $-g$ & $-r$ & $-h$ & $-\mathrm{k}$ & $-j$ \\
\hline 1 & 2 & 1 & 6 & 0.85 & 0.78 & 0 & - & 2 & 2 & 2 & 1 & 2 & 0.88 & 0.06 & 2 & 2 & 2 & 2 & 7 \\
\hline 2 & 2 & 1 & 6 & 0.54 & 0.68 & 0 & - & 2 & 2 & 2 & 1 & 2 & 0.95 & 0.04 & 2 & 2 & 2 & 2 & 6 \\
\hline 3 & 2 & 1 & 6 & 0.75 & 0.86 & 0 & - & 2 & 2 & 2 & 1 & 2 & 0.96 & 0.02 & 2 & 2 & 2 & 2 & 7 \\
\hline 4 & 2 & 1 & 3 & 0.56 & 0.86 & 0 & - & 2 & 2 & 2 & 1 & 2 & 0.96 & 0.07 & 2 & 1 & 2 & 2 & 8 \\
\hline 5 & 2 & 1 & 6 & 0.77 & 0.93 & 0 & - & 2 & 2 & 2 & 1 & 1 & - & 0.14 & 2 & 2 & 2 & 2 & 7 \\
\hline 6 & 2 & 1 & 6 & 0.56 & 0.86 & 0 & - & 2 & 2 & 2 & 1 & 2 & 0.86 & 0.22 & 2 & 2 & 2 & 0 & - \\
\hline
\end{tabular}

Table 5.2: Best configurations of parameters for each instances set irace results

constant: we always add one column for each path at each iteration of the column generation algorithm ("- $\mathrm{v}=1$ "), never remove out of basis columns ("- $\mathrm{m}=0$ "), always use the dual simplex to solve LPs ("-S=0/2" and "-R=2"), always use pseudo-cost values to construct the tree ("-b=2"), using the average of available pseudo-costs to update the not yet available ones ("- $n=3$ "), the product of the degradations to choose the branching pair (" $-\mathrm{p}=1$ "), a value of around $0.15-0.20$ for quasi-integer variables ("- $\mathrm{f}$ "), the best expected integer solution as the estimate for new nodes (" $-\mathrm{g}=1$ ") and the default priority for the two children ("-r=1"). Finally we activate the first heuristic ("-h=2"), only updating the primal bound when we find good solutions, and not adding the columns corresponding to the solutions found, but we do not activate the second heuristic ("$\left.\mathrm{k}=0^{\prime \prime}\right)$.

Consider now the best configurations found for A1 istances (Table 5.2): we notice that they are all very similar. All configurations use the second initialisation strategy $("-c=2$ "), and the stabilisation strategy $6("-s=6$ "), which is the "1-gap" one active only at the root node, with a high value of $\Delta$ and $\bar{\varepsilon}$ ("- $\mathrm{d}$ " and "-e" in the table, respectively), 
which means that the stabilisation is not used much. As for the other class of instances, we have "-v=1", "-m=0", "-S=2" and "-R=2". We also use pseudo-cost values for the branching ("- $b=2$ "), but we keep the initial values for not yet available pseudo-costs ("-n=1") and we use a weighted sum of the degradations to choose the branching pair, giving more weight to the minimum degradation to try to quickly cut off the subtree ("- $-\mathrm{p}=2$ " and high " $-\mathrm{q}$ "). Quasi-integer variables are defined with a value of around $0.05\left(\right.$ " $\left.-f^{\prime \prime}\right)$, we use the best expected optimal solution as the estimate for new nodes $("-g=2 ")$ and we give more priority to the child with the smaller expected degradation $("-r=2$ "). Finally, in addition to the first heuristic ("-h=2"), we activate also the second one, always only updating the primal bound and not adding any new column ("'-k=2"), calling it only in nodes of depth 1,8,15,22,... (" $-\mathrm{j}=7$ ").

Given these considerations, for complete graph instances we test the performance of the first and the second configurations of parameters found by the automatic tuning, whilst for A1 instances we test only the first configuration. To assess the goodness of the tuning results, in the next section we compare them with a configuration that we chose thinking that it should be reasonably good.

\subsubsection{Numerical results}

In this section we report numerical results when solving the integer (HP). We compare using the (HPL) formulation and (HPDW) formulation, solved with different configurations of the HP-B\&C\&P algorithm. In particular for the HP-B\&C\&P we use the following configurations:

"Try" : a configuration that we think could be good, based on results on the column generation algorithm and our intuition, which is as follows:

$$
\begin{array}{llllllll}
-\mathrm{c}=2 & -\mathrm{v}=1 & -\mathrm{s}=5 & -\mathrm{e}=0.05 & -\mathrm{m}=0 & -\mathrm{S}=0 & -\mathrm{R}=2 & \\
-\mathrm{b}=2 & -\mathrm{n}=1 & -\mathrm{p}=1 & -\mathrm{f}=0.2 & -\mathrm{g}=1 & -\mathrm{r}=2 & -\mathrm{h}=1 & -\mathrm{k}=0
\end{array}
$$

"Tun1", “Tun2": configurations found by the tuning, where "Tun1" means the first configuration and "Tun2" the second for each type of instance (see Table 5.2 for the parameter values of each of them).

Furthermore, for both (HPL) and (HPDW) we test the impact of adding the set of valid inequalities SSPI described in Section 1.5.8 and reformulated for (HPDW) in Section 4.11. Note that, for both (HPL) and (HPDW), when adding the SSPI (noted as +SSPI in the tables) we use the second separation strategy, as we saw that it provides a root gap improvement similar to the first separation strategy, with the advantage of adding less inequalities (see Section 4.11 for more details). 
In Tables 5.3 and 5.4 we report numerical results for these models and configurations, for complete graph and A1 instances, respectively. We report the average solution time in seconds for each instance size (noted as "Time"), only for instances that are solved within the time limit of 5 hours (or 18000 seconds) that we impose: ${ }^{* n}$ represents the number of instances that are not solved within this limit, and if we do not report any time value it means that none of the instances were solved in the limit. Then we report the average number of nodes of the branching tree (notes as "Nodes") and the time at which the optimal solution was found (noted as "Time OptSol"). For (HPDW) we also report the number of generated columns (as "Cols"), the number of total iterations of the column generation algorithm (as "Iter"), the time spent by the algorithm to solve the subproblem (noted as "(SP) Time"), and the number of instances in which the heuristics, if active only updating the primal bound, found the optimal solution (noted as "SolByHeur Instances"). Finally, for instances for which no model was able to find and prove the optimal solution within the time limit, we report the gap still left to be closed after 5 hours, noted as "NotSolved FinalGap". Note that the time spent by the algorithm to solve the (MP) can be obtained subtracting the (SP) time from the total time, as the other features of the algorithm (branching, heuristics, ...) use a non significant amount of time.

For an easier analysis of the results, we also report again at the top of each table the root node gap of both formulations, with and without SSPI. For the complete description of root node gap results we refer to Sections 4.5.2 and 4.11.4. but let us remind that this value is the gap in percentage between the optimal integer solution value and the root node linear relaxation optimal value.

Furthermore, to compare (HPL) and (HPDW) formulations for A1 instances (both with or without SSPI), we report the number of instances for which (HPDW), with the tuned configurations of the HP-B\&C\&P algorithm, was solved faster than (HPL), and the number of instances for which the optimal solution was found by (HPDW) before than (HPL), noted as "DW < L:", "Time Instances" and "TimeOptSol Instances", respectively.

Then, for an easier comparison between the different configurations of the Branchand-Price algorithms, we compare the best integer solution value that two configurations were able to find, and report the number of instances for which the first provided a higher solution than the second (noted as "BestSolVal Tun1 > Try", "BestSolVal Tun2 $>$ Try1", etc. in the table).

In Figures 5.5 and 5.6 we report the performance profile graphs for the formulations and configurations of Tables 5.3 and 5.4 respectively, where the y-axis represents the number of instances solved within the corresponding time (in seconds in logarithmic scale) in the x-axis, such that the higher the curve the better the formulation/configuration is performing.

First we compare the different configurations of the HP-B\&C\&P algorithm to solve (HPDW) formulation: as expected, for both classes of instances we clearly see that the 


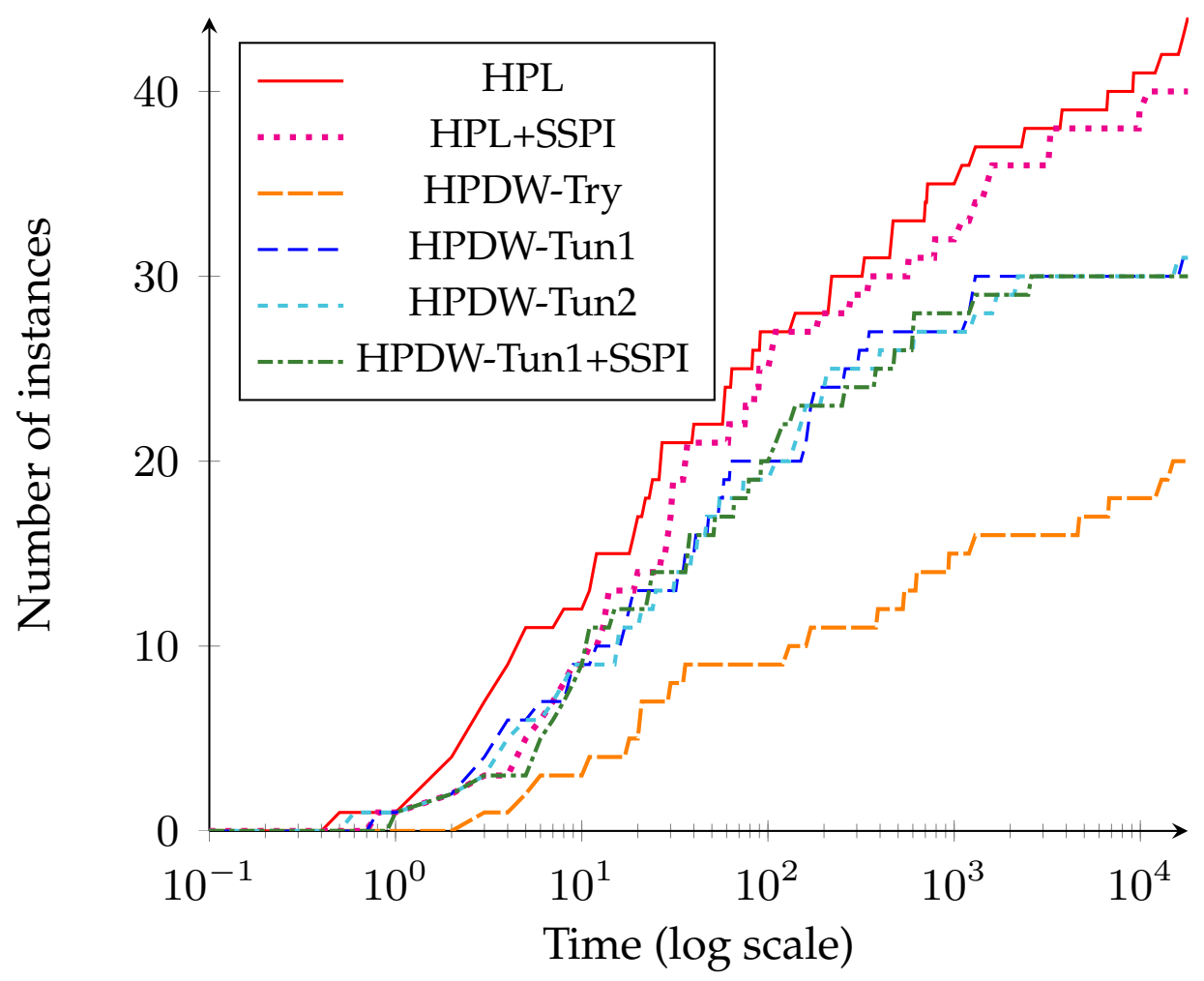

Figure 5.5: Performance profile graphs on solving (HP) for complete graph instances, comparing (HPL) and (HPDW) with different configurations of HP-B\&C\&P

best configurations found by the tuning with irace outperform our guessed good configuration "Try".

For complete graph instances we tested two different configurations found by the tuner, as they are a bit different, but we can see that their performances are very similar, with the first one (which was the best for the tuner) being slightly better. With these two configurations ("Tun 1" and "Tun 2") we are able to solve to optimality all 20 commodities instances, whilst with the "Try" configuration we only solved two thirds of them. The solution time is reduced by a factor of ten, even if the number of nodes of the branching tree increases. This increase in the number of nodes is however also due to the fact that we solve more instances, so also the more difficult ones for each size, which require more nodes. The number of generated columns is significantly reduced, whilst the number of iterations increases: this allows us to have smaller (MP)s to solve at each iteration, and in fact we can calculate from the results in the Table 5.3 that for instances with 20 commodities and 90 toll paths each (MP) takes in average 0.75 seconds to be solved for "Try", and 0.02 seconds for "Tun 1" and "Tun 2" (each (SP) takes around 0.02 seconds for all configurations). For instances that are not solved to optimality, "Tun 1" and "Tun 2" configurations also reduce the gap still left after the time limit, even though it is still quite high. Moreover, "Tun 1" and "Tun 2" very often find better integer solutions than "Try" configuration. Out of these 60 instances that were not solved by both "Tun 1" and "Tun 2" (the ones with 56 and 90 commodities), 


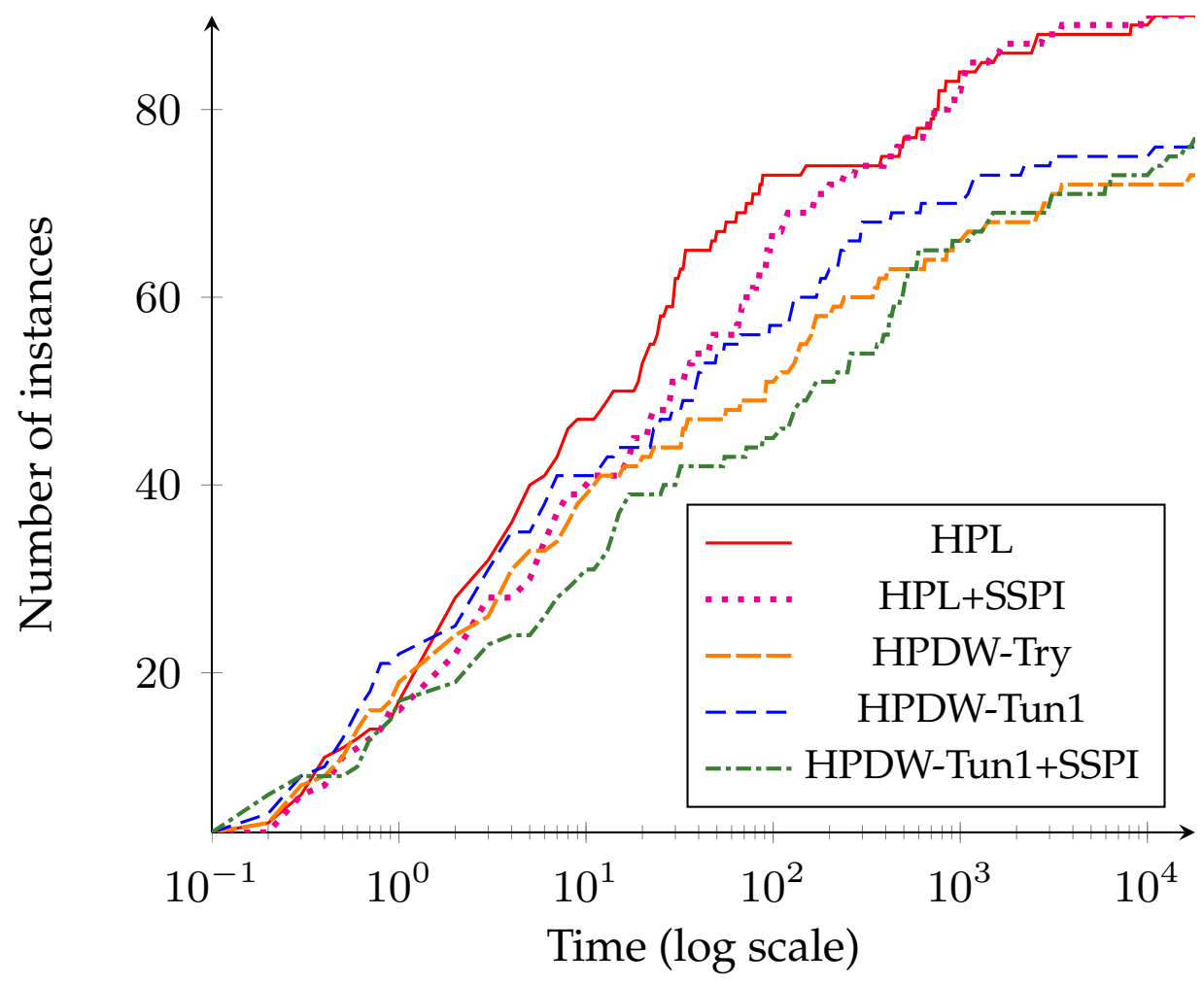

Figure 5.6: Performance profile graphs on solving (HP) for A1 instances, comparing (HPL) and (HPDW) with different configurations of HP-B\&C\&P

"Tun 1" finds a better integer solution than "Tun 2" in 33 cases, and almost always the heuristic finds the best solution for both configurations in a very small time (less than one second). The heuristic also finds many of the optimal solutions for the smaller instances.

For A1 instances we tested only the best configuration found by the tuner, as they are all quite similar, noted as "Tun 1 ". With this configuration we solve all instances except 14 of the 91 commodities, 3 more with respect to the "Try" configuration. Also for this class of instances the solution time is significantly decreased by "Tun 1", in particular by a factor of ten for instances with 91 commodities and 20 toll paths. Let us remark that the increase in the solution time for instances with 91 commodities and 55 or 91 toll paths is due to the fact that we solve more instances (and so more difficult ones), and we report the average. The considerations for the number of nodes, columns and iterations, and for the time to solve each (MP) are similar as for the other class of instances, so we do not repeat them. We just underline that for the bigger A1 instances each (MP) is solved in average in 0.04 seconds, whilst for the bigger complete graph instances it takes around 0.24 seconds: we already noticed this difference when testing the column generation algorithm to solve the linear relaxation of the problem, in Section 4.10.3. Also for A1 instances the heuristic finds most of the optimal solutions.

Compare now the (HPDW) and (HPL) formulations: from the graphs of Figures 5.5 and 5.6 we notice that (HPL) solves more instances and in less time, for both classes of 
instances. However, for some small A1 instances (HPDW) with "Tun 1" is solved more quickly than (HPL): this is true for 20 out of the 30 instances with 21 commodities and for 5 out of the 30 instances with 55 instances. Moreover, for complete graph instances, (HPDW) with "Tun 1" or "Tun 2" is the only formulation able to solve to optimality within the time limit one instance with 56 commodities and toll paths (note that it is not the same instance for the two configurations). Additionally, for A1 instances, the time at which the optimal solution is found is very often smaller for (HPDW) than for (HPL): this is true for 54 out of the 90 instances and often (HPDW) finds the optimal solution in fractions of a second, whilst (HPL) requires up to hundreds of seconds. One could think to run these two formulations in parallel and pass the best integer solutions found by (HPDW) to (HPL), allowing the Branch-and-Bound algorithm used to solve (HPL) to cut off some nodes, reducing the tree size and therefore probably improving its solution time.

If we add SSPI to both formulations we notice that (HPL)'s performance is improved for the larger instances, whilst (HPDW) performance is quite deteriorated. We already saw that the root gap improvement of adding SSPI is much more significant for (HPL) than for (HPDW). Numerical results show that adding these inequalities does not integrate well with the column generation and Branch-and-Price algorithms.

To conclude, despite the promising better root node gap and the significant improvements that we provide for the column generation and Branch-(and-Cut)-andPrice algorithms, the overall performance of this framework in solving the problem is not better than the mixed-integer formulation that was proposed in the literature. However, on A1 instances, which are more realistic for the NPP as they have an underlying network, there are some positive results, in particular on the small time needed by the Branch-and-Price algorithm to find the optimal solution. We also noticed that for these instances the (MP) is relatively quick to solve, so the bottleneck seems to be the (SP): if some improvements are found on its solution procedure, then the Branch-and-Price algorithm to solve (HPDW) might reveal better performance. 


\begin{tabular}{|c|c|c|c|c|c|c|c|c|c|c|}
\hline \multirow{2}{*}{$\begin{array}{l}\text { Complete } \\
\text { graph }\end{array}$} & & \multicolumn{3}{|c|}{20 commodities } & \multicolumn{3}{|c|}{56 commodities } & \multicolumn{3}{|c|}{90 commodities } \\
\hline & & $20 \mathrm{a}$ & $56 \mathrm{a}$ & $90 \mathrm{a}$ & $20 \mathrm{a}$ & $56 a$ & $90 \mathrm{a}$ & $20 \mathrm{a}$ & $56 \mathrm{a}$ & $90 \mathrm{a}$ \\
\hline (HPL) & RootGap & $6.19 \%$ & $3.71 \%$ & $3.15 \%$ & $12.82 \%$ & $11.18 \%$ & $11.51 \%$ & $15.33 \%$ & $16.88 \%$ & $17.47 \%$ \\
\hline +SSPI & RootGap & $2.36 \%$ & $1.91 \%$ & $1.51 \%$ & $4.92 \%$ & $6.39 \%$ & $7.75 \%$ & $6.05 \%$ & $10.9 \%$ & $12.27 \%$ \\
\hline (HPDW) & RootGap & $4.75 \%$ & $3.52 \%$ & $2.98 \%$ & $7.72 \%$ & $9.57 \%$ & $10.68 \%$ & $7.94 \%$ & $13.94 \%$ & $15.98 \%$ \\
\hline +SSPI & RootGap & $2.33 \%$ & $1.86 \%$ & $1.39 \%$ & $4.21 \%$ & $6.17 \%$ & $7.67 \%$ & $4.52 \%$ & $10.85 \%$ & $12.57 \%$ \\
\hline \multirow[t]{3}{*}{ (HPL) } & Time & 2.38 & 30.94 & 94.70 & 1760.43 & - & - & $13817^{* 6}$ & - & - \\
\hline & Nodes & 502 & 2111 & 2442 & 283896 & - & - & 1155594 & - & - \\
\hline & Time OptSol & 2.14 & 24.92 & 69.98 & 385.31 & - & - & 4581.85 & - & - \\
\hline NotSolved & FinalGap & - & - & - & - & $5.90 \%$ & $8.84 \%$ & $3.89 \%$ & $13.68 \%$ & $15.23 \%$ \\
\hline (HPDW) & Time & $139.39^{* 1}$ & $2479.69^{* 5}$ & $4792.28^{* 4}$ & - & - & - & - & - & - \\
\hline \multirow[t]{4}{*}{ "Try" } & Nodes & 1445 & 2648 & 3180 & - & - & - & - & - & - \\
\hline & Cols & 8670 & 25366 & 28724 & - & - & - & - & - & - \\
\hline & Iters & 3116 & 5281 & 6111 & - & - & - & - & - & - \\
\hline & TimeOptSol & 12.94 & 715.39 & 238.86 & - & - & - & - & - & - \\
\hline (SP) & Time & 7.44 & 46.17 & 107.07 & - & - & - & - & - & - \\
\hline NotSolved & FinalGap & - & - & - & - & $18.83 \%$ & $25.74 \%$ & $8.80 \%$ & $33.30 \%$ & $34.28 \%$ \\
\hline (HPDW) & Time & 10.69 & 99.61 & 459.17 & - & $16058^{* 9}$ & - & - & - & - \\
\hline \multirow[t]{4}{*}{ “Tun1” } & Nodes & 1841 & 6629 & 14406 & - & 21077 & - & - & - & - \\
\hline & Cols & 1202 & 1515 & 1937 & - & 36750 & - & - & - & - \\
\hline & Iters & 2287 & 6974 & 14709 & - & 37686 & - & - & - & - \\
\hline & TimeOptSol & 2.85 & 26.56 & 212.38 & - & 544.66 & - & - & - & - \\
\hline (SP) & Time & 2.06 & 38.61 & 207.70 & - & 601 & - & - & - & - \\
\hline NotSolved & FinalGap & - & - & - & - & $2.20 \%$ & $17.78 \%$ & $7.59 \%$ & $23.85 \%$ & $28.12 \%$ \\
\hline SolByHeur & Instances & 4 & 5 & 3 & - & 0 & - & - & - & - \\
\hline BestSolVal & Tun1 > Try & 0 & 4 & 4 & 6 & 6 & 7 & 8 & 9 & 8 \\
\hline (HPDW) & Time & 9.04 & 114.34 & 615.03 & - & $15448^{* 9}$ & - & - & - & - \\
\hline \multirow[t]{4}{*}{ “Tun2” } & Nodes & 1750 & 7463 & 19287 & - & 23378 & - & - & - & - \\
\hline & Cols & 1161 & 1525 & 1866 & - & 33211 & - & - & - & - \\
\hline & Iters & 2184 & 7832 & 19591 & - & 39489 & - & - & - & - \\
\hline & TimeOptSol & 1.20 & 20.86 & 172.37 & - & 0.01 & - & - & - & - \\
\hline (SP) & Time & 2.02 & 45.93 & 288.72 & - & 548 & - & - & - & - \\
\hline NotSolved & FinalGap & - & - & - & - & $2.50 \%$ & $17.84 \%$ & $8.43 \%$ & $23.37 \%$ & $27.57 \%$ \\
\hline SolByHeur & Instances & 7 & 6 & 5 & - & 1 & - & - & - & - \\
\hline BestSolVal & Tun2 > Try & 0 & 4 & 4 & 6 & 6 & 9 & 6 & 10 & 8 \\
\hline BestSolVal & Tun2 $>$ Tun1 & - & - & - & 7 & 2 & 5 & 3 & 5 & 4 \\
\hline (HPL) & Time & 5.65 & 41.07 & 147.42 & $894.54^{* 2}$ & - & - & $10003^{* 8}$ & - & - \\
\hline \multirow[t]{2}{*}{ +SSPI } & Nodes & 357 & 1207 & 1783 & 18215 & - & - & 78776 & - & - \\
\hline & Time OptSol & 4.26 & 34.90 & 92.66 & 180.28 & - & - & 2204 & - & - \\
\hline NotSolved & FinalGap & - & - & - & $0.97 \%$ & $6.46 \%$ & $8.34 \%$ & $3.14 \%$ & $12.80 \%$ & $13.12 \%$ \\
\hline (HPDW) & Time & 17.78 & 163.40 & 508.79 & - & - & - & - & - & - \\
\hline “Tun1” & Nodes & 970 & 3235 & 5309 & - & - & - & - & - & - \\
\hline \multirow[t]{3}{*}{ +SSPI } & Cols & 1093 & 1385 & 1710 & - & - & - & - & - & - \\
\hline & Iters & 1343 & 3535 & 5564 & - & - & - & - & - & - \\
\hline & TimeOptSol & 4.85 & 42.89 & 326.65 & - & - & - & - & - & - \\
\hline (SP) & Time & 11.54 & 130.54 & 420.46 & - & - & - & - & - & - \\
\hline NotSolved & FinalGap & - & - & - & - & $12.53 \%$ & $22.36 \%$ & $6.29 \%$ & $26.85 \%$ & $35.05 \%$ \\
\hline SolByHeur & Instances & 4 & 4 & 3 & - & - & - & - & - & - \\
\hline
\end{tabular}

Table 5.3: Numerical results on solving (HP) for complete graph instances, comparing (HPL) and (HPDW) with different configurations of HP-B\&C\&P 


\begin{tabular}{|c|c|c|c|c|c|c|c|c|c|c|}
\hline \multirow{2}{*}{$\begin{array}{l}\text { A1 } \\
\text { instances }\end{array}$} & & \multicolumn{3}{|c|}{21 commodities } & \multicolumn{3}{|c|}{55 commodities } & \multicolumn{3}{|c|}{91 commodities } \\
\hline & & $21 \mathrm{a}$ & 55 a & $91 \mathrm{a}$ & $21 \mathrm{a}$ & $55 \mathrm{a}$ & $91 \mathrm{a}$ & $21 \mathrm{a}$ & $55 \mathrm{a}$ & $91 \mathrm{a}$ \\
\hline (HPL) & RootGap & $5.30 \%$ & $2.78 \%$ & $1.41 \%$ & $6.36 \%$ & $3.57 \%$ & $1.74 \%$ & $6.26 \%$ & $4.34 \%$ & $3.12 \%$ \\
\hline +SSPI & RootGap & $0.32 \%$ & $0.44 \%$ & $0.21 \%$ & $0.64 \%$ & $0.54 \%$ & $0.22 \%$ & $0.72 \%$ & $0.78 \%$ & $0.57 \%$ \\
\hline (HPDW) & RootGap & $3.80 \%$ & $2.30 \%$ & $1.35 \%$ & $3.14 \%$ & $2.52 \%$ & $1.25 \%$ & $2.41 \%$ & $2.97 \%$ & $2.18 \%$ \\
\hline +SSPI & RootGap & $0.32 \%$ & $0.46 \%$ & $0.21 \%$ & $0.49 \%$ & $0.54 \%$ & $0.23 \%$ & $0.63 \%$ & $0.76 \%$ & $0.55 \%$ \\
\hline \multirow[t]{3}{*}{ (HPL) } & Time & 0.28 & 1.62 & 2.34 & 2.58 & 19.37 & 35.23 & 91.53 & 1276.75 & 2057.63 \\
\hline & Nodes & 35 & 93 & 35 & 217 & 802 & 481 & 10135 & 41297 & 29611 \\
\hline & TimeOptSol & 0.26 & 1.35 & 1.70 & 2.24 & 14.08 & 25.06 & 56.22 & 499.29 & 725.18 \\
\hline (HPDW) & Time & 0.49 & 4.42 & 4.14 & 20.22 & 279.61 & 554.85 & $2601.36^{* 2}$ & $254.68^{* 8}$ & $2044.43^{* 7}$ \\
\hline \multirow[t]{4}{*}{ “Try” } & Nodes & 76 & 158 & 87 & 409 & 1429 & 1280 & 2783 & 787 & 1983 \\
\hline & Cols & 541 & 1141 & 1136 & 2253 & 6621 & 5881 & 15731 & 5159 & 11634 \\
\hline & Iters & 183 & 333 & 185 & 1037 & 2910 & 2561 & 6781 & 1765 & 4040 \\
\hline & TimeOptSol & 0.34 & 2.62 & 2.53 & 5.68 & 74.56 & 116.17 & 107.54 & 123.50 & 563.63 \\
\hline (SP) & Time & 0.26 & 2.21 & 3.07 & 8.02 & 114.64 & 260.54 & 247.96 & 180.14 & 1075.37 \\
\hline (HPDW) & Time & 0.50 & 3.61 & 2.39 & 7.53 & 76.64 & 192.44 & $235.25^{* 2}$ & $1375.39^{* 6}$ & $3304.34^{* 6}$ \\
\hline \multirow[t]{4}{*}{ “Tun1” } & Nodes & 102 & 317 & 84 & 349 & 1428 & 1500 & 3755 & 10298 & 9868 \\
\hline & Cols & 256 & 431 & 511 & 857 & 945 & 1120 & 3447 & 2229 & 2185 \\
\hline & Iters & 158 & 326 & 105 & 655 & 1627 & 1619 & 5543 & 10885 & 10300 \\
\hline & TimeOptSol & 0.33 & 2.09 & 1.13 & 1.17 & 13.31 & 77.47 & 4.57 & 26.53 & 104.23 \\
\hline (SP) & Time & 0.21 & 2.24 & 1.78 & 4.32 & 62.22 & 166.85 & 99.99 & 1141.27 & 2900.93 \\
\hline SolByHeur & Instances & 6 & 4 & 6 & 8 & 5 & 7 & 6 & 1 & 3 \\
\hline \multicolumn{11}{|l|}{$\mathrm{DW}<\mathrm{L}:$} \\
\hline Time & Instances & 5 & 8 & 7 & 2 & 1 & 2 & 0 & 0 & 0 \\
\hline TimeOptSol & Instances & 7 & 8 & 8 & 8 & 6 & 7 & 7 & 1 & 2 \\
\hline (HPL) & Time & 0.44 & 20.80 & 5.44 & 6.17 & 42.97 & 54.69 & 424.10 & 795.66 & 1593.86 \\
\hline \multirow[t]{2}{*}{ +SSPI } & Nodes & 14 & 34 & 12 & 64 & 229 & 122 & 1514 & 2119 & 2726 \\
\hline & TimeOptSol & 0.41 & 19.94 & 4.96 & 6.00 & 38.97 & 53.01 & 250.47 & 480.01 & 949.76 \\
\hline (HPDW) & Time & 0.60 & 28.44 & 14.79 & 31.19 & 262.19 & 508.00 & $941.45^{* 2}$ & $7126.76^{* 5}$ & $8546.18^{* 6}$ \\
\hline “Tun1” & Nodes & 11 & 24 & 19 & 51 & 112 & 156 & 479 & 486 & 458 \\
\hline \multirow[t]{3}{*}{ +SSPI } & Cols & 212 & 457 & 518 & 782 & 891 & 1088 & 3774 & 2812 & 2220 \\
\hline & Iters & 56 & 87 & 59 & 282 & 272 & 268 & 1839 & 1229 & 798 \\
\hline & TimeOptSol & 0.45 & 1.24 & 6.84 & 25.79 & 39.85 & 135.51 & 89.44 & 4661.09 & 3502.96 \\
\hline (SP) & Time & 0.47 & 25.31 & 13.84 & 28.23 & 255.27 & 496.84 & 857.95 & 7000.55 & 8445.88 \\
\hline SolByHeur & Instances & 2 & 4 & 5 & 4 & 5 & 5 & 4 & 1 & 3 \\
\hline \multicolumn{11}{|l|}{$\mathrm{DW}<\mathrm{L}:$} \\
\hline Time & Instances & 6 & 2 & 4 & 1 & 0 & 2 & 1 & 0 & 0 \\
\hline TimeOptSol & Instances & 7 & 7 & 6 & 3 & 5 & 7 & 5 & 2 & 5 \\
\hline
\end{tabular}

Table 5.4: Numerical results on solving (HP) for A1 instances, comparing (HPL) and (HPDW) with different configurations of HP-B\&C\&P 


\section{Conclusion}

The bilevel optimisation paradigm provides a rich framework for pricing goods and services, including in network-based problems. Bilevel pricing models are both theoretically and computationally challenging, so it is crucial to exploit the inner structure of each particular case in order to obtain efficient solution methods.

In this thesis we looked at bilevel pricing problems over an underlying network, and used mathematical programming tools to solve them in an efficient way. We achieved this by addressing their computational complexity, introducing new formulations, and proposing different solution methods, both for polynomially solvable and difficult cases.

We introduced two new versions of the network pricing problem with a novel tolling scheme, in which the authority either sets the same toll on all of its toll arcs, or sets a toll proportional to the arcs' lengths. We proved the first case to be polynomial and the latter pseudo-polynomial, and presented a sequential solution algorithm. Furthermore we linked these problems to the parametric shortest path problem, for which results exist in the literature. We then considered an initial robust approach to the network pricing problem, using an interval representation for uncertain parameters. We proposed polynomial algorithms to solve the robust counterpart of some particular cases that were proved to be polynomial in their deterministic versions, therefore maintaining their complexity class. The description of these results is reported in the second part of this thesis, whilst the first part reports a detailed review of the state of the art of bilevel pricing problems.

In the third part of the thesis, we addressed an $\mathcal{N} \mathcal{P}$-hard network pricing problem, where toll arcs are connected such that they constitute a path, as occurs for highways. We proposed a Dantzig-Wolfe reformulation for this problem, in which the resulting subproblem is non linear, but we showed that it is easy to solve, by separating it for each toll path and exploiting the fact that the single toll path case is polynomially solvable. We used these properties to develop another formulation for this problem, which is an extended mixed integer linear formulation, with a very large - but polynomial - number of variables and constraints. We proved that the linear relaxation optimal solutions of this extended formulation and of the Dantzig-Wolfe reformulation coincide, and that they are at least as good as the linear relaxation optimal solution of the mixed-integer linear formulation that was proposed in the literature for this problem. 
Moreover, numerical results for different types of instances showed that the improvement is significant.

The Dantzig-Wolfe reformulation is solved with a column generation algorithm, implemented in SCIP. We proposed several strategies to improve the performance of the algorithm, such as using different initialisations, as well as applying several stabilisation techniques using a dual variable smoothing approach, among others. Numerical results for different types of instances showed that these strategies are very effective in reducing the solution time and also in improving the convergence behaviour of the algorithm, for instance by reducing the dual oscillations and the tailing-off effect. On the more realistic class of instances that we used in our tests, the linear relaxation of the Dantzig-Wolfe reformulation is almost always solved more quickly with the improved column generation algorithm than the linear relaxation of the mixed-integer linear formulation from the literature.

We then addressed the inverse problem of the network pricing problem with connected toll arcs, where we want to find the set of tolls that maximises the authority's revenue, fulfilling a given assignment of commodities to toll paths. Using the similarities between the network pricing problem with connected toll arcs and the product pricing problem with reservation prices, we started from results published in the literature for this latter problem to show that the inverse problem is polynomial and to detail its feasibility conditions. Furthermore we exploited these results to propose heuristic algorithms to construct integer feasible solutions for the problem.

Finally all of these elements were combined, together with the reformulation of some valid inequalities from the literature, into a Branch-and-Cut-and-Price algorithm for the network pricing problem with connected toll arcs. We proposed different branching strategies to construct the tree, using pseudo-cost values and special order sets, and we showed how to include heuristic solutions in this framework. Both the column generation and the Branch-and-Cut-and-Price algorithms where tuned with an automatic tuner of parameters (irace), to find the best performing configurations.There was a large difference in the performance of the algorithms depending on the configuration chosen, and given the large number of parameters to be set, using an automatic tuner was very useful.

Numerical results showed that the configurations found by the tuner significantly outperformed the basic configurations as well as the configurations we initially thought would be good. Despite the promising better root node gap and the improvements to the column generation and Branch-and-Cut-and-Price algorithms, the overall performance of this framework in solving the problem was shown to be worse than the mixed-integer formulation that was proposed in the literature. However, on the more realistic class of instances, the Branch-and-Price algorithm often finds the optimal solution more quickly than the mixed-integer formulation, though it is then slower to prove the optimality of the solution. Numerical results also showed that the heuristic algorithms perform well and often find the optimal solution. 
Given the complexity of the problems addressed, there remains a whole range of interesting and open questions. For instance, one could apply the unit toll approach introduced in this thesis to real problems, and compare the gain in the solution time and the loss in the leader's revenue with respect to the general approach of different tolls. This unit approach could be simpler from a managerial point of view, so if the loss in revenue is not very large, a company may well prefer using it. From a more theoretical point of view, the complexity of the proportional version of the unit toll problem is also still an open question which could be addressed.

We proposed a first robust approach to some simple cases of the network pricing problem; it would certainly be interesting to extend this study, by including different uncertainty representations and/or by solving more cases. Since uncertainty is present in almost all real world problems, it is very important for models to be able to take it into consideration. The difficulty of these problems already in their nominal version will require ad hoc solution methods to solve their robust counterparts.

We presented a Dantzig-Wolfe reformulation and a Branch-and-Cut-and-Price algorithm for the network pricing problem with connected toll arcs: one could also apply the same approach to other pricing problems. Ultimately the results showed that this approach was not very effective for the problem we analysed, and therefore we are not convinced that this is a promising path to follow, unless some improvements are found or other problems reveal other properties which make the algorithms more efficient. Another approach could be to develop cutting planes from the Dantzig-Wolfe and the extended formulations we proposed to tighten the linear relaxation of the mixed-integer formulation from the literature and therefore improve its computational performance.

More generally, it would be interesting to study how to integrate real-life features into bilevel pricing models (e.g. congestion, market segmentation, dynamics, randomness) or how to extend these models to tackle variants of product pricing (e.g. pricing together bundles of different products rather than single units).

Furthermore, one could extend the pricing framework to have multiple authorities or companies competing in a market where users have the choice to buy from any of them. This problem has been studied in economics and game theory, for instance in the "Hotelling problem" (Hotelling, 1929). In this problem a duopoly is considered: two companies sell the same product and customers have different transportation costs which depends on the distance from the company to the customer. Each company chooses its best location knowing the location of the other company as well as the customers. It may be interesting to study different variants of pricing problems with multiple authorities, analysing which conditions can lead to the existence of Nash equilibria (solutions where no participants have an interest in changing their strategy) or other types of equilibria. This approach would have applications in many real problems, such as product pricing situations as well as tolling schemes in air traffic management and other network-related pricing problems. 


\section{Bibliography}

A1data. Profile of a1 highway Milano - Napoli. http://www.automap.it/ autostrade/mappa.asp?tratta=A1. Accessed: 07-08-2014.

T. Achterberg. SCIP: Solving constraint integer programs. Mathematical Programming Computation, 1(1):1-41, 2009. http://mpc.zib.de/index.php/MPC/article/view/4.

R. K. Ahuja, T. L. Magnanti, and J. B. Orlin. Network flows: theory, algorithms, and applications. Prentice hall, 1993.

E. Amaldi, M. Bruglieri, and B. Fortz. On the hazmat transport network design problem. In J. Pahl, T. Reiners, and S. Voß, editors, Network Optimization, volume 6701 of Lecture Notes in Computer Science, pages 327-338. Springer Berlin / Heidelberg, 2011.

I. Averbakh. On the complexity of a class of combinatorial optimization problems with uncertainty. Mathematical Programming, 90(2):263-272, 2001.

C. Barnhart, E. L. Johnson, G. L. Nemhauser, M. W. Savelsbergh, and P. H. Vance. Branch-and-price: Column generation for solving huge integer programs. Operations research, 46(3):316-329, 1998.

E. M. L. Beale and J. A. Tomlin. Special facilities in a general mathematical programming system for non-convex problems using ordered sets of variables. OR, 69(99): $447-454,1970$.

A. Ben-Tal, L. El Ghaoui, and A. Nemirovski. Robust optimization. Princeton University Press, 2009.

M. Benichou, J. Gauthier, P. Girodet, G. Hentges, G. Ribiere, and O. Vincent. Experiments in mixed-integer linear programming. Mathematical Programming, 1(1):76-94, 1971.

D. Bertsimas and M. Sim. Robust discrete optimization and network flows. Mathematical programming, 98(1-3):49-71, 2003.

D. Bertsimas and M. Sim. The price of robustness. Operations research, 52(1):35-53, 2004.

D. Bertsimas, D. B. Brown, and C. Caramanis. Theory and applications of robust optimization. SIAM review, 53(3):464-501, 2011. 
J. R. Birge and F. Louveaux. Introduction to stochastic programming. Springer, 2011.

M. Bouhtou, A. Grigoriev, S. Van Hoesel, A. Van der Kraaij, F. Spieksma, and M. Uetz. Pricing bridges to cross a river. Naval Research Logistics, 54:411-420, 2007a.

M. Bouhtou, S. Van Hoesel, A. Van der Kraaij, and J. Lutton. Tariff optimization in networks. INFORMS Journal on Computing, 19(3):458-469, 2007b.

J. Bracken and J. McGill. Mathematical programs with optimization problems in the constraints. Operations Research, 21(1):37-44, 1973.

L. Brotcorne, M. Labbé, P. Marcotte, and G. Savard. A bilevel model and solution algorithm for a freight tariff-setting problem. Transportation Science, 34(3):289-302, 2000.

L. Brotcorne, M. Labbé, P. Marcotte, and G. Savard. A bilevel model for toll optimization on a multicommodity transportation network. Transportation Science, 35(4):345-358, 2001.

L. Brotcorne, M. Labbé, P. Marcotte, and G. Savard. Joint design and pricing on a network. Operations Research, 56(5):1104-1115, 2008.

W. Candler and R. Norton. Multilevel programming. Technical Report 20, World Bank Development Research Center, Washington DC, USA, 1977.

J. Cardinal, E. Demaine, S. Fiorini, G. Joret, S. Langerman, I. Newman, and O. Weimann. The Stackelberg minimum spanning tree game. Algorithmica, 59:129-144, 2011.

P. J. Carstensen. The complexity of some problems in parametric linear and combinatorial programming. PhD thesis, 1983.

L. Castelli, M. Labbé, and A. Violin. A network pricing formulation for the revenue maximization of european air navigation service providers. Transportation Research Part C: Emerging Technologies, 33(0):214 - 226, 2013.

B. Colson, P. Marcotte, and G. Savard. Bilevel programming: A survey. 4OR: A Quarterly Journal of Operations Research, 3:87-105, 2005.

B. Colson, P. Marcotte, and G. Savard. An overview of bilevel optimization. Annals of Operations Research, 153(1):235-256, 2007.

G. B. Dantzig and P. Wolfe. Decomposition principle for linear programs. Operations Research, 8(1):101-111, 1960.

G. B. Dantzig. Linear programming under uncertainty. Management science, 1(3-4):197206, 1955.

S. Dempe. Foundations of bilevel programming, volume 61 of Nonconvex optimization and its applications. Kluwer Academic Publishers, 2002. 
J. Desrosiers and M. E. Lübbecke. A primer in column generation. Springer, 2005.

S. Dewez. On the toll setting problem. PhD thesis, Université Libre de Bruxelles, 2004.

S. Dewez, M. Labbé, P. Marcotte, and G. Savard. New formulations and valid inequalities for a bilevel pricing problem. Operation Research Letters, 36(2):141-149, 2008.

O. du Merle, D. Villeneuve, J. Desrosiers, and P. Hansen. Stabilized column generation. Discrete Mathematics, 194(1 - 3):229 - 237, 1999.

J. Erickson. Maximum flows and parametric shortest paths in planar graphs. In Proceedings of the Twenty-First Annual ACM-SIAM Symposium on Discrete Algorithms, pages 794-804. Society for Industrial and Applied Mathematics, 2010.

D. Feillet. A tutorial on column generation and branch-and-price for vehicle routing problems. 4-OR - Quarterly Journal of Operations Research, 8:407-424, 2010.

M. Garey and D. Johnson. Computers and Interactability. W.H. Freeman, San Francisco, USA, 1979.

A. Geoffrion. Lagrangean relaxation for integer programming. Approaches to Integer Programming, pages 82-114, 1974.

D. M. Gusfield. Sensitivity analysis for combinatorial optimization. PhD thesis, University of California, Berkeley, 1980.

P. Hansen. Bicriterion path problems. In G. Fandel and T. Gal, editors, Multiple Criteria Decision Making, Theory and Application, Proceedings of the 3rd International Conference, Hagen, Konigswinter 1979, volume 177 of Lecture Notes in Economics and Mathematical Systems, pages 109-127, Berlin, 1980. Springer - Verlag.

P. Hansen, B. Jaumard, and G. Savard. A new branch-and-bound rules for linear bilevel programming. SIAM Journal on Scientific and Statistical Computing, 5(13):1194-1217, 1992.

G. Heilporn, M. Labbé, P. Marcotte, and G. Savard. A parallel between two classes of pricing problems in transportation and marketing. Journal of Revenue and Pricing Management, 9(1/2):110-125, 2010a.

G. Heilporn, M. Labbé, P. Marcotte, and G. Savard. A polyhedral study of the network pricing problem with connected toll arcs. Networks, 3(55):234-246, $2010 \mathrm{~b}$.

G. Heilporn, M. Labbé, P. Marcotte, and G. Savard. Valid inequalities and branch-andcut for the clique pricing problem. Discrete Optimization, 8(3):393-410, 2011.

H. Hotelling. Stability in competition. Economic Journal, 39:41-57, 1929. 
R. Jeroslow. The polynomial hierarchy and a simple model for competitive analysis. Mathematical Programming, 32:146-164, 1985.

G. Joret. Stackelberg network pricing is hard to approximate. Networks, 57(2):117-120, 2011.

B. Y. Kara and V. Verter. Designing a road network for hazardous materials transportation. Transportation Science, 38(2):188-196, 2004.

R. M. Karp and J. B. Orlin. Parametric shortest path algorithms with an application to cyclic staffing. Discrete Applied Mathematics, 3(1):37-45, 1981.

P. Kouvelis and G. Yu. Robust discrete optimization and its applications, volume 14. Springer, 1997.

M. Labbé, P. Marcotte, and G. Savard. A bilevel model of taxation and its application to optimal highway pricing. Management Science, 44(12):1608-1622, 1998.

M. López-Ibánez, J. Dubois-Lacoste, T. Stützle, and M. Birattari. The irace package, iterated race for automatic algorithm configuration. IRIDIA, Université Libre de Bruxelles, Belgium, Tech. Rep. TR/IRIDIA/2011-004, 2011.

P. Loridan and J. Morgan. Weak via strong Stackelberg problem: New results. Journal of Global Optimization, 8:263-287, 1996.

M. E. Lübbecke and J. Desrosiers. Selected topics in column generation. Operations Research, 53(6):1007-1023, 2005.

P. Marcotte, A. Mercier, G. Savard, and V. Verter. Toll policies for mitigating hazardous materials transport risk. Transportation Science, 43(2):228-243, 2009.

R. E. Marsten. The use of the boxstep method in discrete optimization. Springer, 1975.

R. E. Marsten, W. W. Hogan, and J. W. Blankenship. The boxstep method for large-scale optimization. Operations Research, 23(3):389-405, 1975.

A. Migdalas. Bilevel programming in traffic planning: Models, methods and challenge. Journal of Global Optimization, 7:381-405, 1995.

K. Mulmuley and P. Shah. A lower bound for the shortest path problem. In Computational Complexity, 2000. Proceedings. 15th Annual IEEE Conference on, pages 14-21. IEEE, 2000.

G. Owen. Game Theory. Emerald Group Publishing Limited, 1968.

A. Pessoa, E. Uchoa, M. P. Aragão, and R. Rodrigues. Exact algorithm over an arctime-indexed formulation for parallel machine scheduling problems. Mathematical Programming Computation, 2:259-290, 2010. 
A. Pessoa, R. Sadykov, E. Uchoa, and F. Vanderbeck. In-out separation and column generation stabilization by dual price smoothing. In Experimental Algorithms, pages 354-365. Springer, 2013.

S. Roch, P. Marcotte, and G. Savard. Design and analysis of an approximation algorithm for stackelberg network pricing. Networks, 46(1):57-67, 2005.

M. T. Rosenberg. http://geography.about.com/library/weekly/ aa031601a.htm, Accessed: 07-08-2014.

R. Shioda, L. Tunçel, and T. Myklebust. Maximum utility product pricing models and algorithms based on reservation price. Computational Optimization and Applications, 48:157-198, 2011.

A. L. Soyster. Technical note - convex programming with set-inclusive constraints and applications to inexact linear programming. Operations research, 21(5):1154-1157, 1973.

H. Stackelberg. The Theory of Market Economy. Oxford University Press, 1952.

A. Van Ackere. The principal/agent paradigm: its relevance to various functional fields. European Journal of Operational Research, 70(1):83-103, 1993.

S. Van Hoesel. An overview of Stackelberg pricing in networks. European Journal of Operational Research, 189(3):1393-1402, 2008.

F. Vanderbeck. On Dantzig-Wolfe decomposition in integer programming and ways to perform branching in a branch-and-price algorithm. Operations Research, 48(1): 111-128, 2000.

L. Vicente and P. Calamai. Bilevel and multilevel programming: A bibliography review. Journal of Global Optimization, 5:291-306, 1994.

L. Vicente, G. Savard, and J. Júdice. Descent approaches for quadratic bilevel programming. Journal of Optimization Theory and Applications, 81(2):379-399, 1994.

P. Wentges. Weighted Dantzig-Wolfe decomposition for linear mixed-integer programming. International Transactions in Operational Research, 4(2):151-162, 1997.

L. A. Wolsey. Integer Programming. Series in Discrete Mathematics and Optimization. Wiley-Interscience, 1998.

N. E. Young, R. E. Tarjant, and J. B. Orlin. Faster parametric shortest path and minimum-balance algorithms. Networks, 21(2):205-221, 1991. 
